
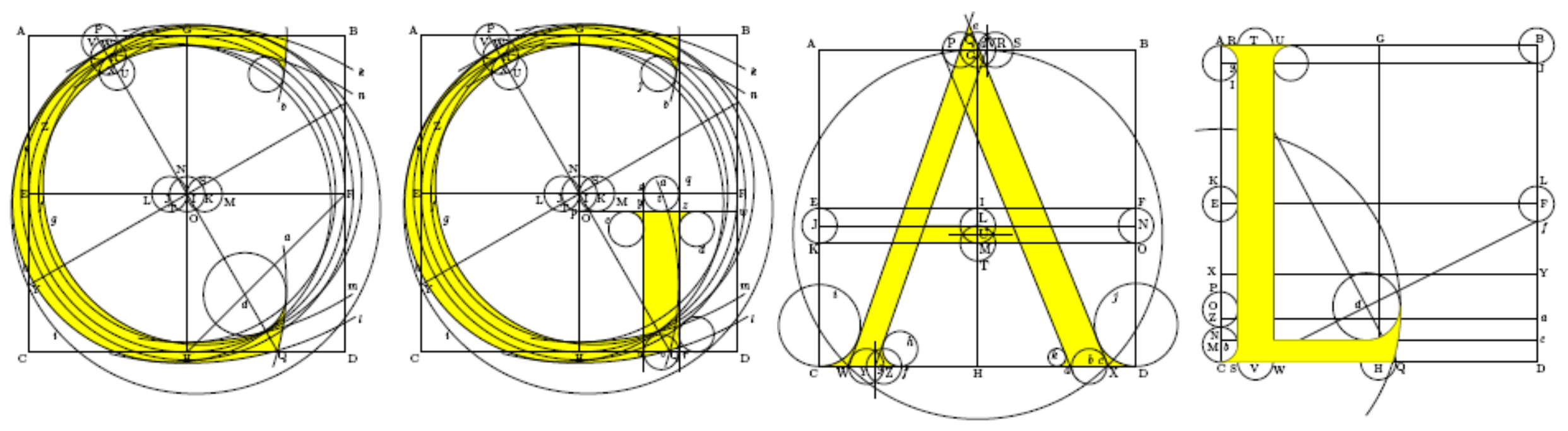

\title{
Computational Geometry Algorithms Library
}

Pierre Alliez INRIA
Andreas Fabri GeometryFactory
Efi Fogel

Tel-Aviv University 


\section{Abstract}

The CGAL Open Source Project provides easy access to efficient and reliable geometric algorithms in the form of a C++ library, offering geometric data structures and algorithms, which are efficient, robust, easy to use, and easy to integrate in existing software. The usage of de facto standard libraries increases productivity, as it allows software developers to focus on the application layer. This course is targeted at software developers with geometric needs, and course graduates will be able to select and use the appropriate algorithms and data structures provided by CGAL in their current or upcoming projects.

\section{Key Facts}

CGAL 3.3, released in June 2007: 90 software components, 600,000 lines of code, 3,500 user and reference manual pages, Cross platform support, Annual release with 12,000 downloads, 1,000 subscribers on the user mailing list, 40 subscribers on the developer mailing list. CGAL is used in many application areas by companies as Total (Oil\&gas), British Telecom (Telecom), Cadence (VLSI), Leica Geosystems (GIS), Dassault Systèmes (CAD), The Moving Picture Company (Visual effects).

\section{CGAL Project}

The project is steered by an Editorial Board, it has a well defined development process, and the infrastructure for distributed development. The following research institutes and companies are actively involved or made contributions to the library: INRIA-Sophia-Antipolis, Max-Planck Institute for Computer Science, Tel-Aviv University, GeometryFactory, ETH Zurich, FU Berlin, University of Groningen, University of Utrecht, Stanford University, Athens University, and the Foundation of Research and Technology - Hellas. For more information on the project see www.cgal.org For an overview on what is in CGAL:

http://www.cgal.org/Manual/3.3/doc html/cgal manual/packages.html 


\section{Organization of the Course}

This course starts with an overview followed by three in-depth sessions covering central data structures: The overview session presents the CGAL project and the design principles of CGAL. CGAL has adopted the exact computing paradigm, which yields robust and at the same time fast algorithms. CGAL further has adopted the generic programming paradigm, which makes CGAL particularly easy to customize and to integrate. Finally, we show how CGAL fits naturally with the STL, and the Boost graph library.

The session on polyhedral surfaces presents the underlying halfedge data structure and how it can be customized to user needs. We further present algorithms for polyhedral surfaces like parameterization, mesh subdivision and simplification, Boolean operations, and intersection detection.

The session on arrangements presents the arrangement API and several data structures built on top of it. These are 3D Minkowski sums, which can be used for collision detection, and 3D lower envelopes, which can be used for visibility map computations.

The last session covers the 2D and 3D triangulation API as well as the surface and volume mesh generators, which are based on Delaunay refinement.

The source code of the examples will be made available at http://www.cgal.org/Courses/SIGGRAPH2008. 


\section{Presenters}

Dr. Andreas Fabri (organizer)

Chief Officer

GeometryFactory

20, Avenue Yves Emmanuel Baudoin 06130 Grasse, FRANCE

Email: andreas.fabri@geometryfactory.com

http://www.geometryfactory.com/

\section{Dr. Efi Fogel}

Senior Researcher

Faculty of Exact Sciences. Tel Aviv University Tel Aviv 69978, ISRAEL

Email: efifogel@gmail.com

http://www.cs.tau.ac.il/ efif/

\section{Dr. Pierre Alliez}

Senior Researcher

INRIA Sophia Antipolis - Mediterranee

2004 route des Lucioles BP 93 - GEOMETRICA, FRANCE

Email: pierre.alliez@sophia.inria.fr

http://www-sop.inria.fr/geometrica/team/Pierre.Alliez/ 


\section{Biographies}

Andreas Fabri, PhD, GeometryFactory As member of the initial development team of the CGAL project, Andreas Fabri is one of the architects of the CGAL software. For several years he chaired the CGAL Editorial Board. In 2003 Andreas founded the GeometryFactory as spin-off of the CGAL project, offering licenses, service and support to commercial users, who cannot comply with the Open Source license of CGAL. Andreas received his PhD in computer science in 2004 from Ecole de Mines de Paris while working on geometric algorithms for parallel machines at INRIA.

Pierre Alliez obtained his PhD from Ecole nationale supérieure des Télécommunications, did his postdoc at Caltech, and is researcher at INRIA since 2001. His main research interests are on topics commonly referred to as Geometry Processing: geometry compression, surface approximation, mesh parameterization, surface remeshing and mesh generation. He is this year co-chair of the EUROGRAPHICS Symposium on Geometry Processing. In 2005 Pierre Alliez received the Eurographics Young Researcher Award.

Efi Fogel, MSc, Tel-Aviv University Efi Fogel is a co-founder of LucidLogix Ltd., a startup company that intends to deliver high performance 3D graphics systems. Efi Fogel is completing his Ph.D. studies at Tel-Aviv University. 3D Graphics and Computational Geometry are his main areas of interests. He is a member of the CGAL Editorial Board, and he is deeply involved with the design and implementation of the arrangement package of CGAL and its derivatives. Efi Fogel received his M.Sc. from Stanford University in 1989. He worked for Silicon Graphics Inc. (SGI) between 19891997 at the Advanced Graphics Devision, where he contributed to the specification of OpenGl among the other. After that Efi worked for Immersia Ltd, and he served as the CTO of Enbaya Ltd. 


\section{Course Syllabus}

Overview

Andreas

$30^{\prime}$

Polyhedron

Pierre

$40^{\prime}$

Arrangements

Efi

$40^{\prime}$

Break

$20^{\prime}$

Triangulations \& Meshes Andreas, Pierre 80'

Wrap-up, Q\&A

All

$15^{\prime}$ 


\section{Bibliography}

- Bibliographic entries for individual chapters of CGAL manuals

- CGAL User and Reference Manual Bibliography 


\section{CGAL Contributors}

\section{CGAL Editorial Board}

Sylvain Pion, Pierre Alliez, Eric Berberich , Andreas Fabri, Efi Fogel, Bernd Gärtner, Michael Hemmer, Michael Hoffmann, Menelaos Karavelas, Marc Pouget, Monique Teillaud, Ron Wein

\section{CGAL Developers}

Fernando Cacciola, Frédéric Cazals, Raphaëlle Chaine, Pavel Emeliyanenko, Marc Glisse, Luc Habert, Peter Hachenberger, Idit Haran, Thomas Herrmann, Samuel Hornus, Michael Kerber, Nico Kruithof, Sebastian Limbach, Sébastien Loriot, Pedro Machado Manhães de Castro, Andreas Meyer, Michal Meyerovitch, Thanh-Trung Nguyen, Luis Peñaranda, Joachim Reichel, Laurent Rineau, Daniel Russel, Laurent Saboret, Ophir Setter, Tel-Aviv University, George Tzoumas

\section{Alumni}

Matthias Bäsken, Hervé Brönnimann, Tran Kai Frank Da, Christophe Delage, Olivier Devillers, Katrin Dobrindt, Eti Ezra, Kaspar Fischer, Eyal Flato, Julia Flötotto, Wolfgang Freiseisen, Geert-Jan Giezeman, Philippe Guigue, Iddo Hanniel, Sariel Har-Peled, Susan Hert, Shai Hirsch, Lutz Kettner, Eran Leiserowitz, Bruno Levy, Eugene Lipovetsky, Abdelkrim Mebarki, Naceur Meskini, Oren Nechushtan, Gabriele Neyer, Steve Oudot, Eli Packer, Dmitrii Pasechnik, Sigal Raab, François

Rebufat, Niv Sabath, Stefan Schirra, Sven Schönherr, Michael Seel, Le-Jeng Shiue, Hans Tangelder, Radu Ursu, Carl Van Geem, Remco Veltkamp, Wieger Wesselink, Afra Zomorodian, Tali Zvi, Baruch Zukerman

\section{EU Project Board Members}

Helmut Alt, Jean-Daniel Boissonnat, Dan Halperin, Kurt Mehlhorn, Stefan Näher, Mark Overmars, Geert Vegter, Emo Welzl, Peter Widmayer 

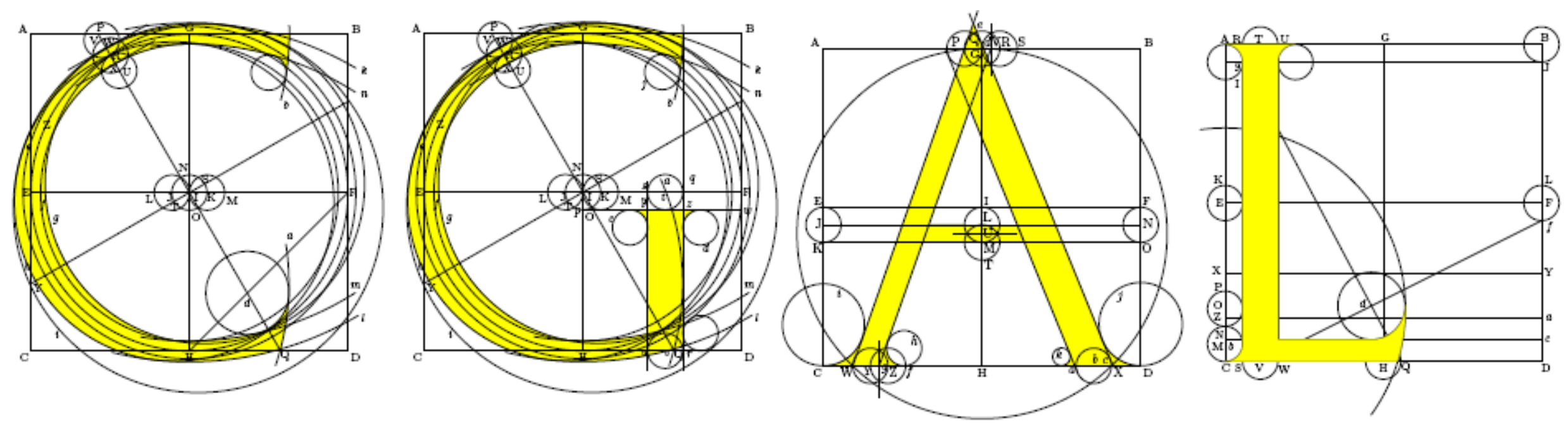

\section{Computational Geometry Algorithms Library}

Pierre Alliez Andreas Fabri INRIA
GeometryFactory
Efi Fogel

Tel Aviv University 


\section{Course Outline}

Overview

Andreas $\quad 30^{\prime}$

Polyhedron

Pierre

$40^{\prime}$

Arrangements

Efi

$40^{\prime}$

Break

$15^{\prime}$

2D Triangulations \& Meshes Andreas 40'

3D Triangulations \& Meshes Pierre 40'

Wrap-up, Q\&A 


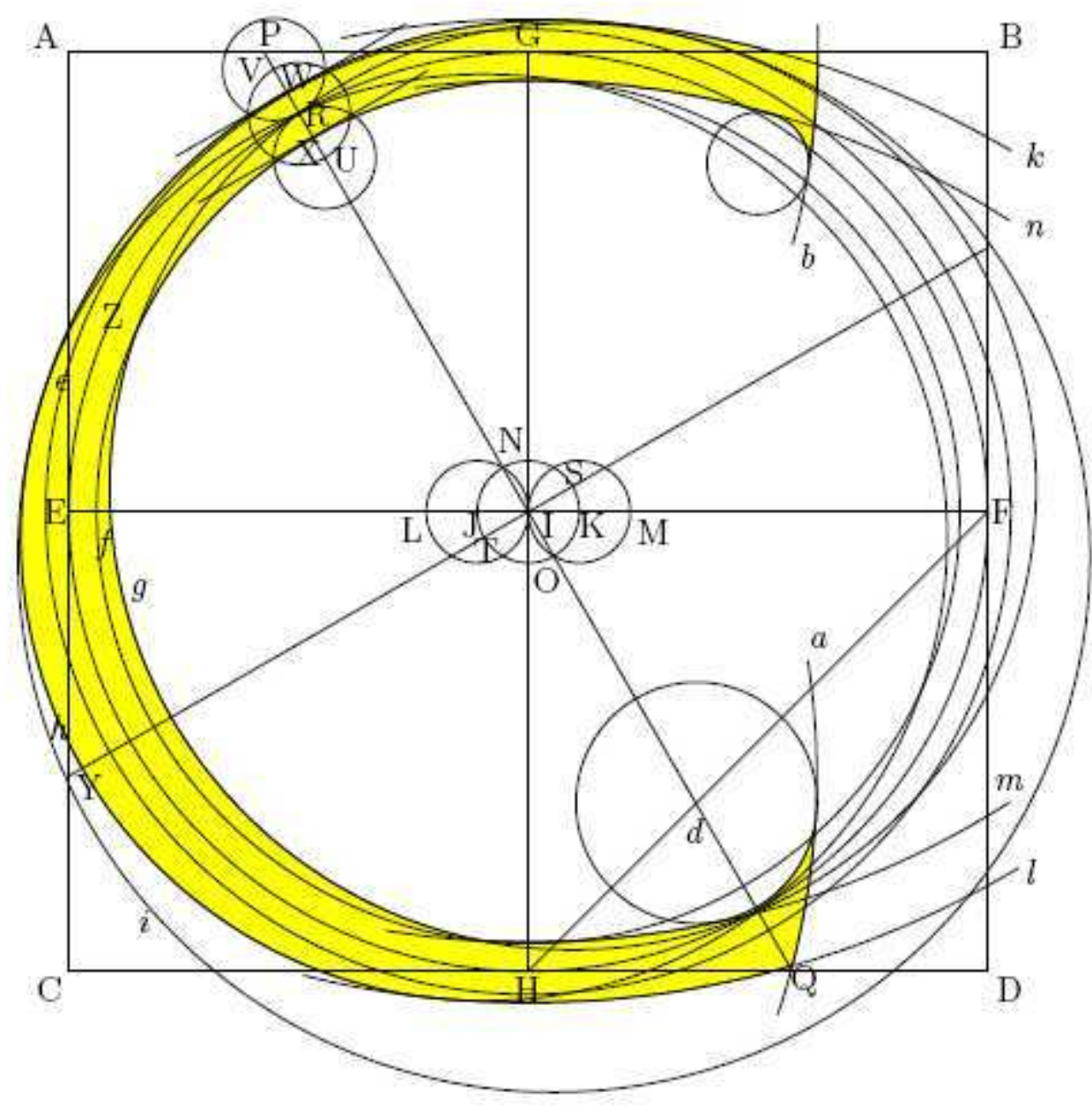

\title{
Overview
}

\author{
Andreas Fabri \\ GeometryFactory
}




\section{Mission Statement}

"Make the large body of geometric algorithms developed in the field of computational geometry available for industrial applications"

CGAL Project Proposal, 1996 


\section{Algorithms and Datastructures}

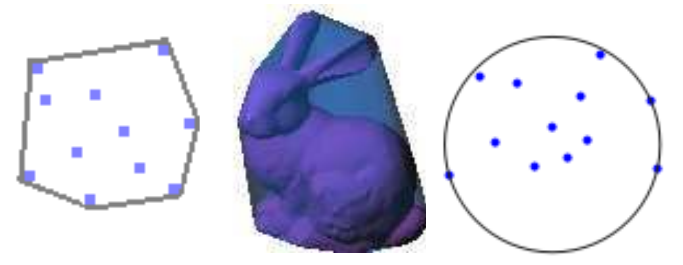

Bounding Volumes

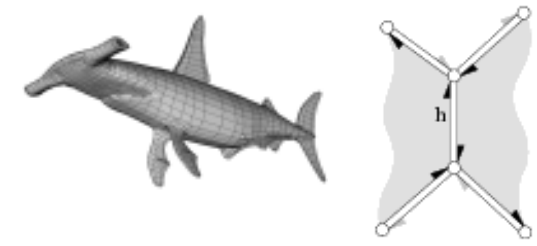

Polyhedral Surface
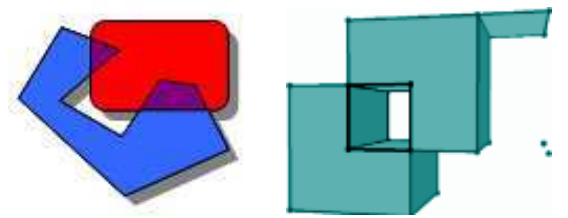

BooleanOperations

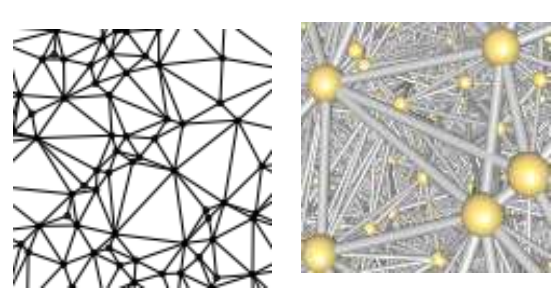

Triangulations
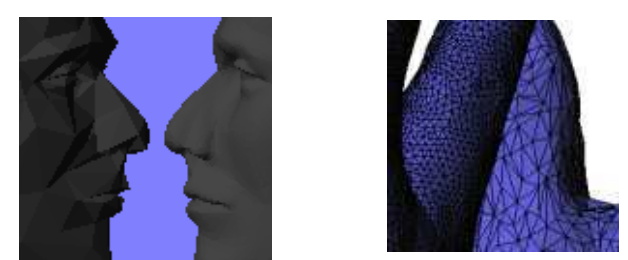

Subdivision Simplification
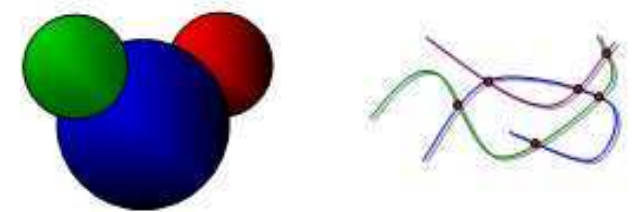

Lower Envelope Arrangement

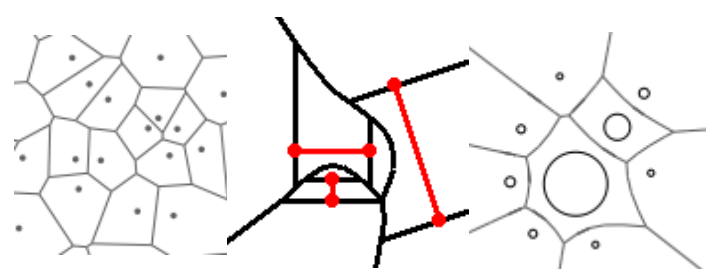

Voronoi Diagrams

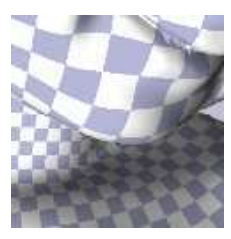

Parametrisation Streamlines
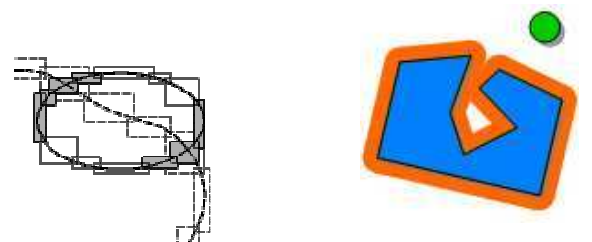

Intersection Minkowski Detection Sum

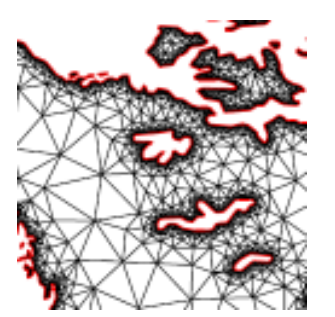

Mesh Generation
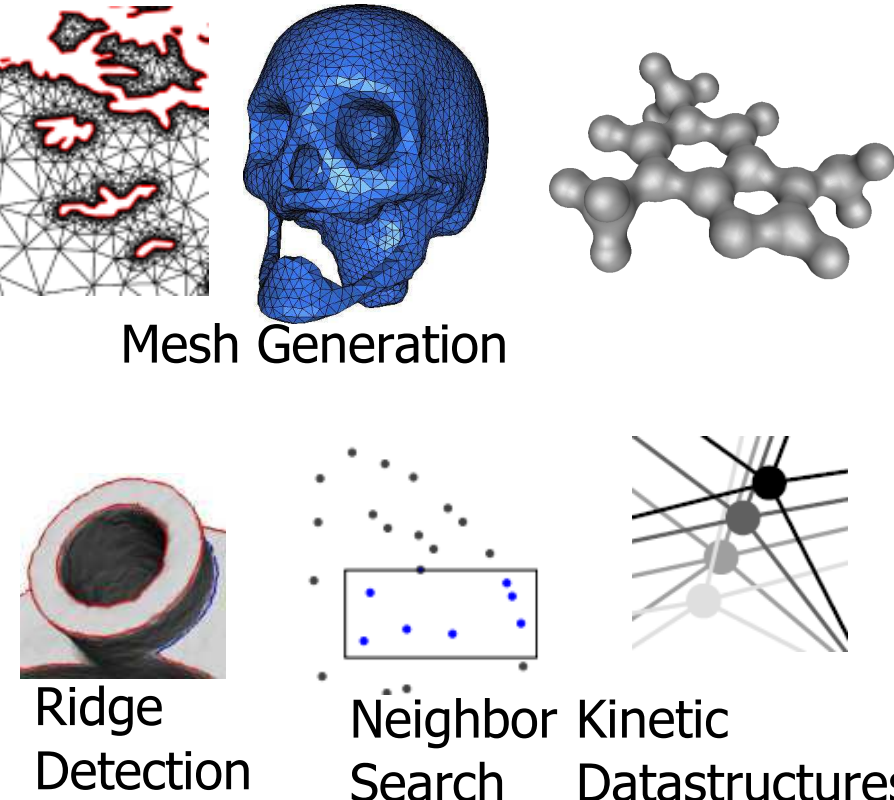

Nëighbor Kinetic Search Datastructures

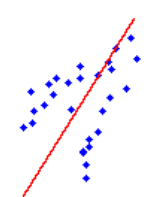

PCA

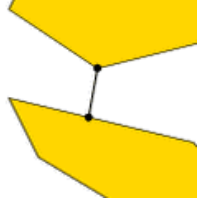

Polytope distance

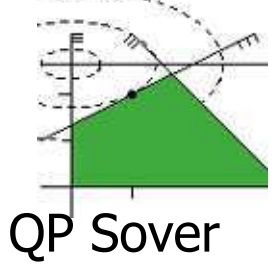




\section{CGAL in Numbers}

500,000 lines of $\mathrm{C}++$ code

10,000 downloads/year (+ Linux distributions)

3,500 manual pages

3,000 subscribers to cgal-announce

1,000 subscribers to cgal-discuss

120 packages

60 commercial users

20 active developers

12 months release cycle

2 licenses: Open Source and commercial 


\section{Some Commercial Users}

cādence" pulsise

Orbotech TOSHIBA

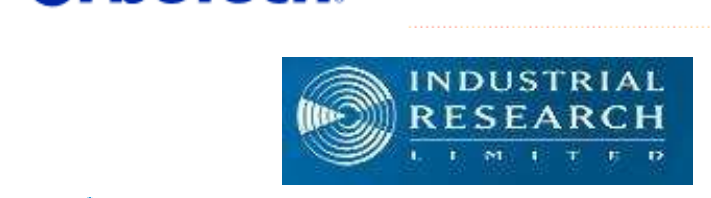

Agilent T
BAE S
Peica
Geossstems

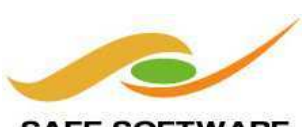

SAFE SOFTWARE
(1) VDRC
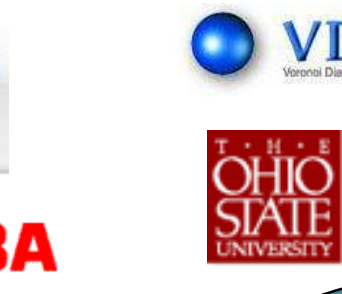

STIE

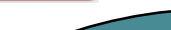

\section{Image} Processing

VLSI
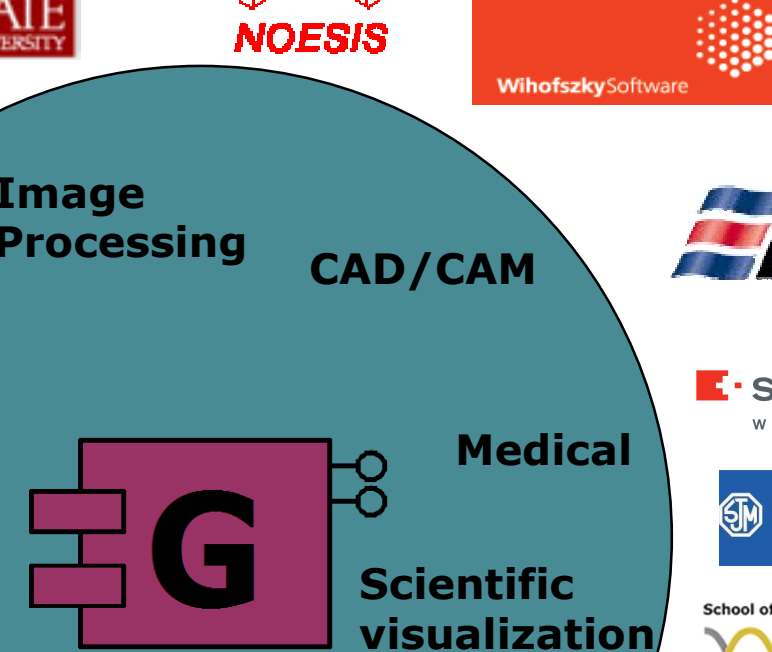

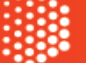

Wihotszky software

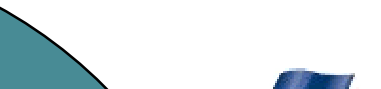

AD /CAM

\section{GECL}

ZWCAD

\section{DS \\ FEMES \\ 7}

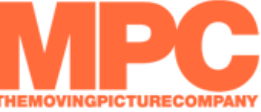

E. schaerermayfield

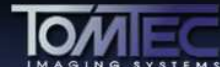

(50.j) ST. JUDE MEDICAL

Scientific visualization

xom

OOOO

NThe MathWorks

Geophysics

(Oil\&Gas)

ifo

Midland Valley

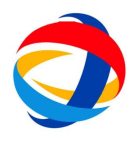

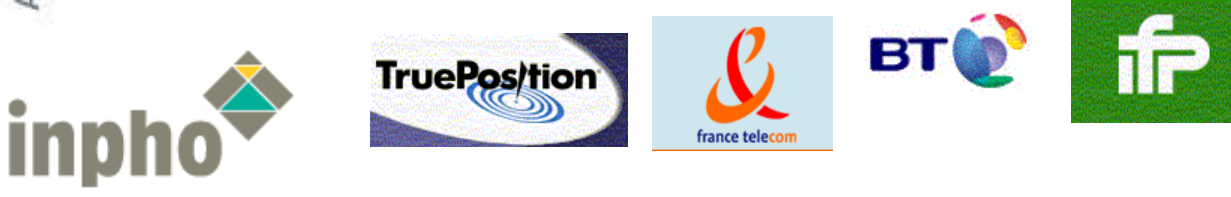




\section{Why They Use CGAL}

" I recommended to the senior management that we start a policy of buying-in as much functionality as possible to reduce the quantity of code that our development team would have to maintain.

This means that we can concentrate on the application layer and concentrate on our own problem domain." 


\section{Why They Use CGAL}

" My research group JYAMITI at the Ohio State University uses CGAL because it provides an efficient and robust code for Delaunay triangulations and other primitive geometric predicates. Delaunay triangulation is the building block for many of the shape related computations that we do. [...]

Without the robust and efficient codes of CGAL, these codes could not have been developed. " 


\section{CGAL Open Source Project}




\section{Project $=\ll$ Planned Undertaking}

- Institutional members make a long term commitment: Inria, MPI, Tel-Aviv U, Utrecht U, Groningen U, ETHZ, GeometryFactory, FU Berlin, Forth, U Athens

- Editorial Board

- Steers and animates the project

- Reviews submissions

- Development Infrastructure

- Gforge: svn, tracker, nightly testsuite,...

- 120p developer manual and mailing list

- Two 1-week developer meetings per year 


\section{Contributions}

- Submission of specifications of new contributions

- Review and decision by the Editorial Board

- Value for contributor

- Integration in the CGAL community

- Gain visibility in a mature project

- Publication value for accepted contributions 


\section{Exact Geometric Computing}




\section{Predicates and Constructions}

Predicates

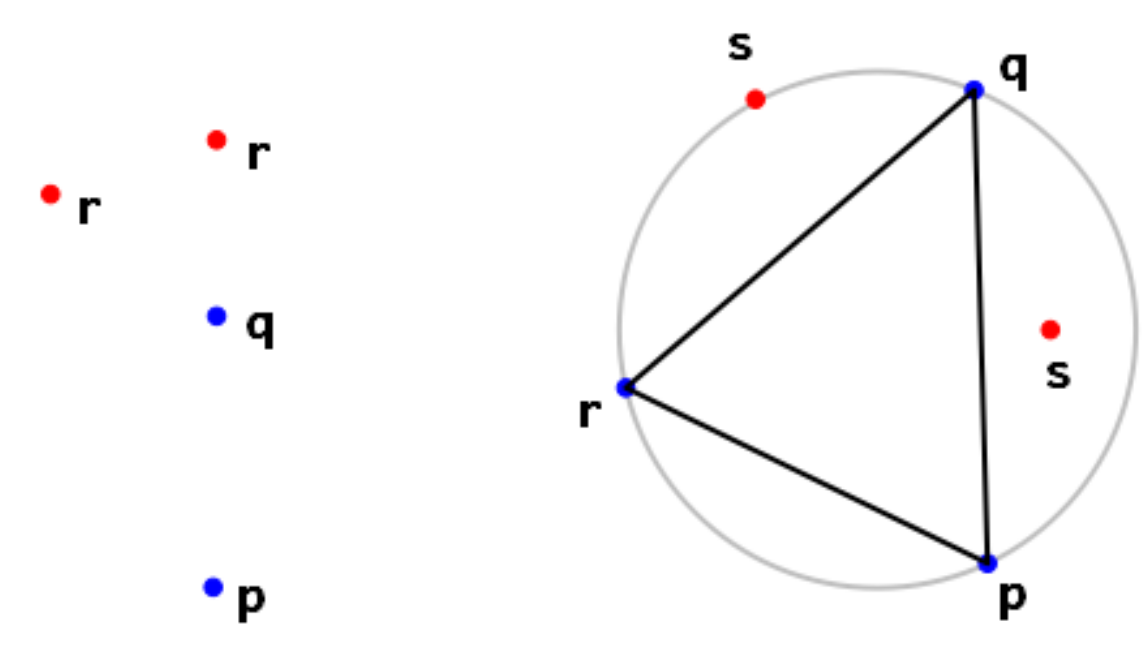

orientation in_circle
Constructions

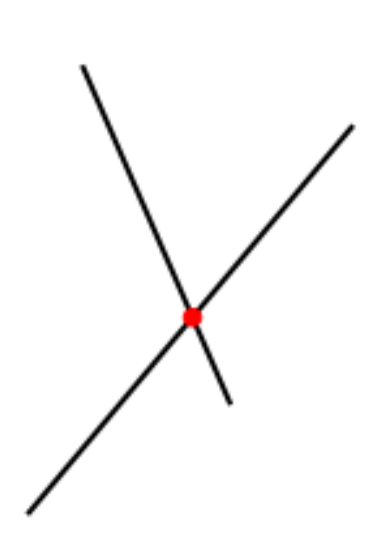

intersection

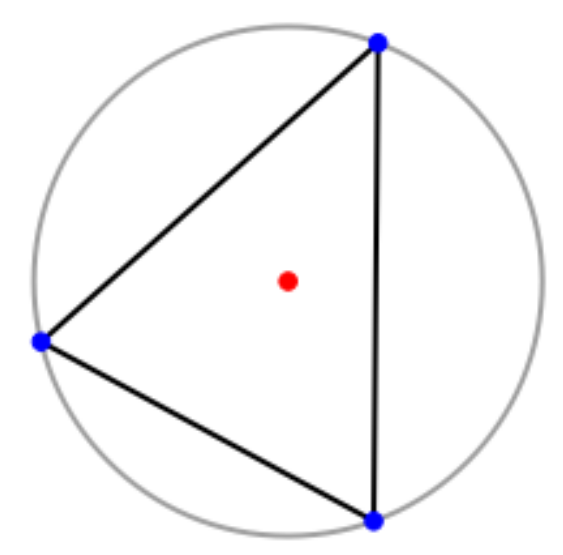

circumcenter 


\section{Robustness Issues}

- Naive use of floating-point arithmetic causes geometric algorithms to:

- Produce [slightly] wrong output

- Crash after invariant violation

- Infinite loop

- There is a gap between

- Geometry in theory

- Geometry with floating-point arithmetic 


\section{Geometry in Theory}

$\operatorname{ccw}(s, q, r) \& \operatorname{ccw}(p, s, r) \& \operatorname{ccw}(p, q, s) \Rightarrow \operatorname{ccw}(p, q, r)$

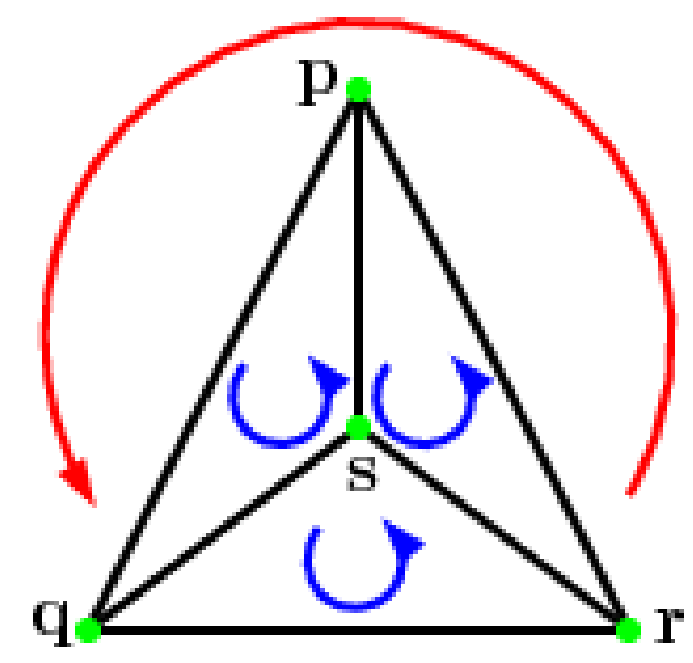

Correctness proofs of algorithms rely on such theorems 


\section{The Trouble with Double}

orientation $\left(p, q_{,}, r\right)=\operatorname{sign}\left(\left(p_{x}-r_{x}\right)\left(q_{y}-r_{y}\right)-\left(p_{y}-r_{y}\right)\left(q_{x}-r_{x}\right)\right)$
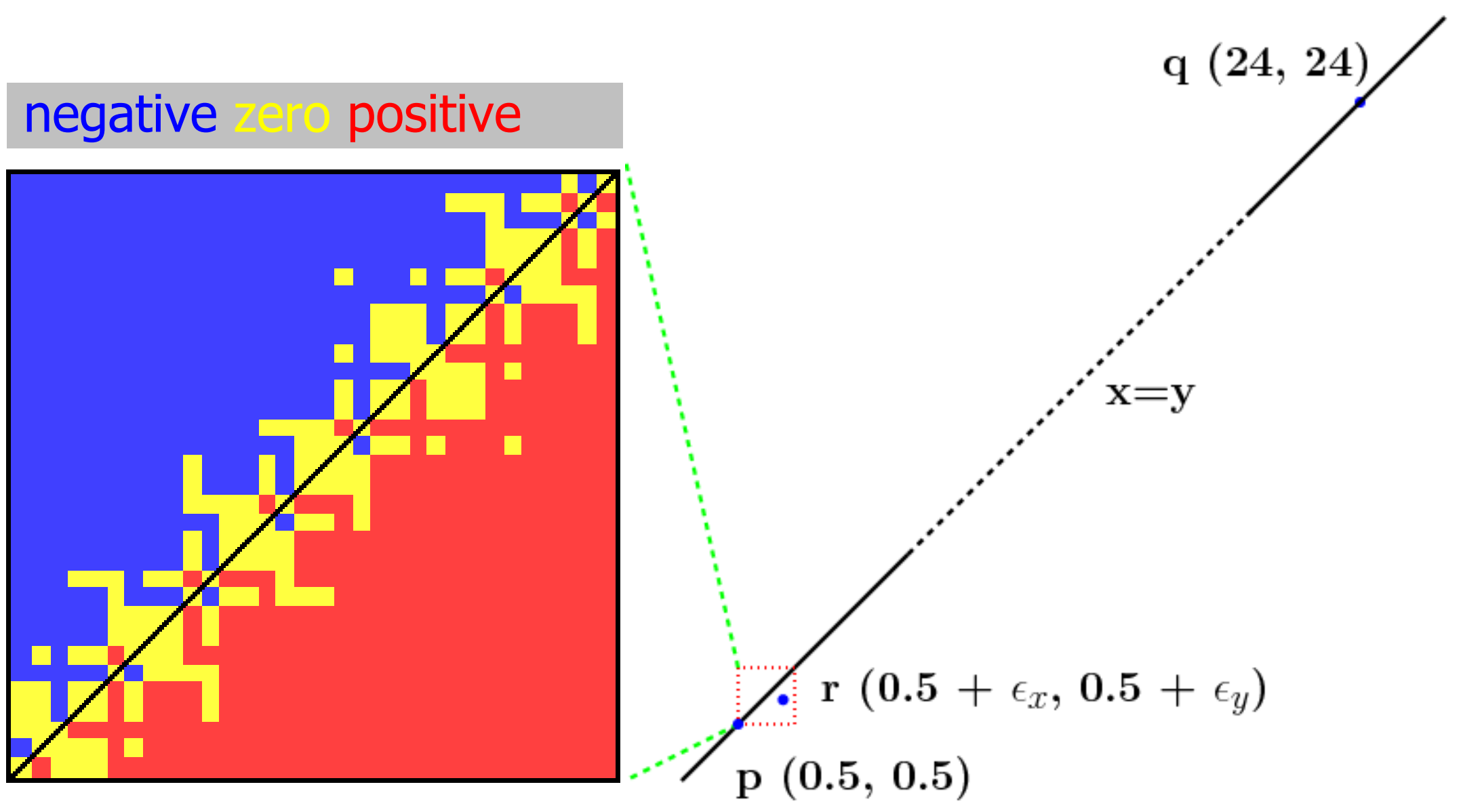


\section{Exact Geometric Computing [Yap]}

Make sure that the control flow in the implementation corresponds to the control flow with exact real arithmetic
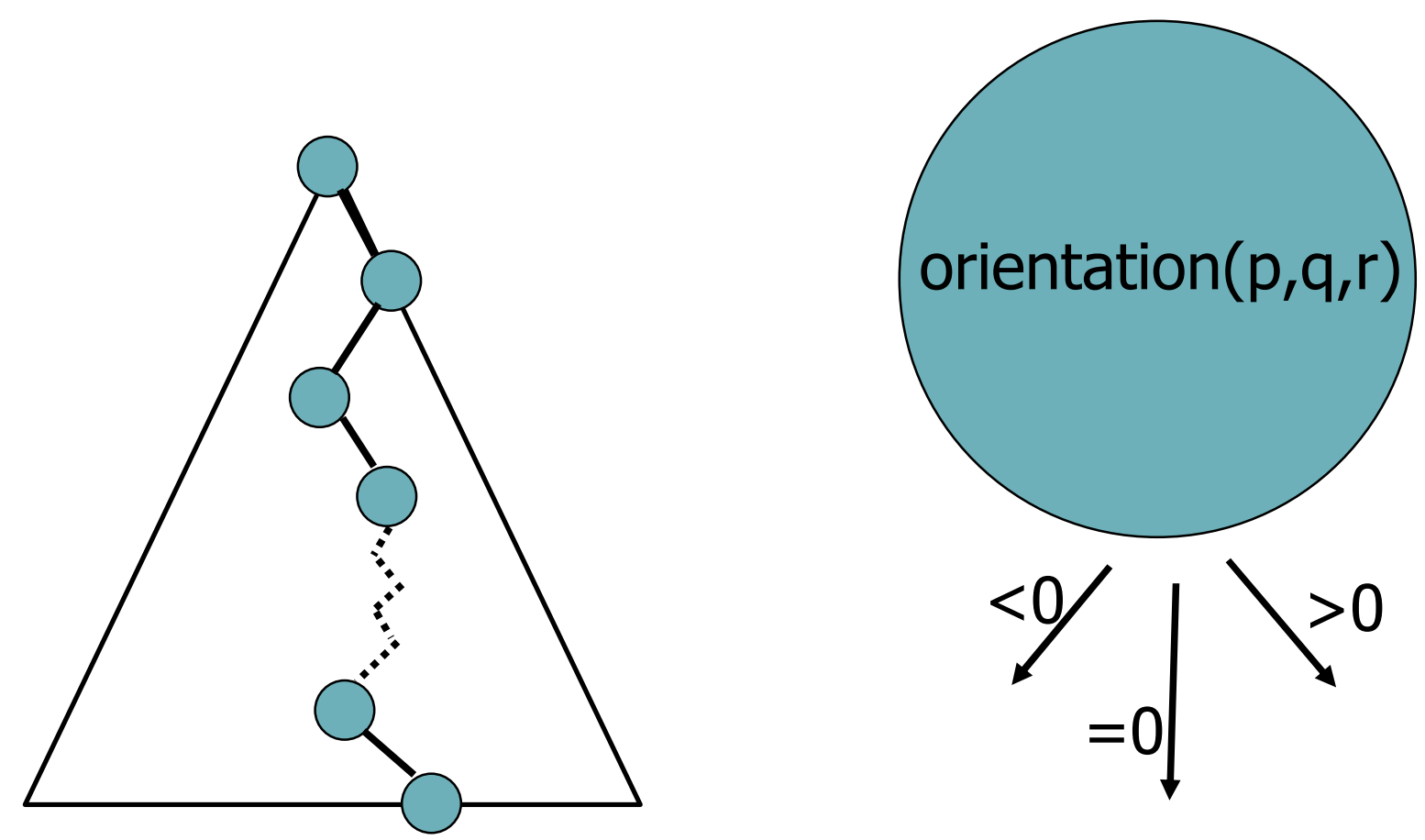


\section{Filtered Predicates}

- Generic functor adaptor Filtered_predicate<>

- Try the predicate instantiated with intervals

- In case of uncertainty, evaluate the predicate with multiple precision arithmetic

- Refinements:

- Static error analysis

- Progressively increase precision 


\section{Filtered Constructions}

Lazy number $=$ interval and arithmetic expression tree

$$
(3.2+1.5) * 13
$$

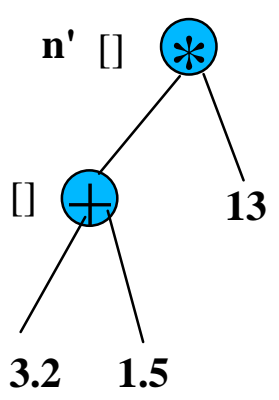

Lazy object $=$ approximated object and geometric operation tree
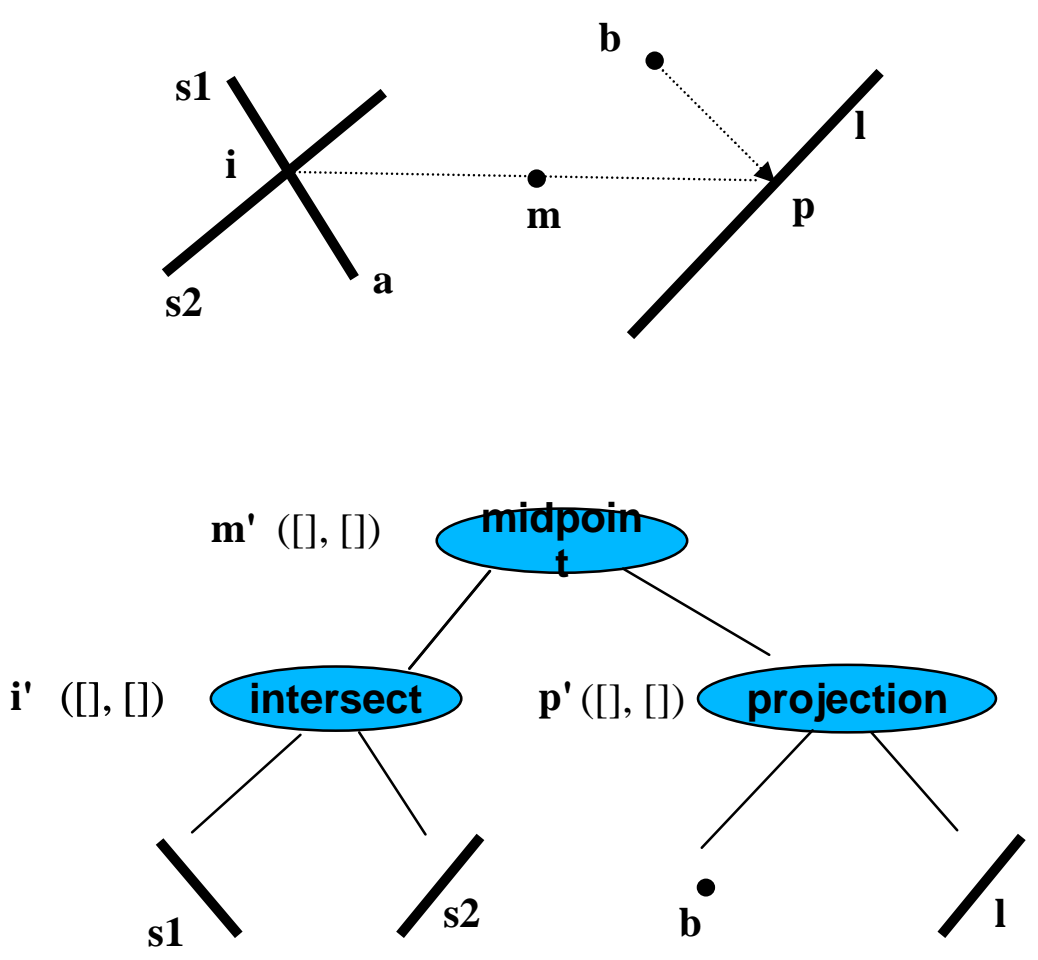

Test that may trigger an exact re-evaluation:

$$
\text { if }\left(n^{\prime}<m^{\prime}\right)
$$$$
\text { if (collinear( } \left.\left(a^{\prime}, m^{\prime}, b^{\prime}\right)\right)
$$ 


\section{The User Perspective}

- Convenience Kernels

- Exact_predicates_inexact_constructions_kernel

- Exact_predicates_exact_constructions_kernel

- Exact_predicates_exact_constructions_kernel_with_sqrt

- Number Types

- double, float

- CGAL::Gmpq (rational), Core (algebraic)

- CGAI : :Lazy_exact_nt<ExactNT>

- Kernels

- CGAI: :Cartesian<NT>

- CGAL : :Filtered_kernel<Kernel>

- CGAL: : Lazy_kernel<NT> 


\section{Merits and Limitations}

- Ultimate robustness inside the black box

- The time penalty is reasonable, e.g. $10 \%$ for 3D Delauny triangulation of $1 \mathrm{M}$ random points

- Limitations of Exact Geometric Computing

- Topology preserving rounding is non-trivial

- Construction depth must be reasonable

- Cannot handle trigonometric functions 


\section{Generic Programming}




\section{STL Genericity}

template <class Key, class Less>

class set \{

Less less;

insert (Key k)

f

if (less (k, treenode.key))

insertLeft (k);

else

insertRight (k);

\}

\};

STL manual 


\section{CGAL Genericity}

template < class Geometry >

class Delaunay_triangulation_2 \{

Geometry: : Orientation orientation;

Geometry: In_circle in_circle;

void insert (Geometry: : Point $t$ ) \{

if (in_circle $(p, q, r, t))\{\ldots\}$

if (orientation $(p, q, r)\{\ldots\}$

\}

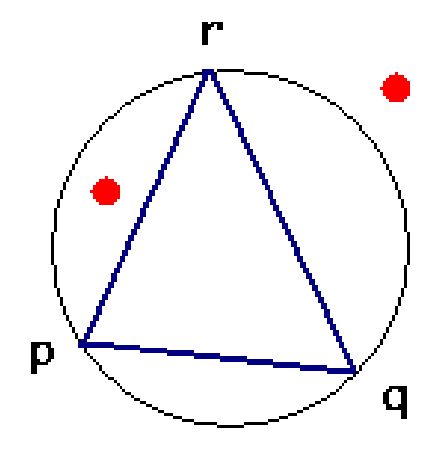

\}; 


\section{CGAL Genericity}

template < class Geometry, class TDS > class Delaunay_triangulation_2 \{

\} ;

template < class Vertex, class Face > class Triangulation_data_structure_2 \{

\} ; 


\section{Iterators}

template <class Geometry>

class Delaunay_triangulation_2 \{

typedef .. Vertex_iterator;

typedef .. Face_iterator;

Vertex_iterator vertices_begin();

Vertex_iterator vertices_end();

template <class OutputIterator>

incident_faces (Vertex_handle v, OutputIterator it);

\};

std: : list<Face_handle> faces;

$d t$.incident_faces ( $v$, std: :back_inserter(faces)) ; 


\section{Iterators}

template <class Geometry>

class Delaunay_triangulation_2 \{

template <class $T>$

void insert ( $T$ begin, $T$ end) $; / /$ typeof (*begin)==Point \} ;

list<Kernel::Point_2> points;

Delaunay_triangulation<Kernel> dt;

dt.insert (points.begin(), points.end()); 


\section{Boost Graph Library (BGL)}

- Rich collection of graph algorithms: shortest paths, minimum spanning tree, flow, etc.

- Design that

- decouples data structure from algorithm

- links them through a thin glue layer

- BGL and CGAL

- Provide glue layer for CGAL

- Extension to embedded graphs inducing the notion of faces 


\section{BGL Glue Layer: Traits Class}

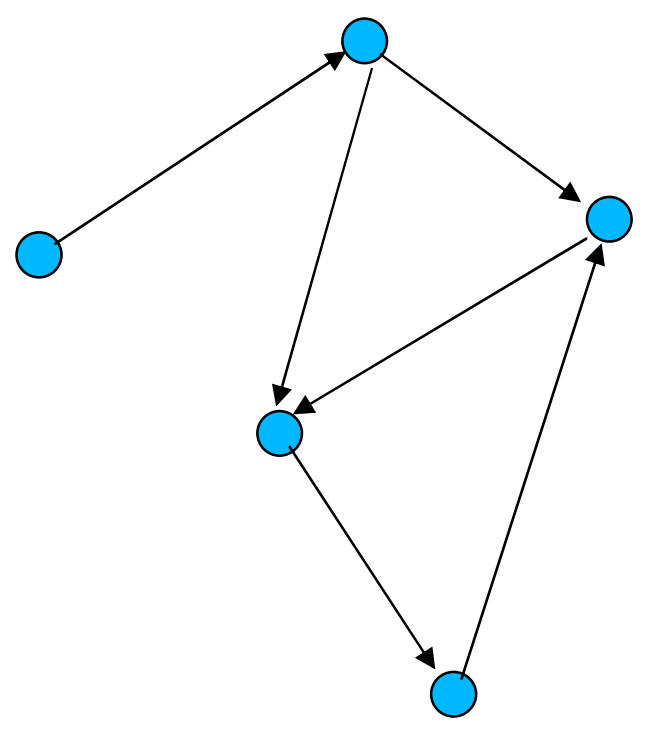

template <typename Graph >

struct boost::graph_traits \{

typedef ... vertex_descriptor;

typedef ... edge_descriptor;

typedef ... vertex_iterator;

typedef ... out_edge_iterator;

\} ; 


\section{BGL Glue Layer: Free Functions}

vertex_descriptor $\mathbf{v}$, w;

edge_descriptor e;

$v=\operatorname{source}(e, G) ;$

$\mathbf{w}=\operatorname{target}(\mathbf{e}, \mathbf{G})$;

std: :pair<out_edge_iterator, out_edge_iterator> ipair;

ipair = out_edges $(v, G)$;

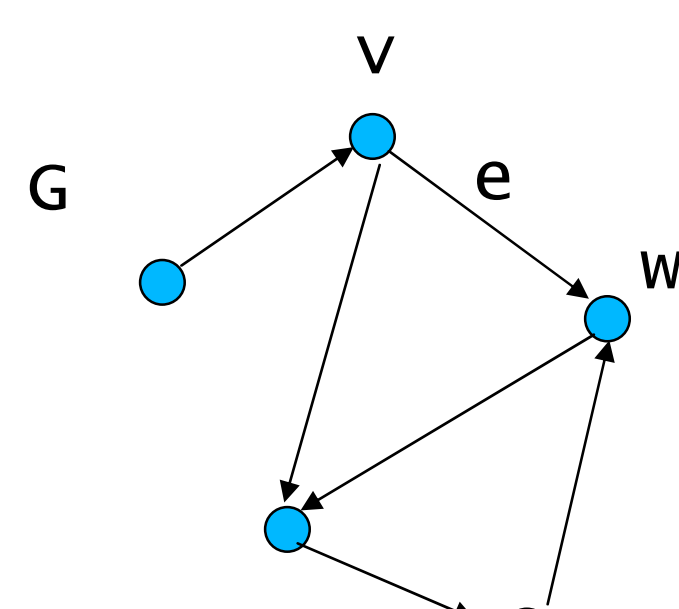




\section{BGL Glue Layer for CGAL}

CGAL provides partial specializations:

template <typename $T>$ graph_traits<Polyhedron $<T>>$;

template <typename $T>$ Polyhedron $\langle\mathrm{T}\rangle$ : : Vertex source (Polyhedron<T>: : Edge);

Users can run:

boost: : kruskal_mst (P) ;

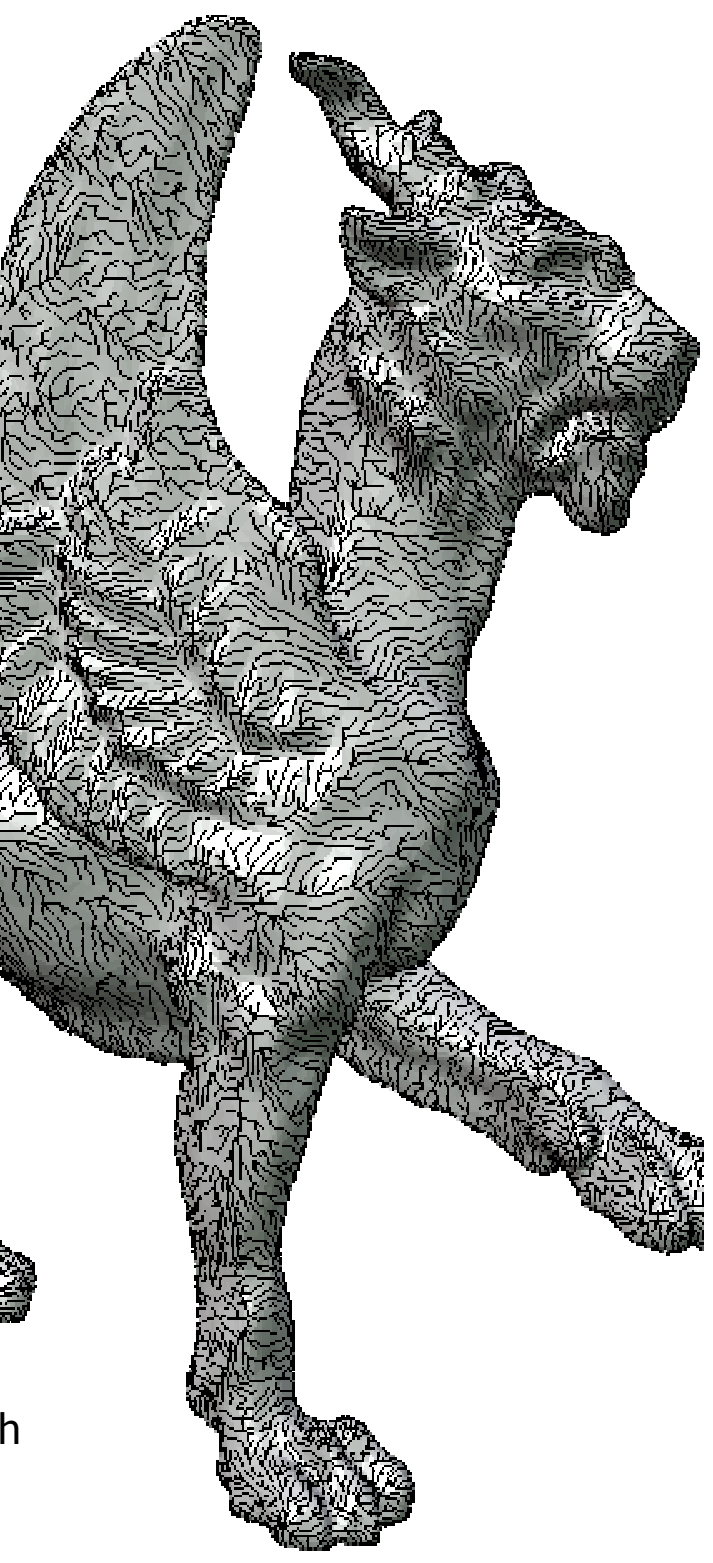




\section{From A BGL Glue Layer for CGAL}

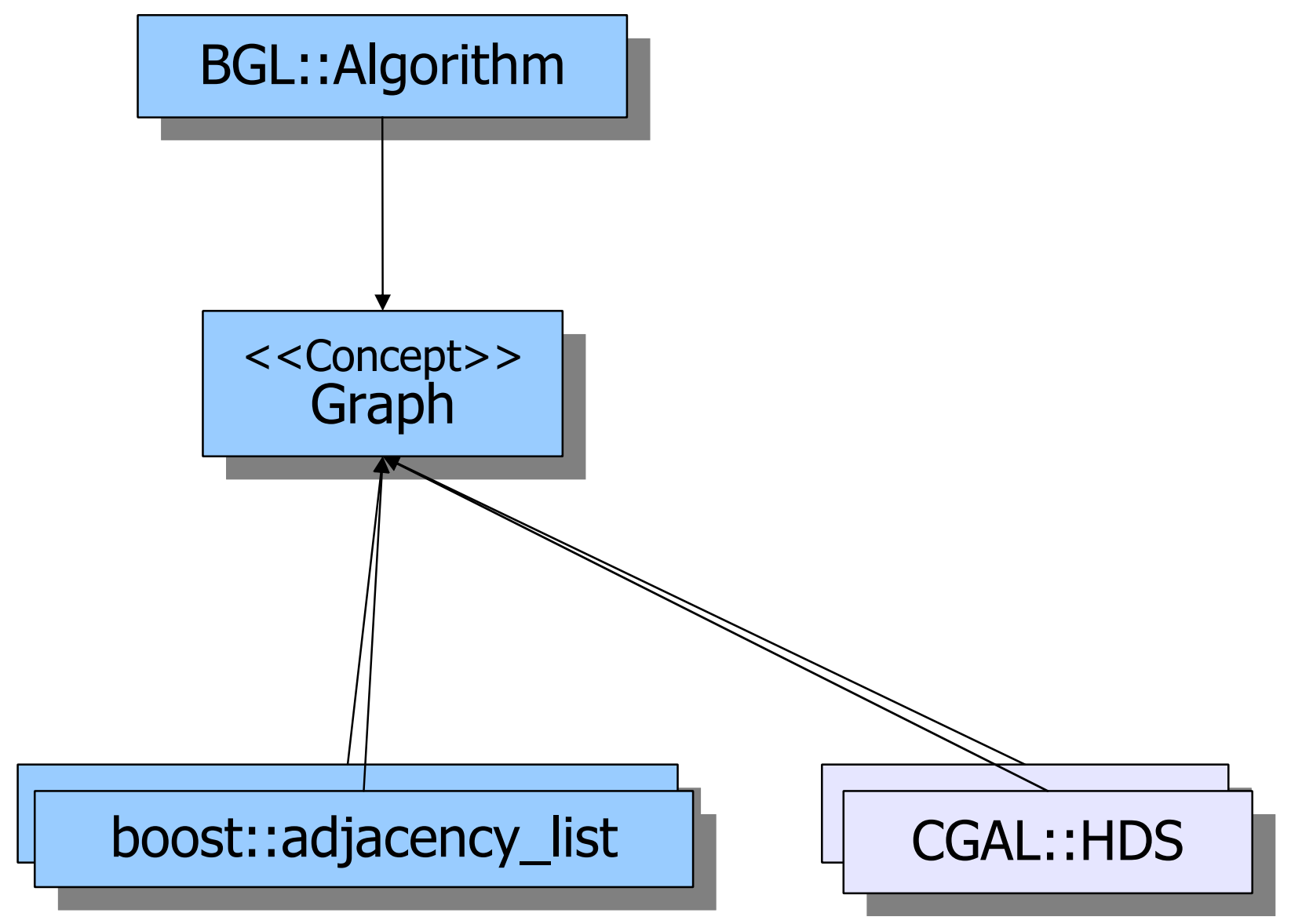




\section{To BGL Style CGAL Algorithms}

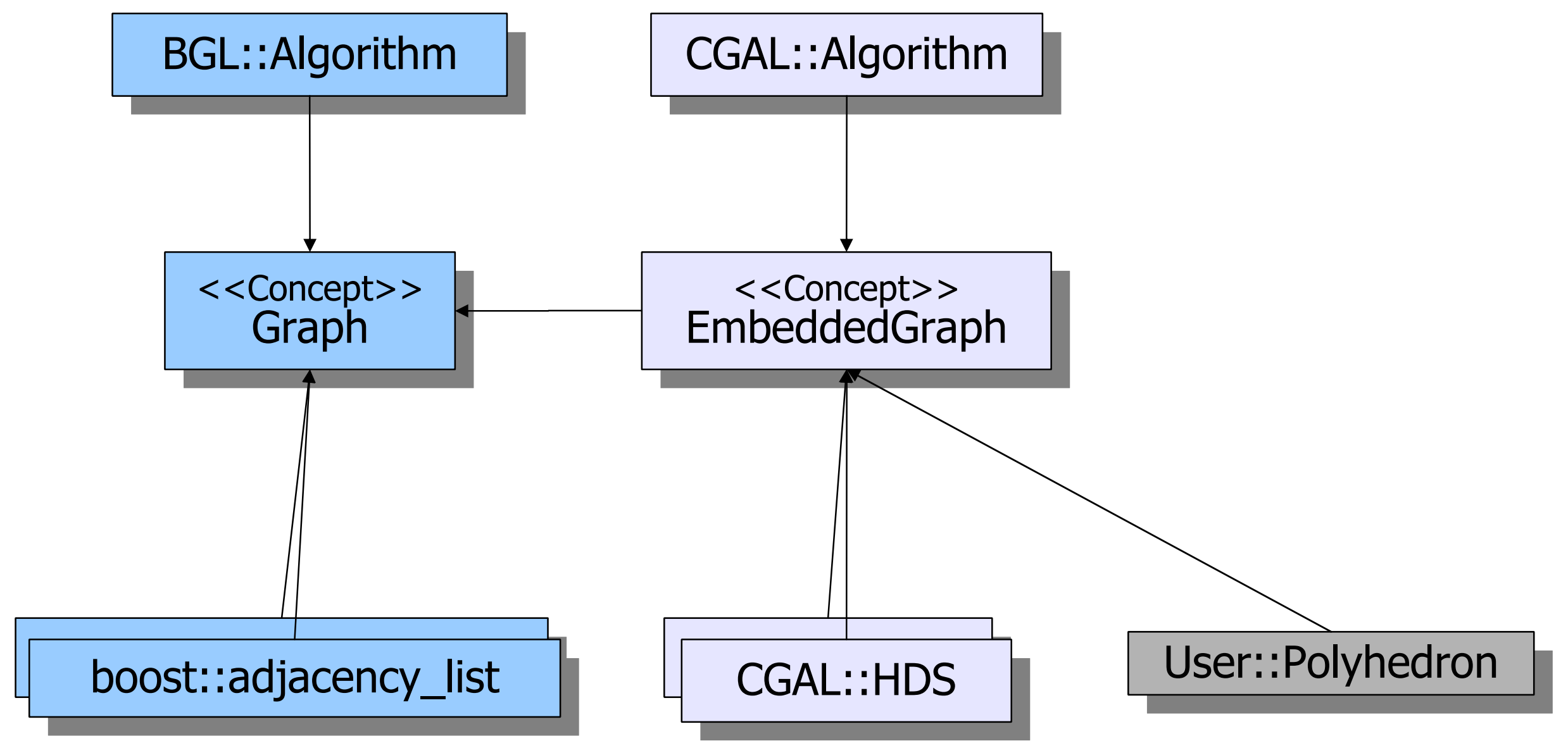




\section{Summary: Overview}

- Huge collection with uniform APIs

- Modular and not monolithic

- Open Source and commercial licenses

- Clear focus on geometry

- Interfaces with de facto standards/leaders: STL, Boost, GMP, Qt, blas

- Robust and fast through exact geometric computing

- Easy to integrate through generic programming 


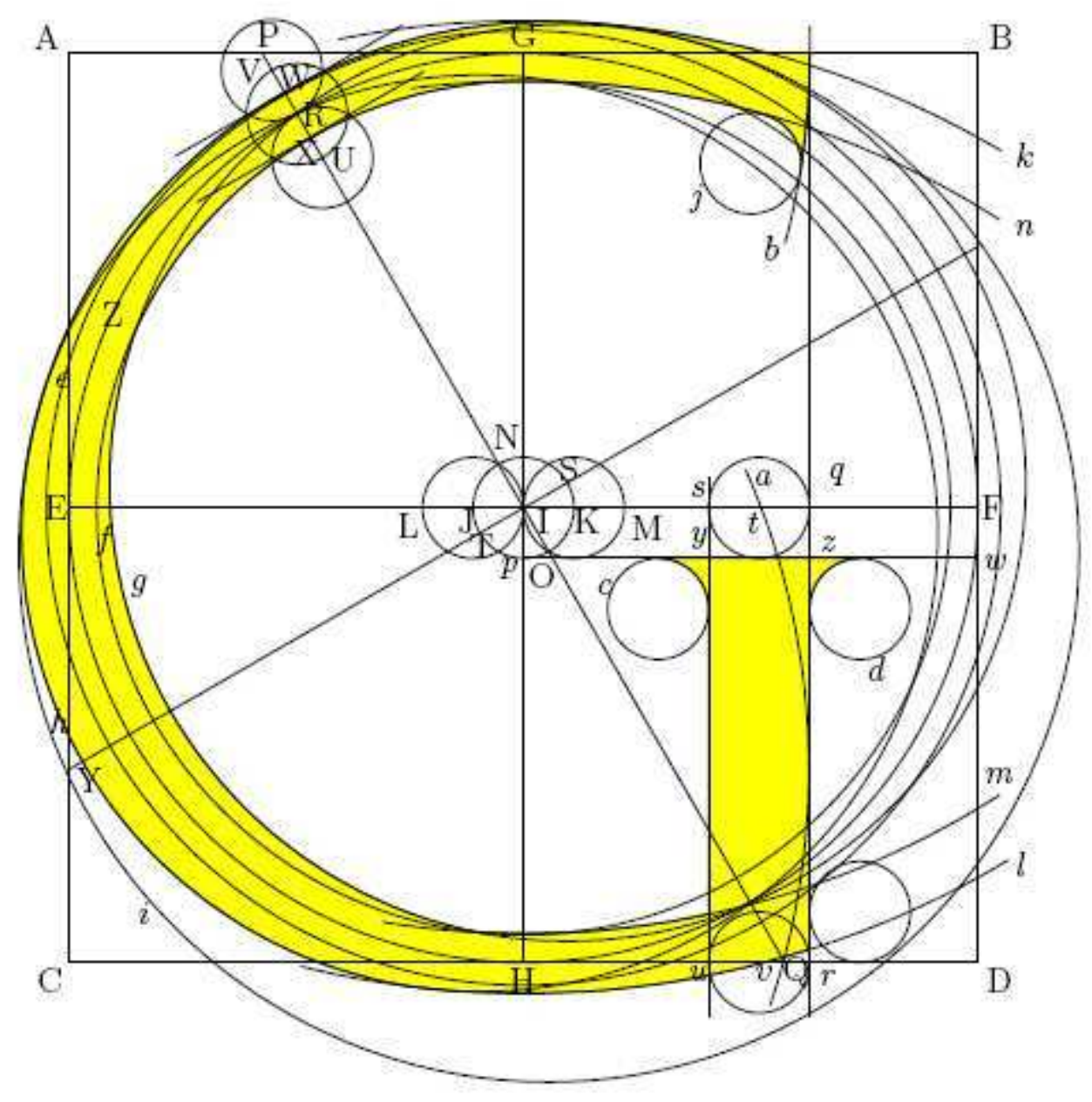

\title{
Polyhedral Surfaces
}

\author{
Pierre Alliez
}

INRIA 


\section{Outline}

- Halfedge Data Structure and Polyhedron

- Euler Operators

- Customization

- Algorithms for Geometric Modelling and Geometry Processing 


\section{Halfedge Data Structure}

Represented by vertices, edges, facets and an incidence relation on them, restricted to orientable 2-manifolds with boundary.
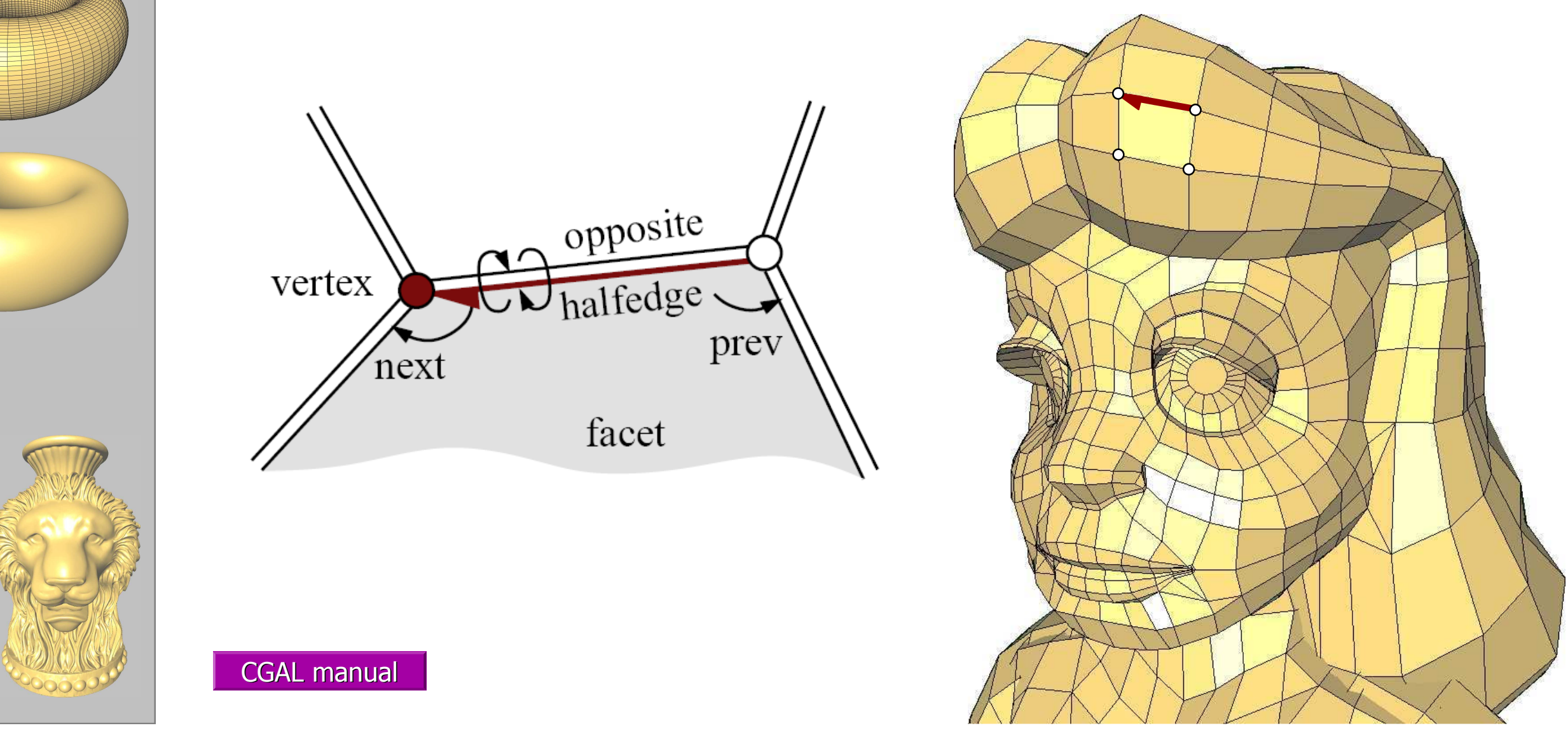


\section{Polyhedron}

Building blocks assembled with $\mathrm{C}++$ templates

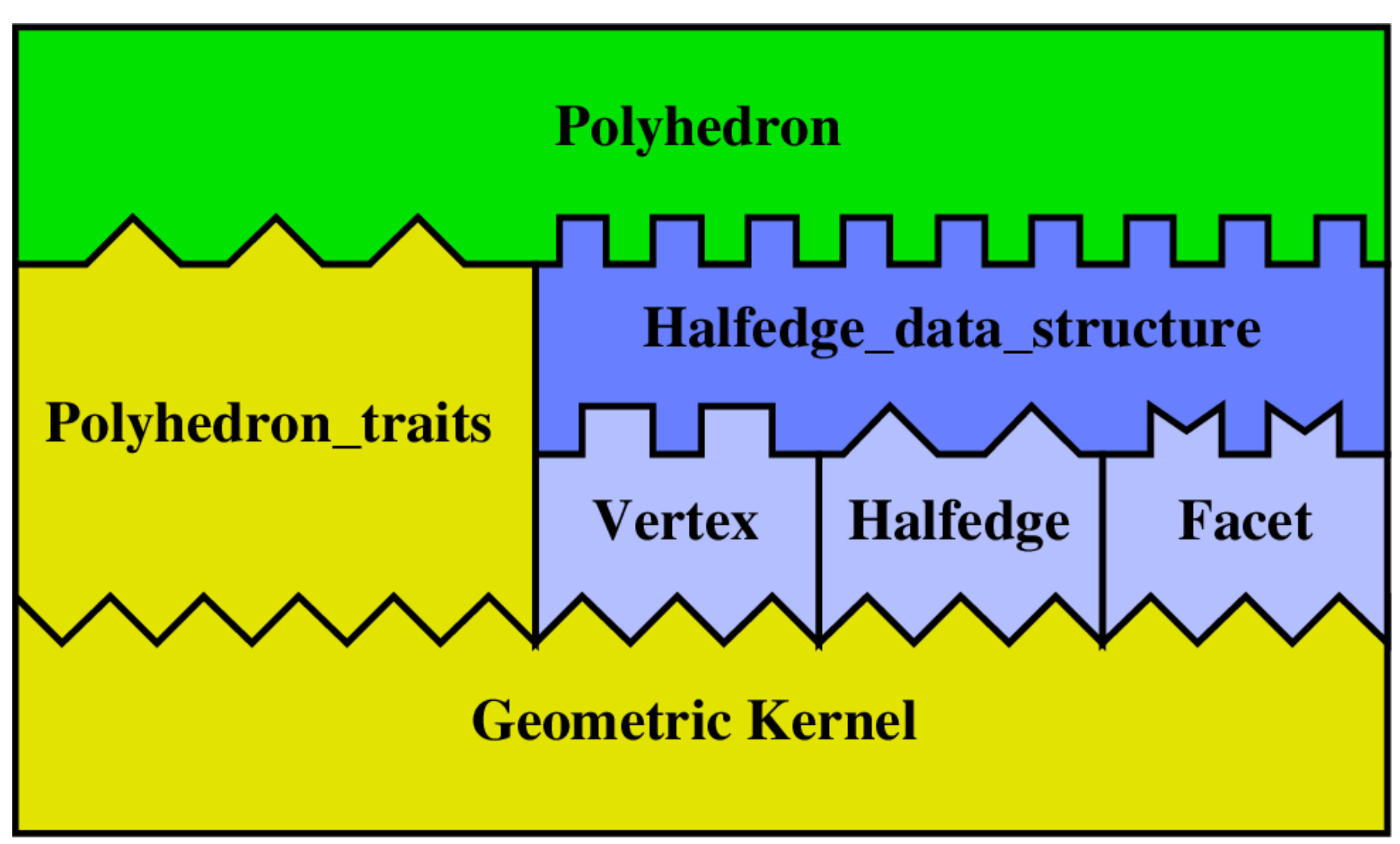




\section{Default Polyhedron}

\begin{tabular}{|ll|}
\hline Vertex & \\
\hline $\begin{array}{l}\text { Halfedge_handle } \\
\text { Point\& }\end{array}$ & $\begin{array}{l}\text { halfedge() } \\
\text { point }()\end{array}$ \\
\hline$\ldots \ldots$ & $\ldots$ \\
\hline
\end{tabular}

\begin{tabular}{|ll|}
\hline Halfedge \\
\hline Halfedge_handle & opposite() \\
Halfedge_handle & next() \\
Halfedge_handle & $\operatorname{prev}()$ \\
Vertex_handle & vertex() \\
Facet_handle & facet() \\
\hline$\ldots \ldots .$. & $\ldots$ \\
\hline
\end{tabular}

\begin{tabular}{|ll|}
\hline Facet & \\
\hline Halfedge_handle & halfedge() \\
Plane\& & plane() \\
\hline Normal\& & normal() \\
Color\& & color() \\
$\ldots . .$. & $\ldots$ \\
\hline
\end{tabular}

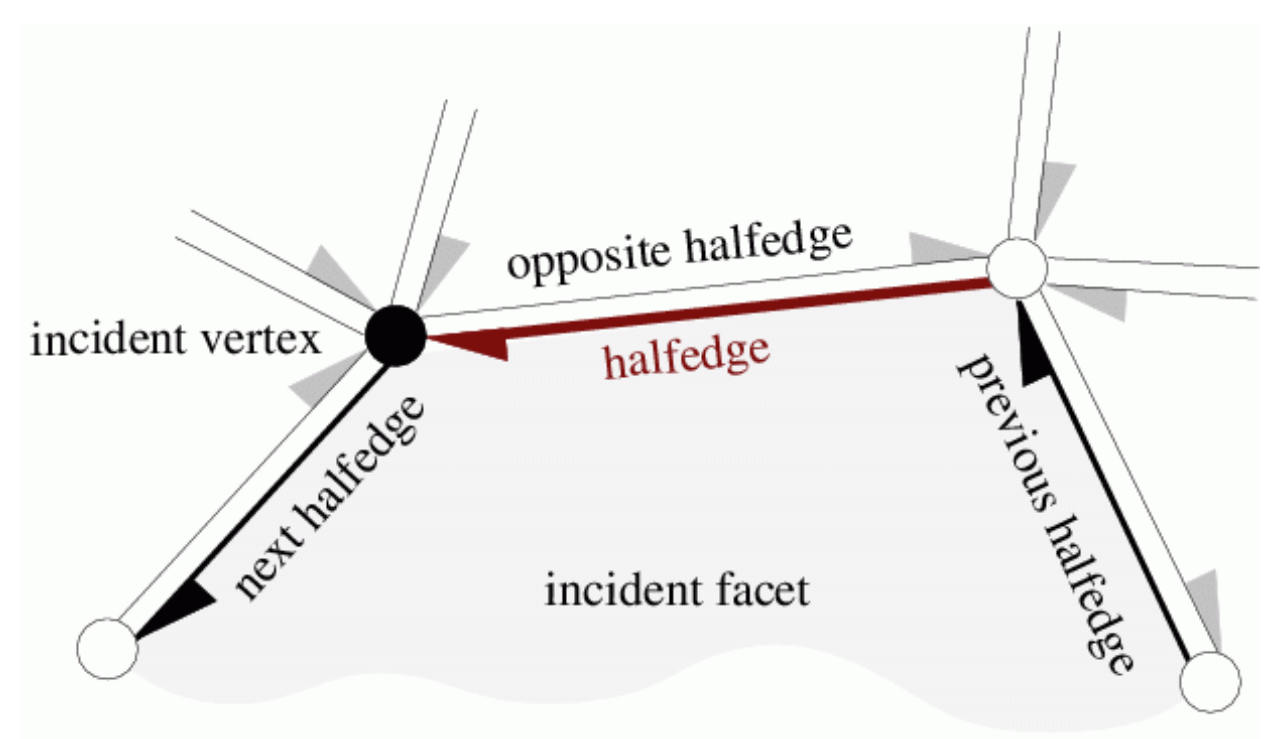

CGAL manual 


\section{Example}

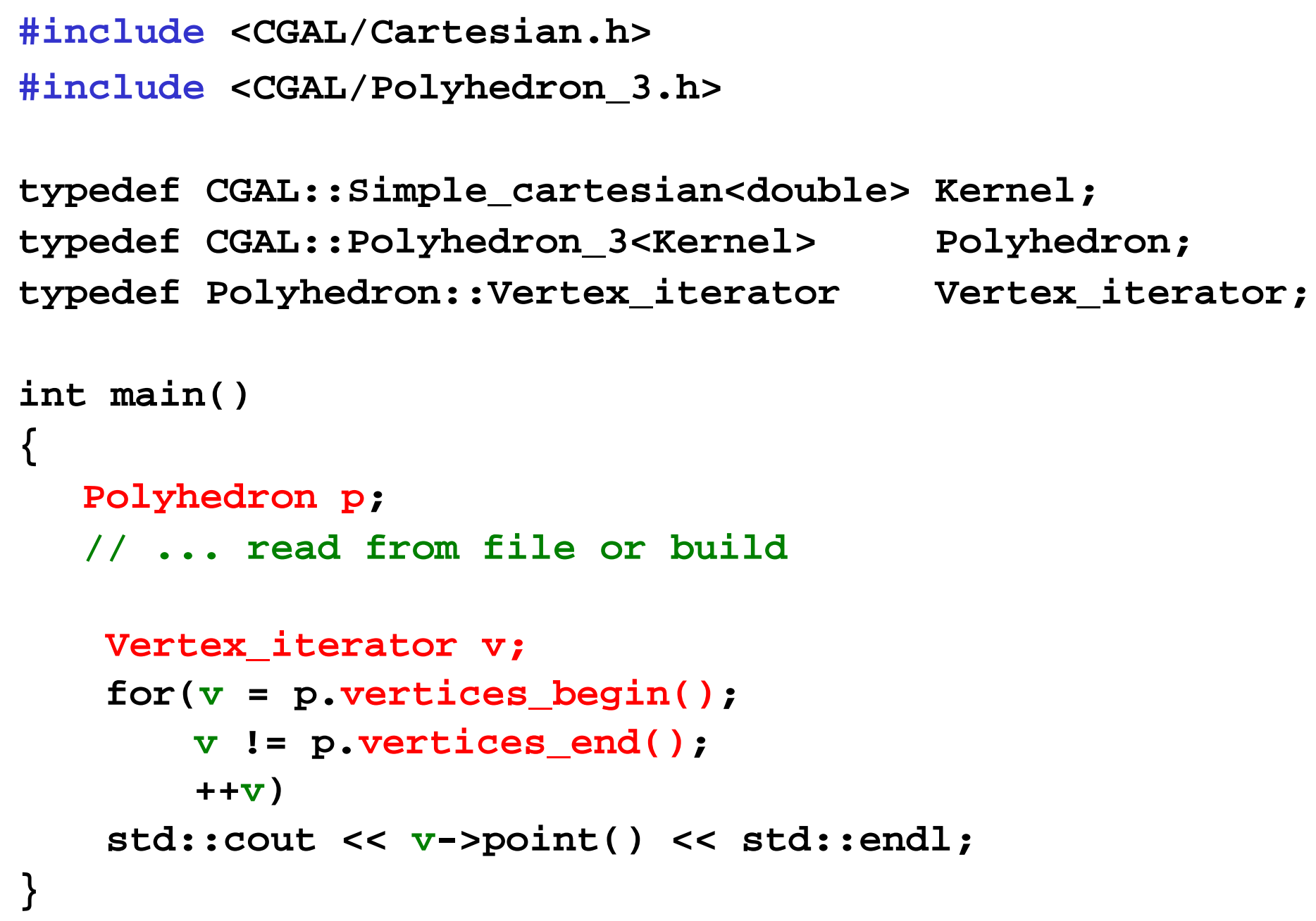




\section{Flexible Data Structure}

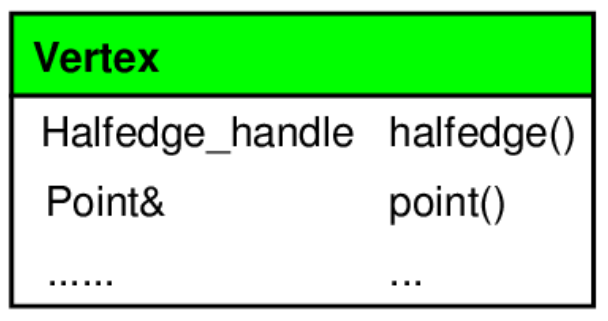

\begin{tabular}{|ll|}
\hline Halfedge & \\
\hline Halfedge_handle & opposite () \\
Halfedge_handle & next() \\
\hline Halfedge_handle & $\operatorname{prev}()$ \\
Vertex_handle & vertex() \\
Facet_handle & facet() \\
$\ldots . .$. & $\ldots$ \\
\hline
\end{tabular}

\begin{tabular}{|ll|}
\hline Facet & \\
\hline Halfedge_handle & halfedge() \\
Plane\& & plane() \\
Normal\& & normal() \\
Color\& & color() \\
$\ldots \ldots$ & $\ldots$ \\
\hline
\end{tabular}

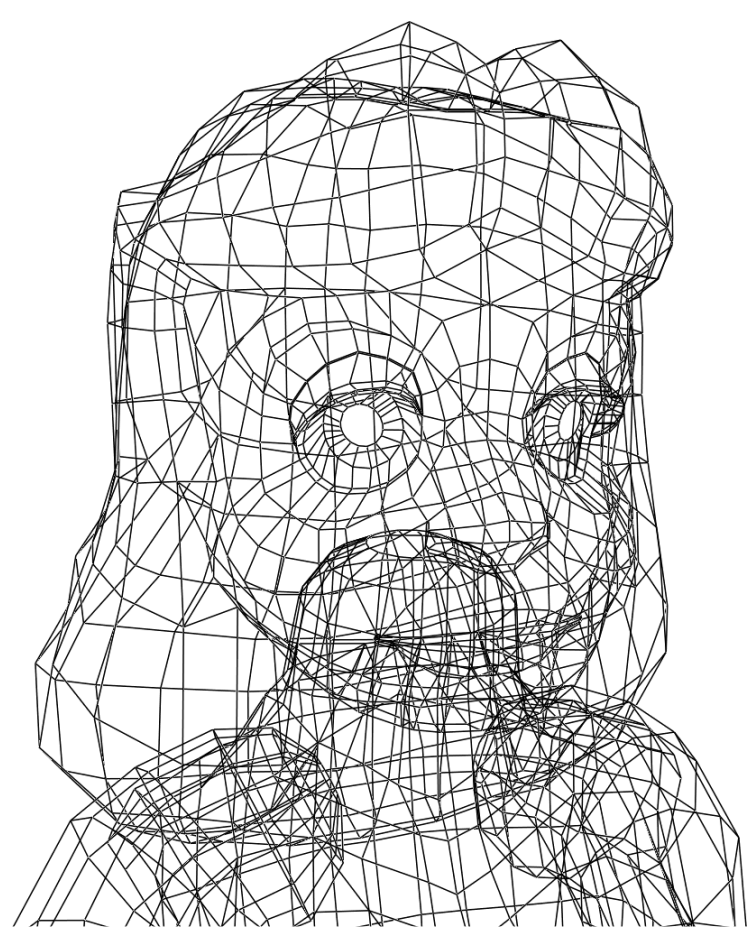




\section{Iterate over all Vertices}

Vertex_iterator $\mathbf{v}$;

for ( $\mathbf{v}=$ polyhedron.vertices_begin();

v ! = polyhedron.vertices_end ();

$++v$ )

\{

// do something with $v$ \}

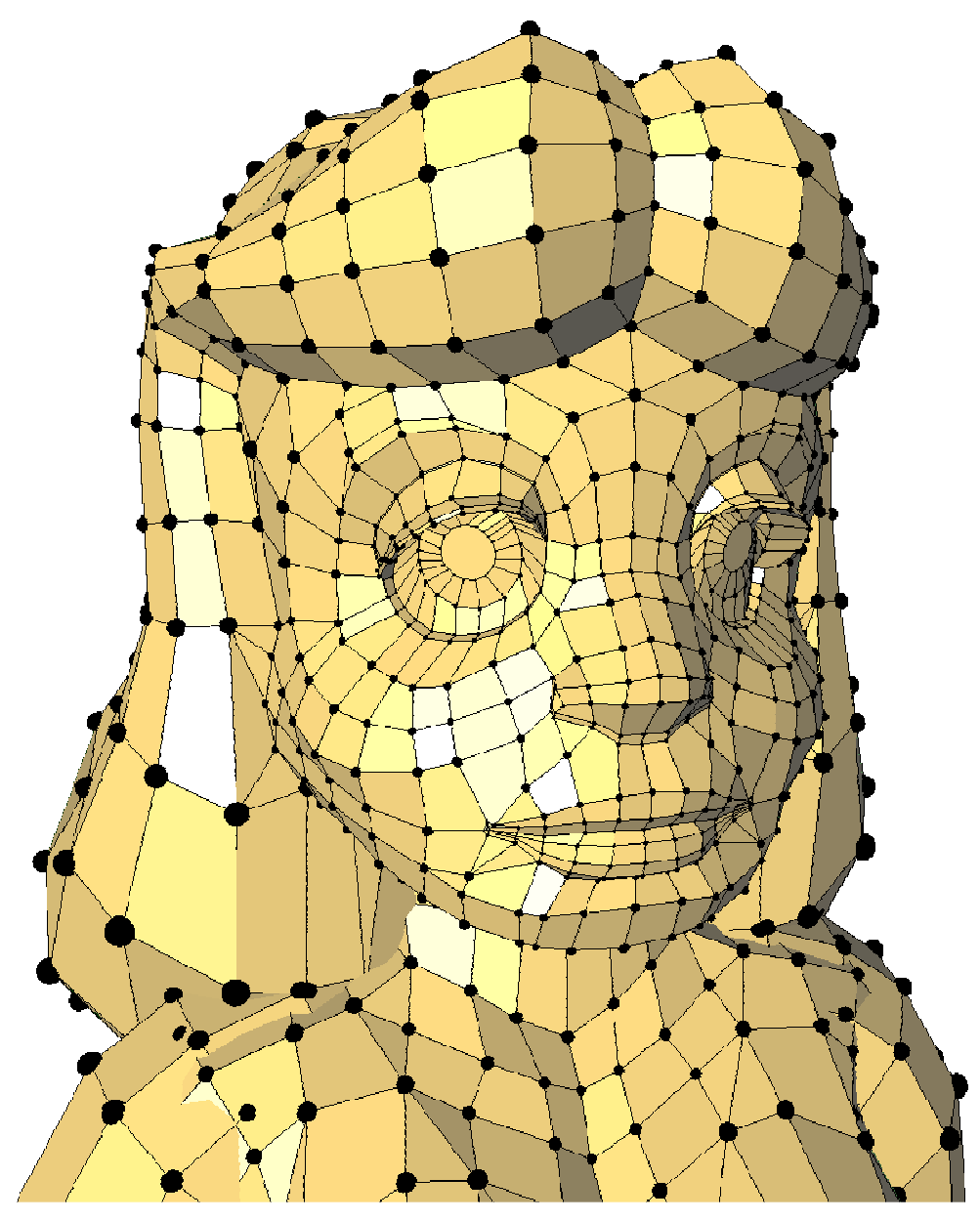




\section{Circulate around Facet}

Halfedge_around_facet_circulator he, end; he $=$ end $=$ f->facet_begin (); CGAL_For_all (he, end)

f

// do something with he \}

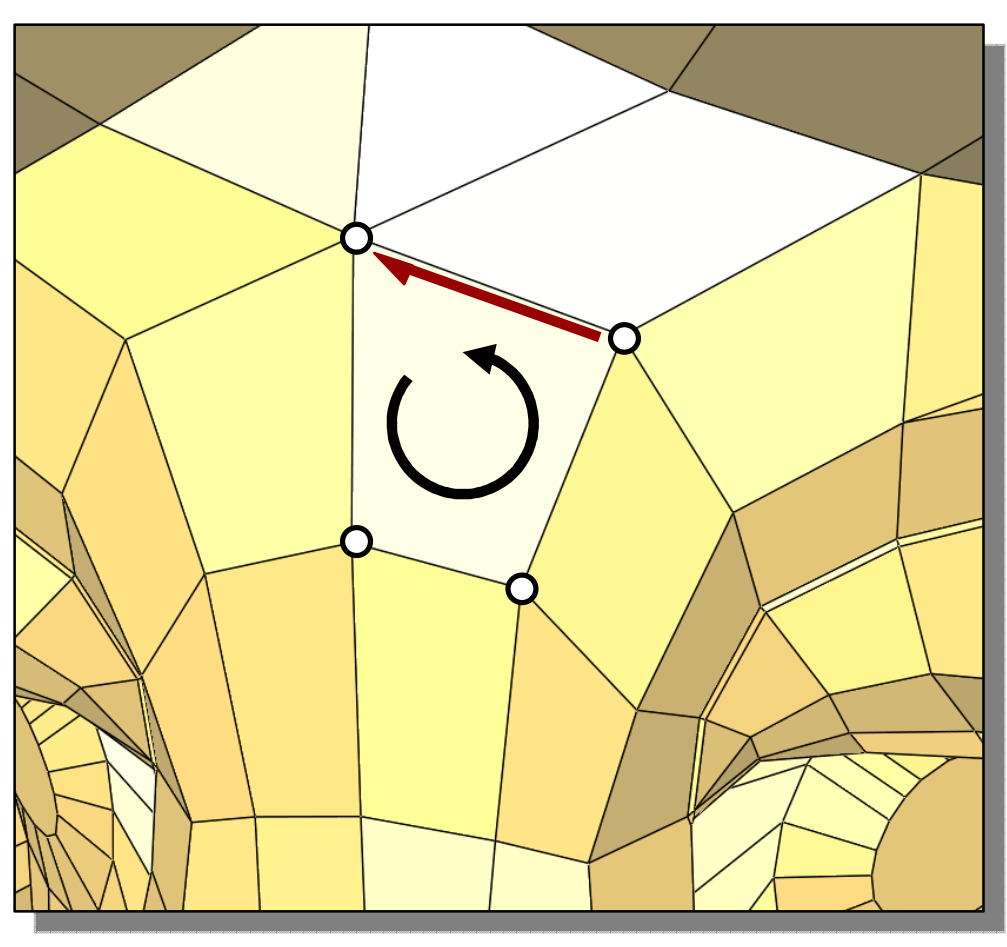




\section{Circulate around Vertex}

Halfedge_around_vertex_circulator he, end; he $=$ end $=$ v->vertex_begin () ; CGAL_For_all (he, end) \{

// do something with he \}

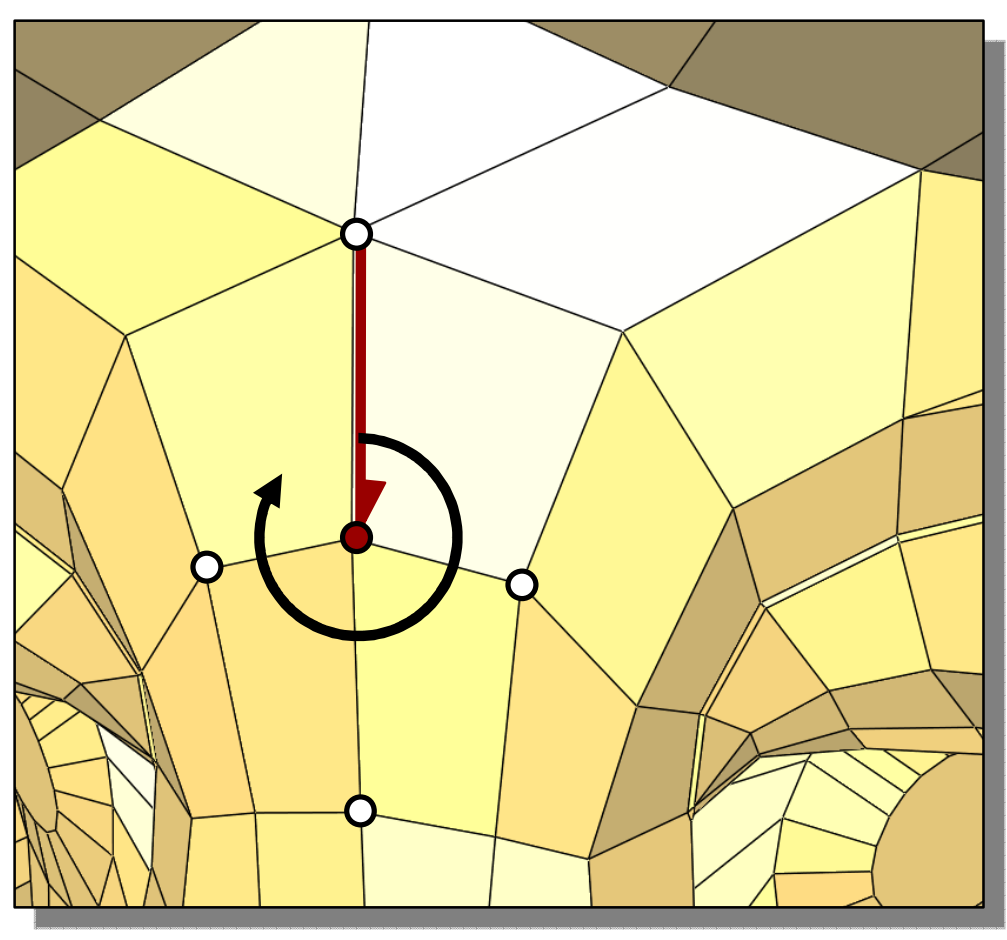




\section{Euler Operators}

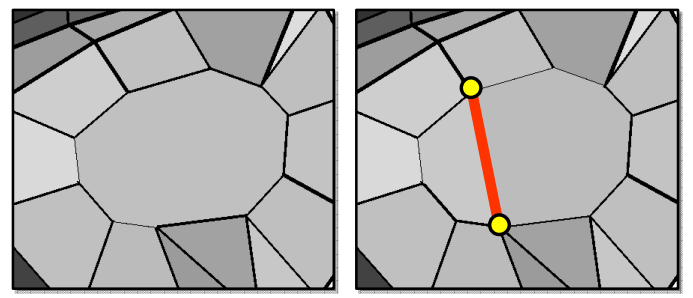

split_facet join_facet

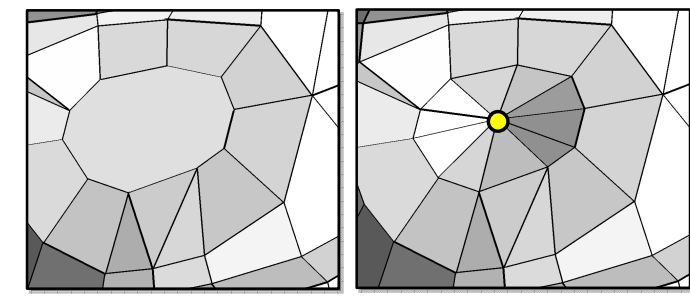

create_center_vertex erase_center_vertex

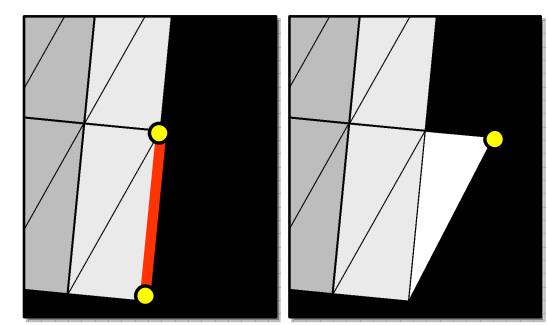

add_vertex_and_facet to_border erase_facet

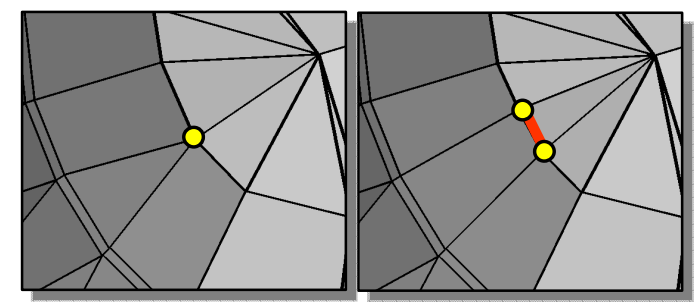

split_vertex join_vertex (aka edge collapse)

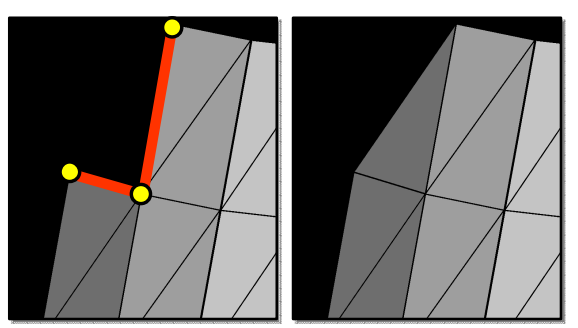

add_facet_to_border erase_facet 


\section{Customization}
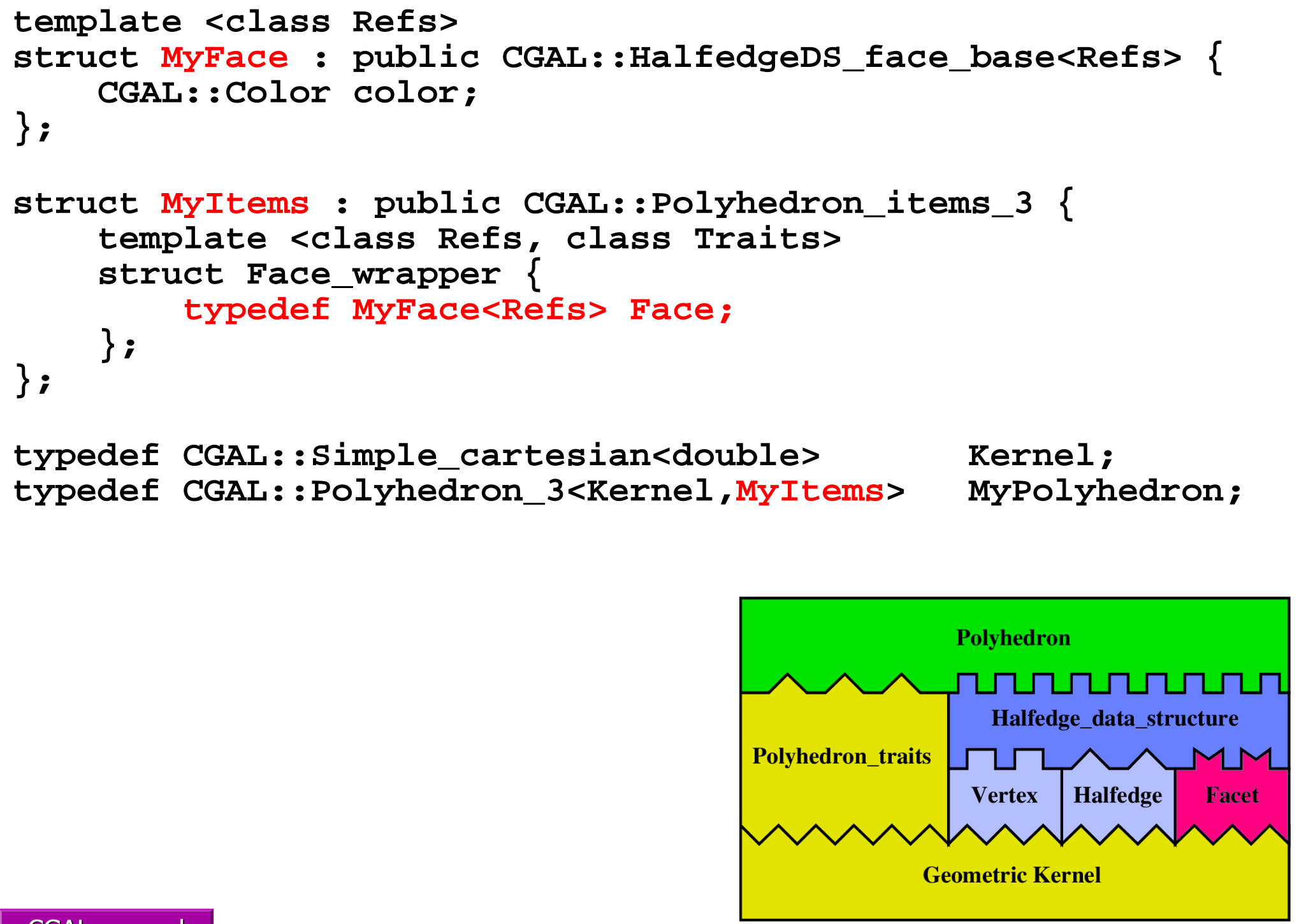


\section{Algorithms}

- Intersection detection

- Convex hull

- Boolean operations

- Kernel

- Parameterization

- Subdivision

- Principal component analysis

- Estimation of curvatures

- Extraction of ridges

- Simplification 


\section{Intersection Detection}

Efficient algorithm for finding all intersecting pairs for large numbers of axis-aligned bounding boxes.

Generic programming: Boxes can contain

R. objects of any type

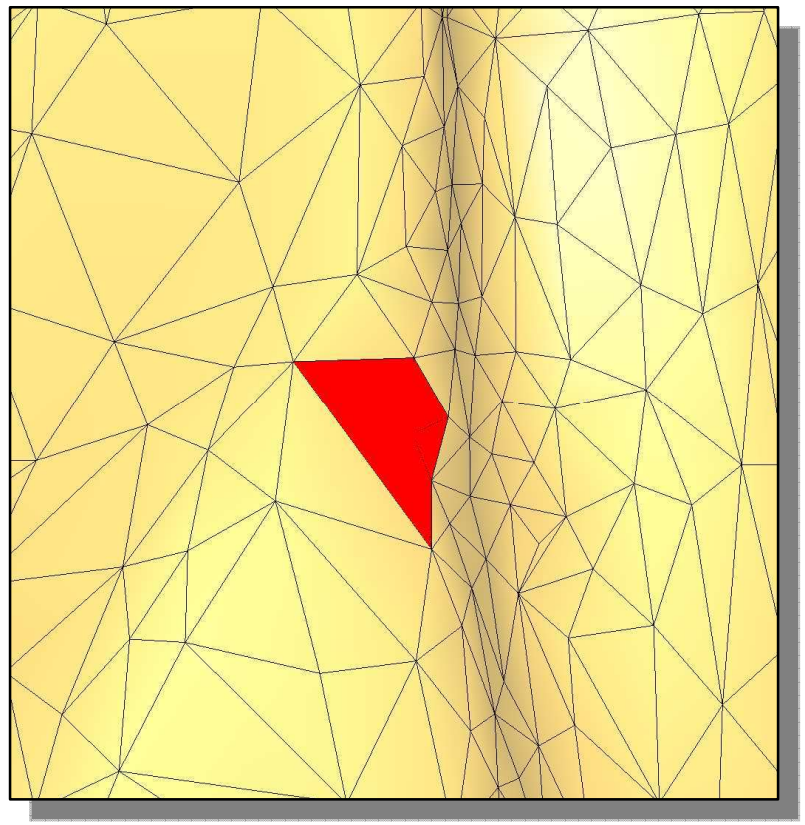




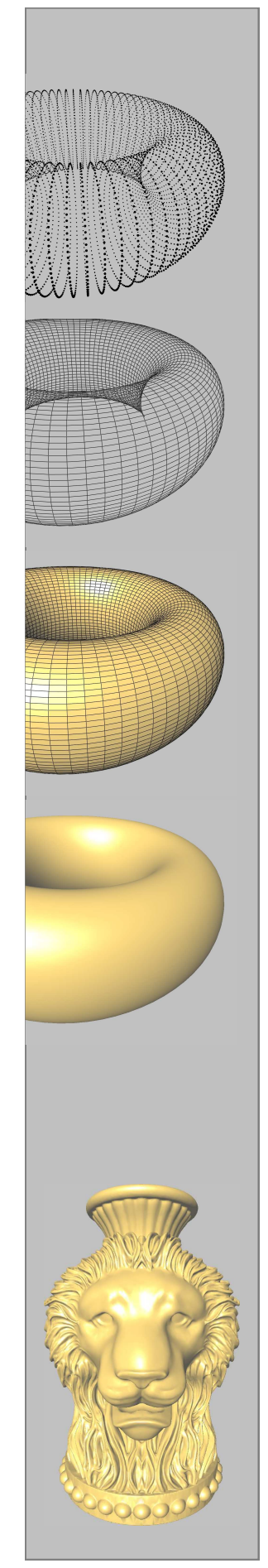

\section{Example: Intersecting 3D Triangles}

\#include <CGAL/Exact_predicates_inexact_constructions_kernel.h>

\#include <CGAL/intersections.h>

\#include <CGAL/box_intersection_d.h>

typedef CGAL: :Exact_predicates_inexact_constructions_kernel Kernel;

typedef Kernel::Triangle_3 Triängle_3;

typedef std: :list<Triangle_3>::iterator Iterator;

typedef CGAL::Box_intersection_d::Box_with_handle_d<double, 3 , Iterator $>$ Box;

void callback (const Box\& $a$, const Box\& b)

\{

Triangle_3 ta $=* a$.handle ();

Triangle_3 tb $=\star b$.handle ();

if (CGAL: : do_intersect $(t a, t b))$ \{

CGAL : :box_self_intersection_d(boxes.begin(), boxes.end(), callback); 


\section{Convex Hull}

- From point set

- Outputs a polyhedron

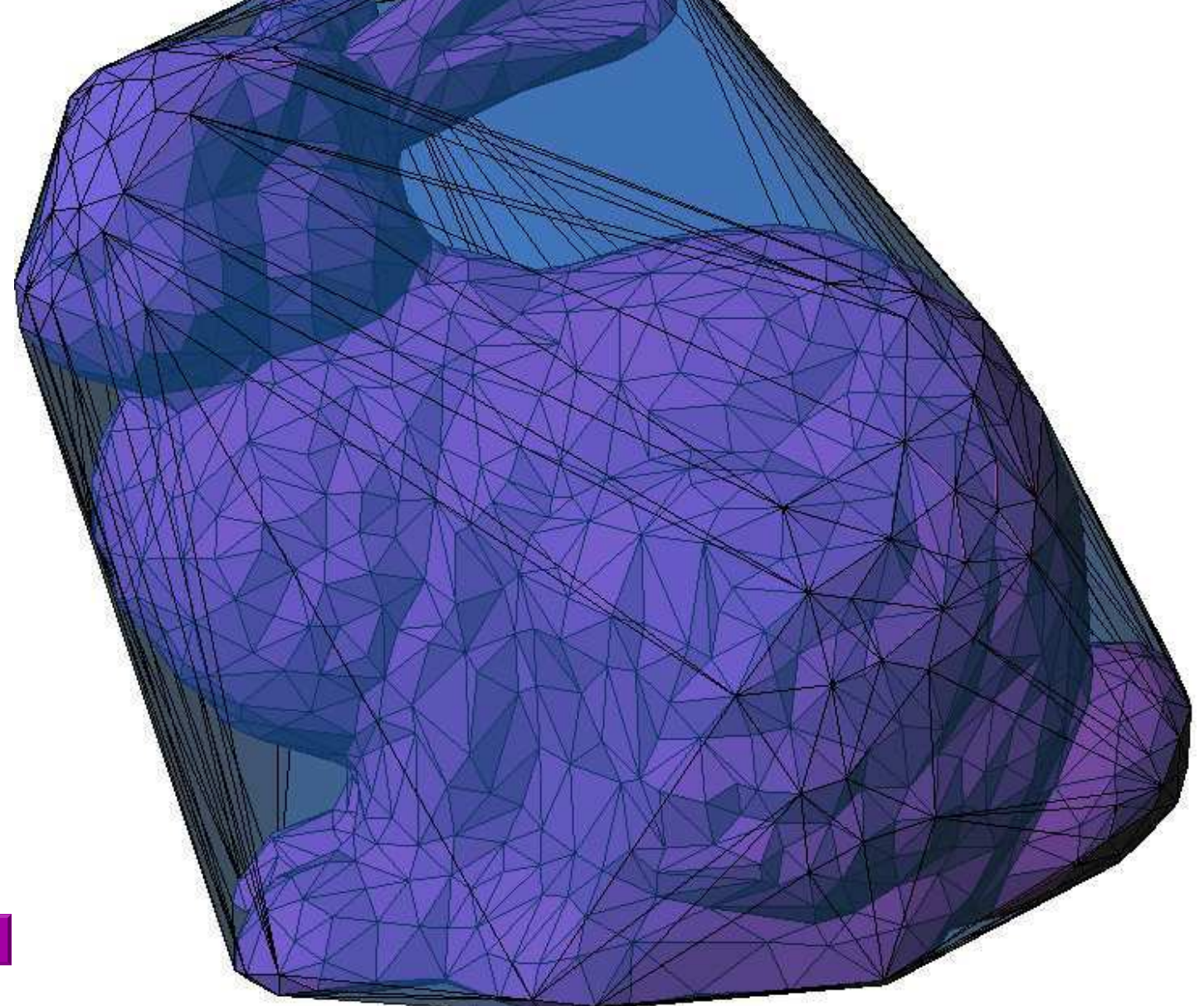




\section{Example}

\#include <CGAL/convex_hull_3.h>

\#include <CGAL/ Exact_predicates_inexact_constructions_kernel.h>

typedef CGAL: :Exact_predicates_inexact_constructions_kernel Kernel;

typedef CGAL: :Convex_hull_traits_3<Kernel> Traits;

typedef Traits::Polyhedron_3 Polyhedron;

typedef Kernel::Point_3 Point;

int main()

\{

std: :list<Point> points;

// fill container...

Polyhedron polyhedron;

CGAL : :convex_hull_3 (points.begin(), points.end(), polyhedron) ; return 0 ;

\} 


\section{Boolean Operations}

\section{Operations:}

- Union

- Difference

- Intersection

- Complement

Problem: not closed, i.e., result of a Boolean operation is not necessarily a Polyhedron
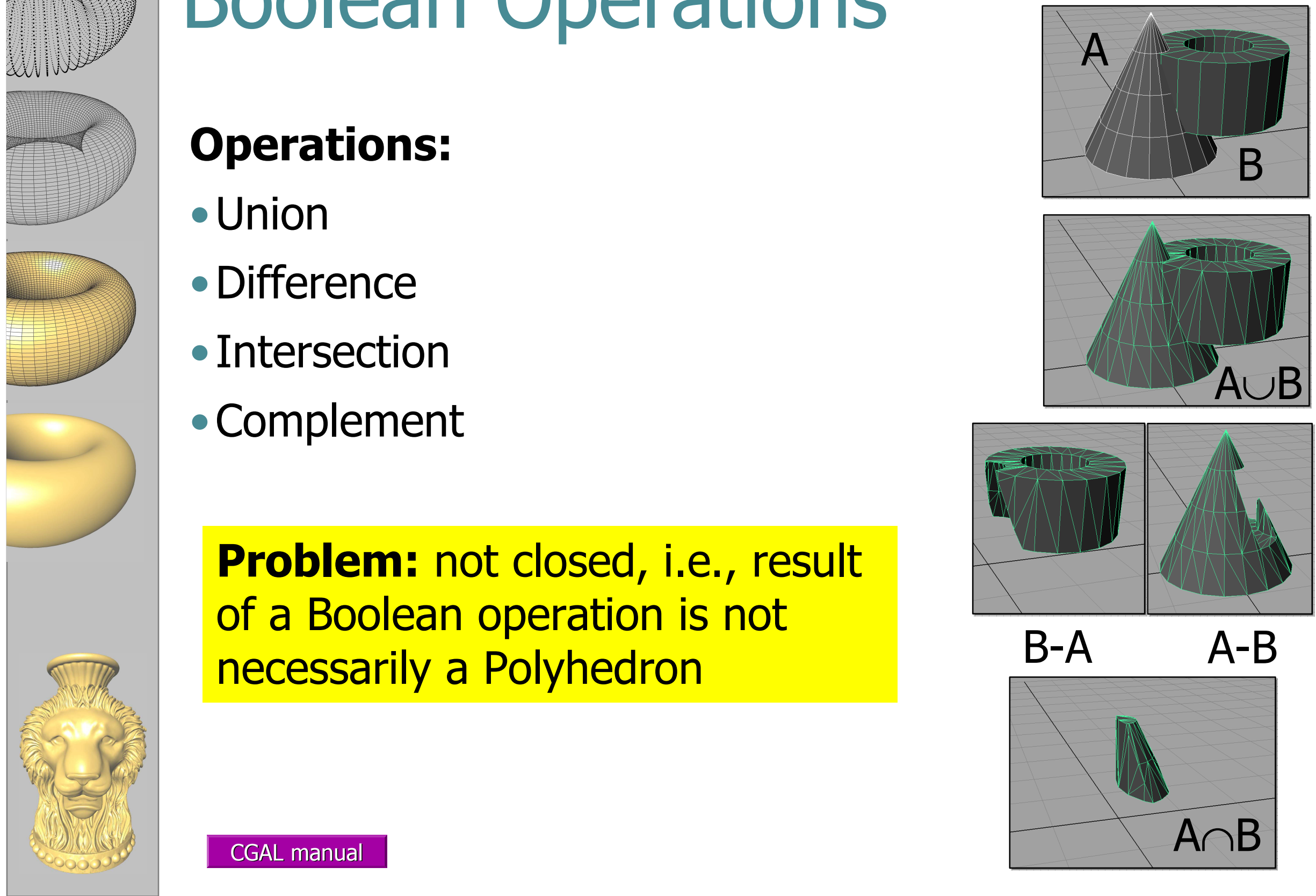


\section{Nef Polyhedron}

The 3D Nef polyhedron is a B-rep data structure which is

- closed under Boolean operations

- without enforcing regularization

Operations:

- Union

- Intersection

- Difference

- Complement

- Interior

- Exterior

- Boundary

- Closure

- Regularization

can evaluate a CSGtree with halfspaces as primitives and convert it to B-rep 


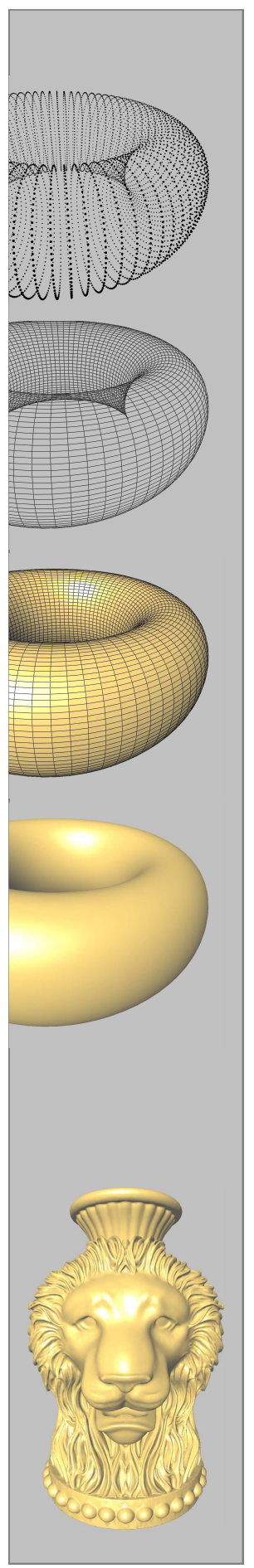

\section{Example}

\#include <CGAL/Exact_predicates_exact_constructions_kernel.h> \#include <CGAL/Polyhedron_3.h>

\#include <CGAL/Nef_polyhedron_3.h>

typedef CGAL::Exact_predicates_exact_constructions_kernel Kernel; typedef CGAL: :Polyhedron_3<Kernel> Polyhedron;

typedef CGAL: :Nef_polyhedron_3<Kernel> Nef_polyhedron;

Polyhedron p1;

Polyhedron p2;

Nef_polyhedron n1 (p1); Nef_polyhedron n2 (p2);

$\mathrm{n} 1-=\mathrm{n} 2 ; / /$ difference

Polyhedron p3;

if (n1.is_simple ())
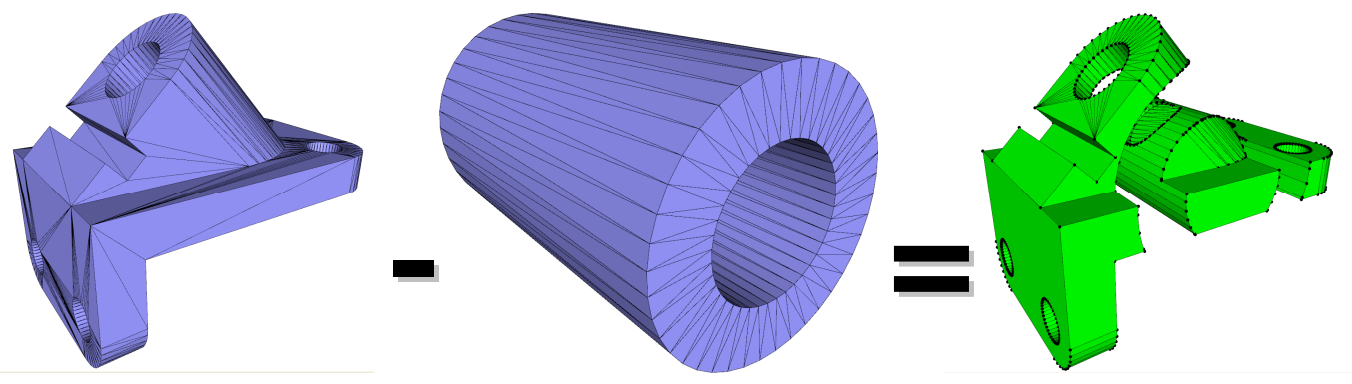
else

n1.convert_to_Polyhedron (p3);

// analyze/process $\mathrm{n} 1$ and do something... 


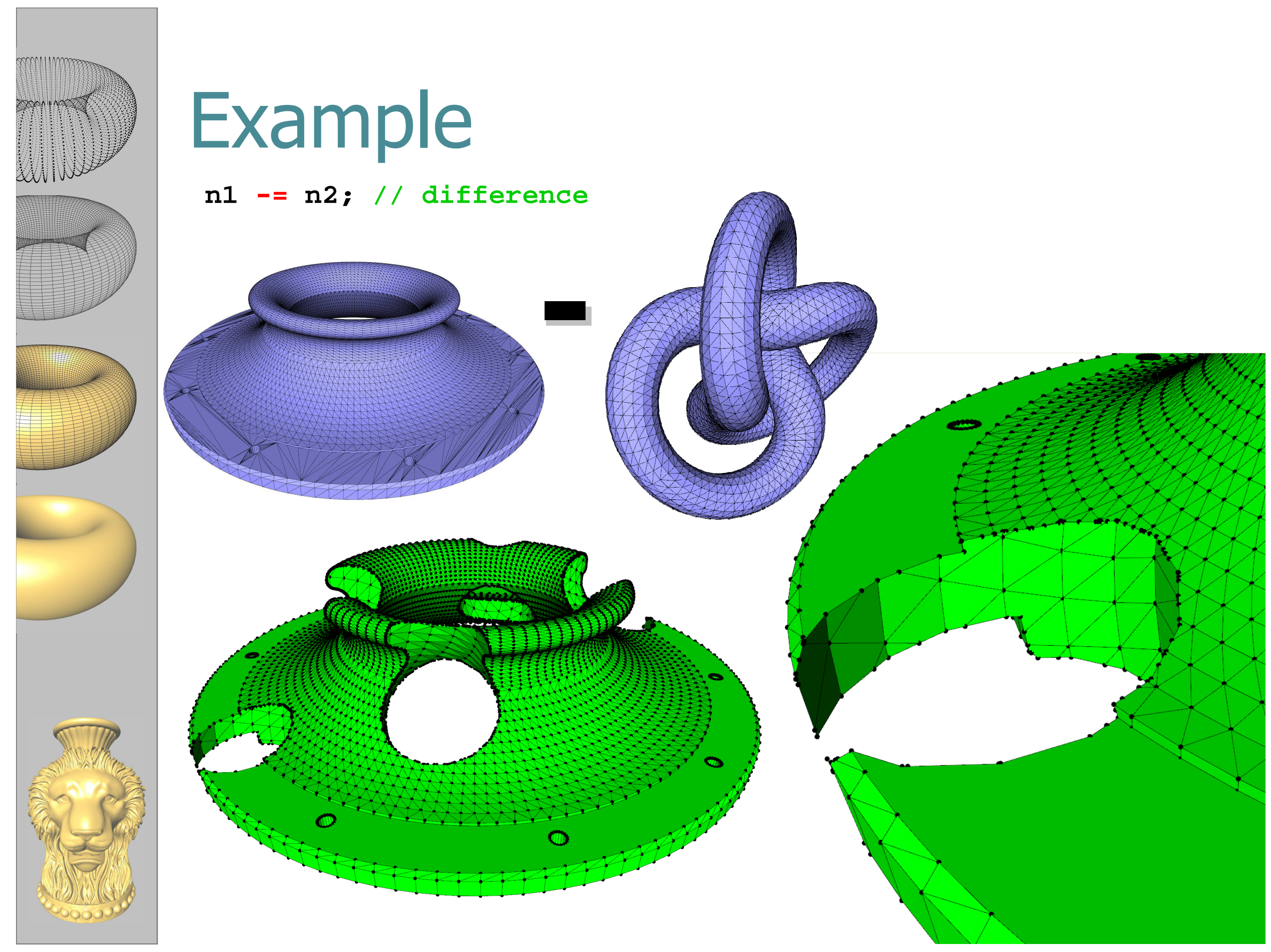




\section{Kernel of a Polyhedron}

- Intersection of all its interior half-spaces.

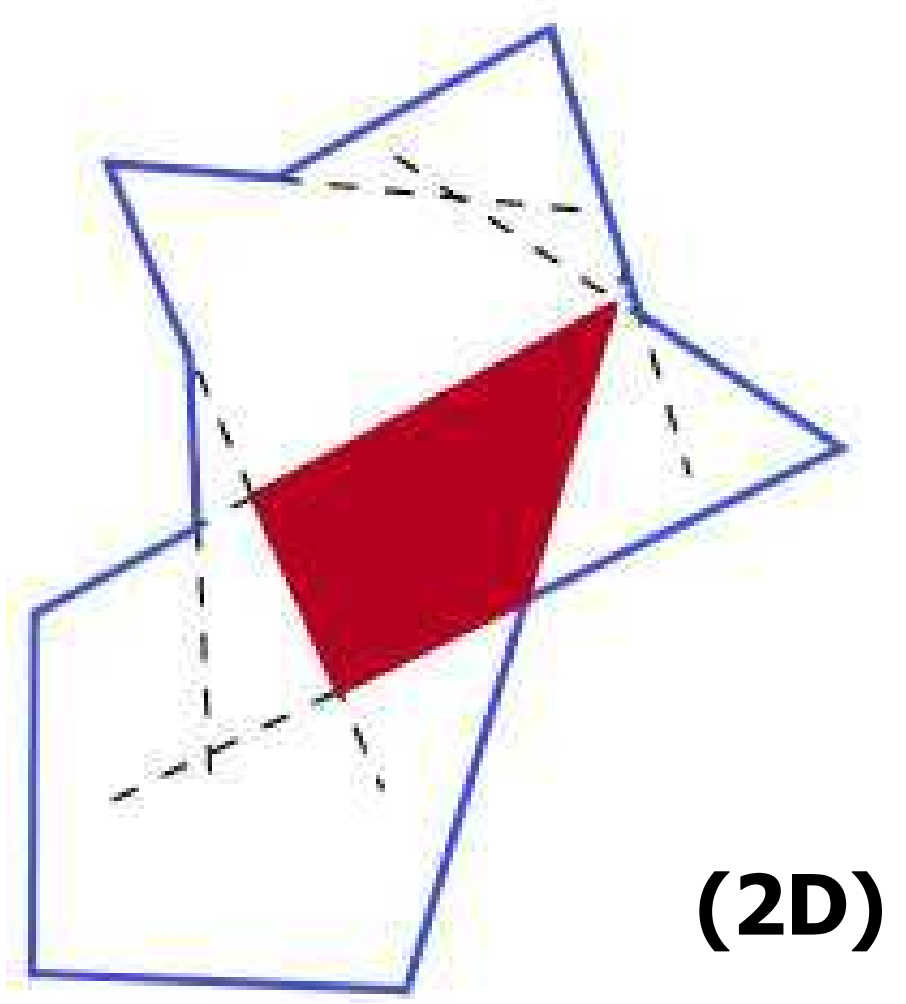




\section{Kernel of a Polyhedron}

- Intersection of all its interior half-spaces

- Uses linear programming (CGAL::QP_solver)

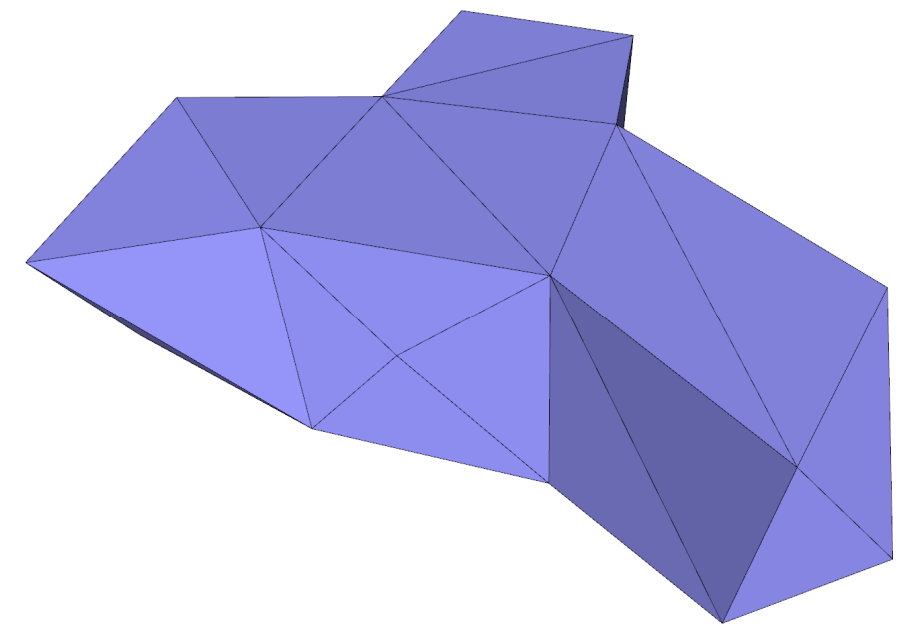

input polyhedron

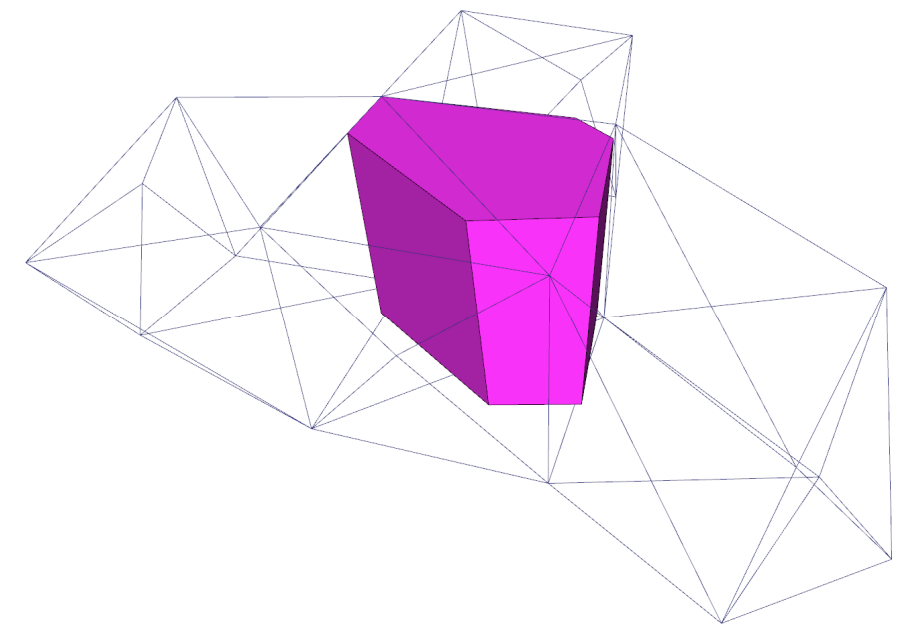

kernel 


\section{Kernel w/ Linear Programming}

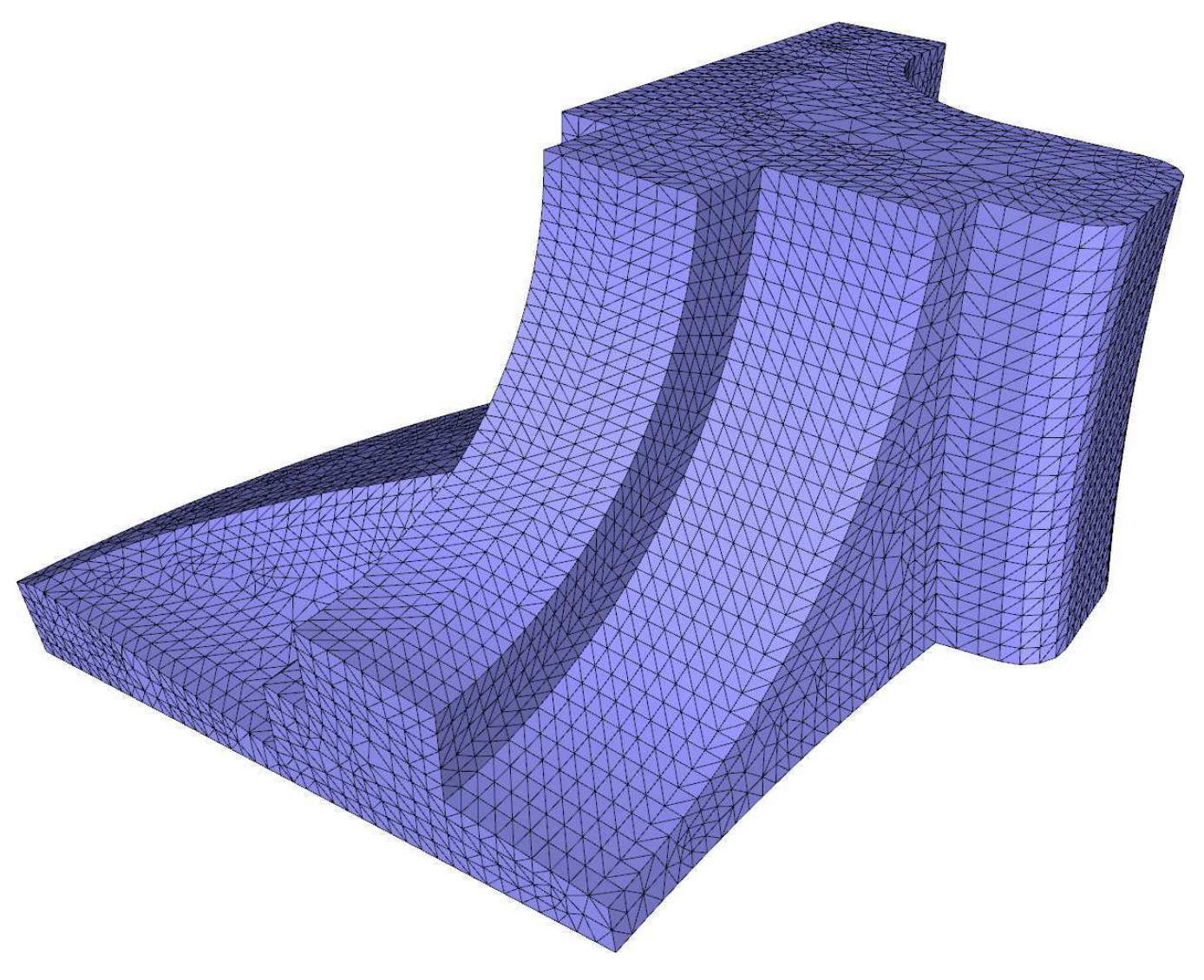

Does it have a kernel? 


\section{Kernel w/ Linear Programming}

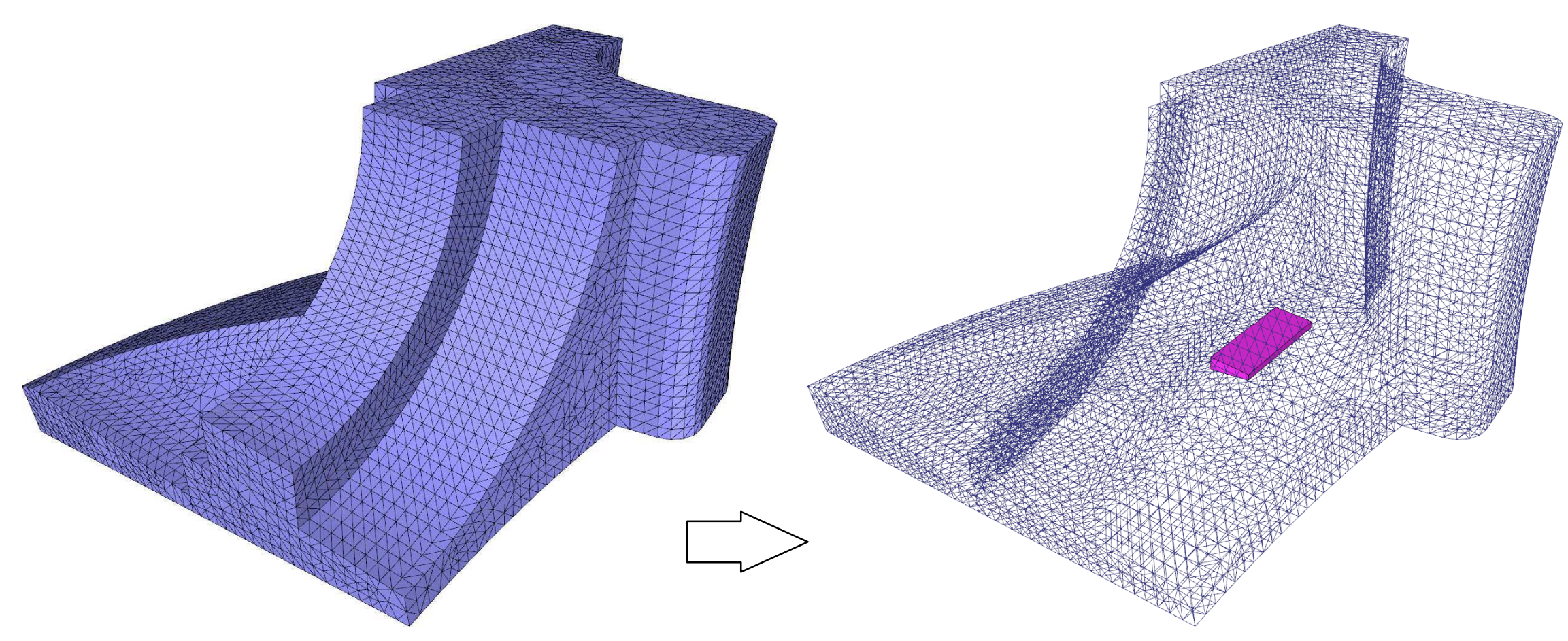




\section{Parameterization}

\section{Planar}

- Conformal [Eck et al., Levy et al., Desbrun et al.]

- Mean value coordinates [Floater]

- ...
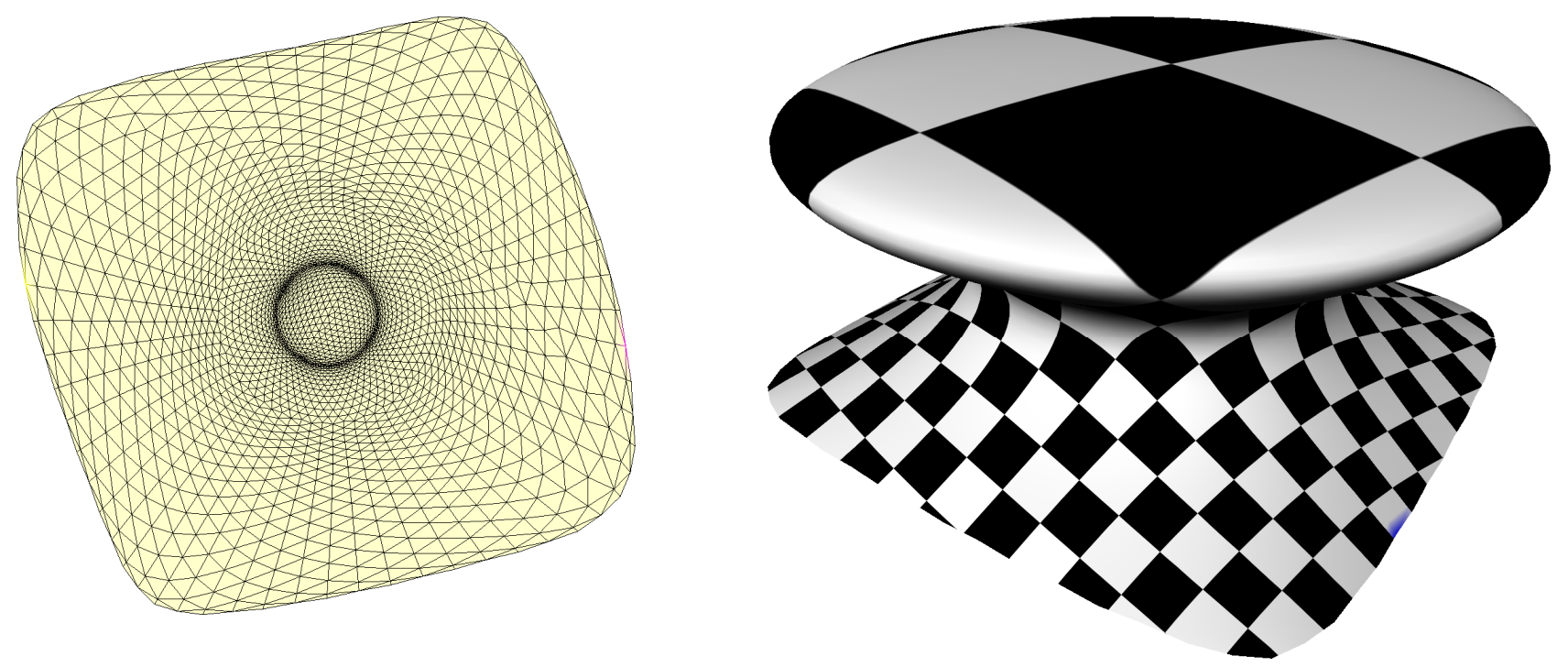


\section{Example}

\#include <CGAL/Cartesian.h>

\#include <CGAL/Polyhedron_3.h>

\#include <CGAL/Parameterization_polyhedron_adaptor_3.h>

\#include <CGAL/parameterize.h>

Polyhedron mesh;

Mesh_adaptor_polyhedron mesh_adaptor(\&mesh);

CGAL : :parameterize (\&mesh_adaptor);

Point_2 uv = mesh_adaptor.info(he) $\rightarrow$ uv ();
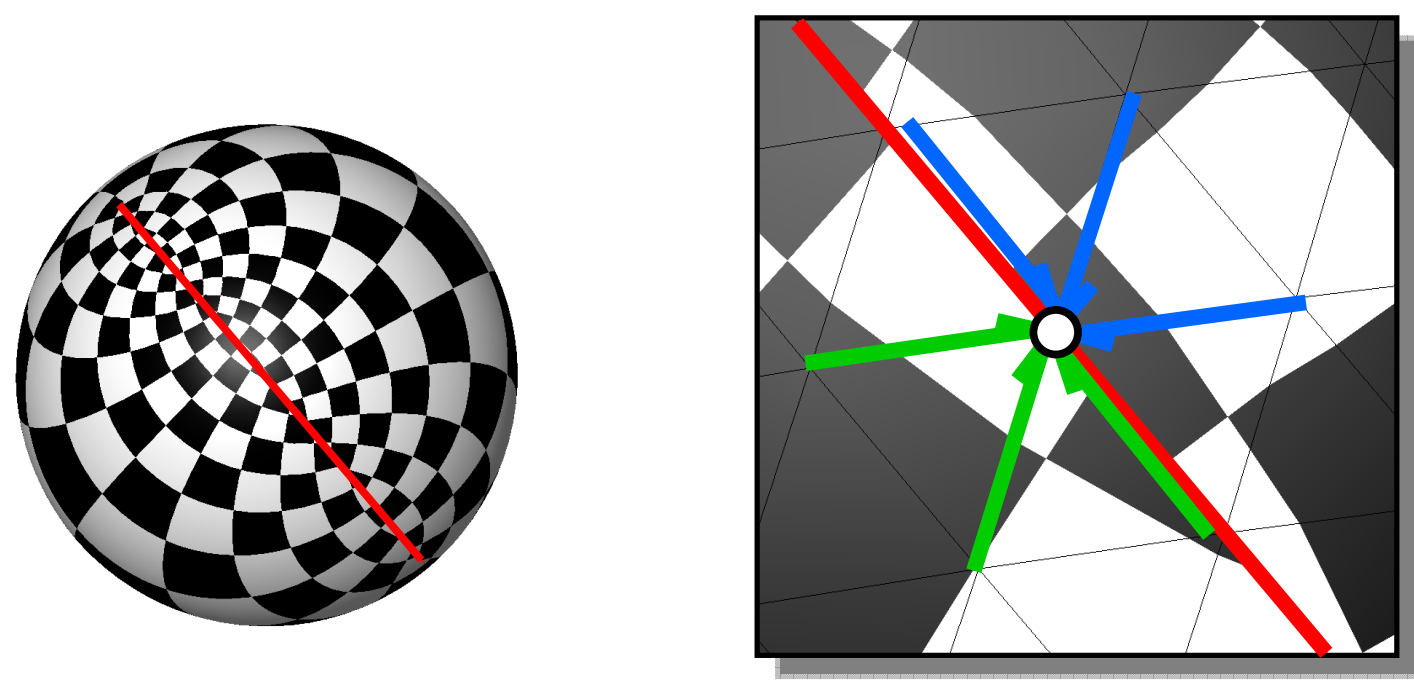


\section{Parameterization}

User-provided cut graph for closed or high genus surfaces.
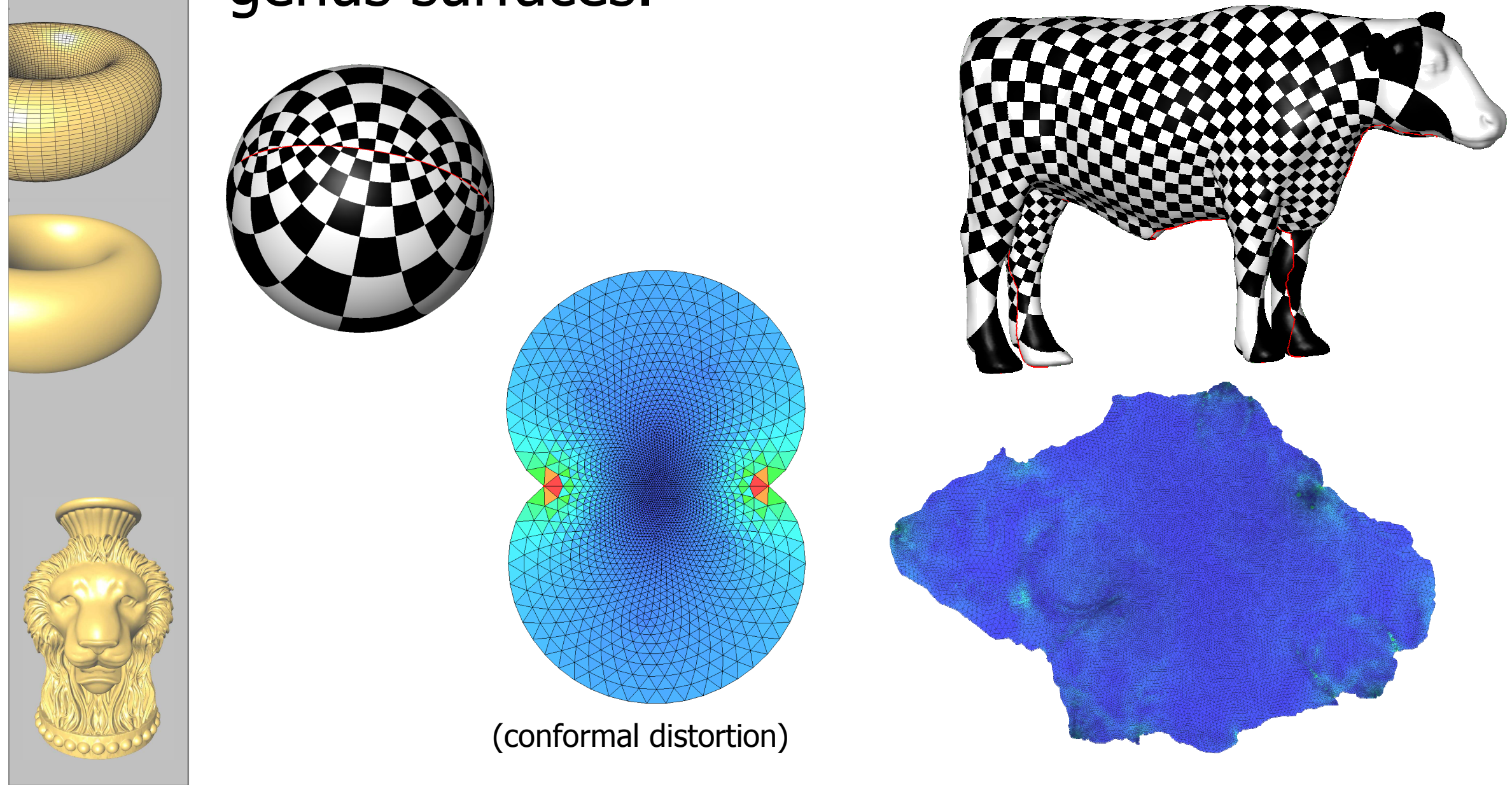


\section{Parameterization}
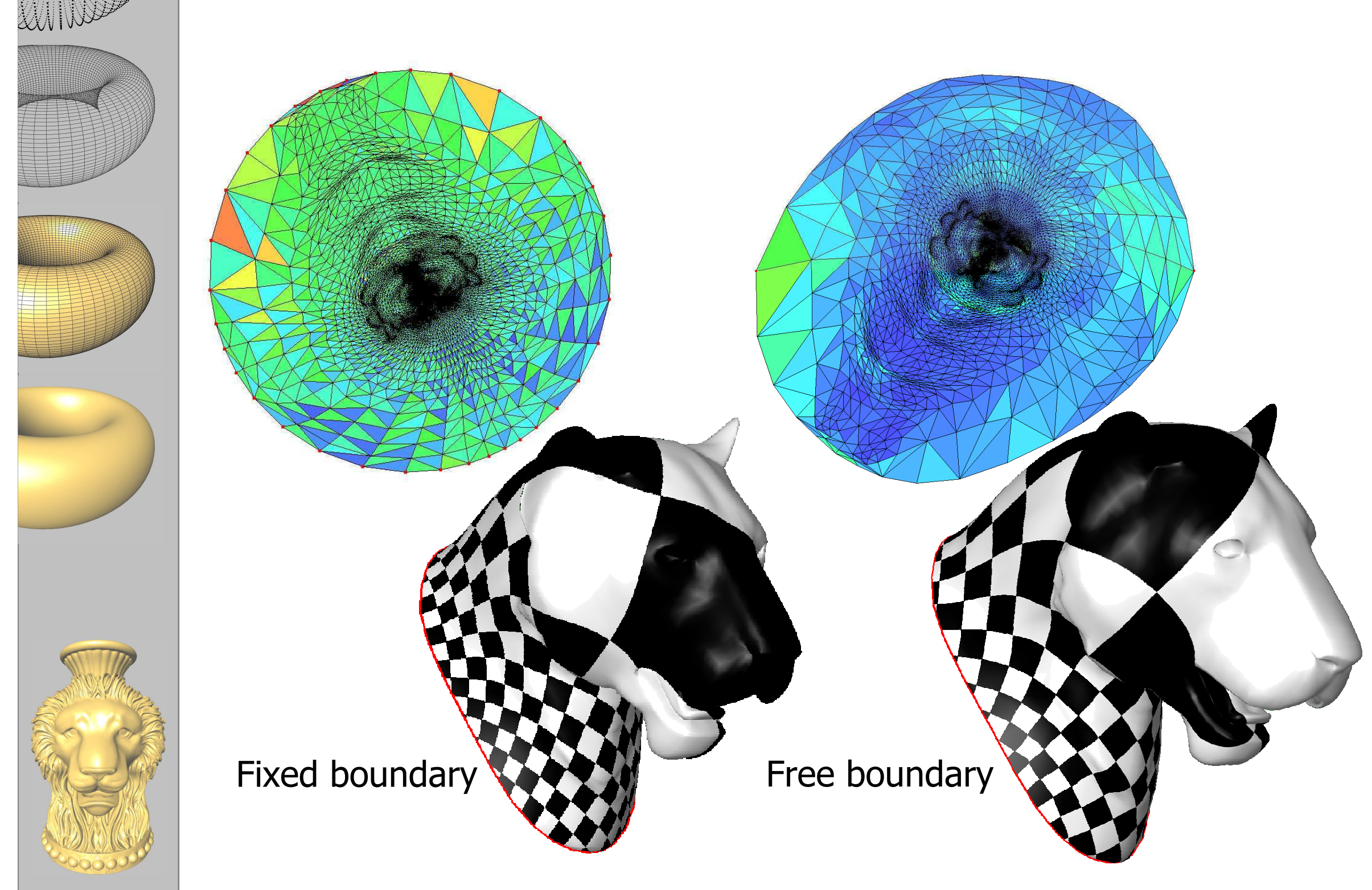


\section{Subdivision}

Designed to work on CGAL polyhedron

- Catmull-Clark

- Loop

- Doo-Sabin

- Sqrt3

- ...
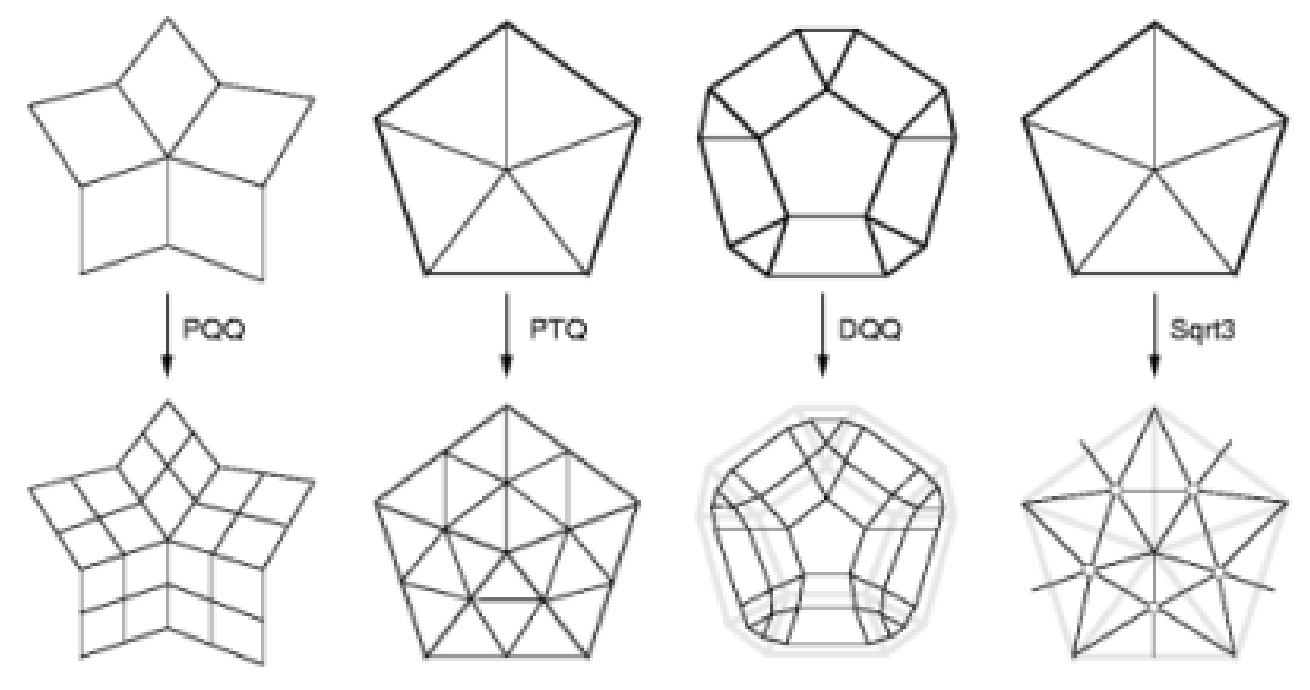


\section{Example}

\#include <CGAL/Cartesian.h>

\#include <CGAL/Polyhedron_3.h>

\#include <CGAL/Subdivision_method_3.h>

typedef CGAL: :Cartesian<double>

Kernel;

typedef CGAL: :Polyhedron_3<Kernel>

Polyhedron;

using CGAL: :Subdivision_method_3;

Polyhedron polyhedron;

int subdivision_depth $=3$;

Catmullclark_subdivision (polyhedron, subdivision_depth) ;
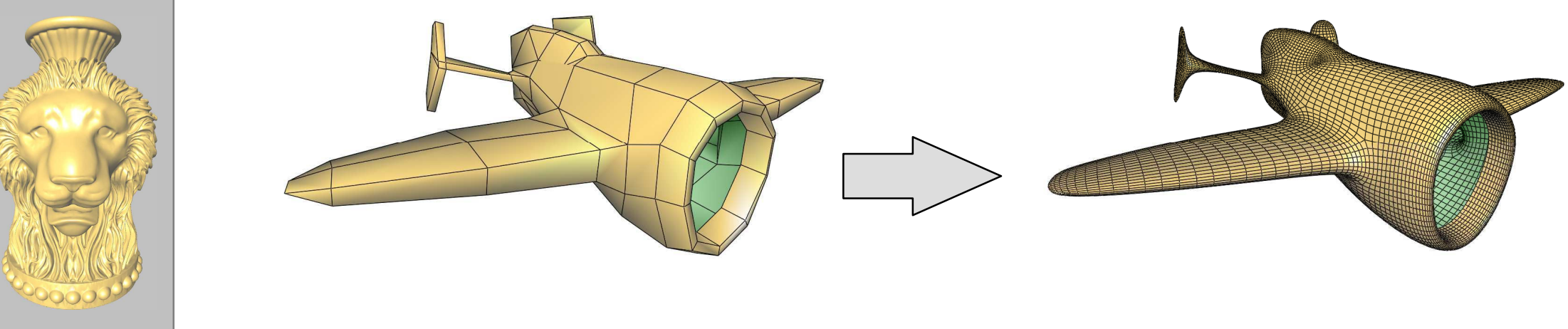


\section{Principal Component Analysis}

Linear least squares fitting on sets of 3D kernel objects:

- points

for triangle

- triangles $\}$ meshes

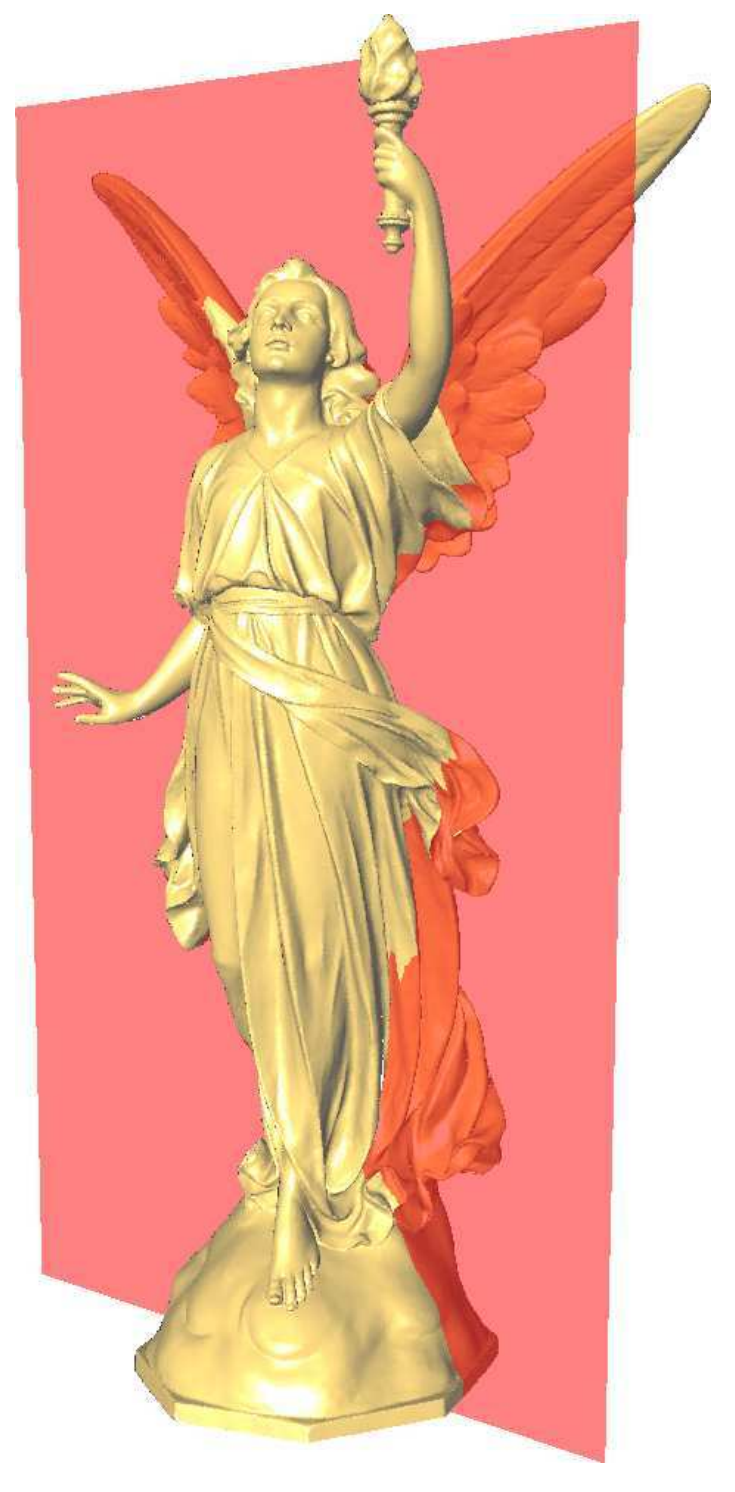




\section{Example}

\#include <CGAL/linear_least_squares_fitting_3.h>

// more \#includes and typedefs

Polyhedron mesh;

std: : list<Triangle_3> triangles;

Polyhedron: : Facet_iterator $f$;

for ( $f=$ mesh.facets_begin();

f ! = mesh.facets_end ();

$++f)\{$

const Point\& $a=$ f->halfedge ()->vertex ()->point ();

const Point\& $b=f->$ halfedge ()$->$ next ()$->\operatorname{vertex}() \rightarrow$ point ( $)$;

const Point\& $c=f->$ halfedge ()$->$ prev ()$->$ vertex ()$\rightarrow$ point () ;

triangles.push_back (Triangle_3(a,b,c)) ;

\}

Plane_3 plane;

CGAL: :linear_least_squares_fitting_3( triangles.begin(), triangles.end (),

plane,

CGAL : :PCA_dimension_2_tag() ); 


\section{Same for Boost freaks $\odot$}

\#include <CGAL/linear_least_squares_fitting_3.h> \#include <boost/iterator/transform_iterator.hpp>

Polyhedron mesh;

class ToTriangle \{

Triangle_3 operator (Polyhedron: :FacetIterator f) \{ return Triangle_3 ( $f$->halfedge () ->vertex () $\rightarrow$ point ();

f->halfedge () ->next () ->vertex () ->point () ;

\}

f->halfedge () ->prev () ->vertex ()->point () );

\} ;

Plane_3 plane;

CGAL : : linear_least_squares_fitting_3 (

boost: :make_transform_iterator(mesh.facets_begin (), ToTriangle ()),

boost : :make_transform_iterator(mesh.facets_end (), ToTriangle ()),

(II) plane, CGAL: :PCA_dimension_2_tag()); 


\section{Fitting Points vs Triangles}

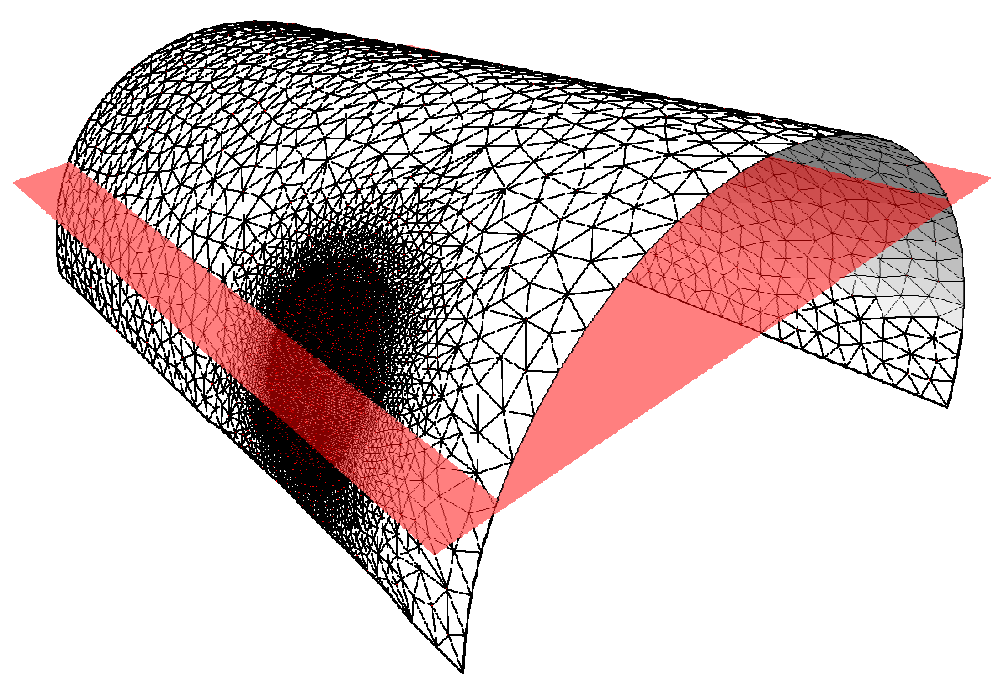

fit points

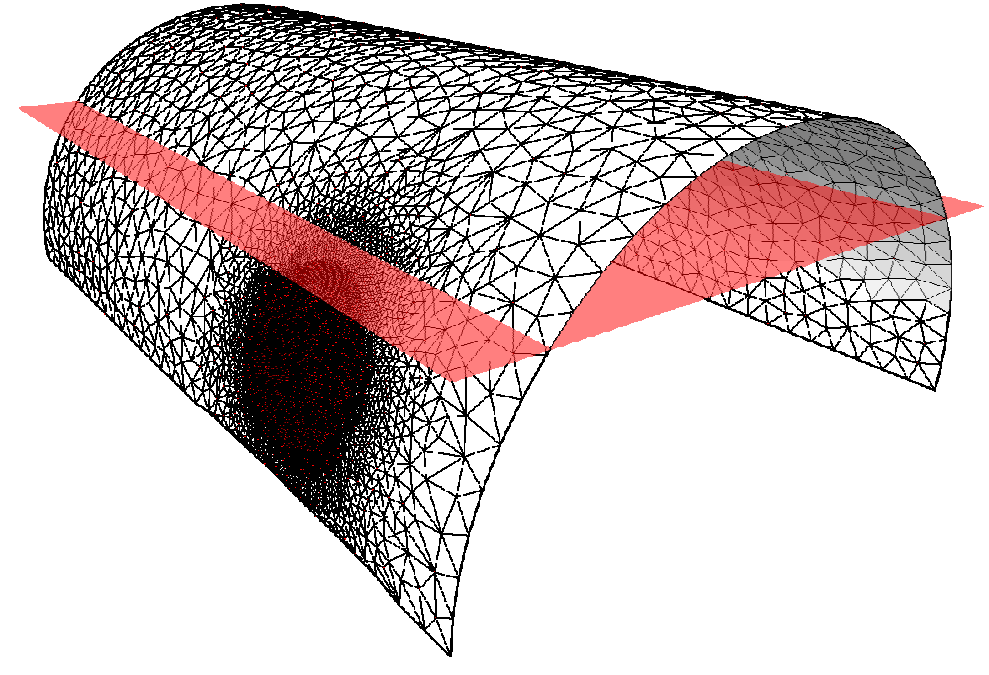

fit triangles 


\section{Estimation of Curvatures}

- Estimates general differential properties (Monge form) on point sets.

- Through polynomial (d-jet) fitting
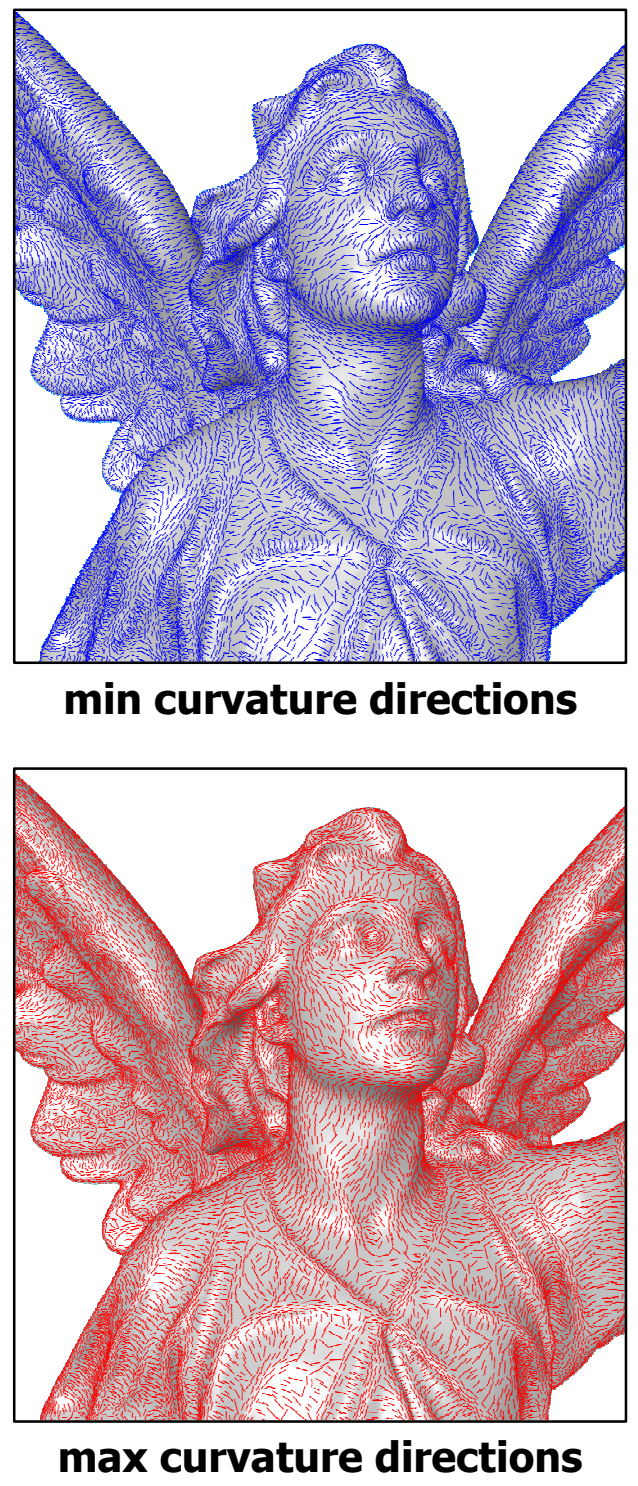


\section{Example}

\#include <CGAL/Monge_via_jet_fitting.h>

typedef CGAL::Cartesian<double> Kernel;

typedef CGAL: :Monge_via_jet_fitting<Kernel> Monge_fit;

typedef Monge_fit::Monge_form Monge_form;

Monge_fit monge_fit;

Monge_form monge_form =

monge_fit ( points.begin(),

points.end (),

dim_fitting, dim_monge);

Vector_3 kmin = monge_form.minimal_principal_direction ();

Vector_3 $\mathbf{k m a x}=$ monge_form.maximal_principal_direction ();

Vector_3 normal = monge_form.normal_direction (); 


\section{Extraction of Ridges}

Ridge: curve along which one of the principal curvatures has an extremum along its curvature line.

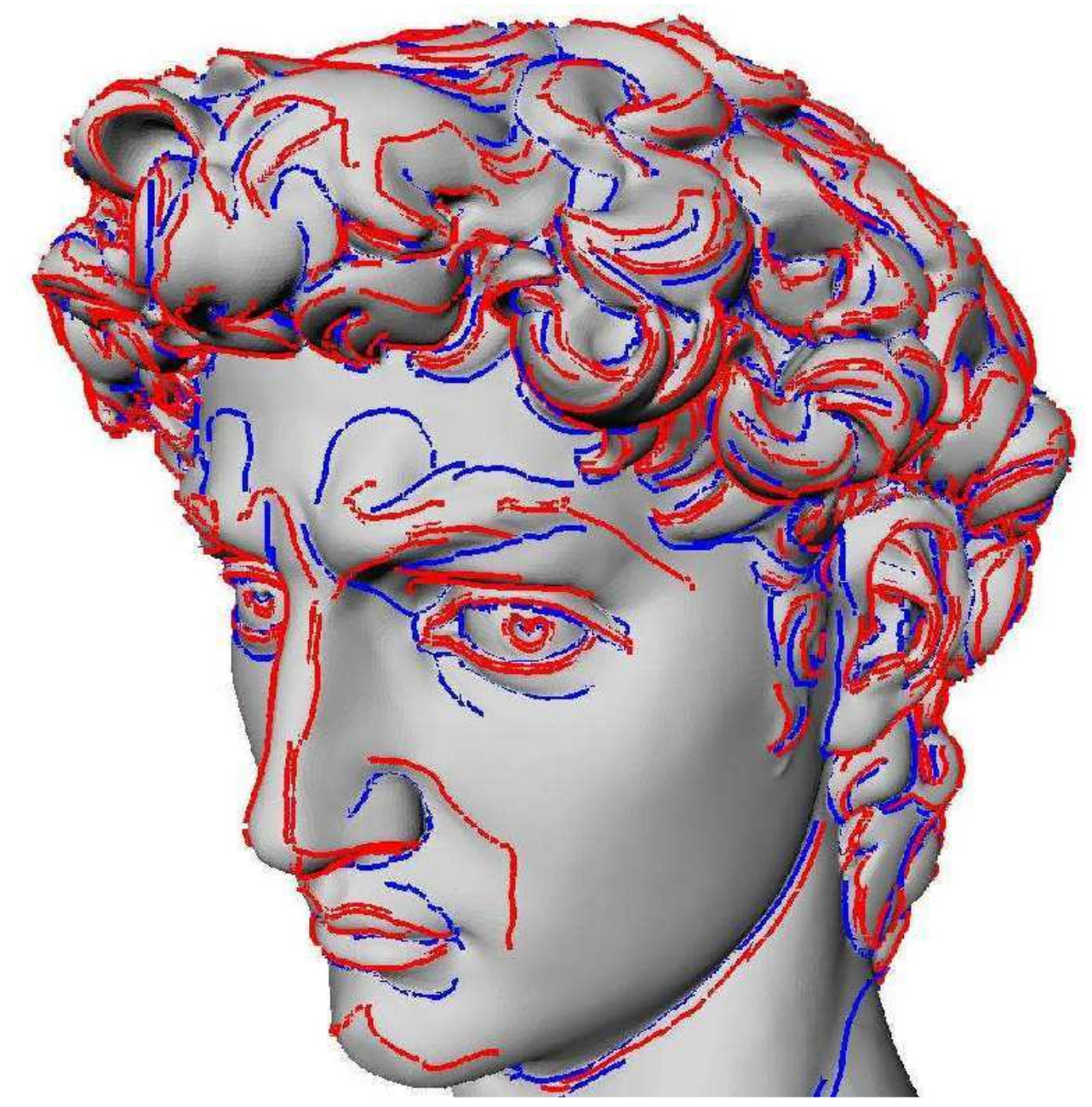




\section{Simplification}

Implementation of [LindstromTurk] volume-preserving method. BGL-style allows simplification of any model of EmbeddedGraph
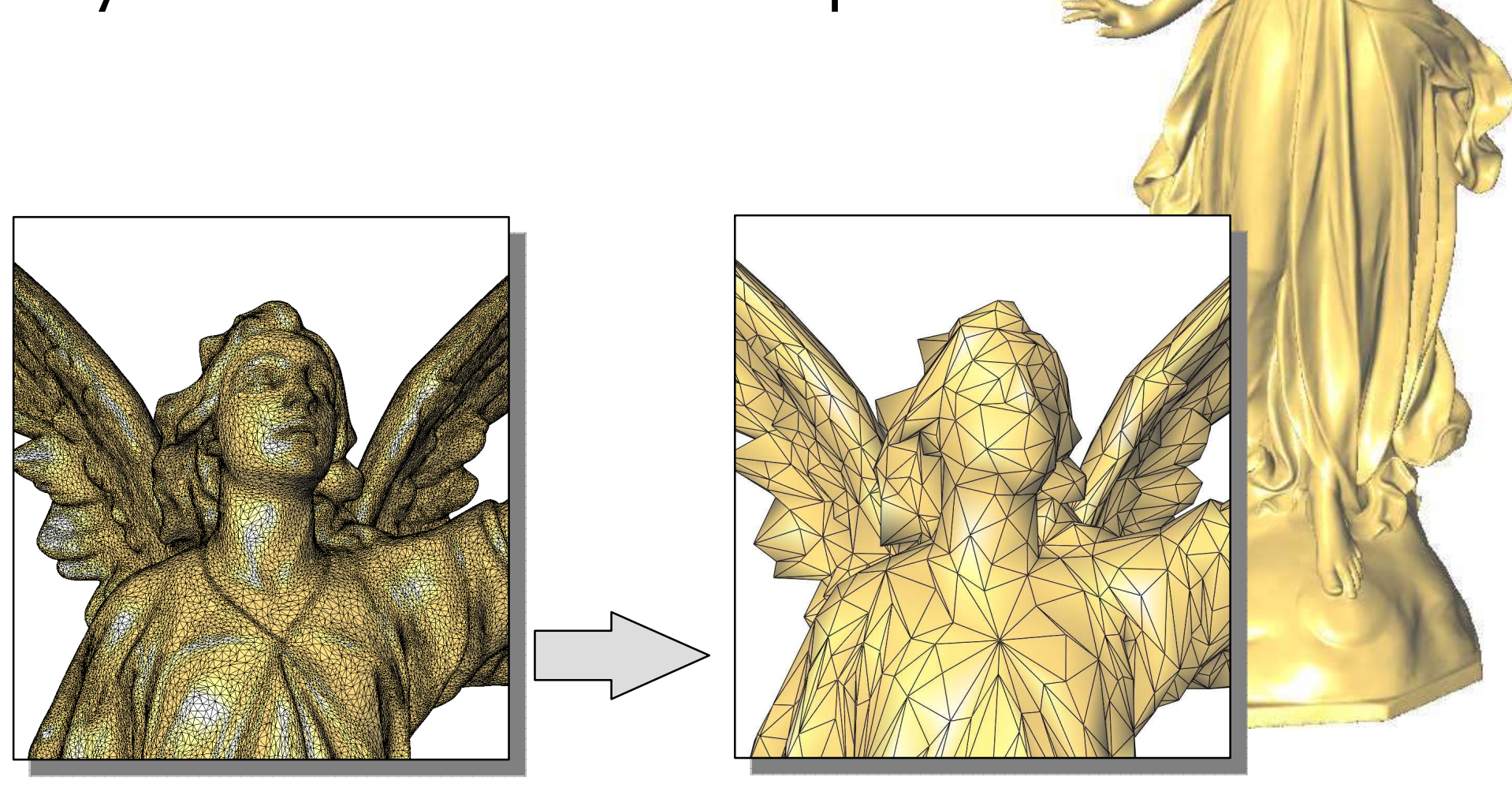


\section{Simplification: Example}

\#include <CGAL/Simple_cartesian.h>

\#include <CGAL/Polyhedron_3.h>

\#include <CGAL/Surface_mesh_simplification/HalfedgeGraph_Polyhedron_3.h>

\#include <CGAL/Surface_mesh_simplification/edge_collapse.h>

\#include

<CGAI/Surface_mesh_simplification/Policies/Edge_collapse/Count_stop_predicate.h $>$

typedef CGAL: :Simple_cartesian<double> Kernel;

typedef CGAL: :Polyhedron_3<Kernel> Mesh;

namespace SMS = CGAL::Surface_mesh_simplification ;

Mesh mesh;

SMS : : Count_stop_predicate< Mesh > stop(1000); // target \# edges

SMS: :edge_collapse(mesh, stop,

CGAL : :vertex_index_map (boost : : get (CGAL : :vertex_external_index, mesh) )

.edge_index_map (boost : : get (CGAL: :edge_external_index, mesh)) ); 


\section{Summary and Outlook}

- The halfedge data structure and the polyhedron are highly flexible

- CGAL provides algorithms for geometric modeling and geometry processing

- Polyhedral surface as output of surface mesh generation algorithms (Part IV) 


\section{Under Development}

- BGL-ization of existing CGAL algorithms 


\section{Under Development}

- BGL-ization of existing CGAL algorithms

- Remeshing
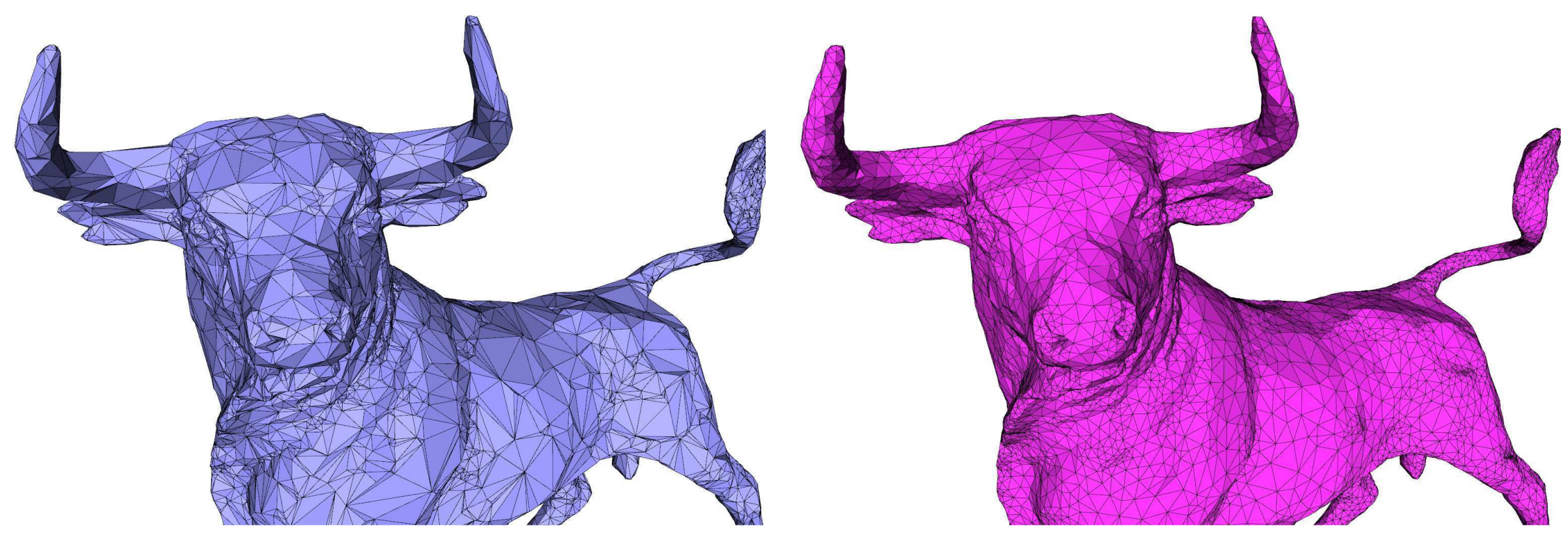
Questions? 


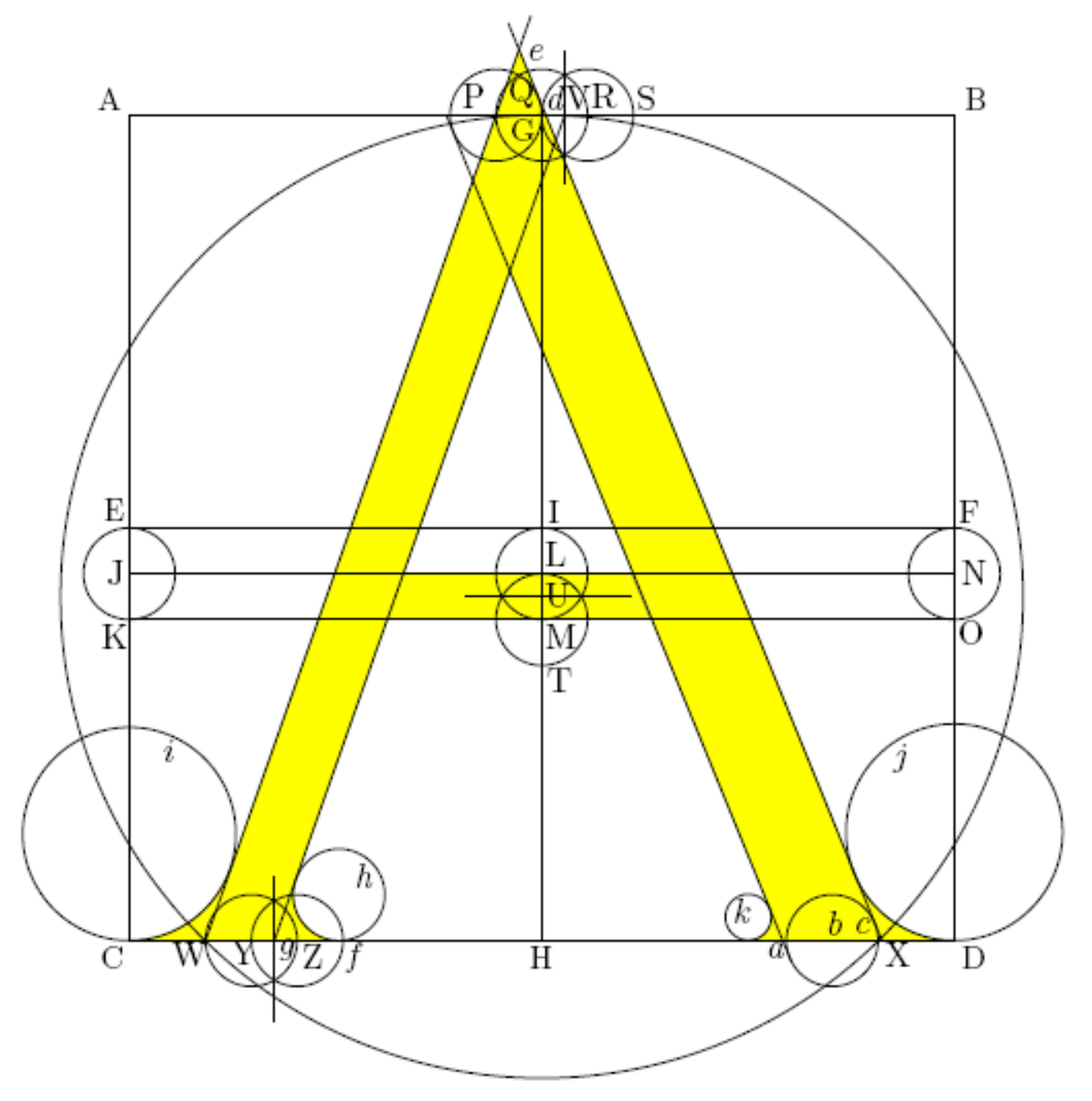

\title{
Arrangements
}

\author{
Efi Fogel
}

Tel Aviv University 


\section{Outline}

- Arrangements

- Algorithms based on Arrangements

- Boolean Set Operations

- Minkowski Sums and Polygon Offset

- Envelopes

- Arrangements on Surfaces 


\section{Arrangement Definition}

Given a collection of curves on a surface, the arrangement is the partition of the surface into vertices, edges and faces induced by the curves

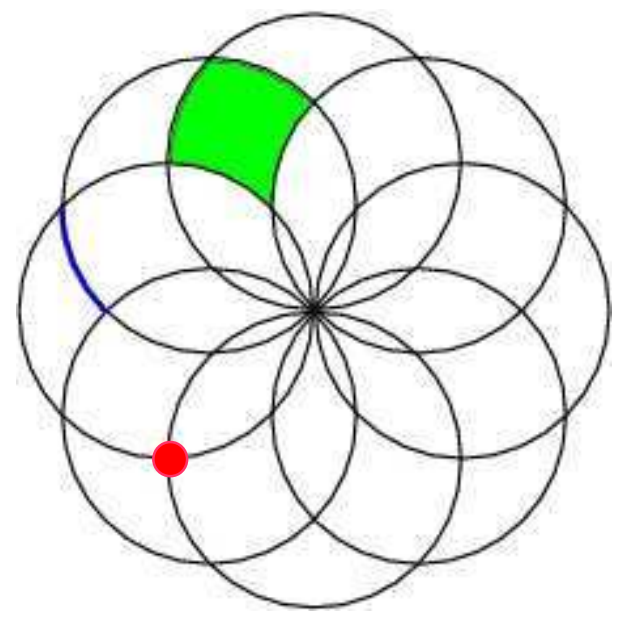

An arrangement of circles in the plane

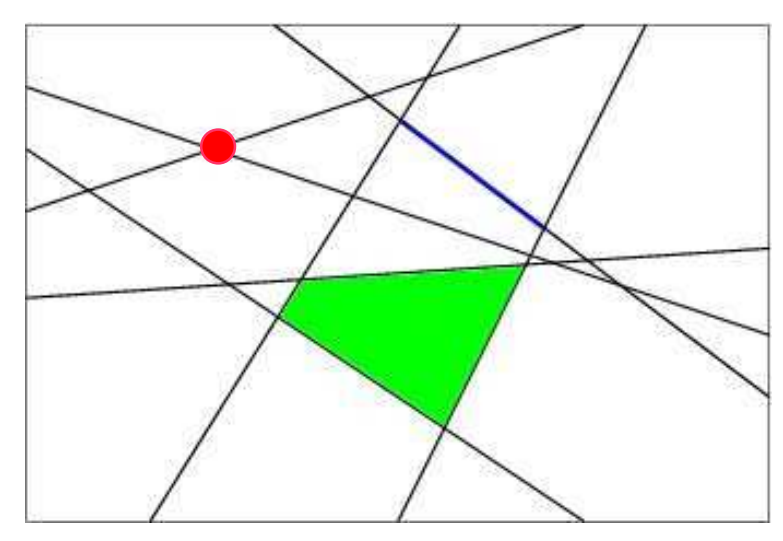

An arrangement of lines in the plane

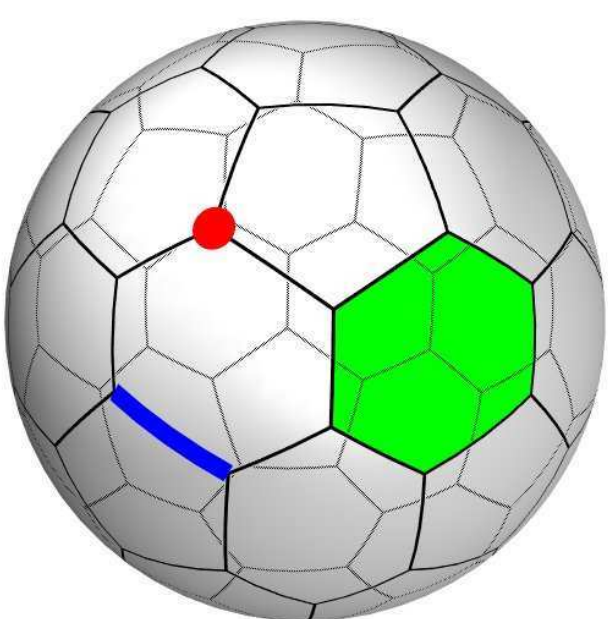

An arrangement of geodesic arcs on the sphere 


\section{Code: A Simple Arrangement}

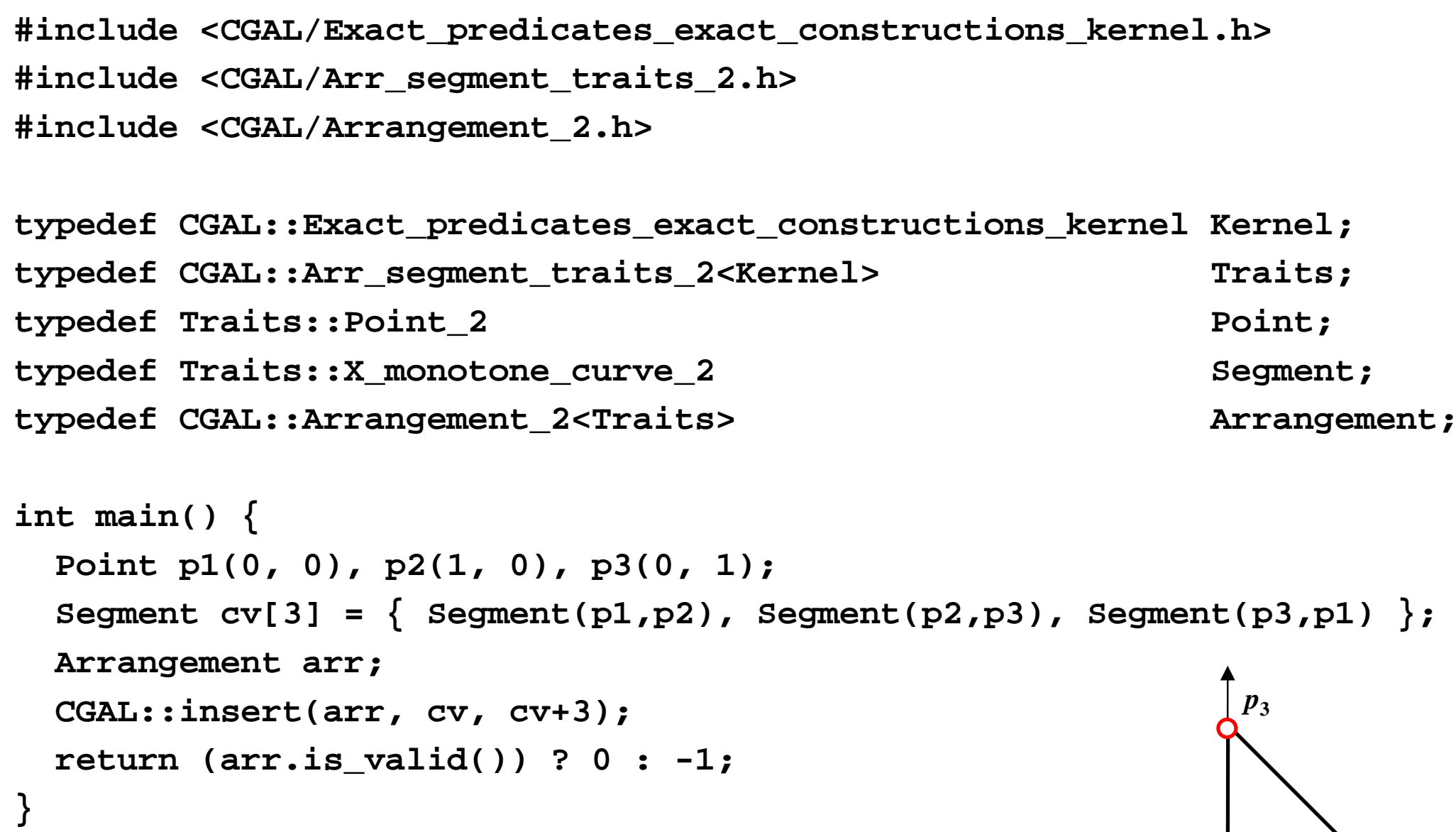




\section{CGAL::Arrangement_2}

- Constructs, maintains, modifies, traverses, queries, and presents subdivisions of the plane

- Robust and exact

- All inputs are handled correctly (including degenerate)

- Exact number types are used to achieve exact results

- Efficient

- Generic

- Easy to interface, extend, and adapt

- Notification mechanism for change propagation

- Modular

- Geometric and topological aspects are separated 


\section{Geometric Trailts}

- Define the family of curves

- Aggregate geometric types and operations over the types

- Compare two points

- Determine the relative position of a point and an $x$-monotone curve

- Determine the relative position of two $x$ monotone curves to the left (right) of a point

- Subdivide a curve into x-monotone curves

- Find all intersections of two xmonotone curves

\section{point}




\section{Geometric Traits}

- Define the family of curves

- Aggregate geometric types and operations over the types

- Compare two points

- Determine the relative position of a point and an $x$-monotone curve

- Determine the relative position of two $x$ monotone curves to the left (right) of a point

- Subdivide a curve into x-monotone curves

- Find all intersections of two xmonotone curves

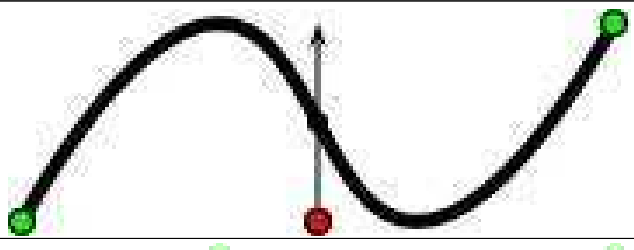




\section{Geometric Traits}

- Define the family of curves

- Aggregate geometric types and operations over the types

- Compare two points

- Determine the relative position of a point and an $x$-monotone curve

- Determine the relative position of two $x$ monotone curves to the left (right) of a point

- Subdivide a curve into x-monotone curves

- Find all intersections of two xmonotone curves

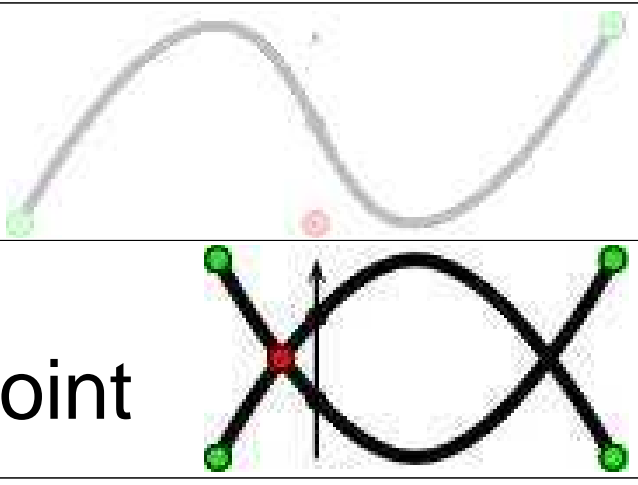




\section{Geometric Trails}

- Define the family of curves

- Aggregate geometric types and operations over the types

- Compare two points

- Determine the relative position of a point and an $x$-monotone curve

- Determine the relative position of two $x$ monotone curves to the left (right) of a point

- Subdivide a curve into x-monotone curves

- Find all intersections of two $\mathrm{x}$ monotone curves

\section{point}




\section{Geometric Traits}

- Define the family of curves

- Aggregate geometric types and operations over the types

- Compare two points

- Determine the relative position of a point and an $x$-monotone curve

- Determine the relative position of two $x$ monotone curves to the left (right) of a point

- Subdivide a curve into x-monotone curves

- Find all intersections of two $x-$ monotone curves

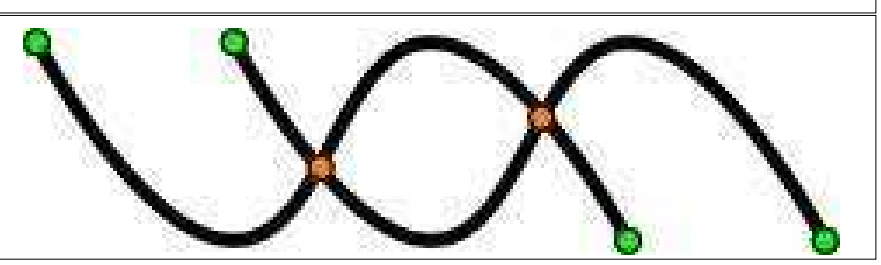




\section{Arrangement Trailts Classes}

\begin{tabular}{|c|c|c|c|c|c|c|}
\hline Curve Family & Degree & Surface & Boundness & Arithmetic & Attribute & \\
\hline linear segments & 1 & plane & bounded & rational & $\begin{array}{l}\text { caching } \\
\text { noncaching }\end{array}$ & y \\
\hline linear segments, rays, lines & 1 & plane & unbounded & rational & & 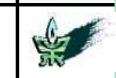 \\
\hline piecewise linear curves & $\infty$ & plane & bounded & rational & $\begin{array}{l}\text { caching } \\
\text { noncaching }\end{array}$ & ty \\
\hline circular arcs, linear segments & $\leq 2$ & plane & bounded & rational & CK & 要 \\
\hline algebraic curves & $\leq 2$ & plane & $\begin{array}{l}\text { Bounded } \\
\text { unbounded }\end{array}$ & algebraic & $\begin{array}{l}\text { CORE } \\
\text { CKvA_2 }\end{array}$ & י \\
\hline quadric projections & $\leq 2$ & plane & unbounded & algebraic & & II \\
\hline algebraic curves & $\leq 3$ & plane & unbounded & algebraic & & II \\
\hline algebraic curves & $\leq \mathrm{n}$ & plane & unbounded & algebraic & & \\
\hline planar Bézier curves & $\leq \mathrm{n}$ & plane & unbounded & algebraic & & 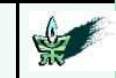 \\
\hline univariate polynomials & $\leq \mathrm{n}$ & plane & unbounded & algebraic & RS & R \\
\hline rational function arcs & $\leq \mathrm{n}$ & plane & unbounded & algebraic & & 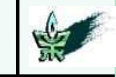 \\
\hline geodesic arcs on sphere & $\leq 2$ & sphere & bounded & rational & & 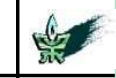 \\
\hline quadric intersection arcs & $\leq 2$ & quadric & unbounded & algebraic & & '"' \\
\hline dupin cyclide intersection. arcs & $\leq 2$ & dupin cyclides & bounded & algebraic & & יו"י \\
\hline
\end{tabular}




\section{Enrich Vertices, Edges, Faces}

template <typename Traits, typename Dcel = Arr_default_dcel<Traits_> > class Arrangement_2

\};

enum Color \{BLUE, RED, WHITE\};

typedef CGAL::Arr_segment_traits_2<Kernel> Traits;

typedef Traits: :Point_2 Point;

typedef Traits: :x_monotone_curve_2 Segment;

typedef CGAL: :Arr_extended_dcel<Traits, Color, Color, Color> Dcel;

typedef CGAL: :Arrangement_2<Traits, Dcel> Arrangement; 


\section{Point Location}

Given a subdivision $A$ of the space into cells and a query point $q$, find the cell of $A$ containing $q$
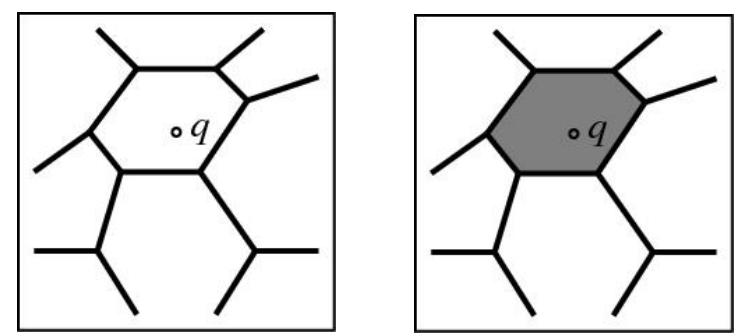

- Fast query processing

- Reasonably fast preprocessing

- Small space data structure

\begin{tabular}{l|l|l|l|l} 
& Naive & \multicolumn{1}{c|}{ Walk } & \multicolumn{1}{c|}{ RIC } & Landmarks \\
\hline Preprocessing time & none & none & O(n log $\mathrm{n})$ & $\mathrm{O}(\mathrm{k} \log \mathrm{k})$ \\
\hline Memory space & none & none & $\mathrm{O}(\mathrm{n})$ & $\mathrm{O}(\mathrm{k})$ \\
\hline Query time & bad & reasonable & good & good \\
\hline Applicability & all & limited & limited & limited
\end{tabular}

Walk - Walk along a line

RIC - Random Incremental Construction based on trapezoidal decomposition

$k$ - number of landmarks 


\section{Code: Point Location}

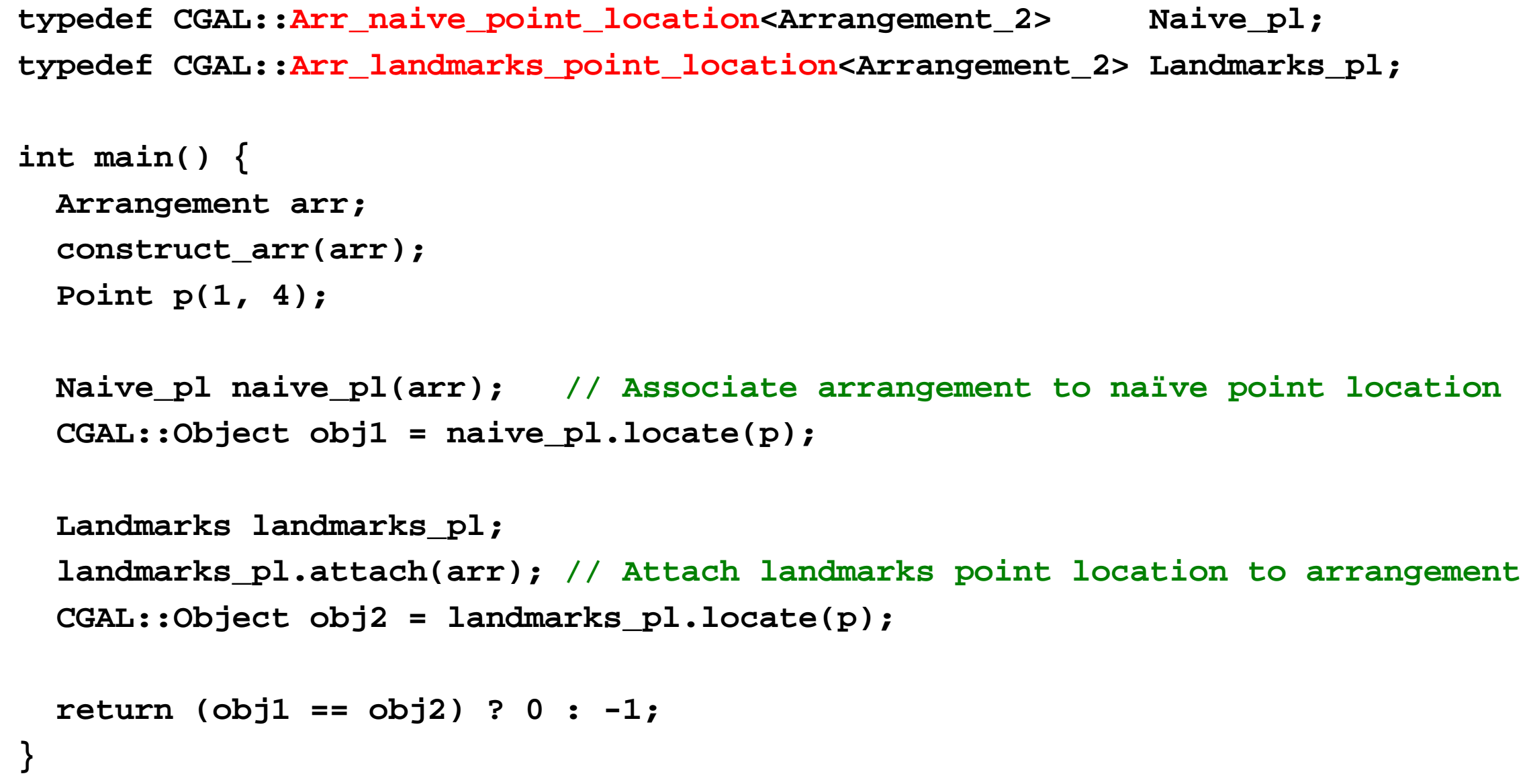


Boolean Set Operations

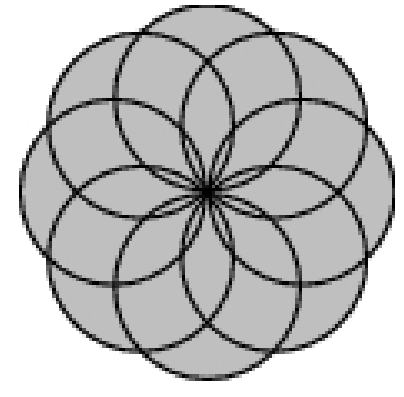

Union
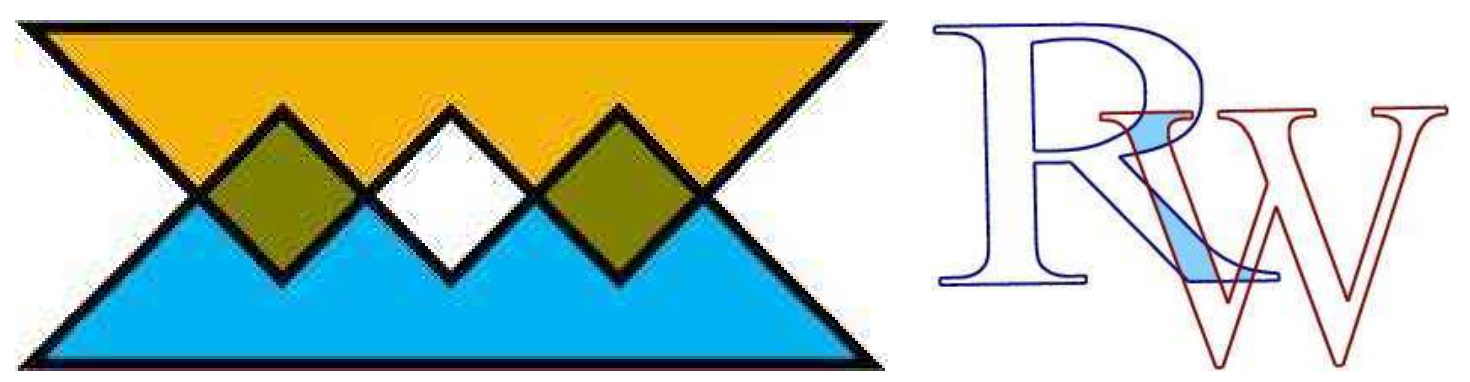

Intersection

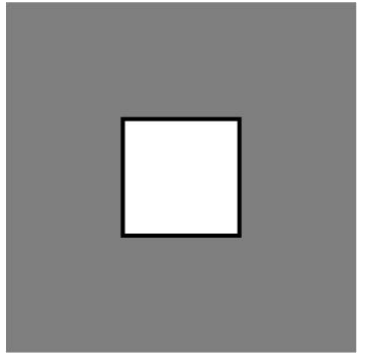

Complement

For two point sets $P$ and $Q$ and a point $r$ :

\begin{tabular}{ll}
\hline Complement & $R=\bar{P}$ \\
\hline Union & $R=P \cup Q$ \\
\hline Intersection & $R=P \cap Q \quad=\bar{P} \cup \bar{Q}$ \\
\hline Difference & $R=P \backslash Q \quad=P \cap \bar{Q}$ \\
\hline Symmetric Difference & $R=(P \backslash Q) \cup(Q \backslash P)$ \\
\hline Intersection predicate & $P \cap Q \neq \varnothing \quad$ Overlapping cell(s) are not explicitly computed \\
\hline Containment predicate & $r \in P$ \\
\hline Interior, Boundary, Closure & \\
\hline Regularization & $R=$ closure(interior $(P))$ \\
\hline
\end{tabular}




\section{Code: Simple BOPs}

int main () \{

Polygon p, q;

p.push_back (Point $(0,0))$; p.push_back (Point $(2,0)$ ) ; p.push_back (Point $(1,1))$; p.push_back (Point $(0,2))$; q.push_back (Point $(1,1))$; q.push_back (Point $(3,1)$ ); q.push_back (Point $(2,2)) ;$ q.push_back (Point $(1,3)$ ) ;

Polygon_with_holes comp_p, comp_q;

CGAL : : complement ( $p$, comp_p) ;

CGAL : : complement ( $q$, comp_q) ;

Polygon_with_holes a;

CGAL : : join (comp_p, comp_q, a) ;

std: : list<Polygon_with_holes> 11, 12;

CGAL : : complement (a, std: :back_inserter(11)) ;

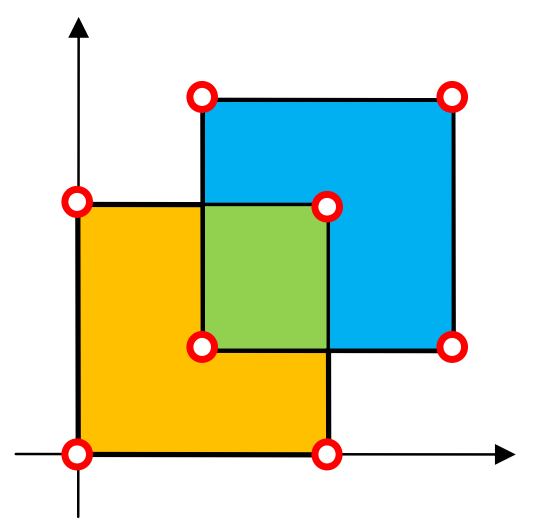

CGAL: : intersection ( $p, q, 12)$;

return std: : compare $(11,12)$; 


\section{CGAL::Boolean_set_operation_2}

- Supports

- regularized Boolean set-operations

- intersection predicates

- point containment predicates

- Operands and results are regularized point sets bounded by $x$-monotone curves referred to as general polygons

- General polygons may have holes

- Extremely efficient aggregated operations

- Based on the Arrangement_2 and Polygon_2 packages 


\section{Minkowski Sum in $\mathbf{R}^{\mathrm{d}}$}

$P$ and $Q$ are 2 polytopes in $R^{d}$

$P \oplus Q=\{p+q \mid p \in P, q \in Q\} \quad$ Minkowski sum
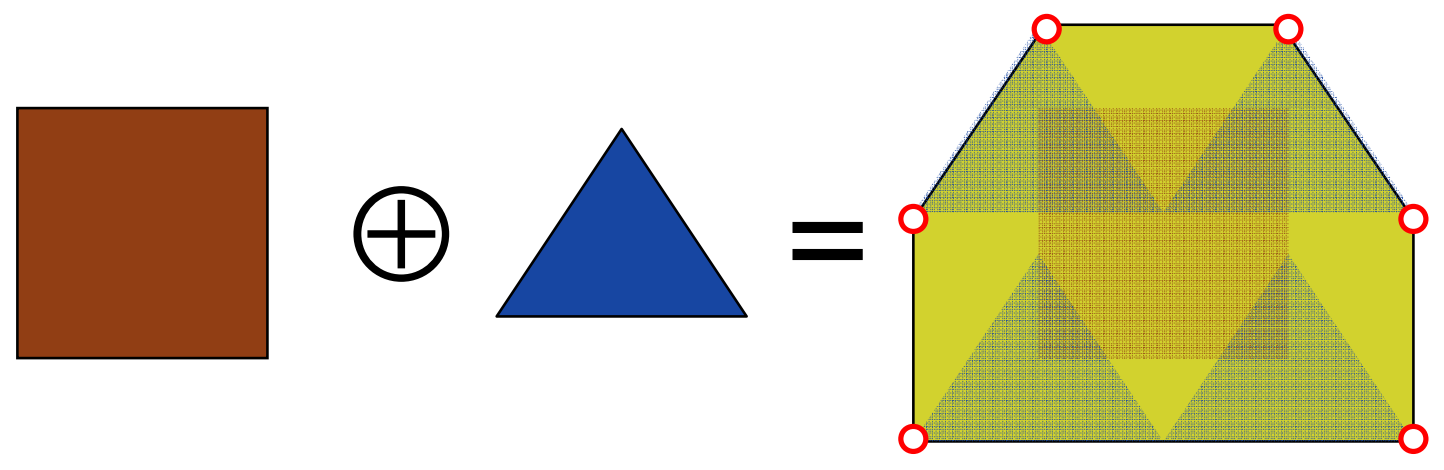

$P \cap Q \neq \varnothing \Leftrightarrow$ Origin $\in P \oplus(-Q) \quad$ collision
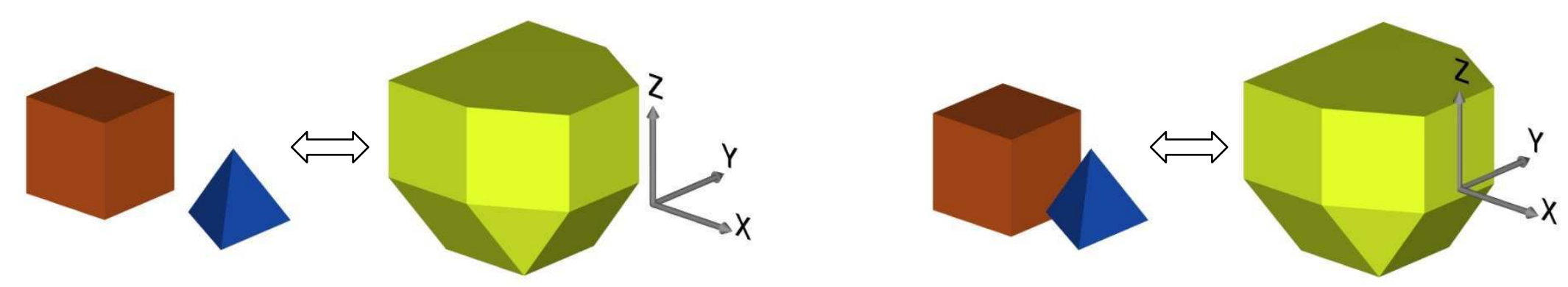


\section{CGAL::Minkowski_sum_2}

- Based on the Arrangement_2, Polygon_2, and Partition_2 packages

- Supports Minkowski sums of two simple polygons

- Implemented using either decomposition or convolution

- Exact

- Interoperable with Boolean_set_operations_2, e.g., Compute the union of offset polygons

- Supports Minkowski sums of a simple polygon and a disc (polygon offsetting)

- Offers either an exact computation or a conservative approximation scheme

- Disk radius can be negative (inner offset) 


\section{Motion Planning}

- The input robot and the obstacle are non-convex

- Exploits the convolution method

- The output sum contains four holes, isolated points, and antennas
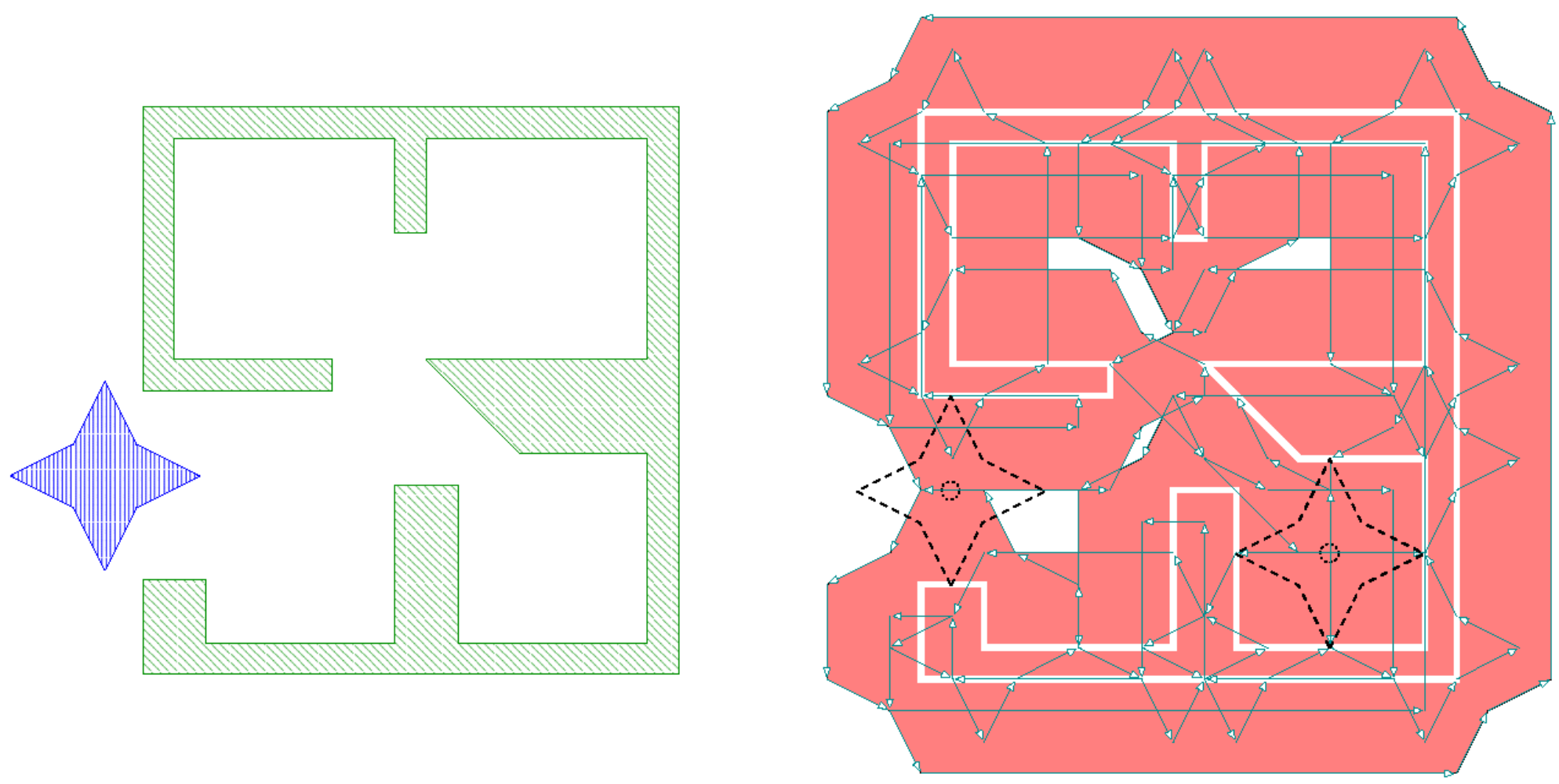


\section{Envelopes in $\mathrm{R}^{3}$}

The lower envelope of a set of $x y$-monotone surfaces $S=$ $\left\{S_{1}, S_{2}, \ldots, S_{n}\right\}$, is the point-wise minimum of all surfaces The minimization diagram of $S$ is an arrangement

- The identity of the surfaces that induce the lower envelope over a specific cell (vertex, edge, face) of the arrangement is the same
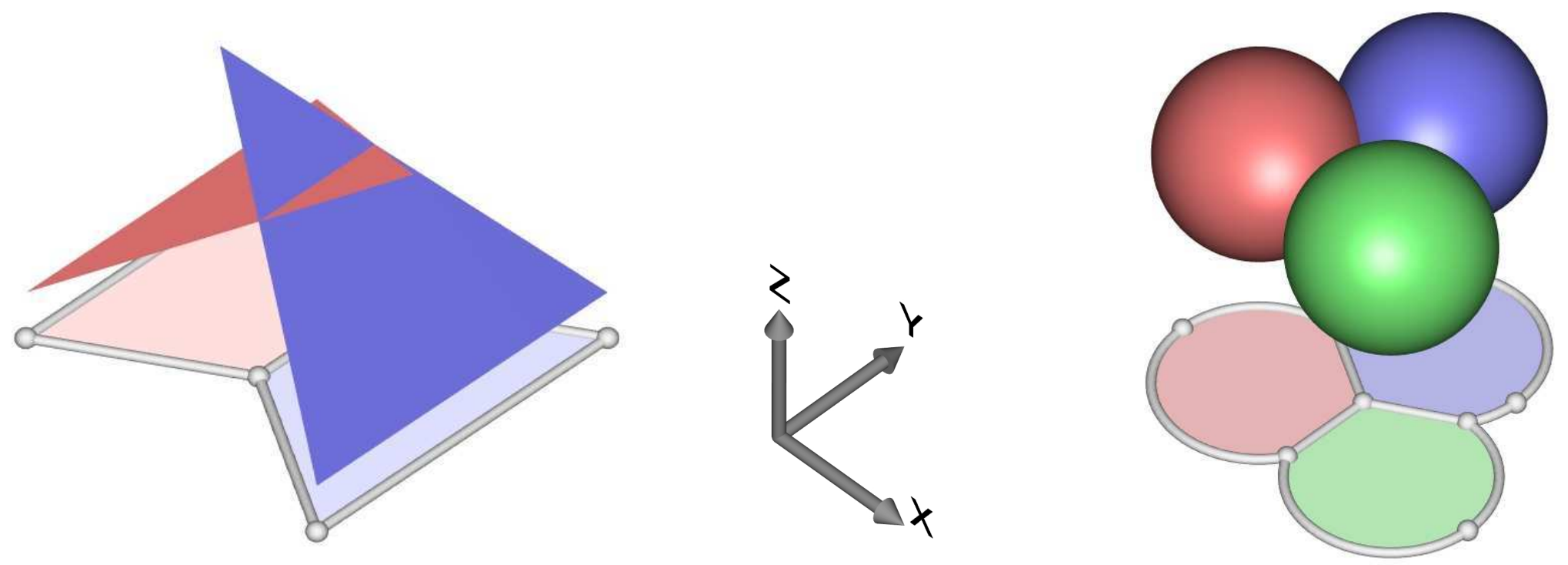


\section{Code: Lower Envelope}

\#include <CGAL/Exact_predicates_exact_constructions_kernel.h>

\#include <CGAL/Env_triangle_traits_3.h>

\#include <CGAL/Env_surface_data_traits_3.h>

\#include <CGAL/envelope_3.h>

typedef CGAL: :Exact_predicates_exact_constructions_kernel Kernel;

typedef CGAL: :Env_triangle_traits_3<Kernel> Traits;

typedef Traits: :Point_3 Point;

typedef Traits::Surface_3 Tri;

enum Color \{RED, BLUE\};

typedef CGAL: :Env_surface_data_traits_3<Traits, Color> Data_traits;

typedef Data_traits: :Surface_3

typedef CGAL: :Envelope_diagram_2<Data_traits> Arrangement;

int main () \{

Point $\mathrm{p} 1(0,0,1)$, p2 $(0,6,1)$, p3 $(5,3,5), \mathrm{p} 4(6,0,1), \mathrm{p} 5(6,6,1), \mathrm{p} 6(1,3,5)$;

Tri tris []$=\{\operatorname{Dtri}(\operatorname{Tri}(\mathrm{p} 1, \mathrm{p} 2, \mathrm{p} 3), \operatorname{RED}), \operatorname{Dtri}(\operatorname{Tri}(\mathrm{p} 4, \mathrm{p} 5, \mathrm{p} 6), \mathrm{BLUE})\}$;

Arrangement arr;

CGAL : : lower_envelope_3 (tris, tris+2, arr);

return (arr.is_valid()) ? 0 : -1 ; 


\section{CGAL::Envelope_3}

- Constructs lower and upper envelopes of surfaces

\begin{tabular}{l|l|l} 
Surface Family & Class Name & \\
\hline triangles & Env_triangle_traits_3 & \\
\hline spheres & Env_sphere_traits_3 & \\
\hline planes and half planes & Env_plane_traits_3 & \\
\hline quadrics & Env_quadric_traits_3 & '.'p:
\end{tabular}

- Based on the Arrangement_2 package

- Exploits

- Overlay computation (using the sweep line framework)

- Isolated points

- Zone traversal 


\section{Lower Envelopes}
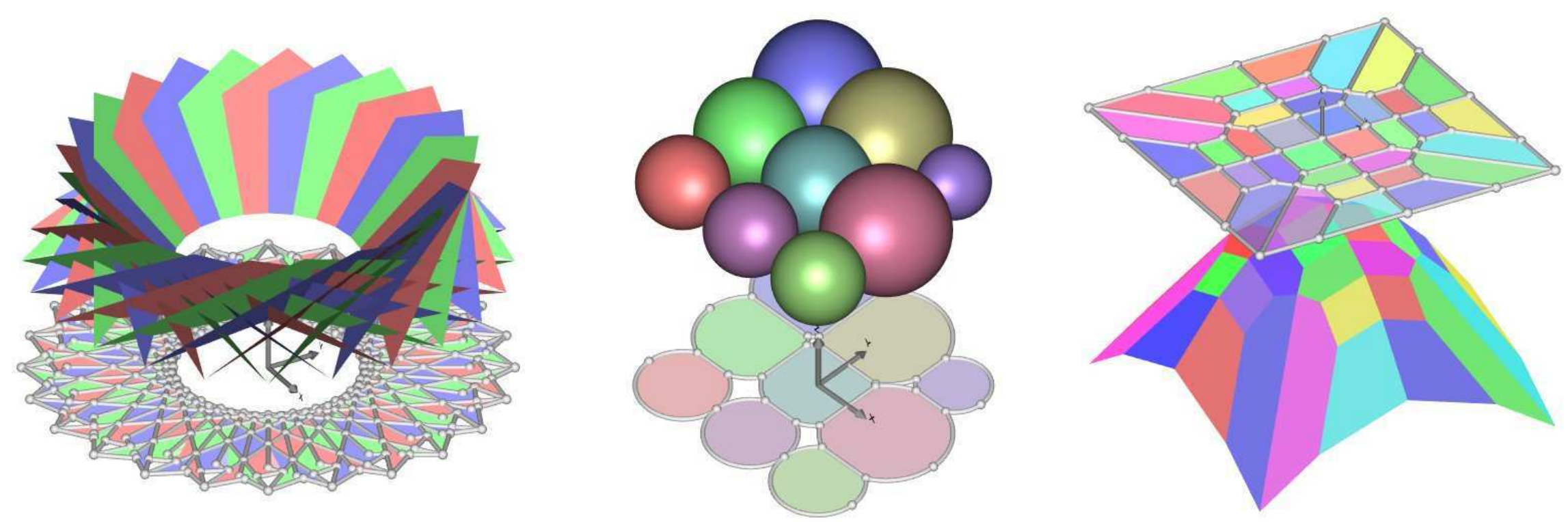


\section{Voronoi Diagrams via Envelopes}

- Computed as lower envelopes of planes

- Represented as planar arrangements of unbounded curves

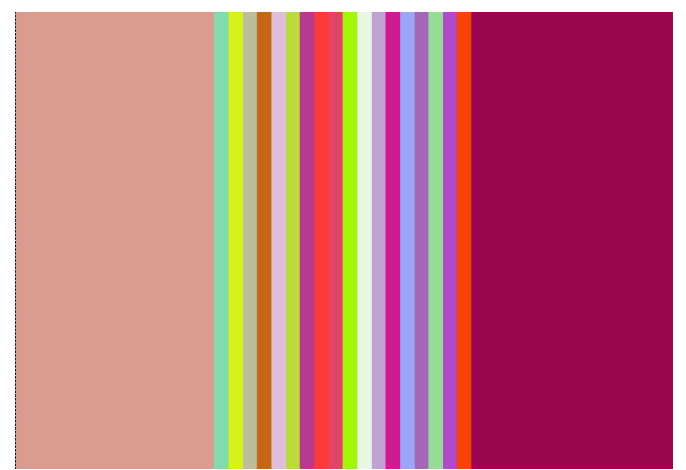

points along a line segment

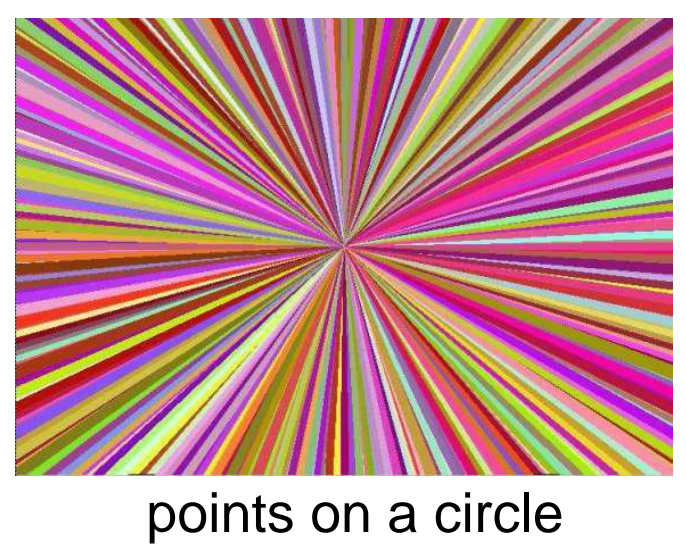

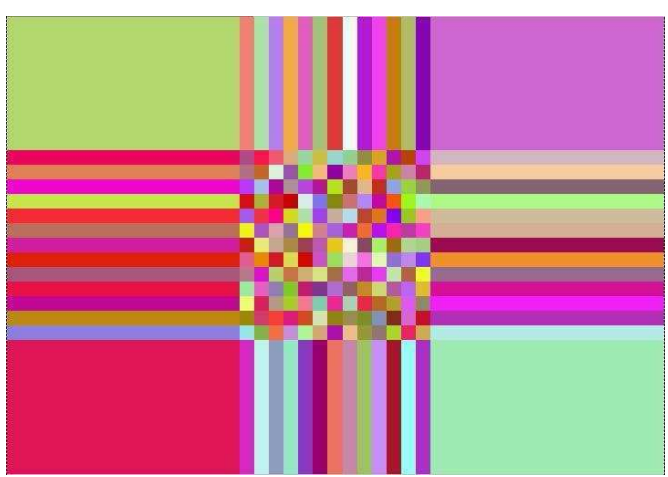

points on a grid inside a square

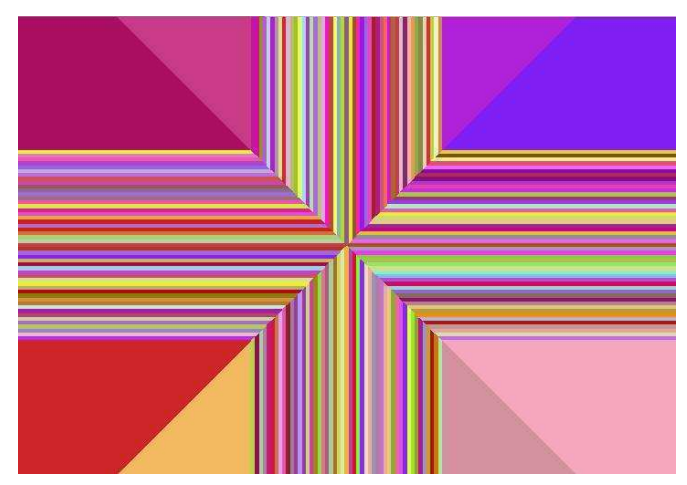

points on a square boundary

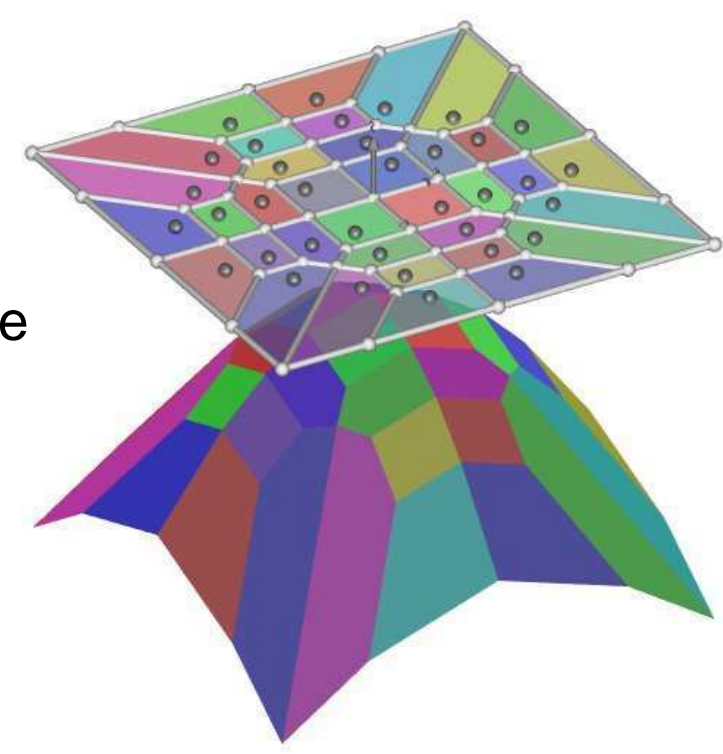




\section{Arrangements on Surfaces in $\mathrm{R}^{3}$}

A parametric surface $S$ of two parameters is a surface defined by parametric equations involving two parameters $u$ and $v$ :

$$
f_{S}(u, v)=(x(u, v), y(u, v), z(u, v))
$$

Thus, $f_{\mathrm{s}}: \mathrm{P} \rightarrow \mathrm{R}^{3}$ and $\mathrm{S}=f_{\mathrm{s}}(\mathrm{P})$, where $\mathrm{P}$ is a continuous and simply connected two-dimensional parameter space

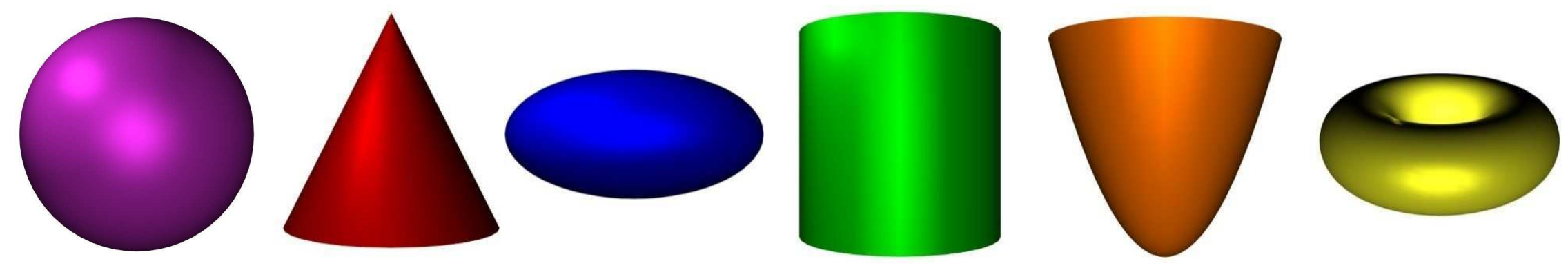

We deal with orientable parametric surfaces 


\section{Minkowski-Sums of Polytopes}

- The Gaussian map of a polytope is the decomposition of $S^{2}$ into maximal connected regions so that the extremal point is the same for all directions within one region

- The overlay of the Gaussian maps of two polytopes $P$ and $Q$ is the Gaussian map of the Minkowski sum of $P$ and $Q$
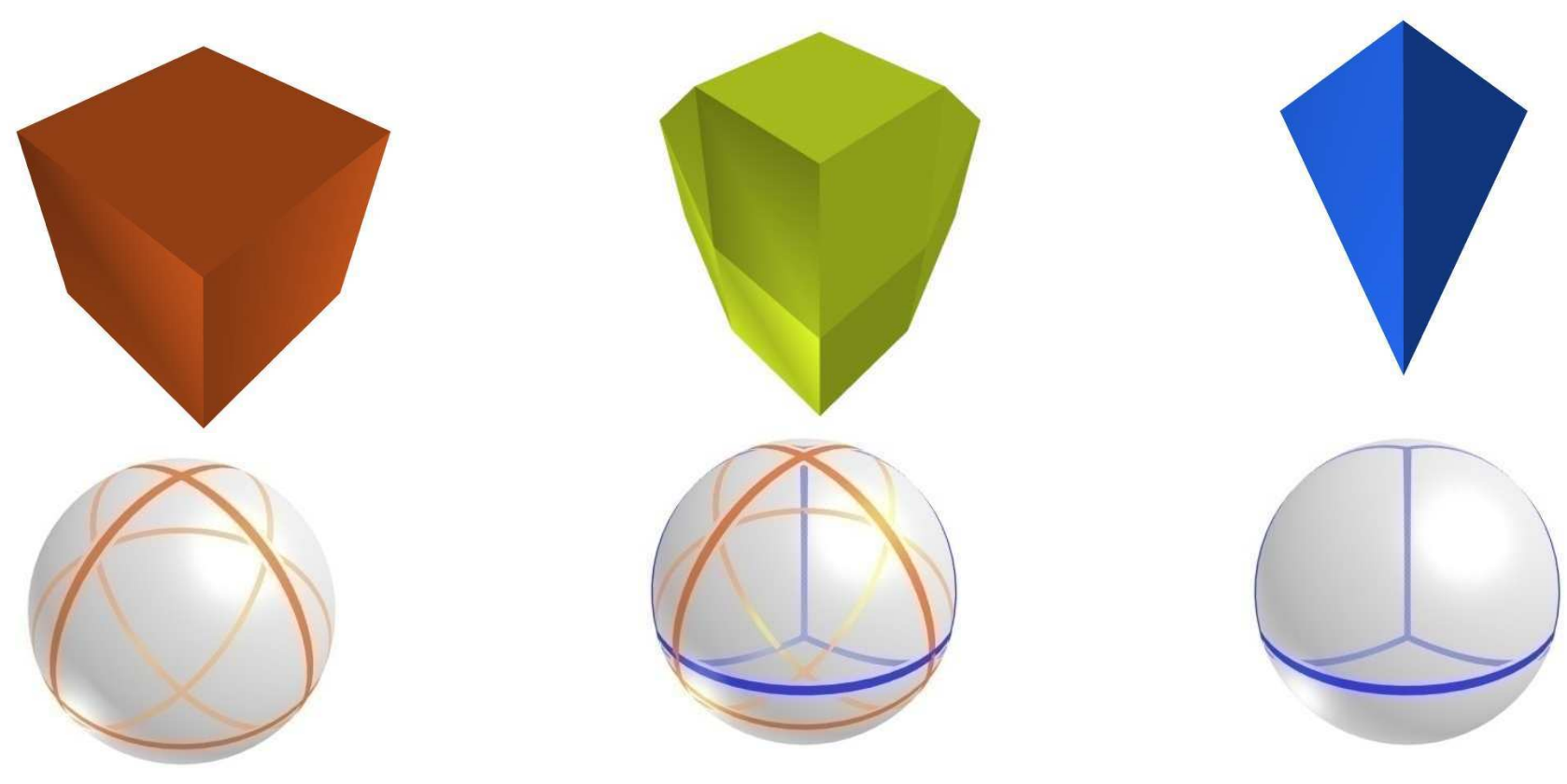


\section{Voronoi Diagrams on the Sphere}

- All algorithms supported by the Arrangement_2 package can also be used on the sphere

- We compute lower envelopes defined over the sphere

- We can compute Voronoi diagrams on the sphere, the edges of which are geodesic arcs

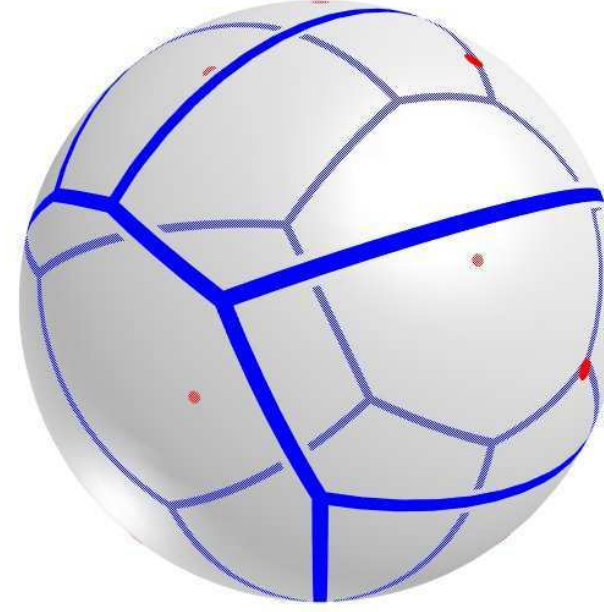

Voronoi diagram on the sphere

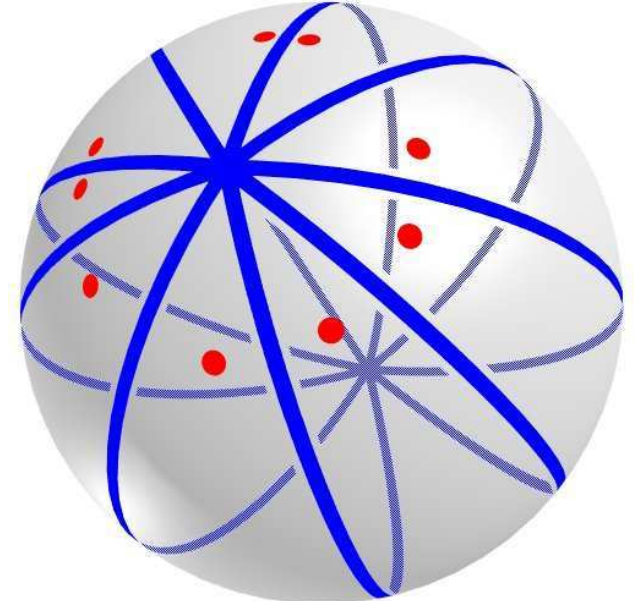

Degenerate Voronoi diagram on the sphere

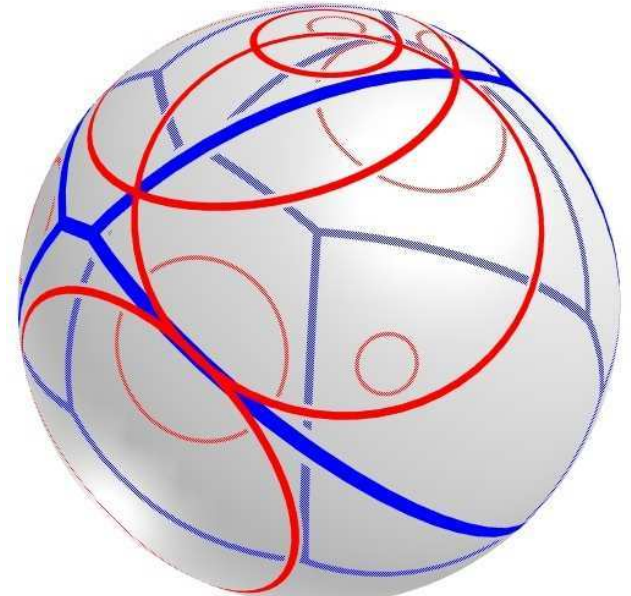

Power (Laguerre Voronoi) diagram on the sphere 


\section{Arrangements on the Sphere}

- The overlay of

- An arrangement on the sphere induced by

- the continents and some of the islands on earth

- 5 cities

- New Orleans

- Los Angeles

- San Antonio

- San Diego

- Boston

- Voronoi diagram of the cities

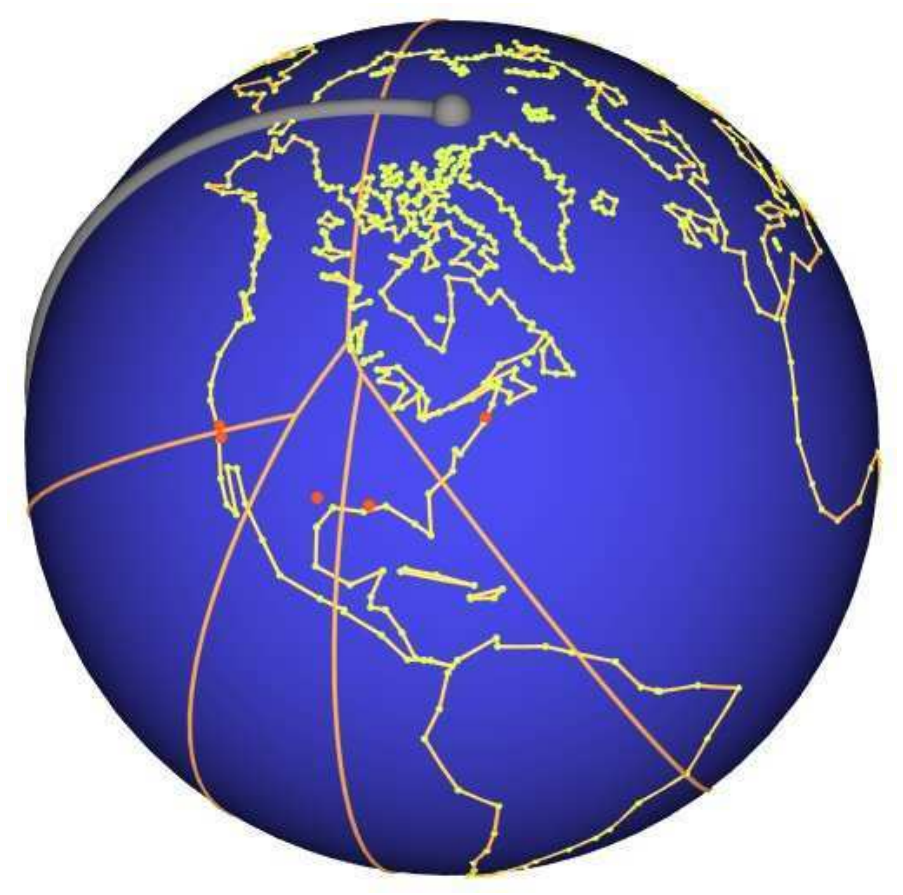

The sphere is oriented such that Cambridge is at the center

- Diagrams, envelopes, etc. are represented as arrangements - Can be passed as input to consecutive operations 


\section{Video}

- Arrangements of Geodesic Arcs on the Sphere

- Was presented at the $24^{\text {th }}$ ACM Symposium on Computational Geometry, College Park, Maryland, July 2008
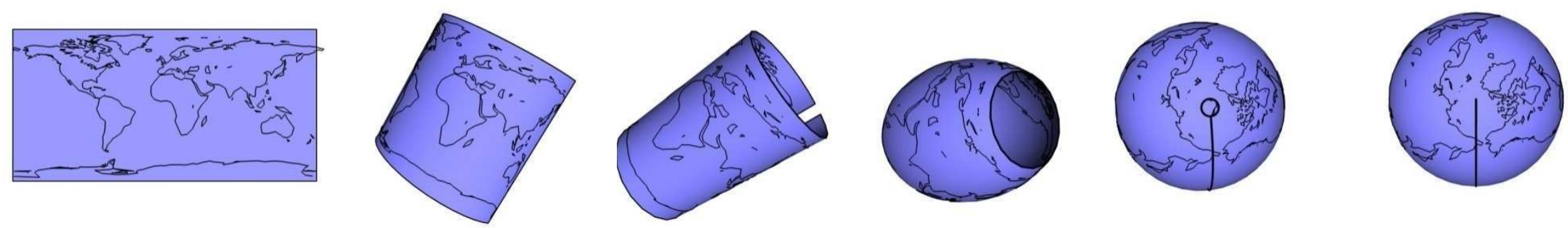


\section{Summary}

- Arrangements are versatile tools

- Arrangements are used as foundation for higher level geometric data structures

- Arrangements are not bound to the plane 


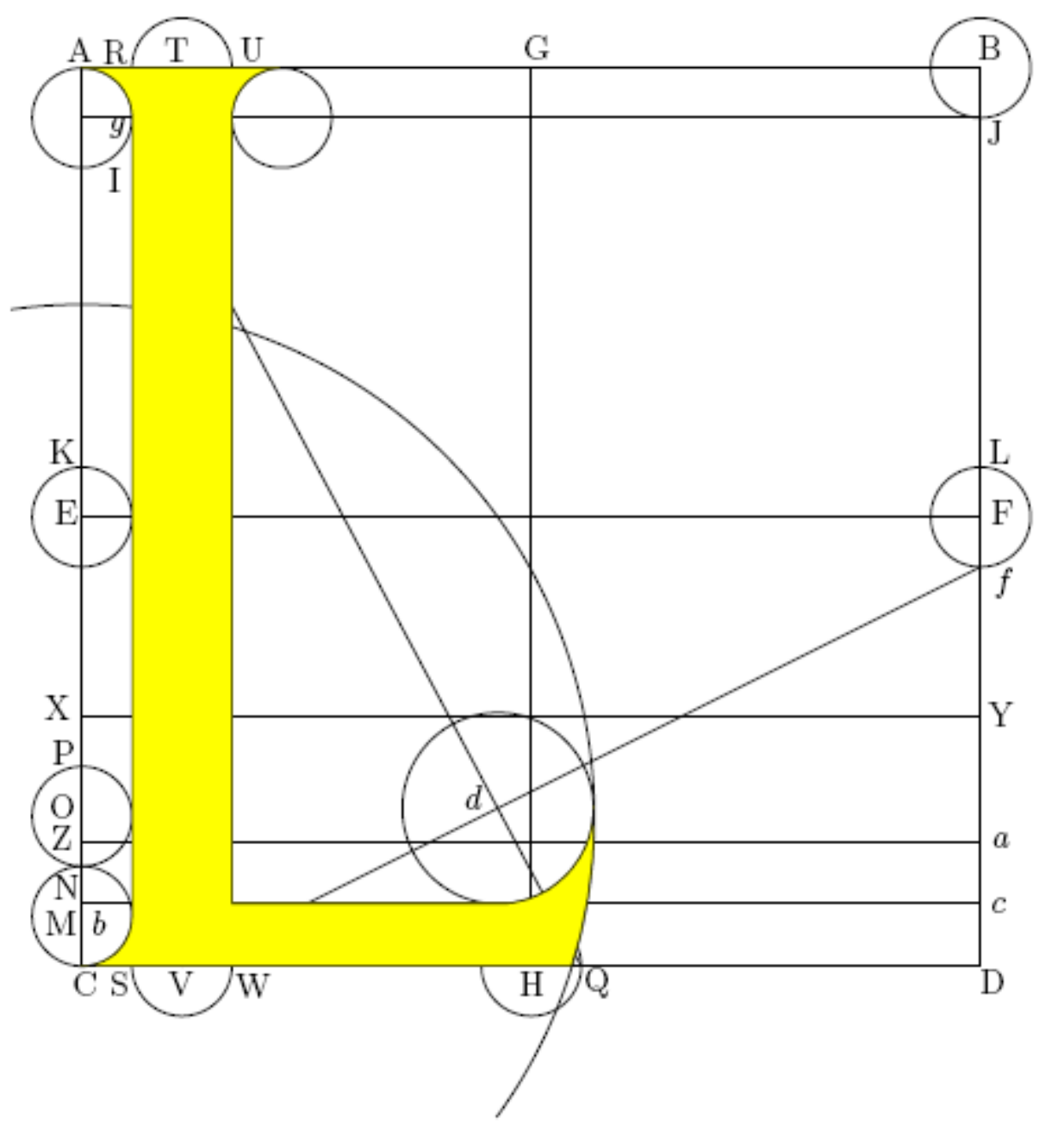

Triangulations and Mesh Generation

Andreas Fabri

GeometryFactory

Pierre Alliez

INRIA 


\section{Outline}

- 2D

- From triangulations to quality meshes

- Related components

- 3D

- Triangulations

- Mesh generation

- Key concepts

- Surfaces

- Volumes

- Next release and work in progress

- Summary 


\section{D}




\section{From Triangulations to Quality Meshes}
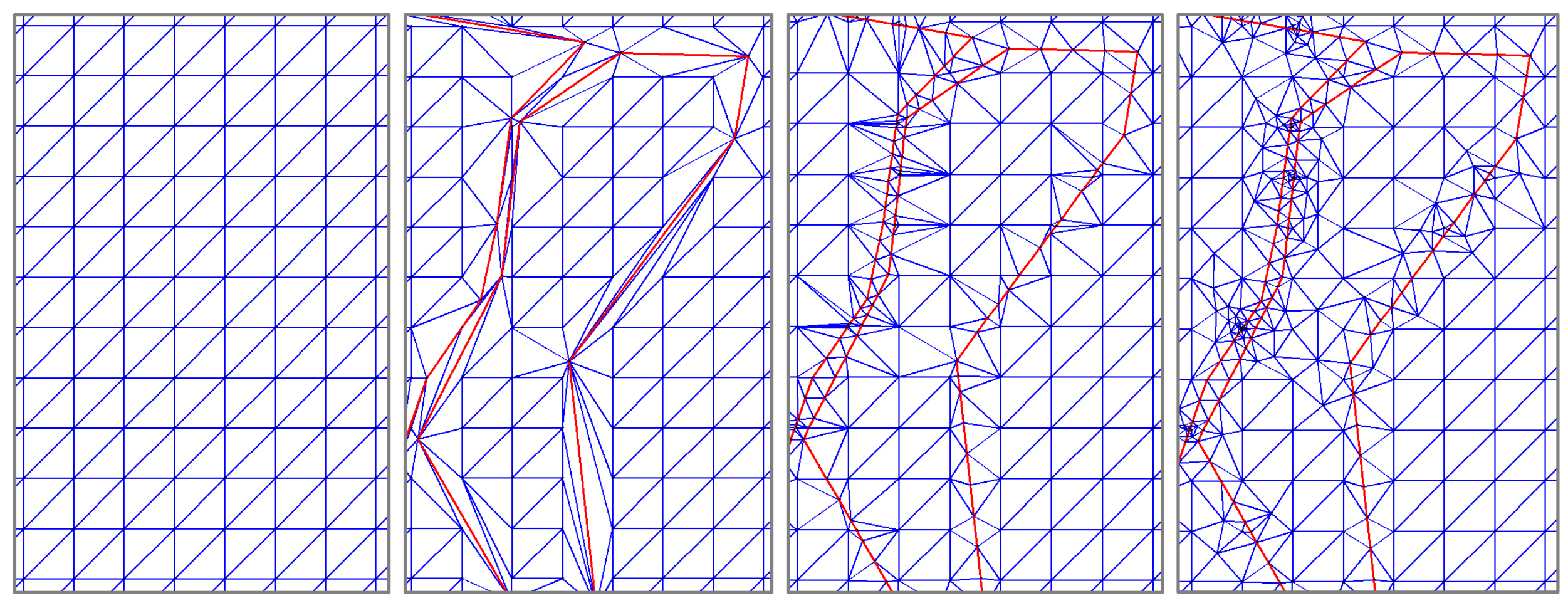


\section{Delaunay Triangulation}

- A triangulation is a Delaunay triangulation, if the circumscribing circle of any facet of the triangulation contains no vertex in its interior

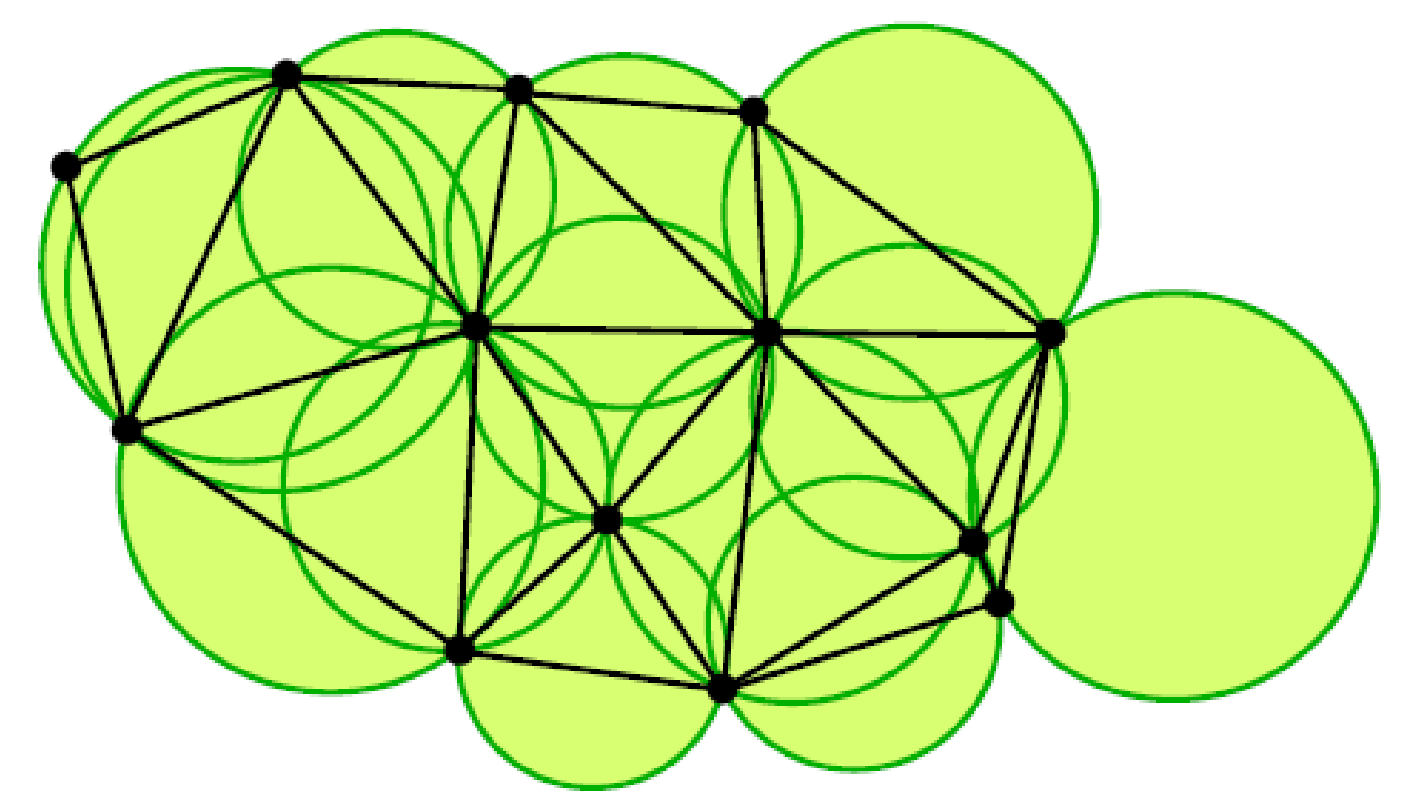




\section{Code Example}

\#include <CGAL/Exact_predicates_inexact_constructions_kernel.h>

\#include <CGAL/Delaunay_triangulation_2.h>

typedef CGAL: :Exact_predicates_inexact_constructions_kernel Kernel;

typedef Kernel::Point_2 Point;

typedef CGAL: :Delaunay_triangulation_2<Kernel> Delaunay;

typedef Delaunay: Vertex_handle Vertex_handle;

int main()

\{

Delaunay dt;

dt.insert( std::istream_iterator<Point>(std: :cin), std: : istream_iterator<Point> () );

Vertex_handle $v=d t$.nearest_vertex (Point $(0.0,0.0))$;

std: : cout $\ll$ "Nearest vertex to origin: " $<$ v->point () $\ll$ std: :endl;

return 0 ;

\} 


\section{Adding Constraints}
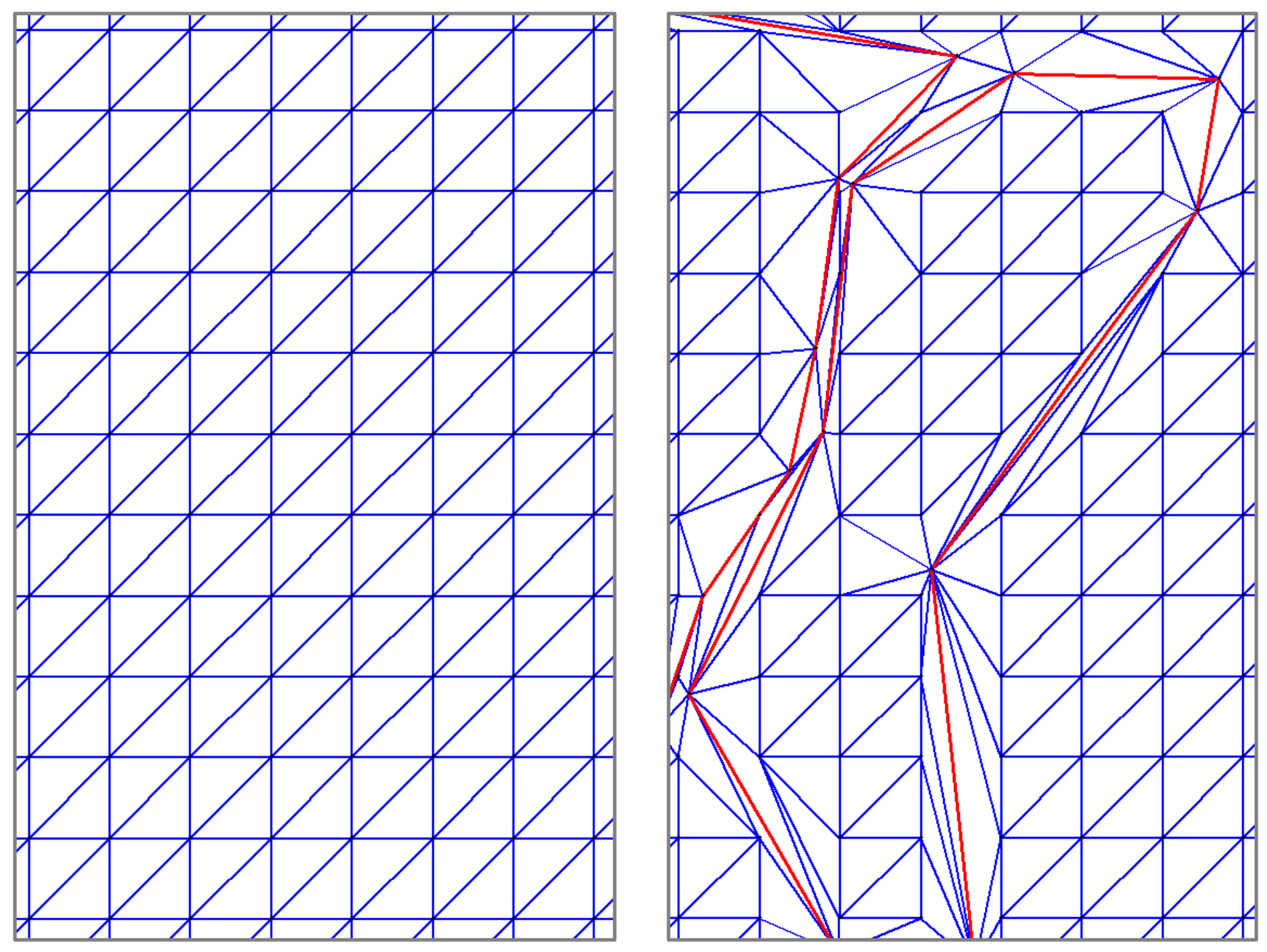

CGAL manual 


\section{Code Example}

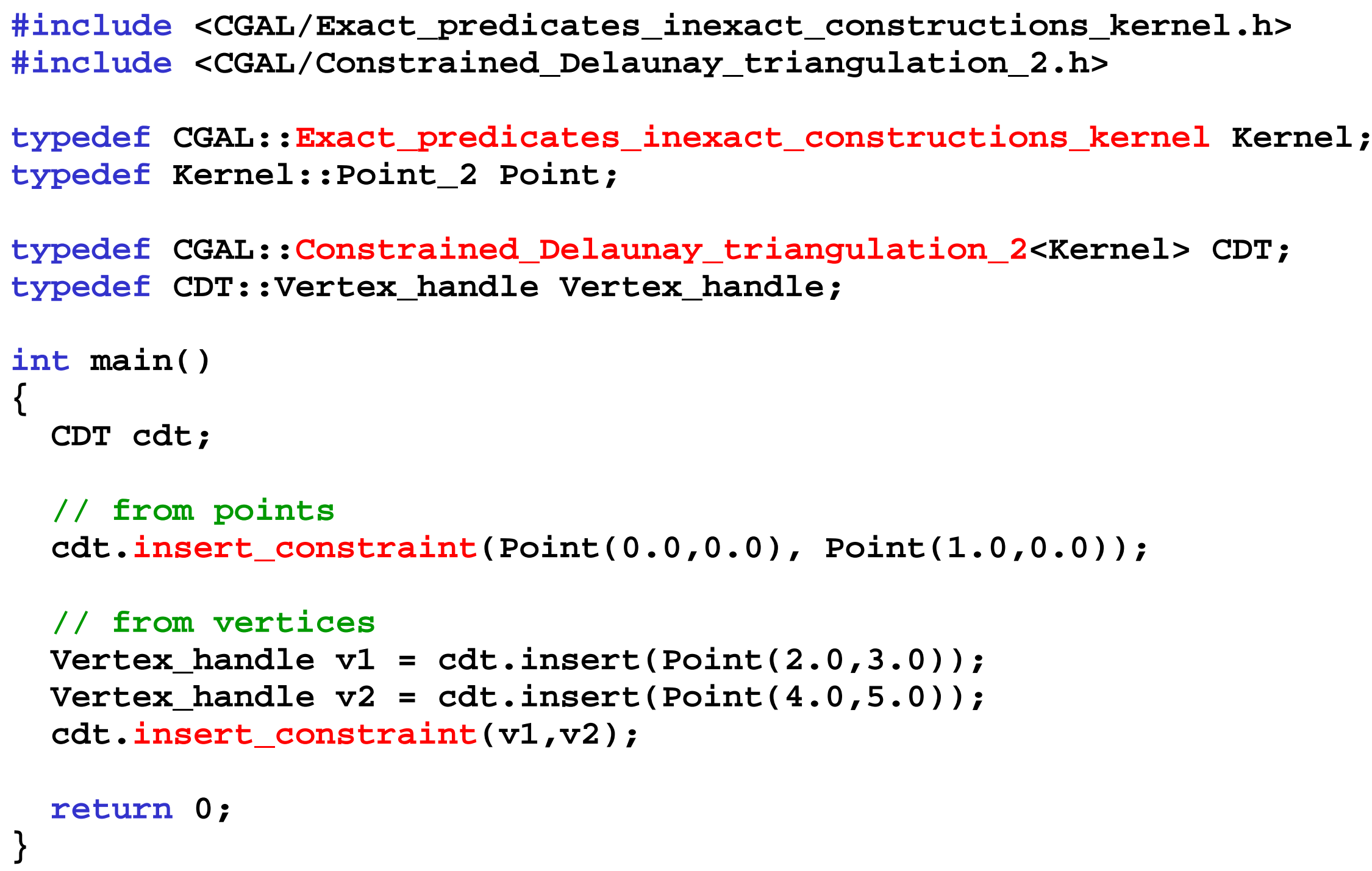




\section{Conforming Delaunay}
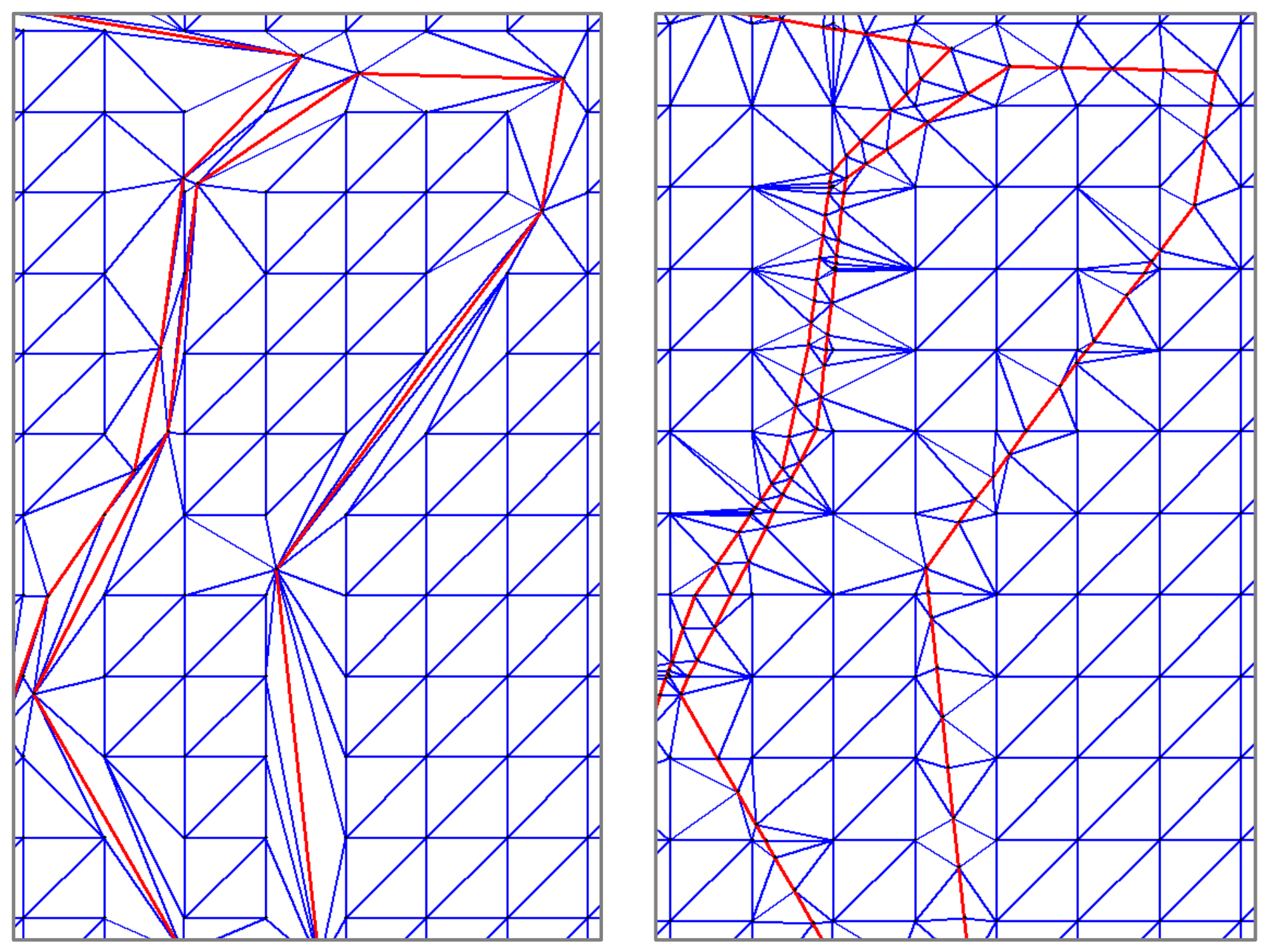

CGAL manual 


\section{Code Example}

\#include <CGAL/Triangulation_conformer_2.h>

// constrained Delaunay triangulation CDT cdt;

... // insert points \& constraints

CGAL : :make_conforming_Delaunay_2 (cdt) ; 


\section{Delaunay Meshing}
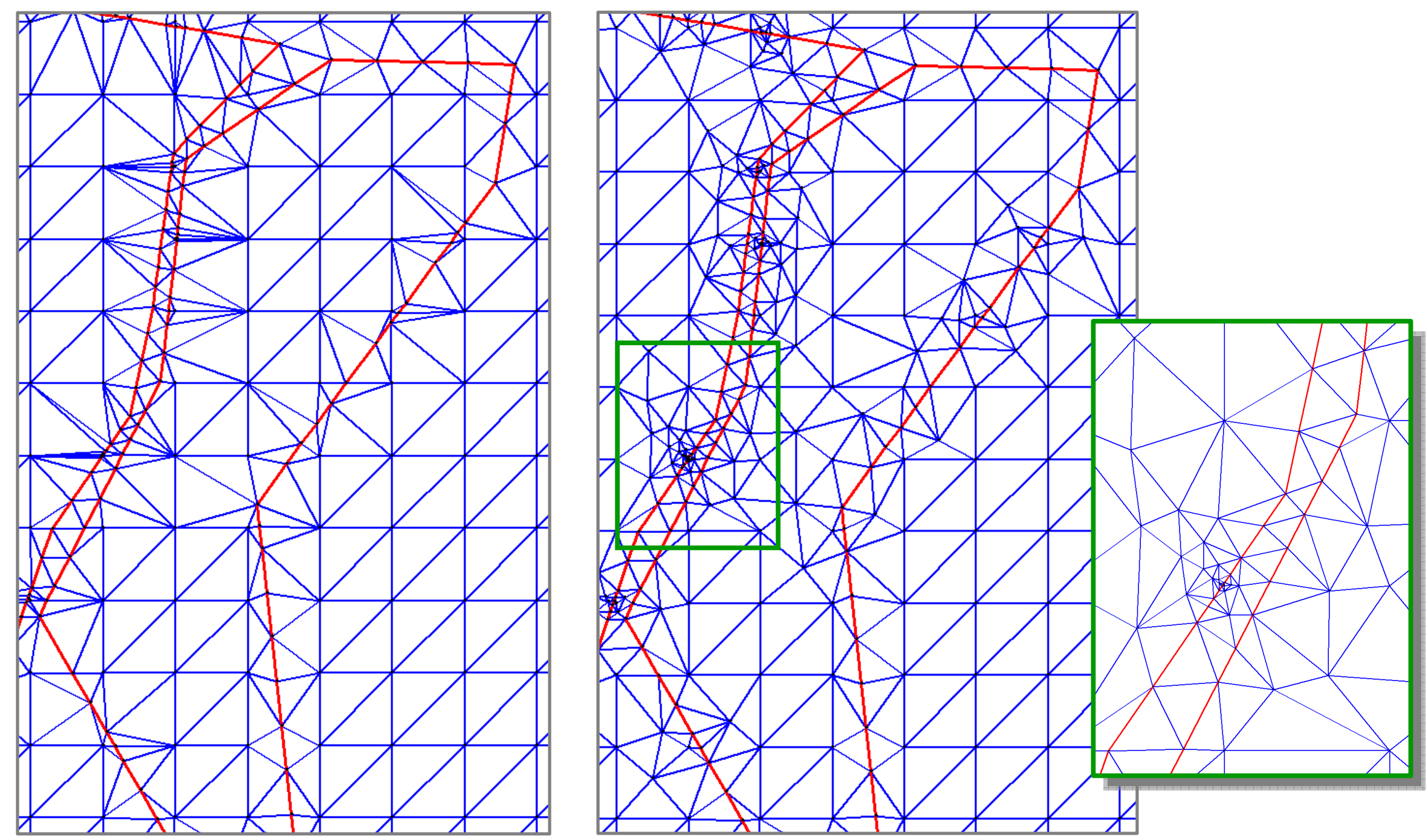


\section{Code Example}

\#include <CGAL/Exact_predicates_inexact_constructions_kernel.h>

\#include <CGAL/Constrained_Delaunay_triangulation_2.h>

\#include <CGAL/Delaunay_mesher_2.h>

typedef CGAL::Exact_predicates_inexact_constructions_kernel Kernel;

typedef CGAL: :Constrained_Delaunay_triangulation_2<Kernel> CDT;

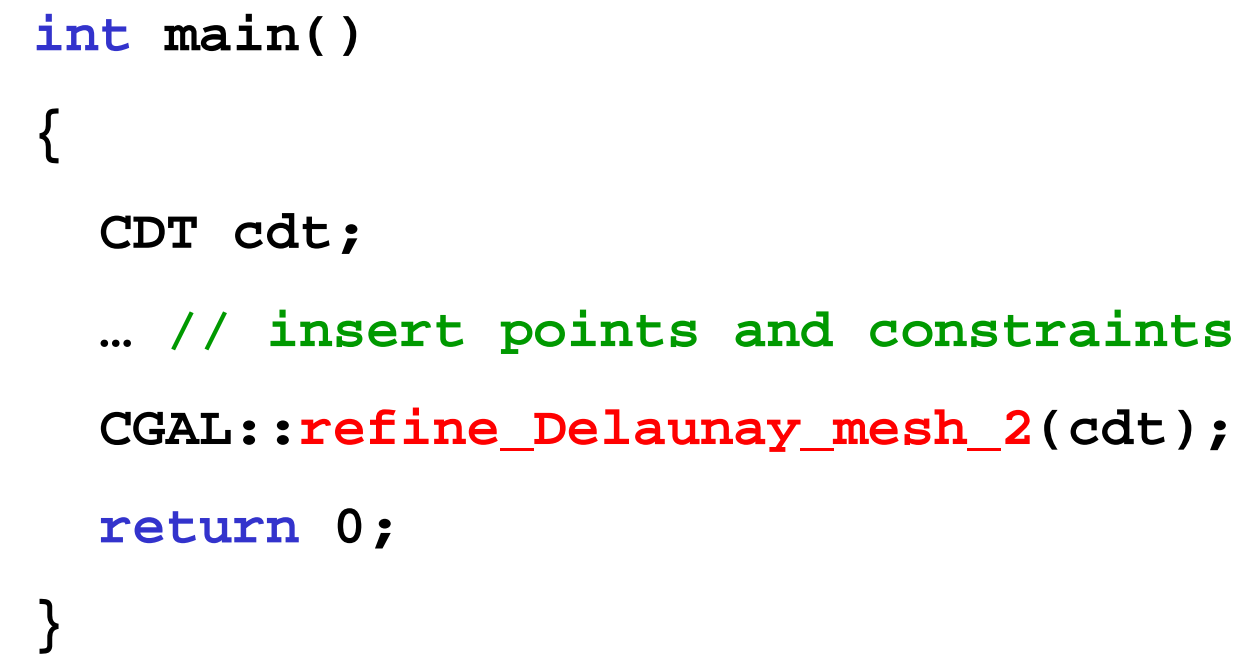




\section{Background}




\section{Delaunay Edge}

An edge is said to be a Delaunay edge, if it is inscribed in an empty circle

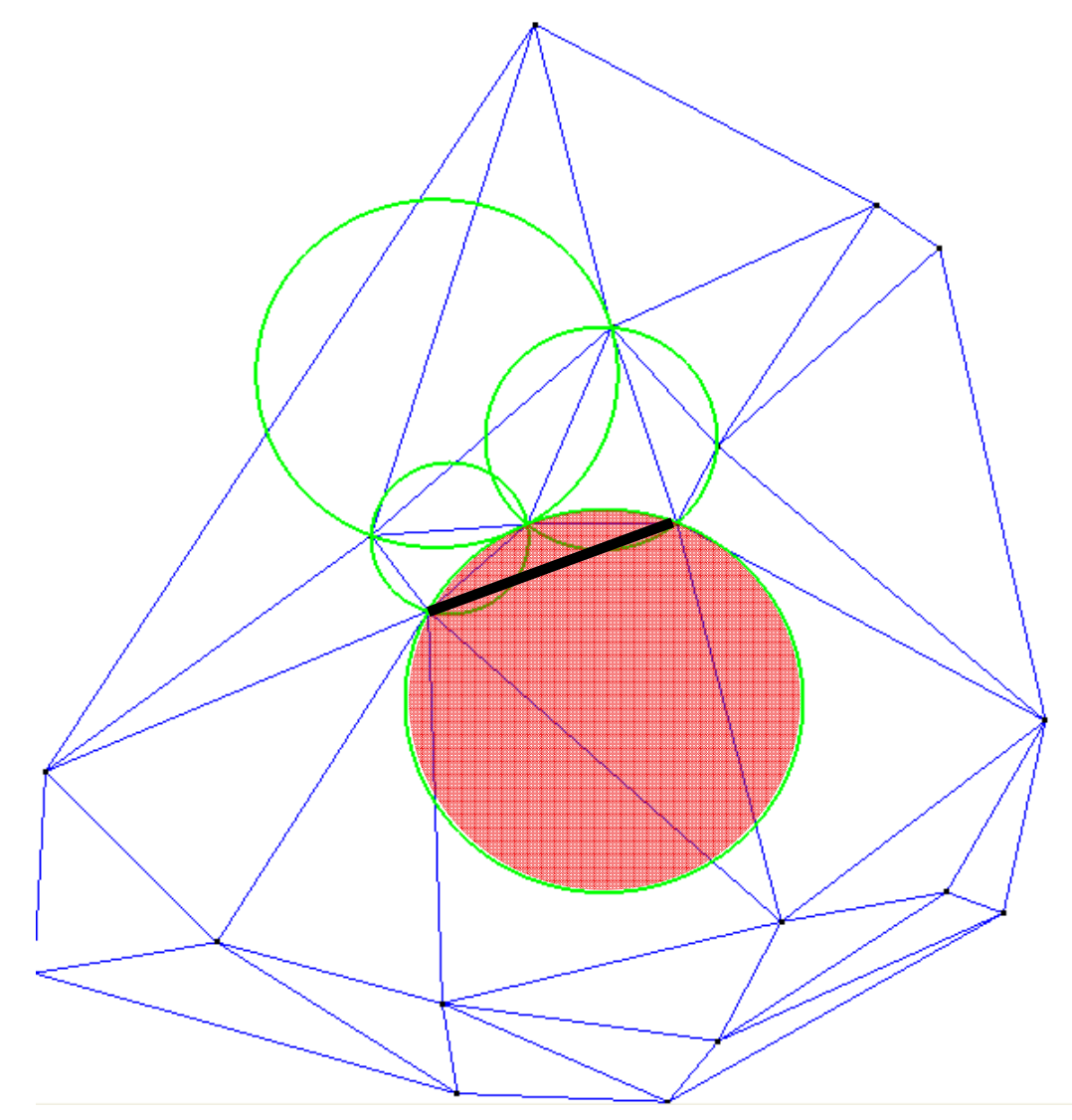




\section{Gabriel Edge}

An edge is said to be a Gabriel edge, if its diametral circle is empty

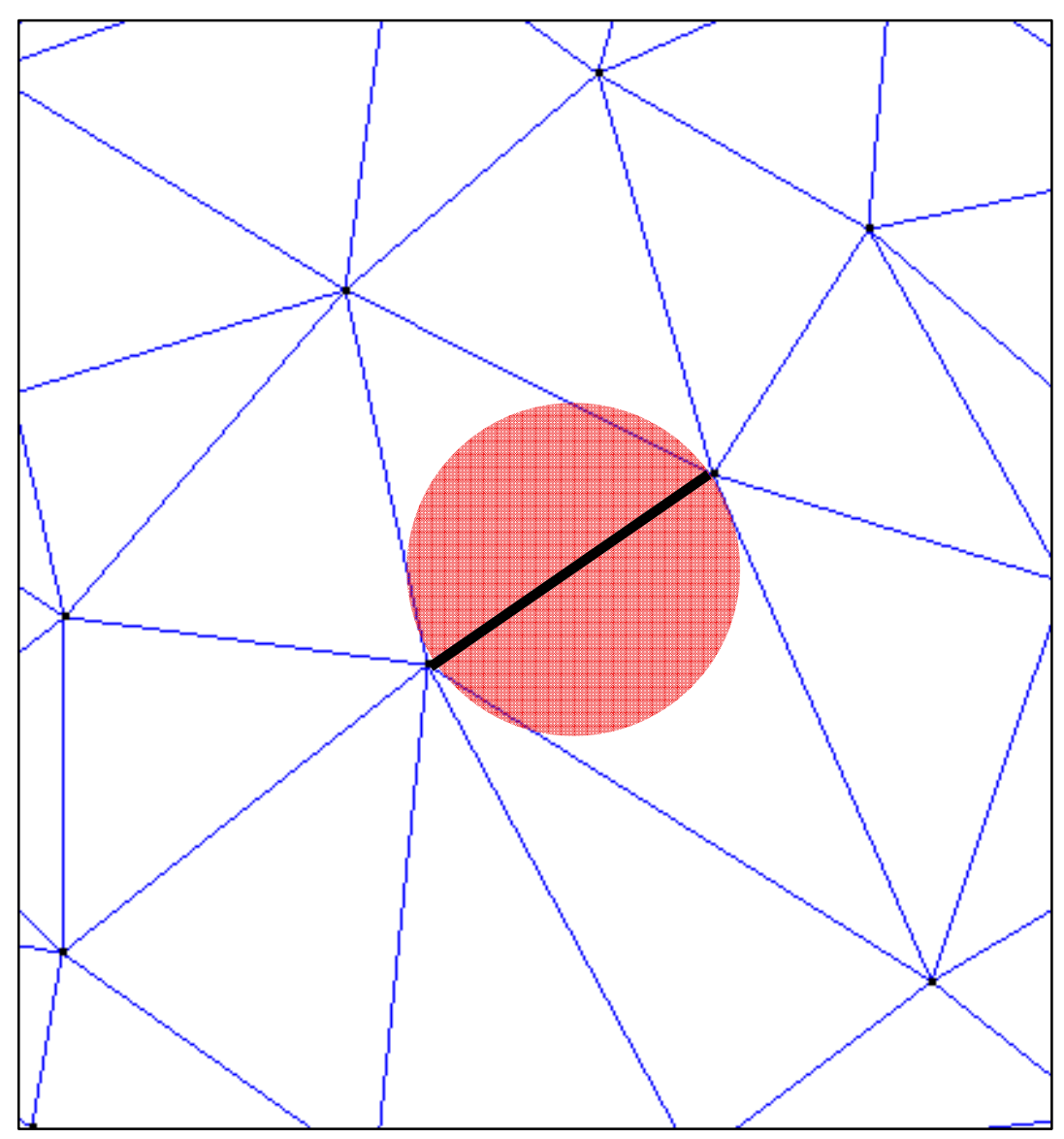




\section{Conforming Delaunay Triangulation}

A constrained Delaunay triangulation is a conforming Delaunay triangulation, if every constrained edge is a Delaunay edge

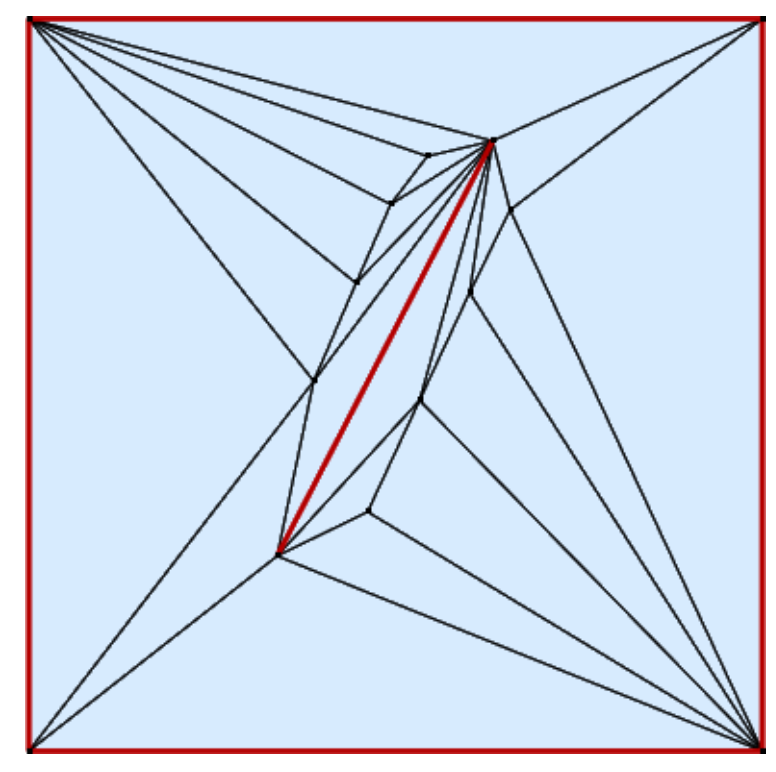

non conforming

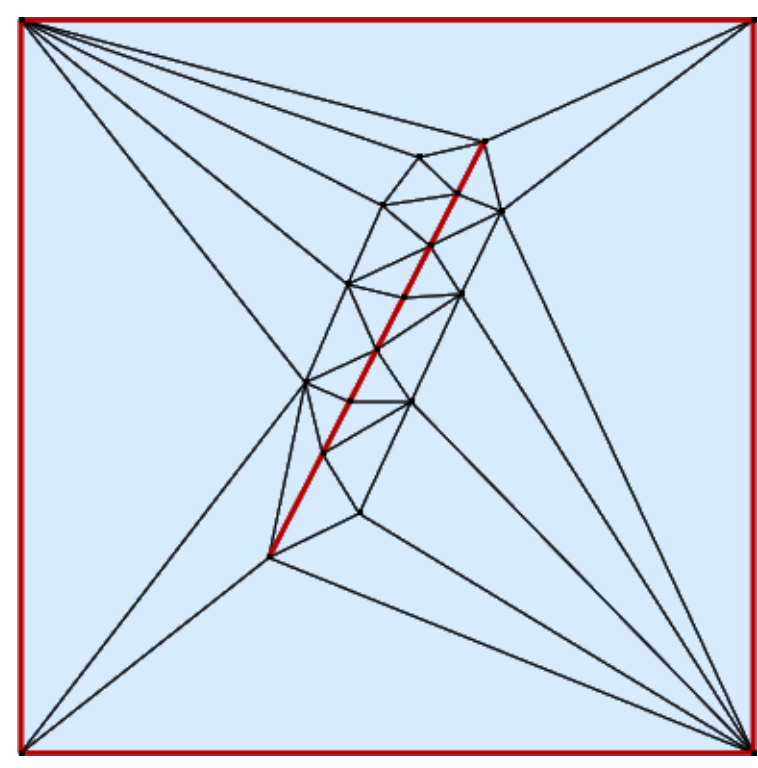

conforming 


\section{Conforming Gabriel Triangulation}

A constrained Delaunay triangulation is a conforming Gabriel triangulation, if every constrained edge is a Gabriel edge

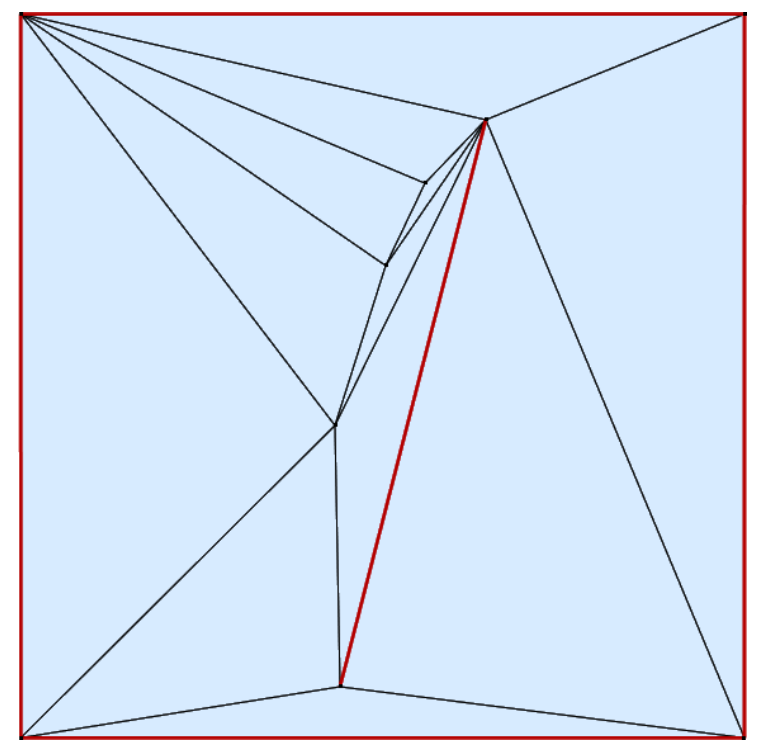

non conforming

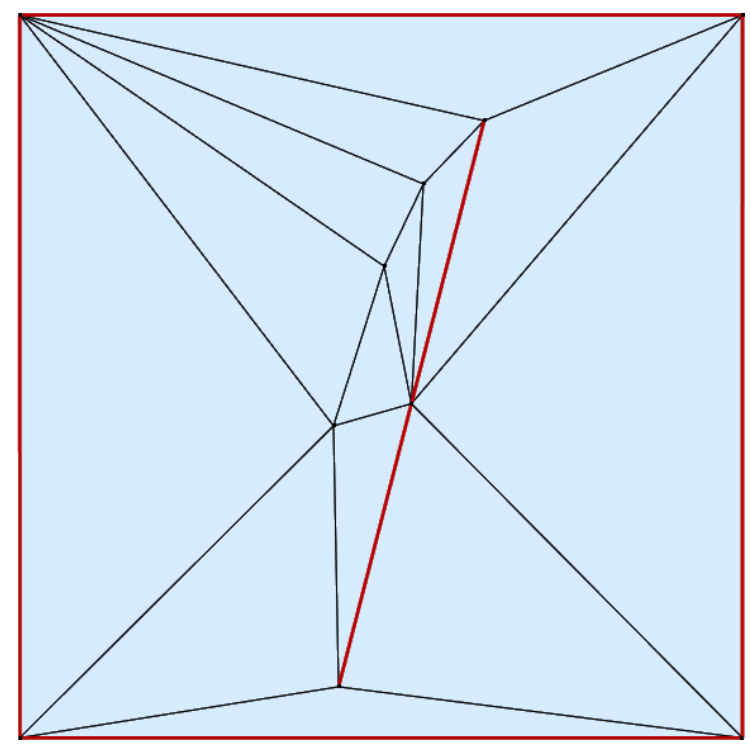

conforming

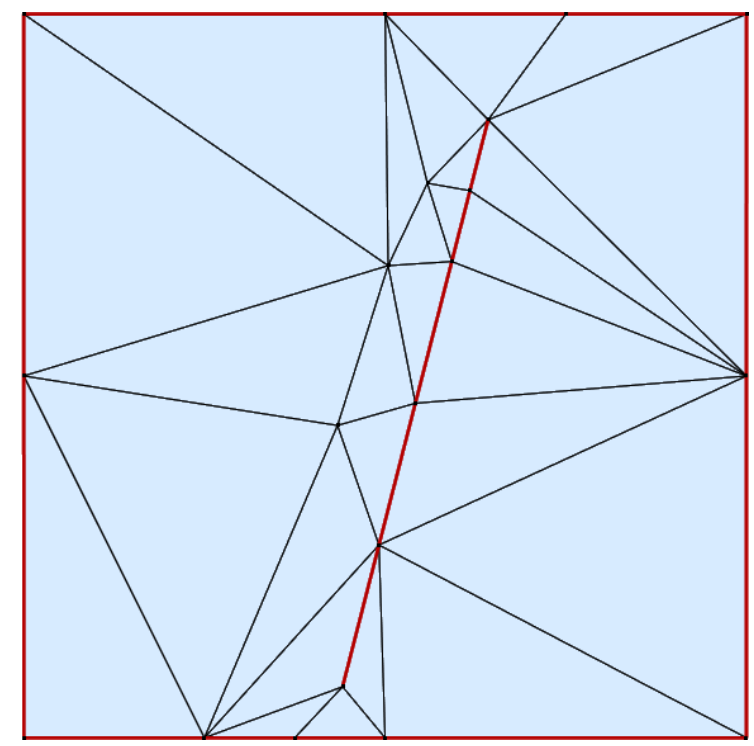

Gabriel 


\section{Steiner Vertices}

Any constrained Delaunay triangulation can be refined into a conforming Delaunay or Gabriel triangulation by adding Steiner vertices.

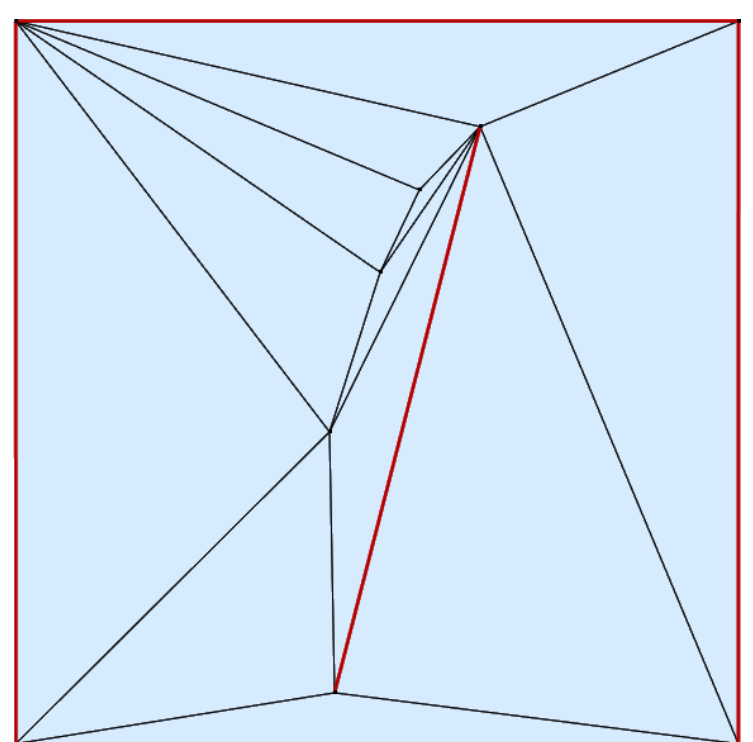

non conforming

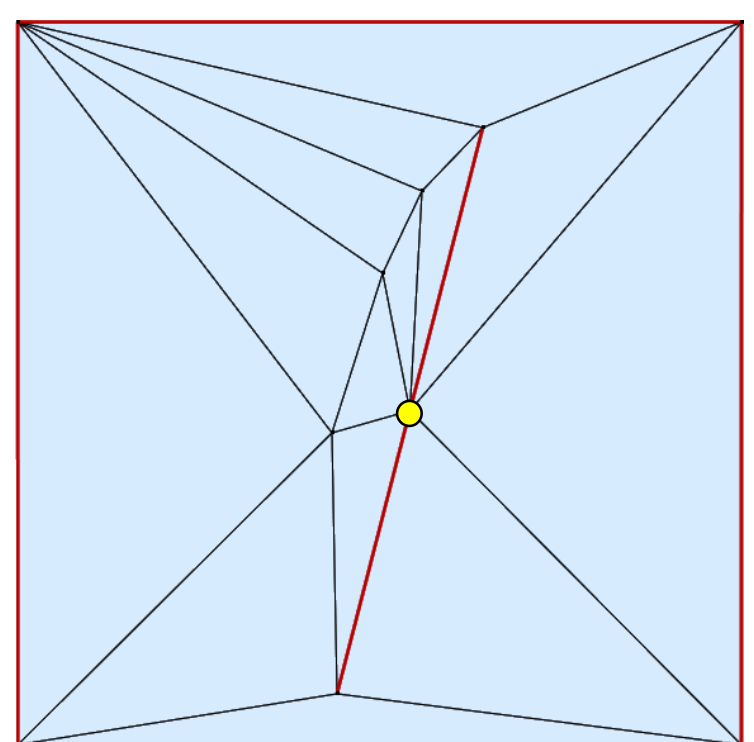

conforming

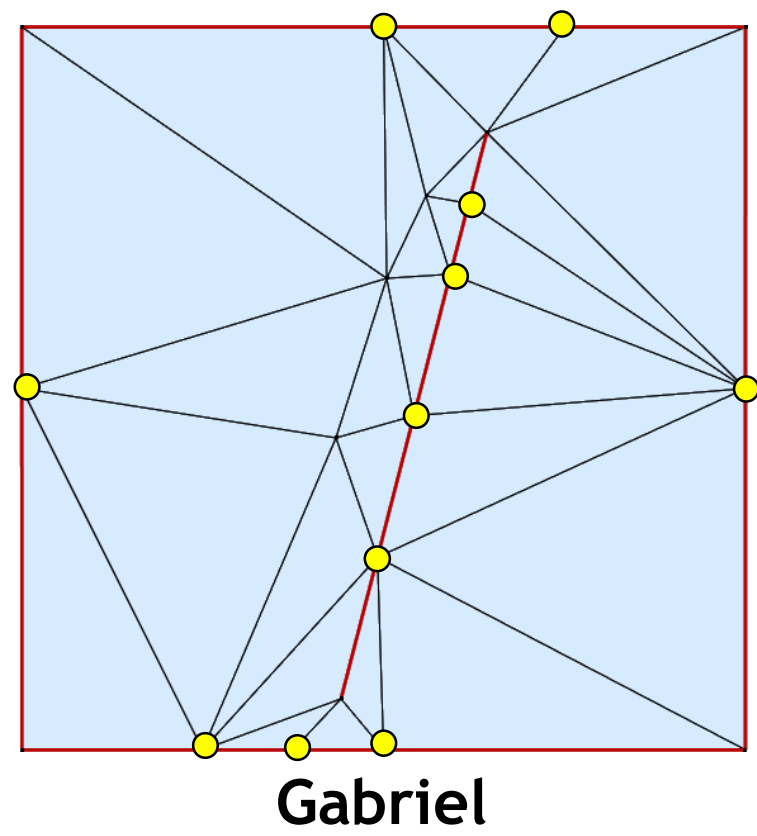




\section{Delaunay Refinement}

Rule \#1: break bad elements by inserting circumcenters (Voronoi vertices)

- "bad" in terms of size or shape (too big or skinny)
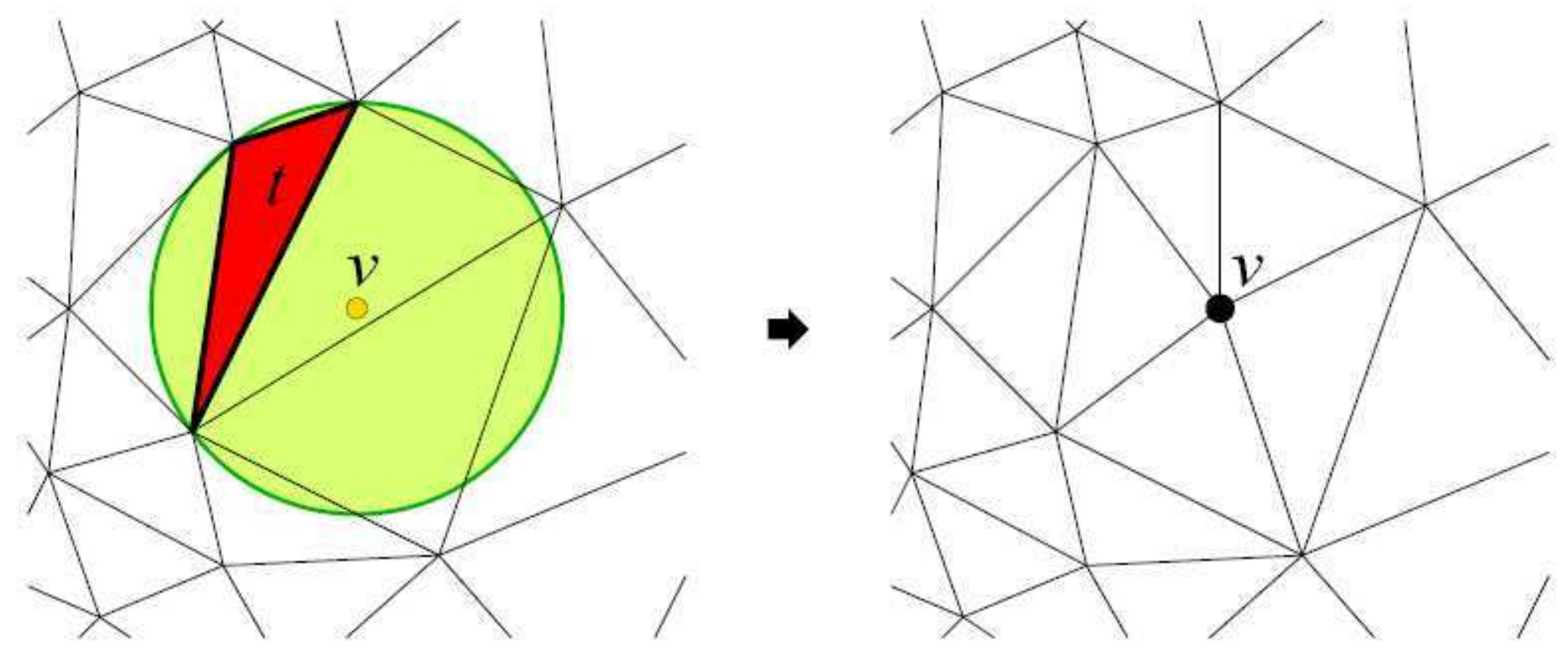

Picture taken from [Shewchuk] 


\section{Delaunay Refinement}

Rule \#2: Midpoint vertex insertion

A constrained segment is said to be encroached, if there is a vertex inside its diametral circle
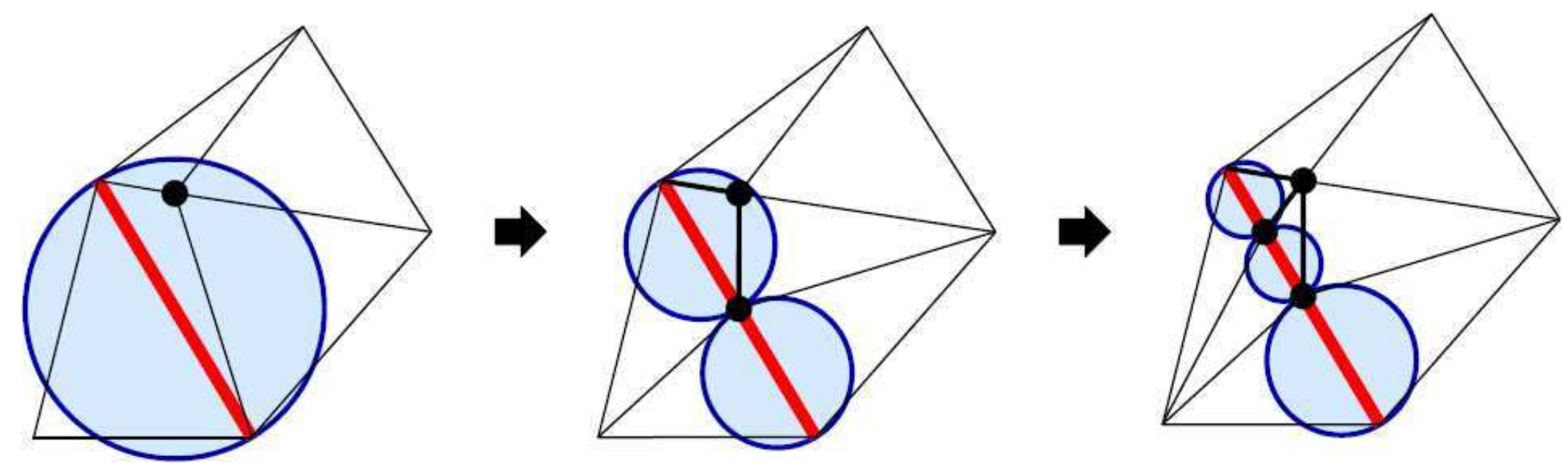


\section{Delaunay Refinement}

Encroached subsegments have priority over skinny triangles

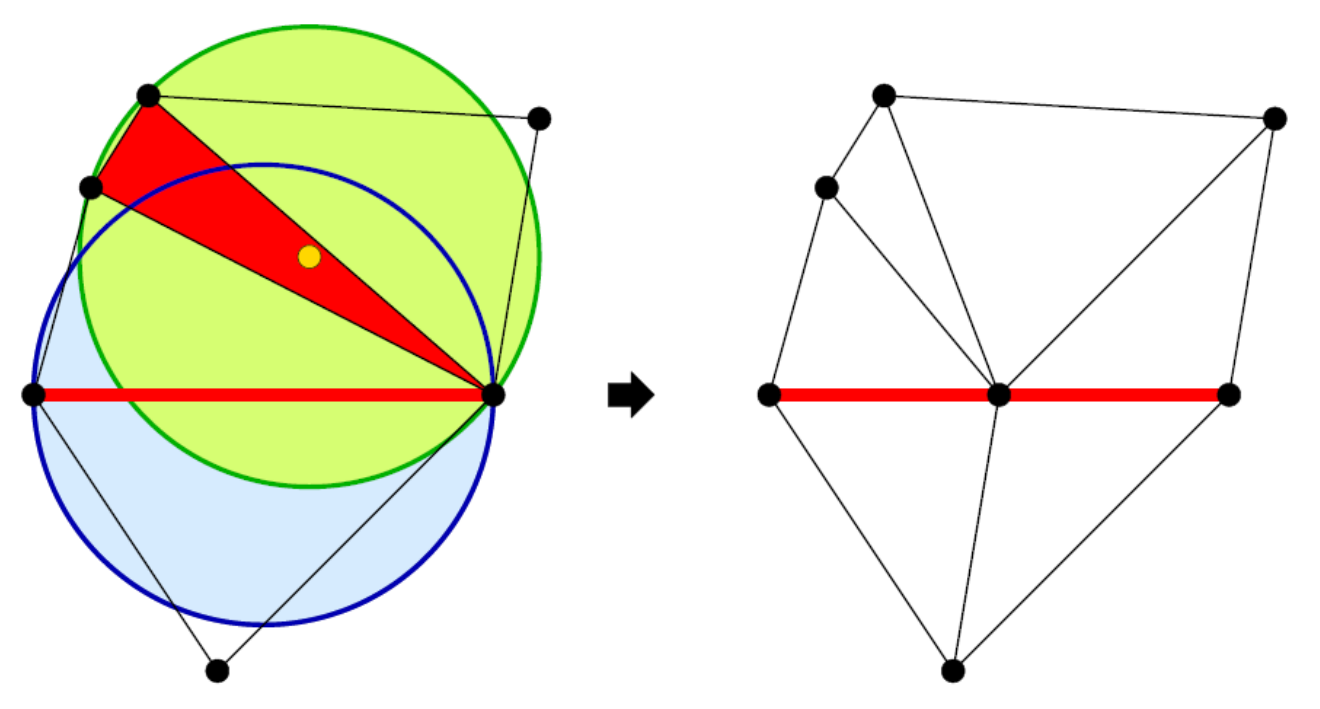


API 
Rich API

- Traversal

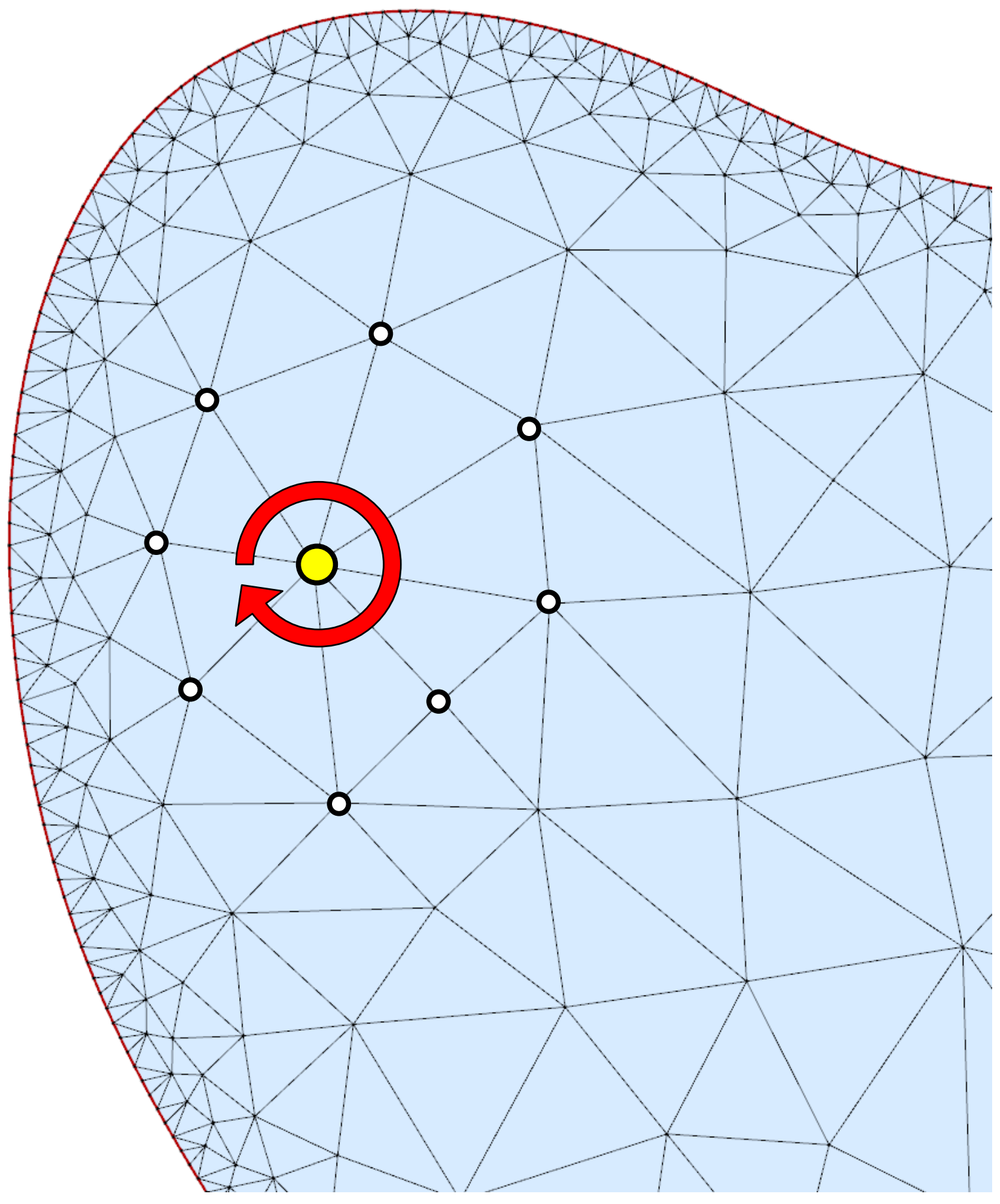


Rich API

- Traversal

- Localization

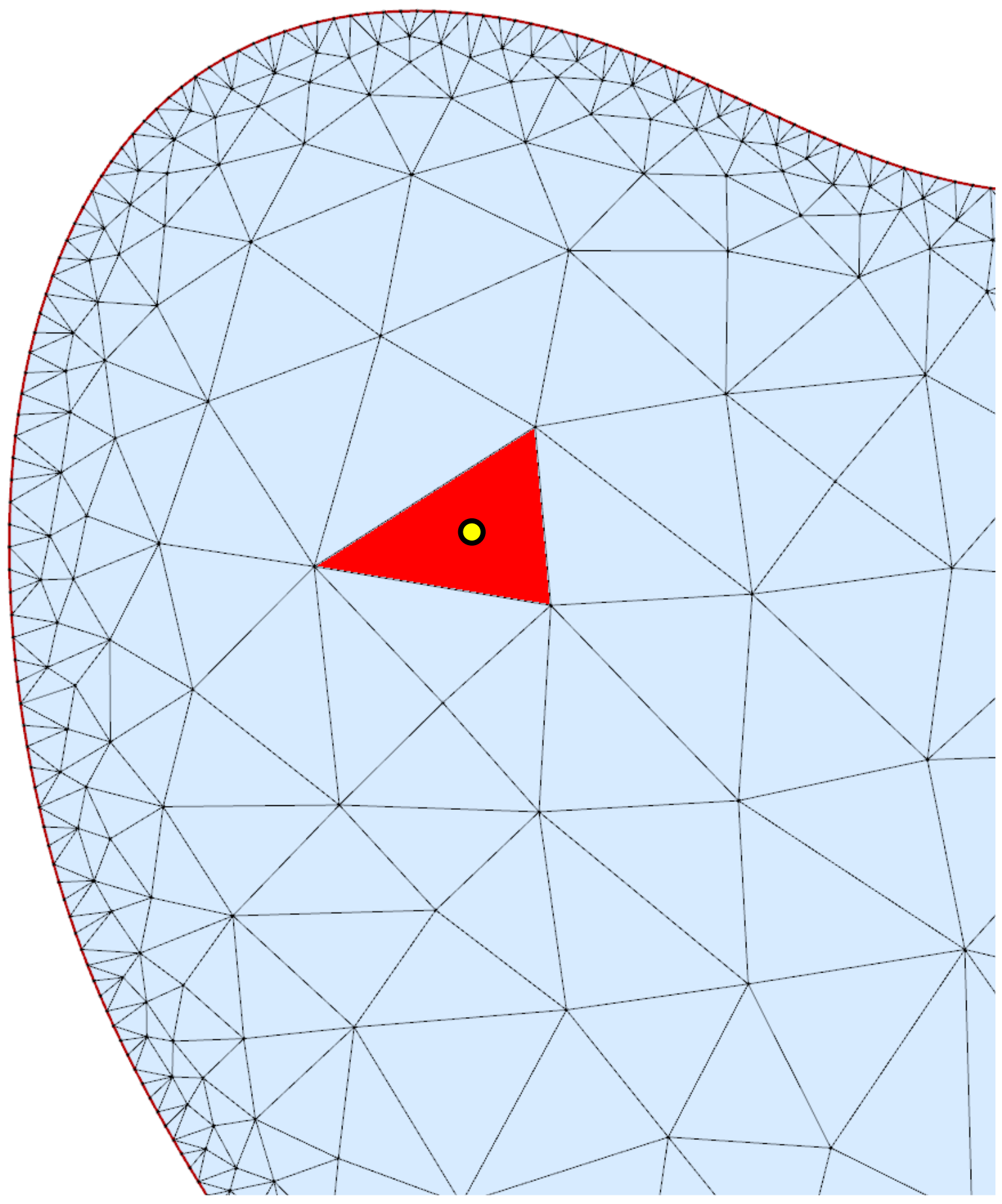




\section{Rich API}

- Traversal

- Localization

- Dynamic: insertion \& removal
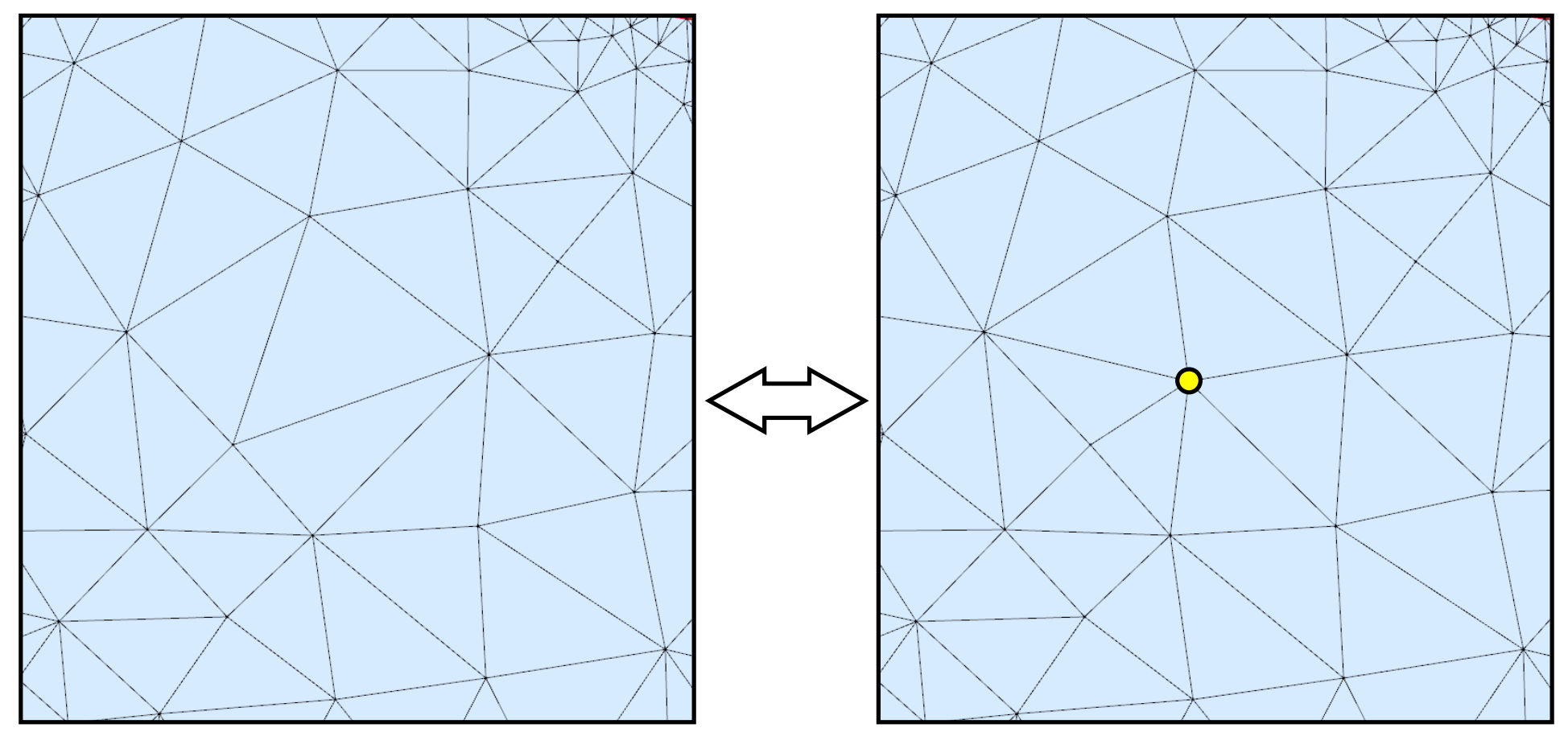


\section{Rich API}

- Traversal

- Localization

- Dynamic: insertion \& removal

- Parameters for mesh generation 


\section{Parameters for Mesh Generation}

- Shape

- Lower bound on triangle angles

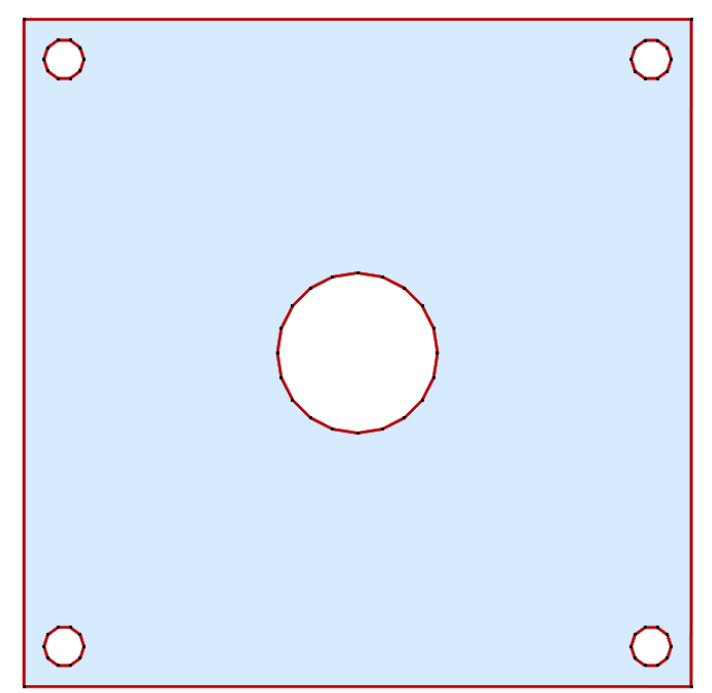

Input PLSG

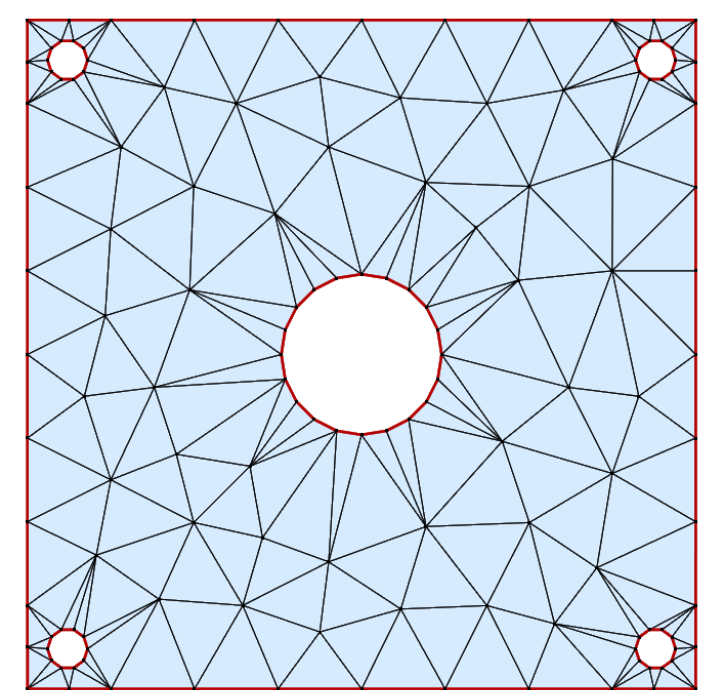

5 deg
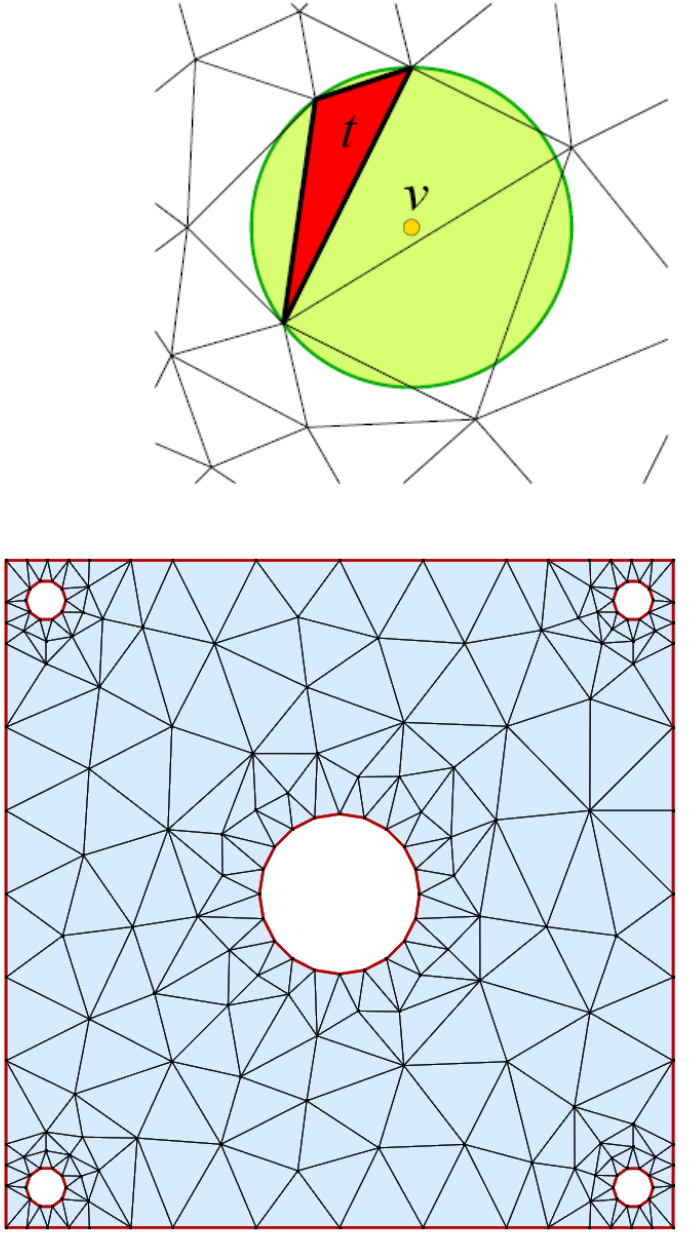

$20.7 \mathrm{deg}$

Online manual 


\section{Parameters for Mesh Generation}

- Shape

- Lower bound on triangle angles

- Size

- No constraint

- Uniform sizing

- Sizing function

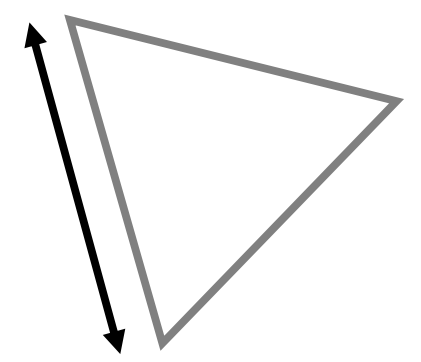




\section{Sizing Parameter}

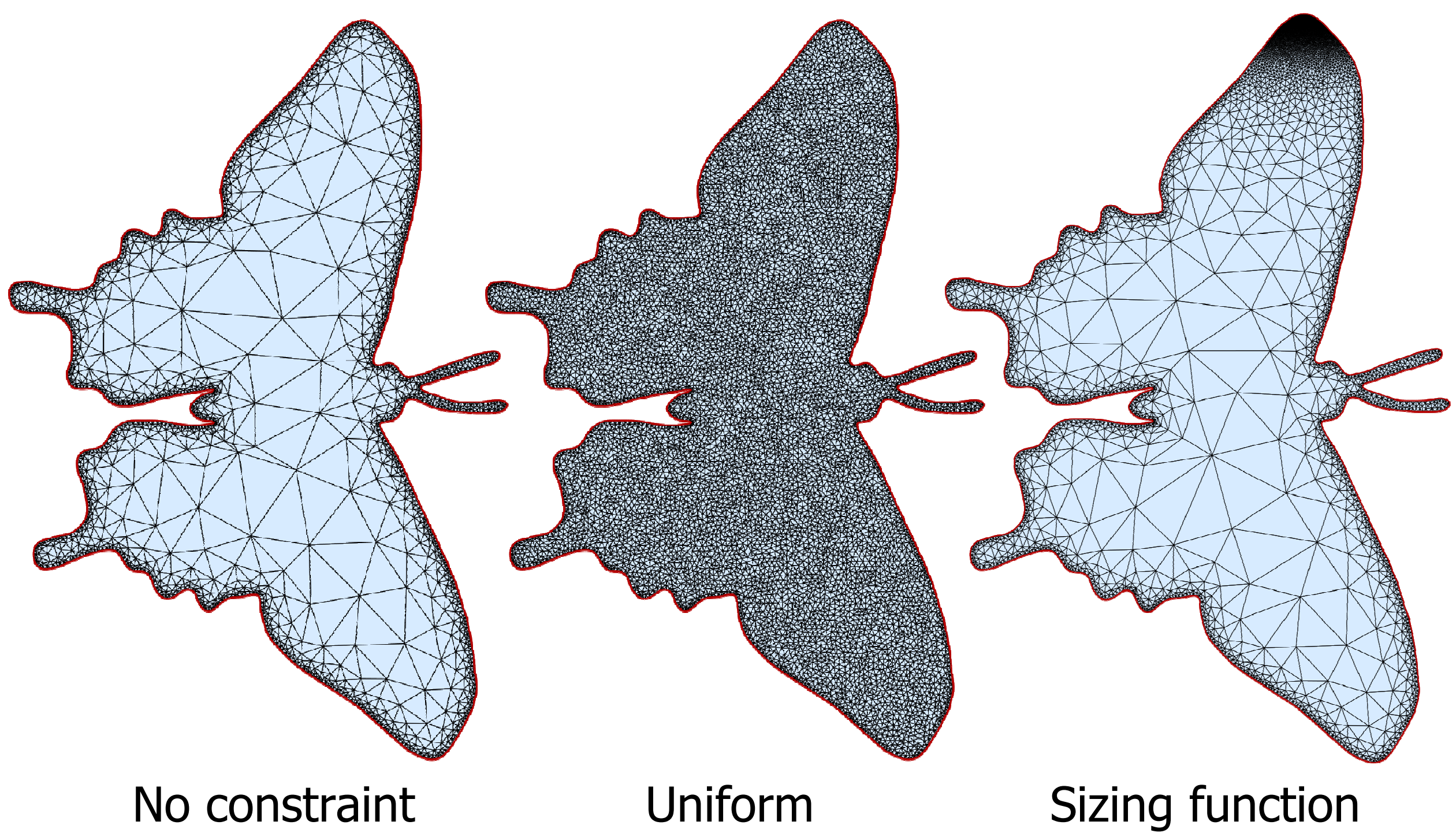




\section{Example Code}

\#include <CGAL/Exact_predicates_inexact_constructions_kernel.h>

\#include <CGAL/Constrained_Delaunay_triangulation_2.h>

\#include <CGAL/Delaunay_mesher_2.h>

\#include <CGAL/Delaunay_mesh_size_criteria_2.h>

typedef CGAL::Exact_predicates_inexact_constructions_kernel Kernel;

typedef CGAL::Constrained_Delaunay_triangulation_2<Kernel> CDT;

typedef CGAL: :Delaunay_mesh_size_criteria_2<CDT> Criteria;

typedef CGAL: :Delaunay_mesher_2<CDT, Criteria> Meshing_engine;

int main ()

\{

CDT cdt;

Meshing_engine engine(cdt);

engine.refine_mesh ();

engine.set_criteria(Criteria(0.125, 0.5));// min 20.6 deg

$/ / 0.5$ for sizing

engine.refine_mesh(); // refine once more, etc.

return 0 ; 


\section{Parameters for Mesh Generation}

- Shape

- Lower bound on triangle angles

- Size

- No constraint

- Uniform sizing

- Sizing function

- Seeds

- Exclude/include components

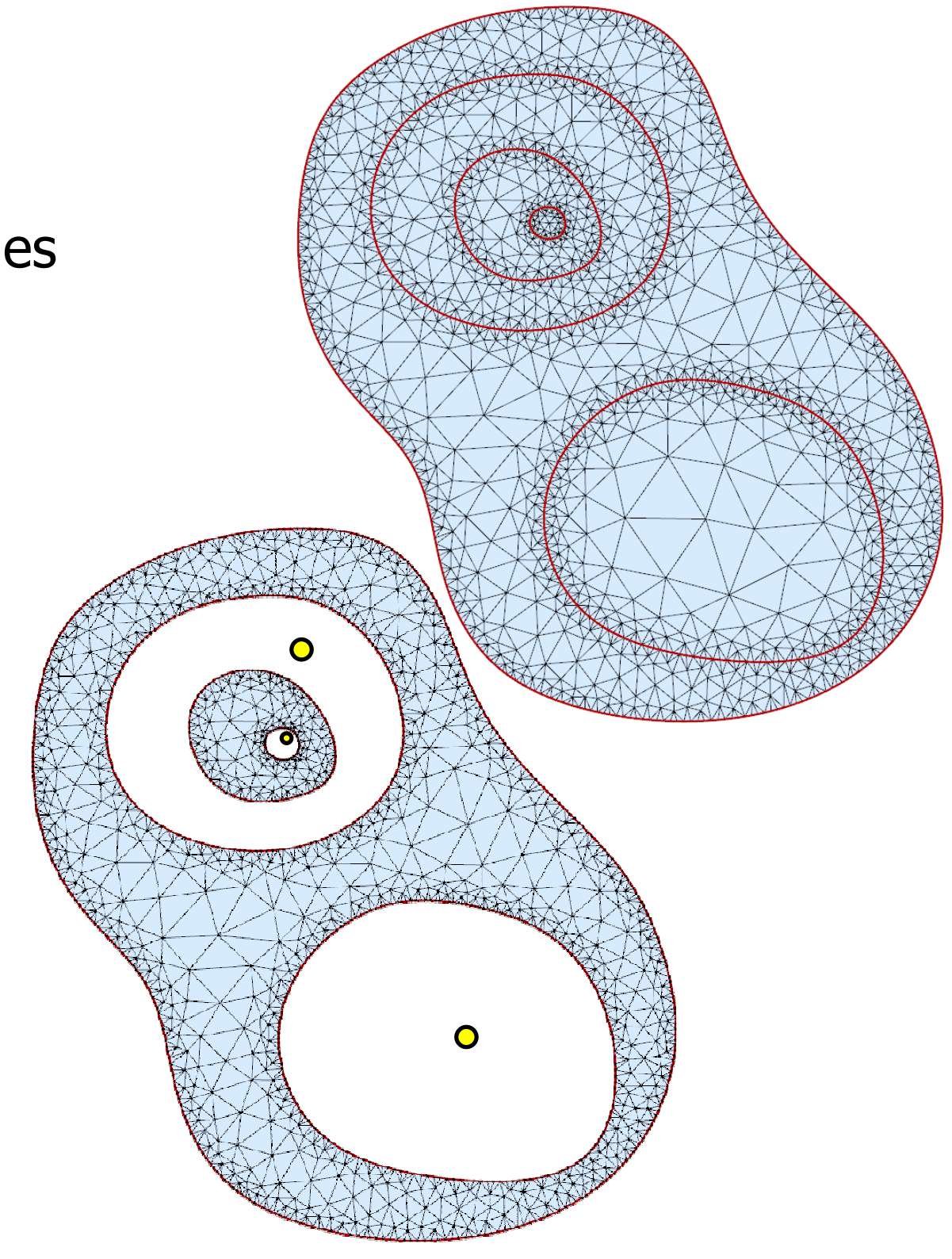




\section{Performances}

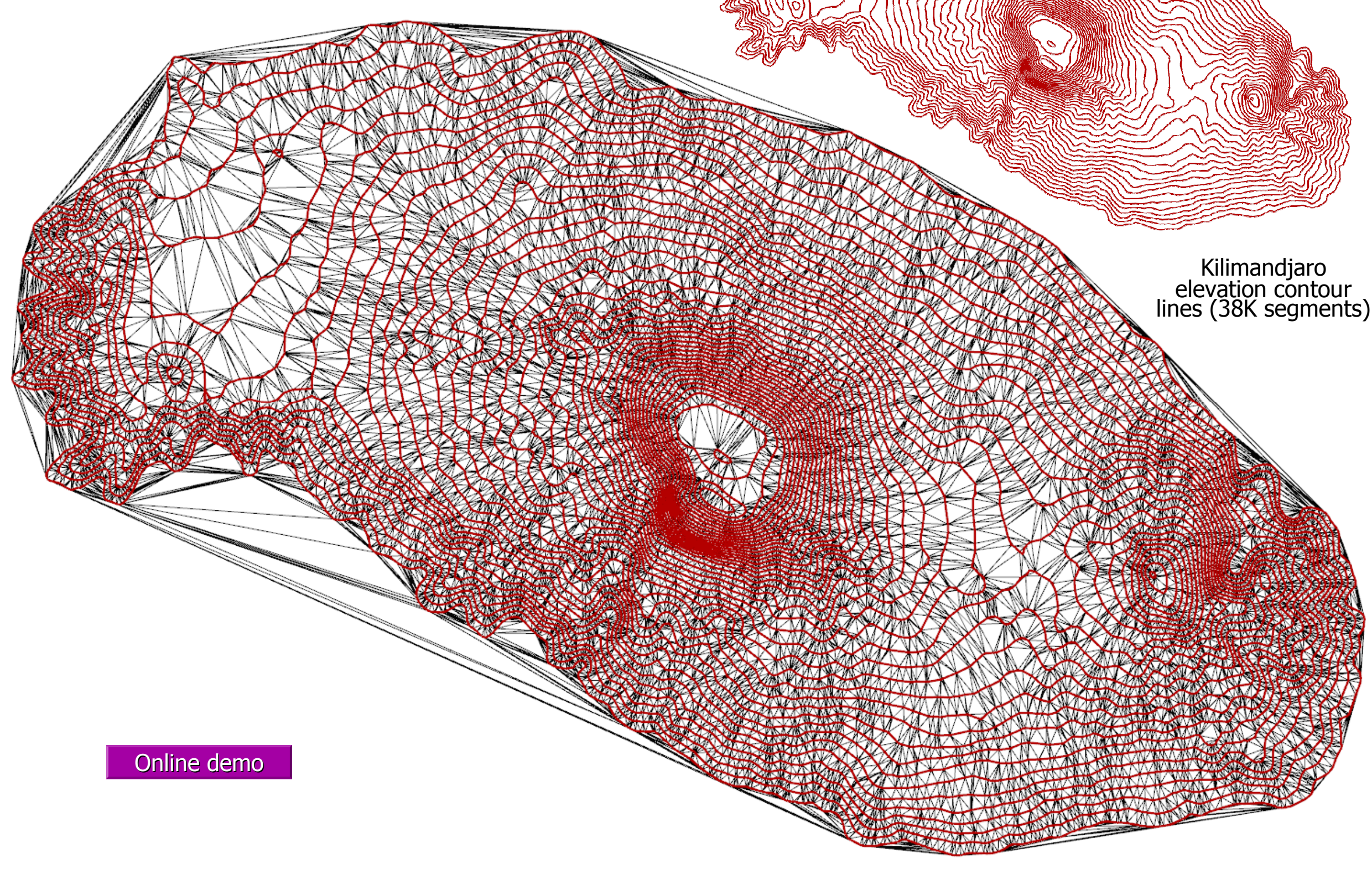




\section{Performances}

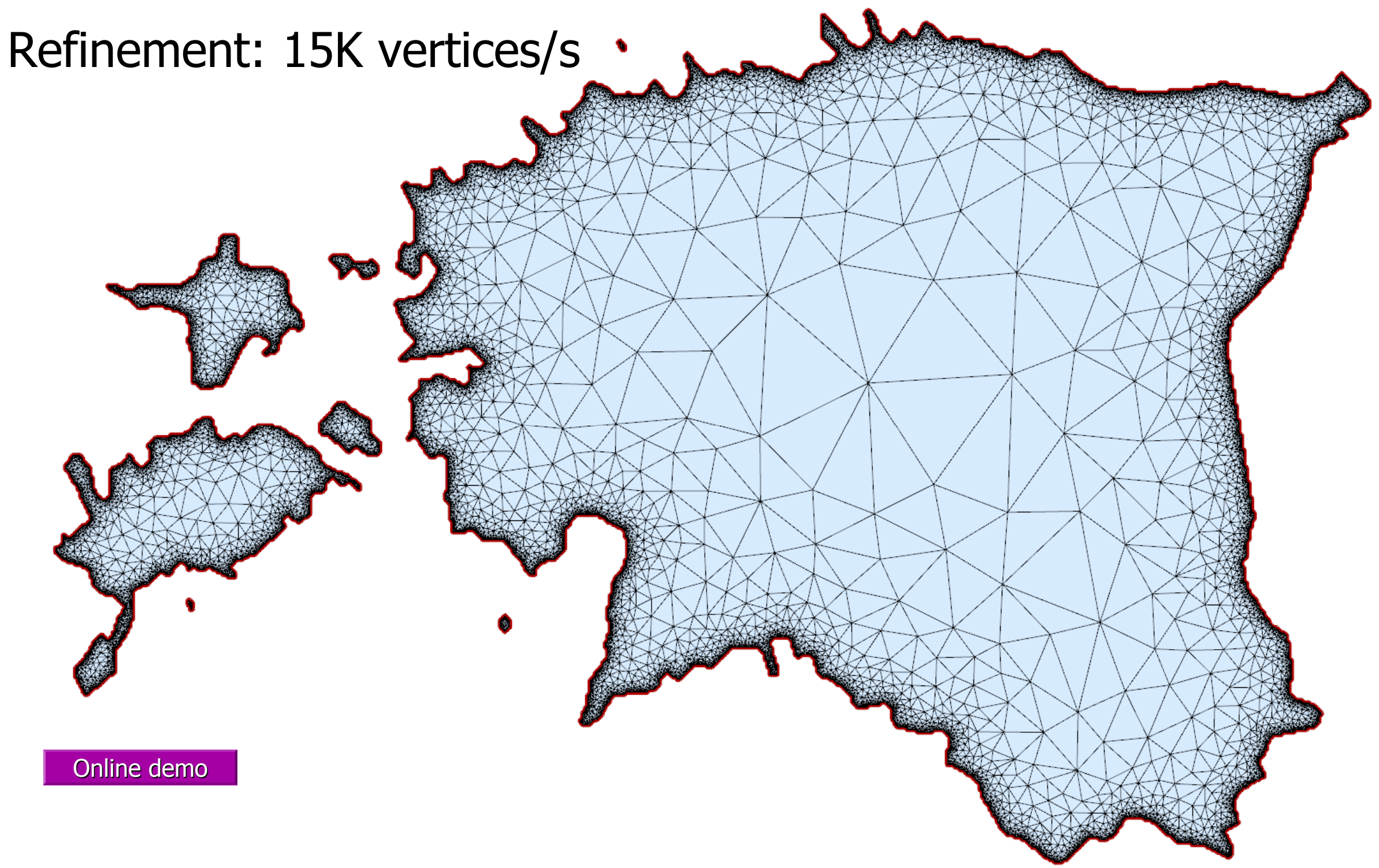




\section{Related Components}

- Voronoi diagram

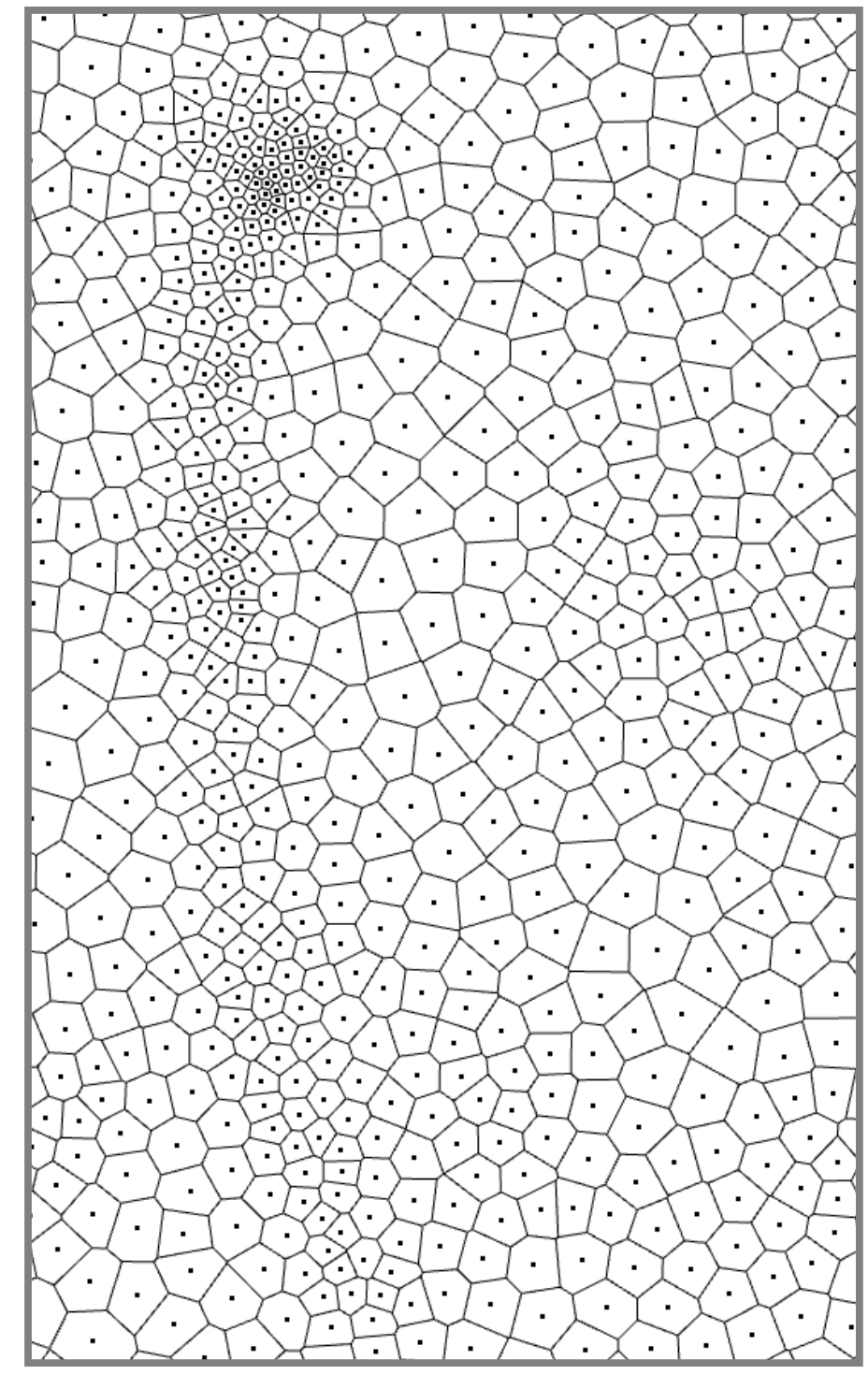




\section{Related Components}

- Voronoi diagram

- Elevation (through traits class)

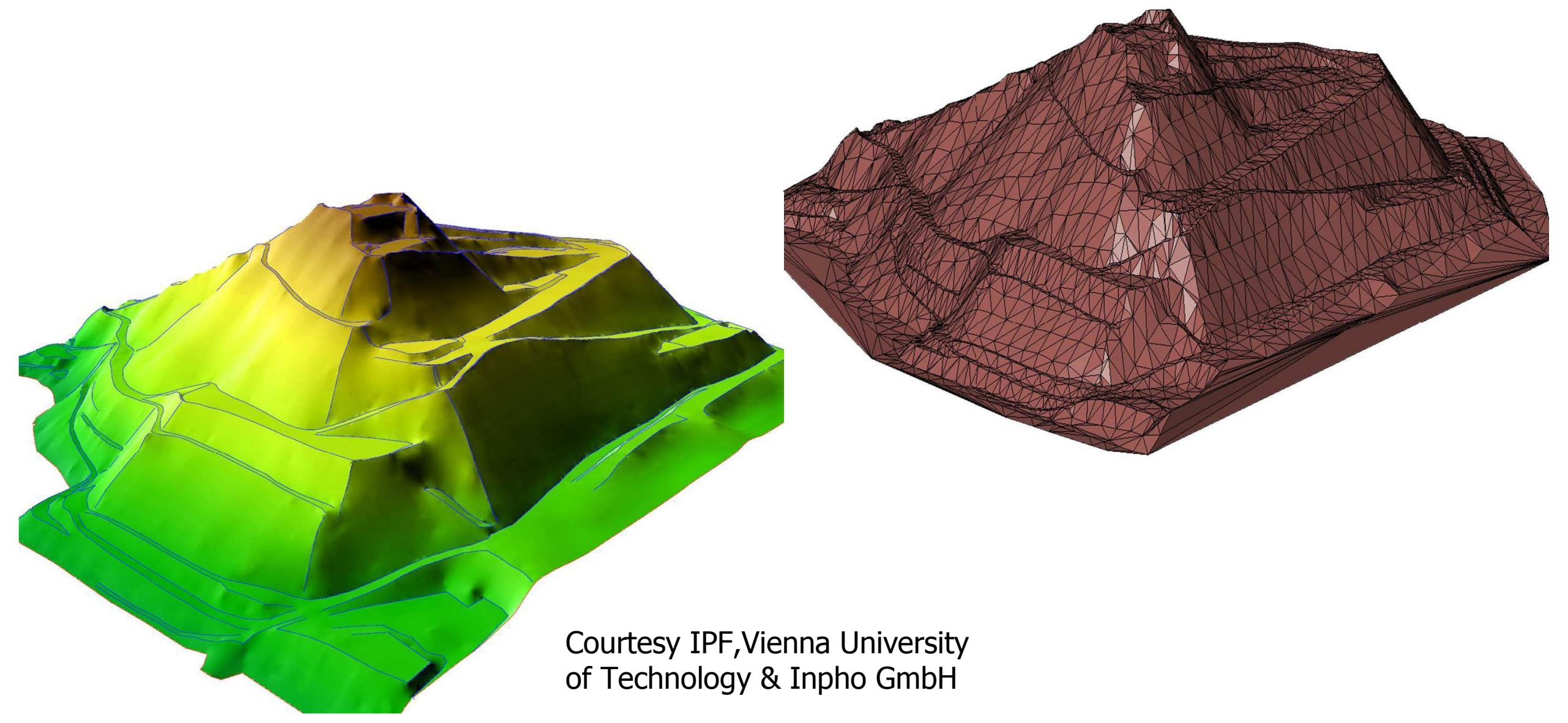




\section{Related Components}

- Voronoi diagram

- Elevation

- Interpolation (natural neighbors)

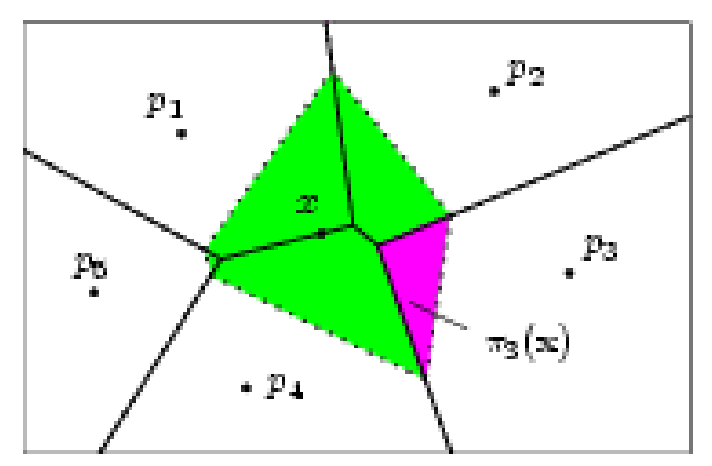




\section{Related Components}

- Voronoi diagram

- Elevation

- Interpolation

- Placement of streamlines
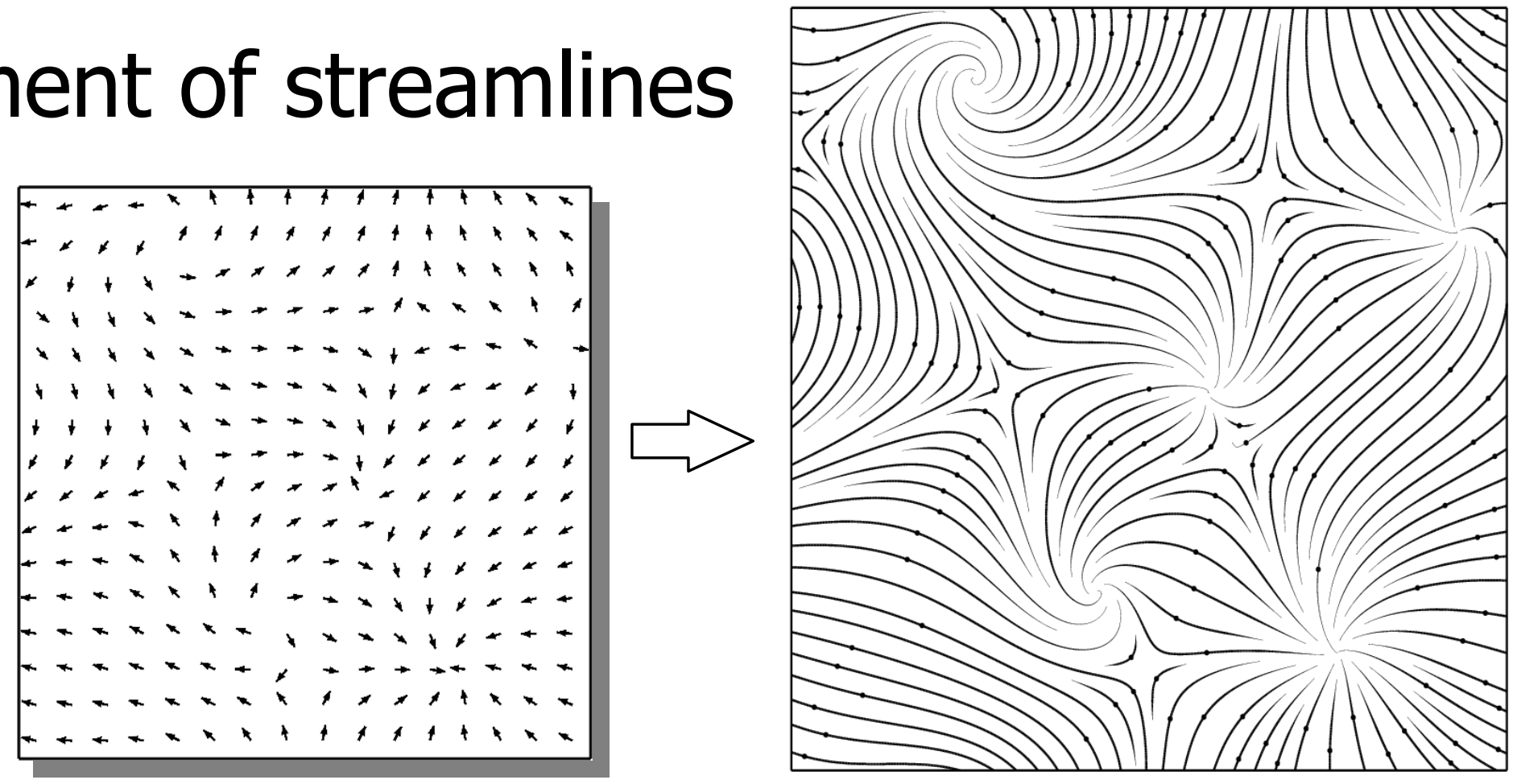
3D 
3D Triangulations

- Delaunay

- Regular

- Rich API

- Fully dynamic

- $1 \mathrm{M}$ points in $16 \mathrm{~s}$

Online manual

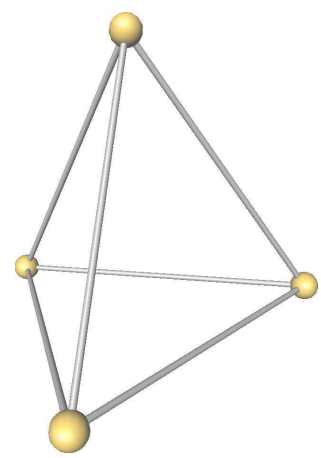

Tetrahedron

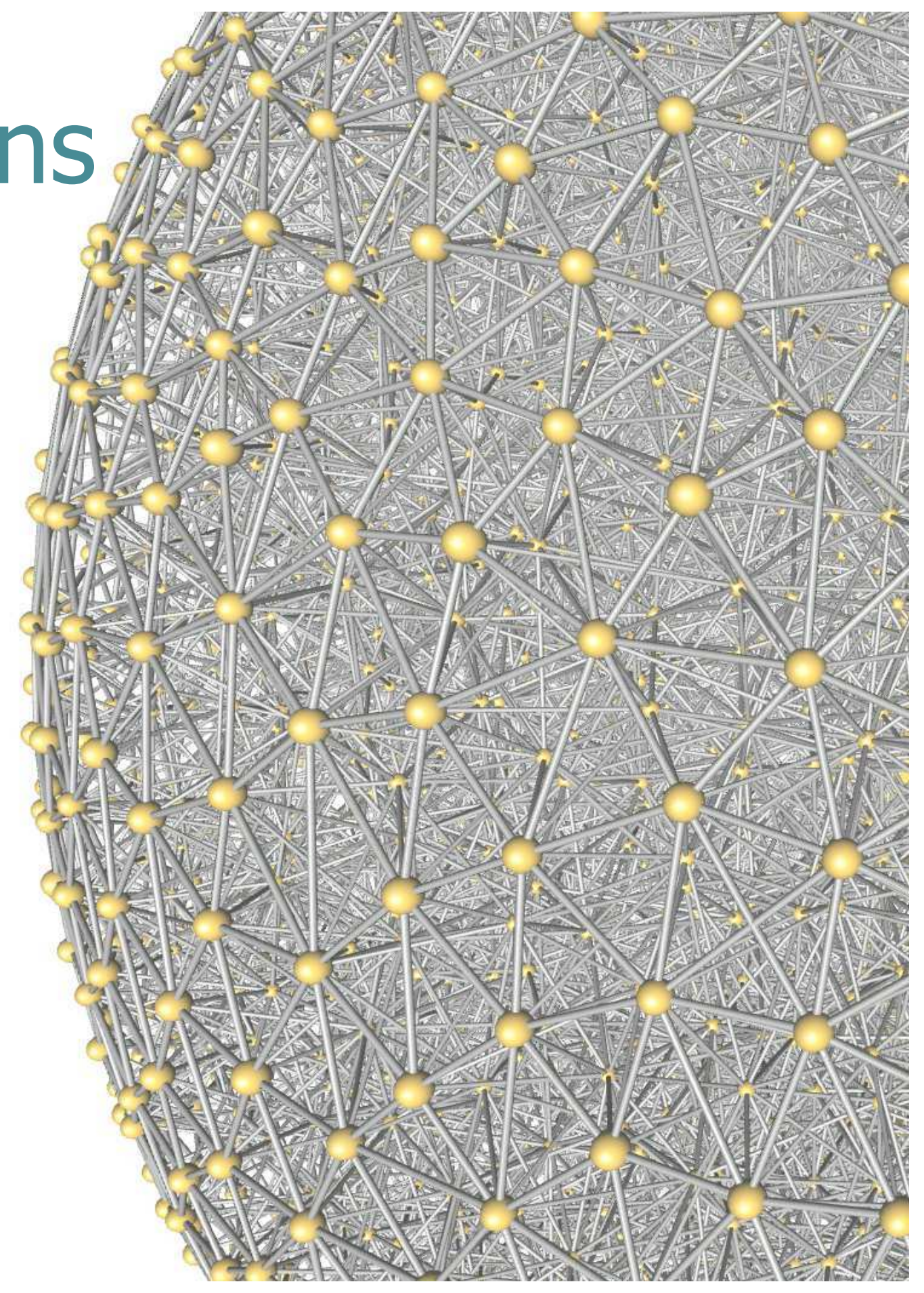




\section{Mesh Generation}

Key concepts:

- Delaunay filtering

- Delaunay refinement
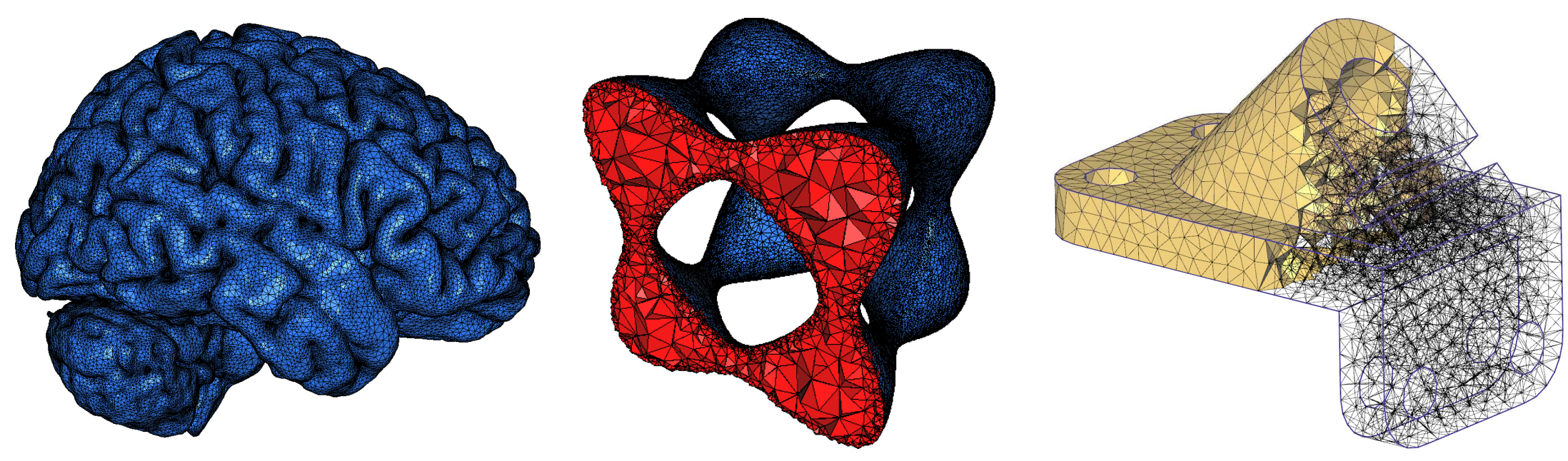


\section{Delaunay Filtering}

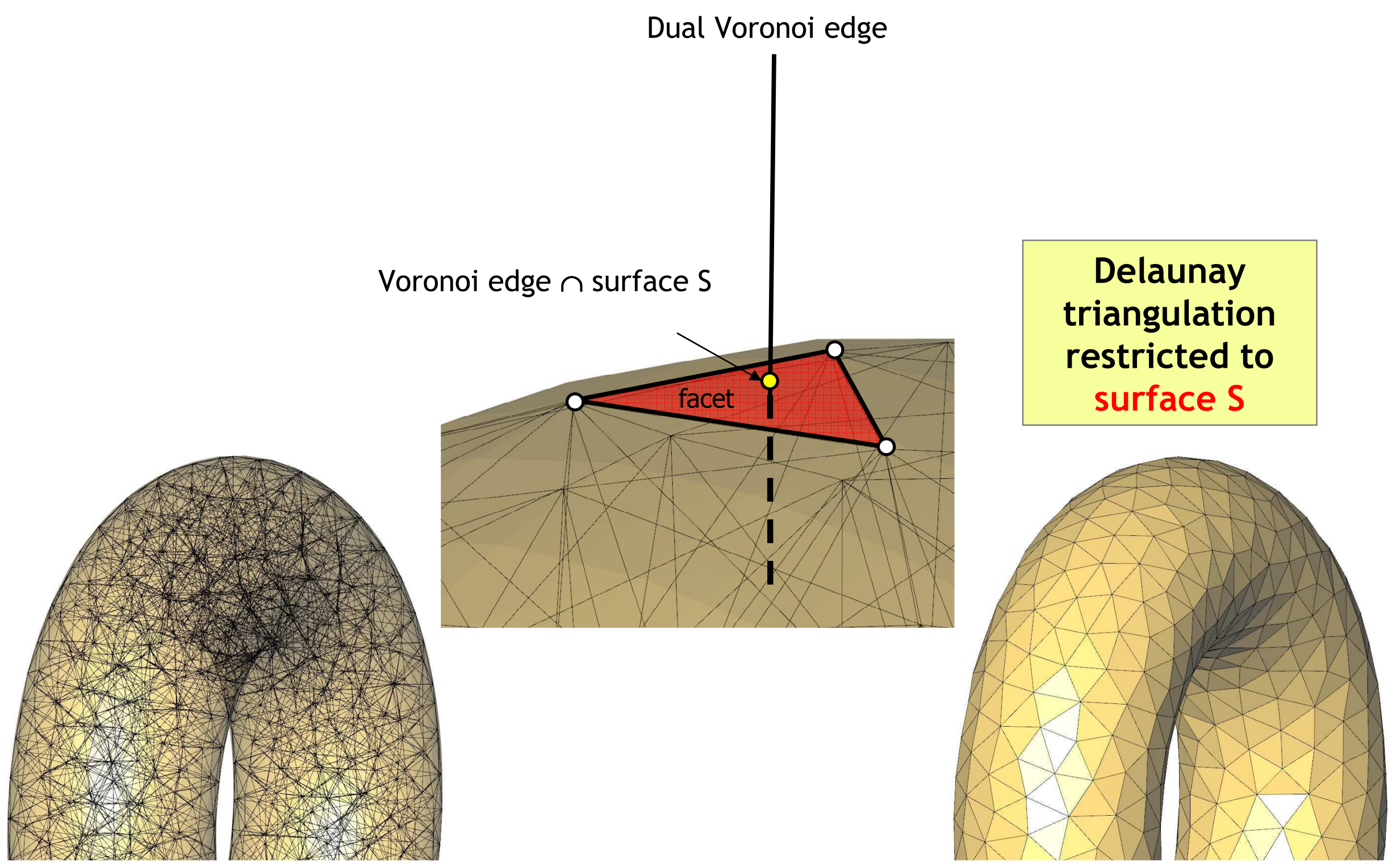




\section{Delaunay Refinement}

Steiner point $\circ$

Bad facet $=$ big or badly shaped or large approximation error

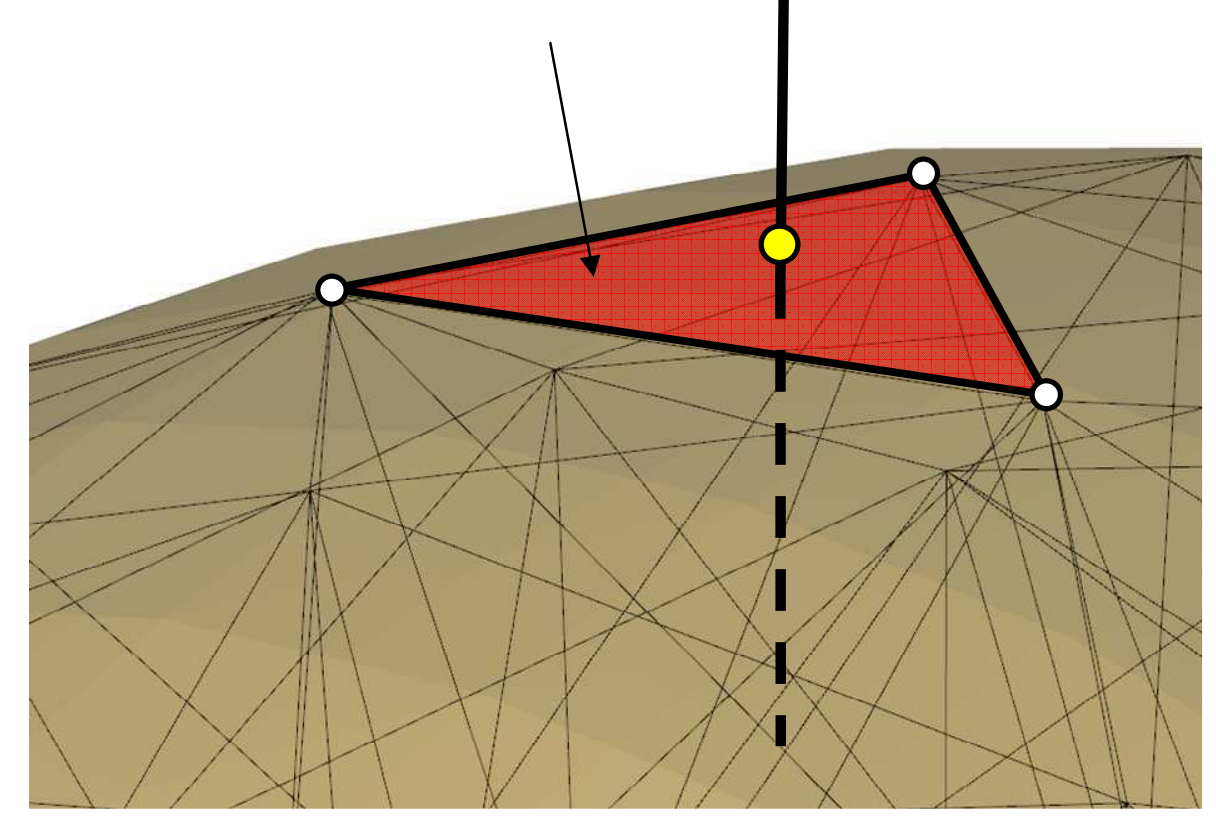




\section{Surface Mesh Generation Algorithm}

\section{repeat}

\{

pick bad facet $\mathbf{f}$

insert furthest (dual(f) $\cap \mathbf{S}$ ) in Delaunay triangulation

update Delaunay triangulation restricted to $\mathbf{S}$

\}

until all facets are good 


\section{Isosurface from 3D Grey Level Image}

\#include <CGAL/Surface_mesh_default_triangulation_3.h>

\#include <CGAL/Complex_2_in_triangulation_3.h>

\#include <CGAL/make_surface_mesh.h>

\#include <CGAL/Gray_level_image_3.h>

\#include <CGAL/Implicit_surface_3.h>

typedef CGAL::Surface_mesh_default_triangulation_3 Tr;

typedef CGAL: :Complex_2_in_triangulation_3<Tr> C2t3;

typedef CGAL::Implicit_surface_3<Kernel, Gray_level_image> Surface_3;

Tr tr; // 3D-Delaunay triangulation

C2t3 c2t3 (tr); // 2D-complex in 3D-Delaunay triangulation

Gray_level_image image ("data/brain", 128);

Surface_3 surface (image, bounding_sphere, 1e-2);

// Criteria: min triangle angles, size, approximation error,

CGAL : :Surface_mesh_default_criteria_3<Tr> criteria $(30,5 ., 5$.$) ;$

CGAL : :make_surface_mesh(c2t3, surface, criteria, CGAL: :Manifold_tag()); 


\section{Output Mesh}

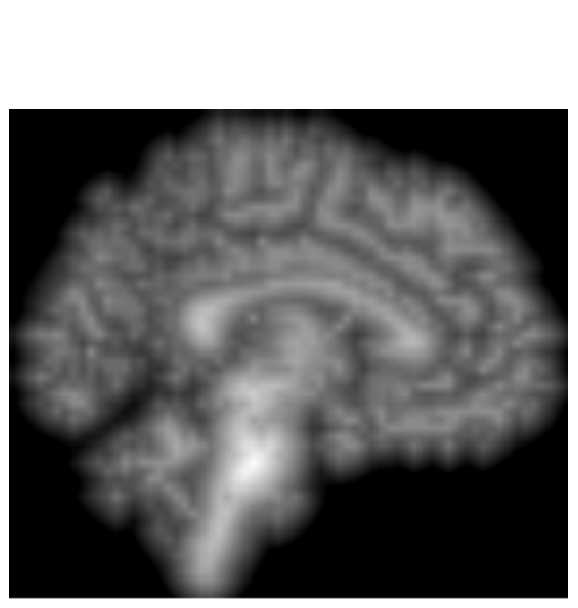

input

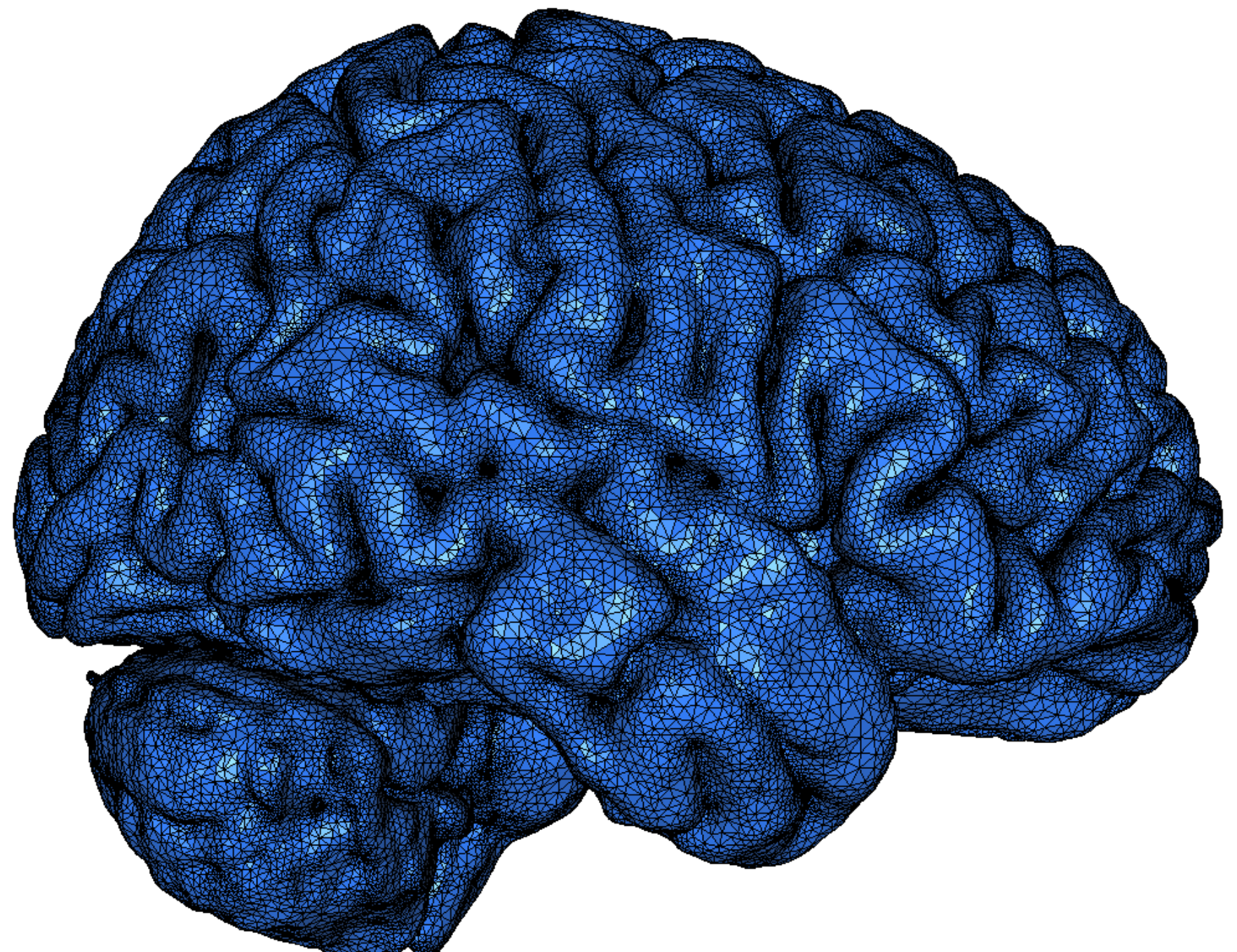

Triangle surface mesh approximating $\mathrm{S}$ 


\section{Output Mesh Properties}

- Well shaped triangles

- Lower bound on triangle angles

- Homeomorphic to input surface

- Manifold

- not only combinatorially, i.e., no self-intersection

- Faithful Approximation of input surface

- Hausdorff distance

- Normals

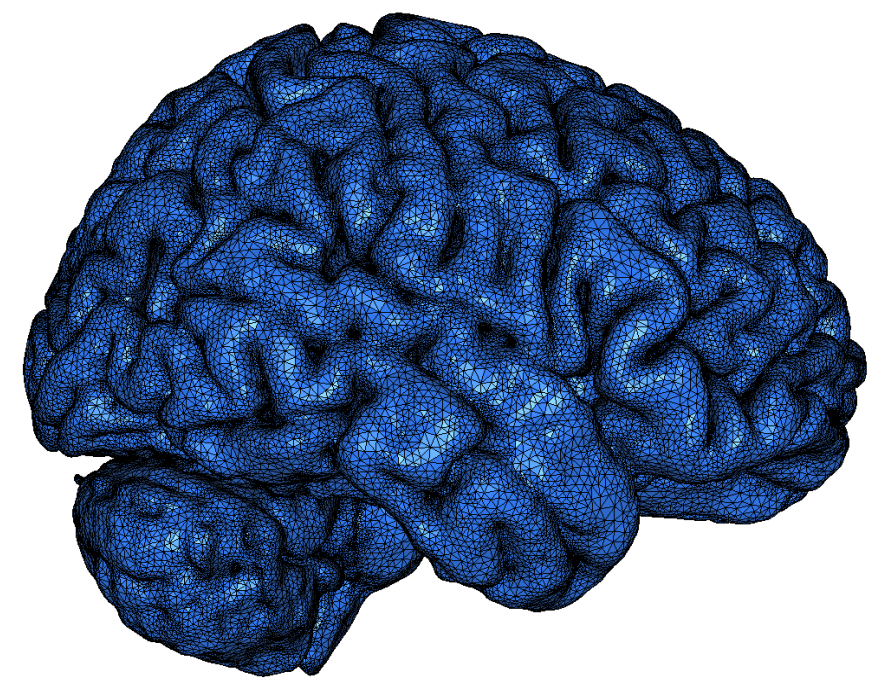




\section{Delaunay Refinement vs Marching Cubes}

Delaunay refinement

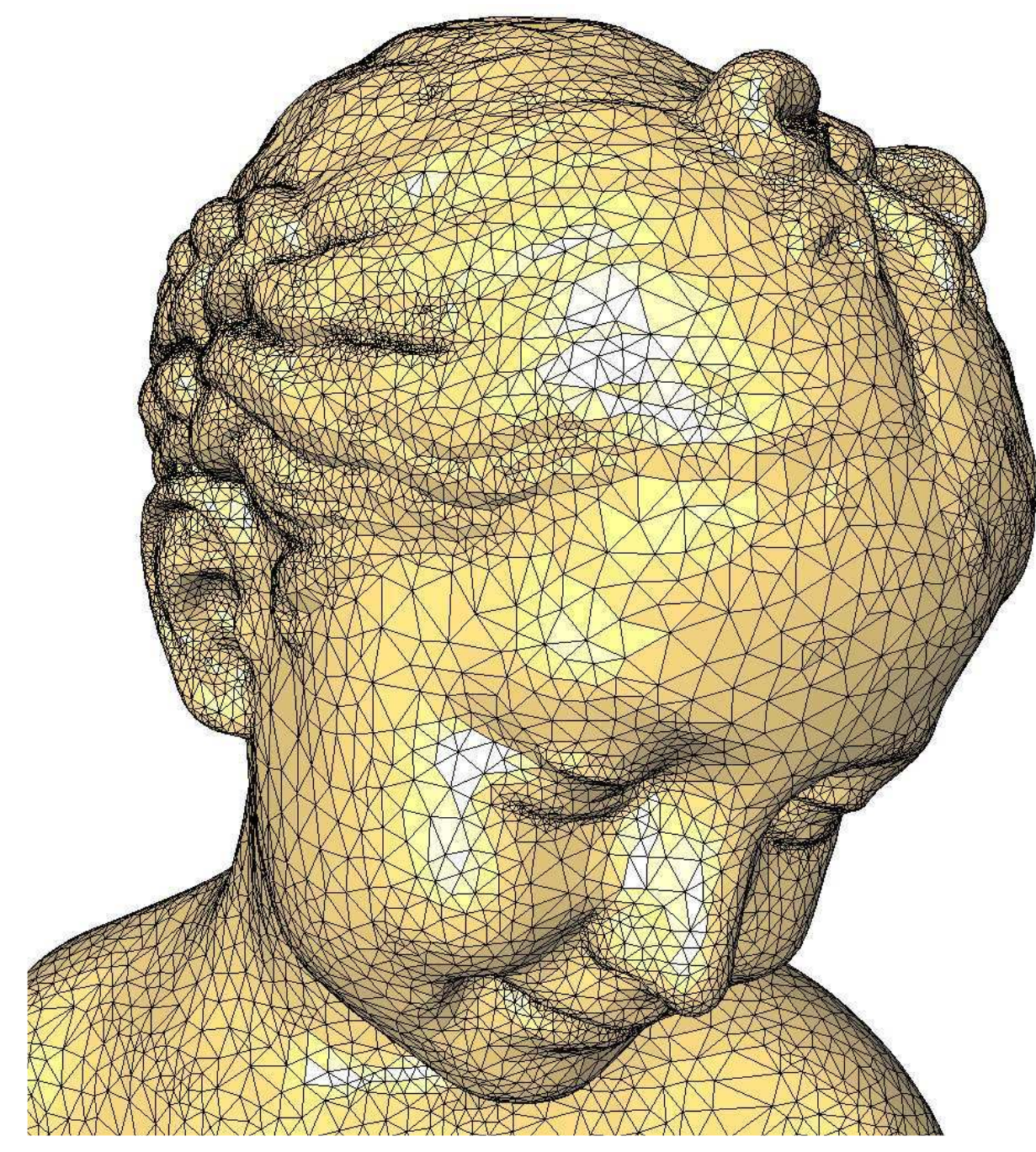

Marching cubes in octree

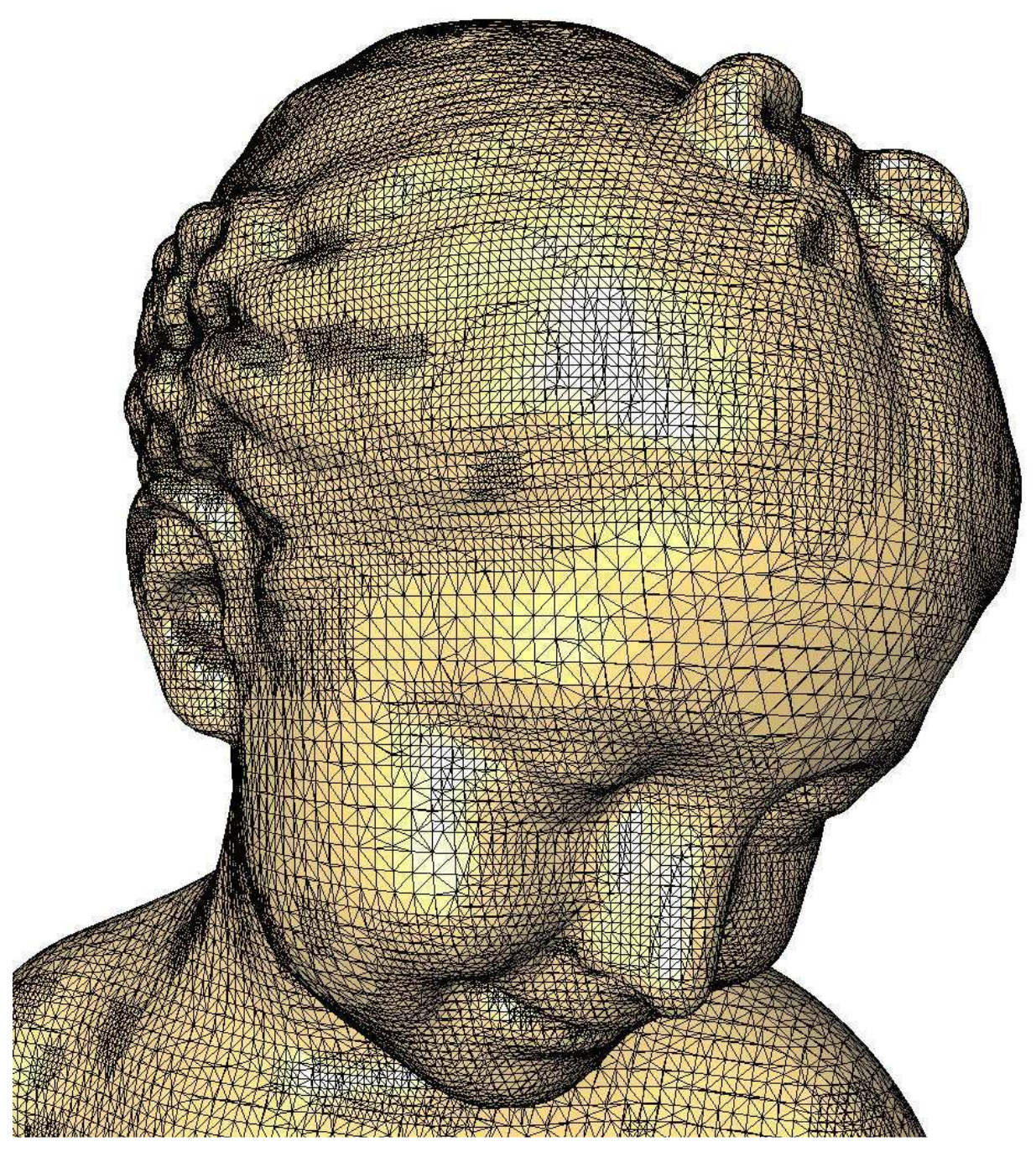




\section{Surface Remeshing}

\section{Input is a polyhedral surface}

(requires efficient data structures for intersection computations)

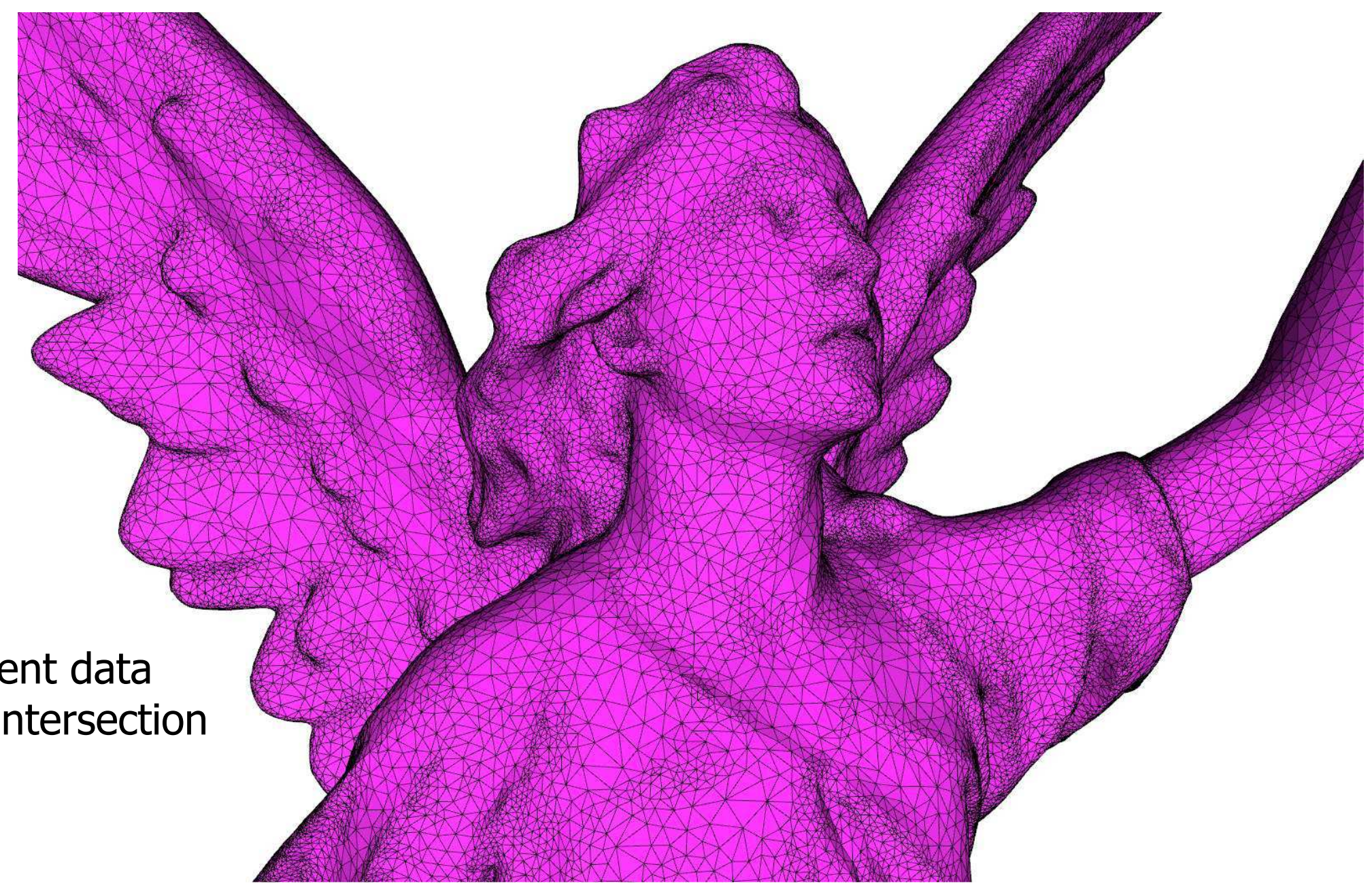




\section{Parameters}

- Shape of triangles

- lower bound on triangle angles

- Size 


\section{Parameters}

- Shape of triangles

- lower bound on triangle angles

- Size

- No constraint 


\section{Parameters}

- Shape of triangles

- lower bound on triangle angles

- Size

- No constraint

- Uniform sizing 


\section{Parameters}

- Shape of triangles

- lower bound on triangle angles

- Size

- No constraint

- Uniform sizing

- Sizing function 


\section{Parameters}

- Shape of triangles

- lower bound on triangle angles

- Size

- No constraint

- Uniform sizing

- Sizing function

- Approximation error

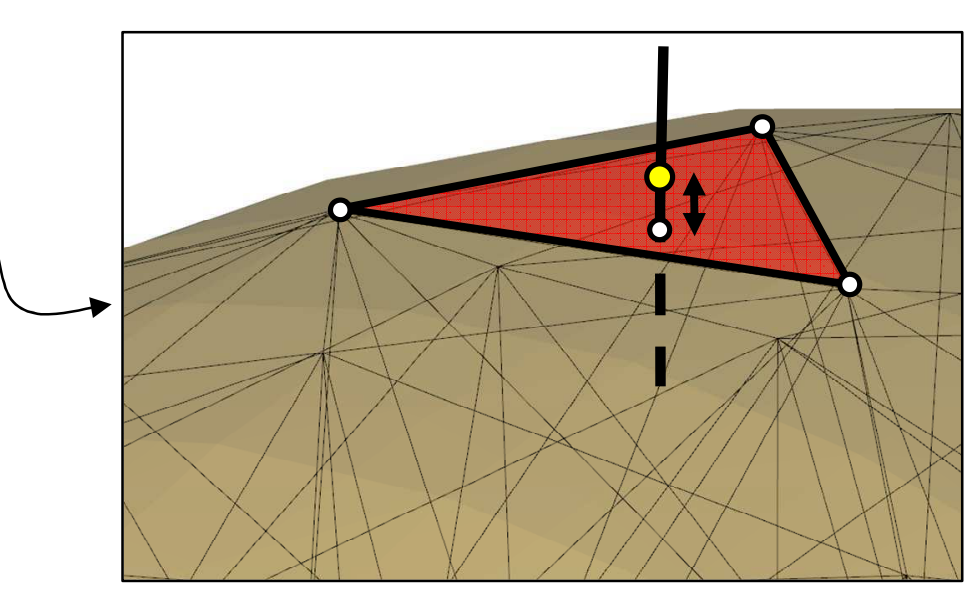




\section{Uniform vs Adapted}

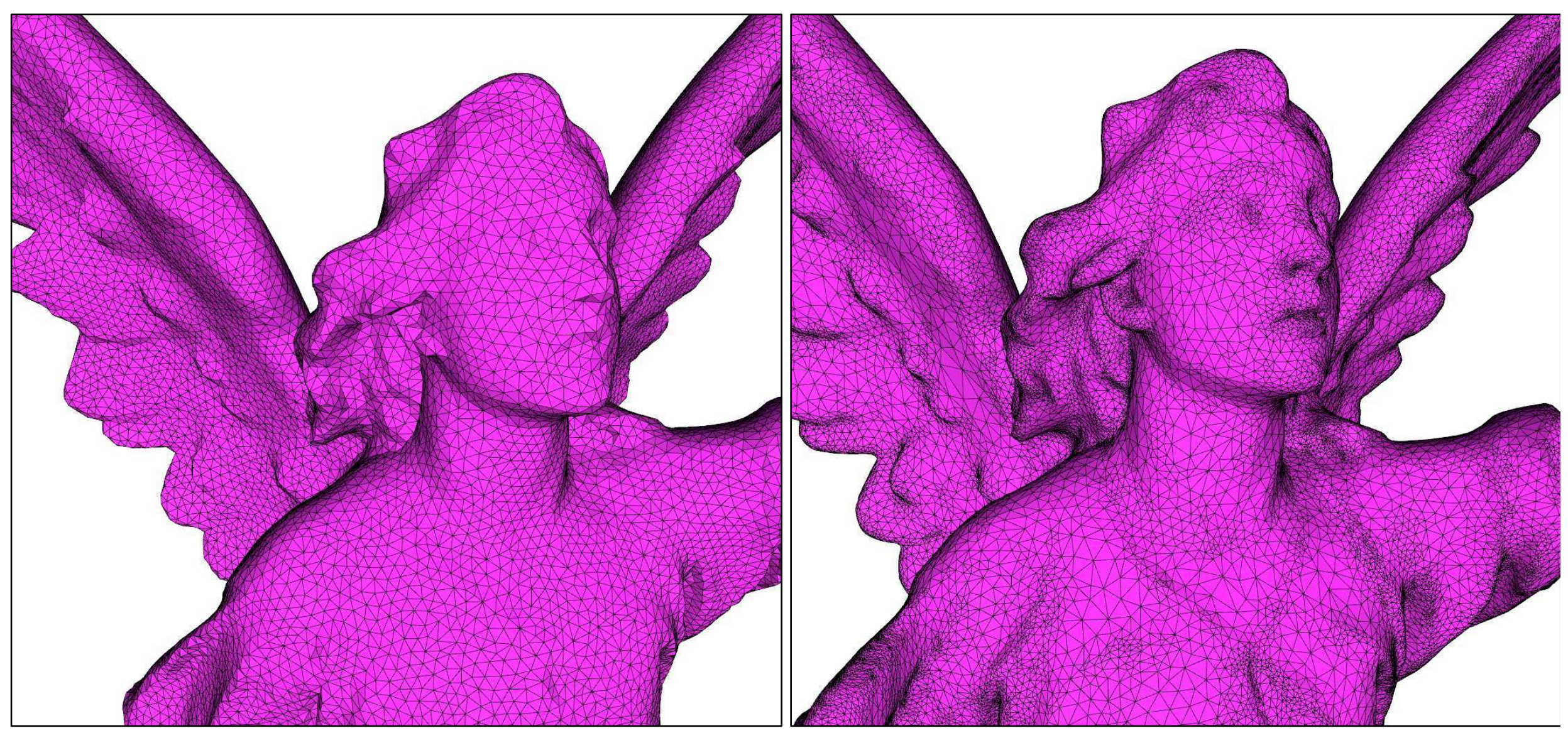




\section{Mesh Generation Framework}

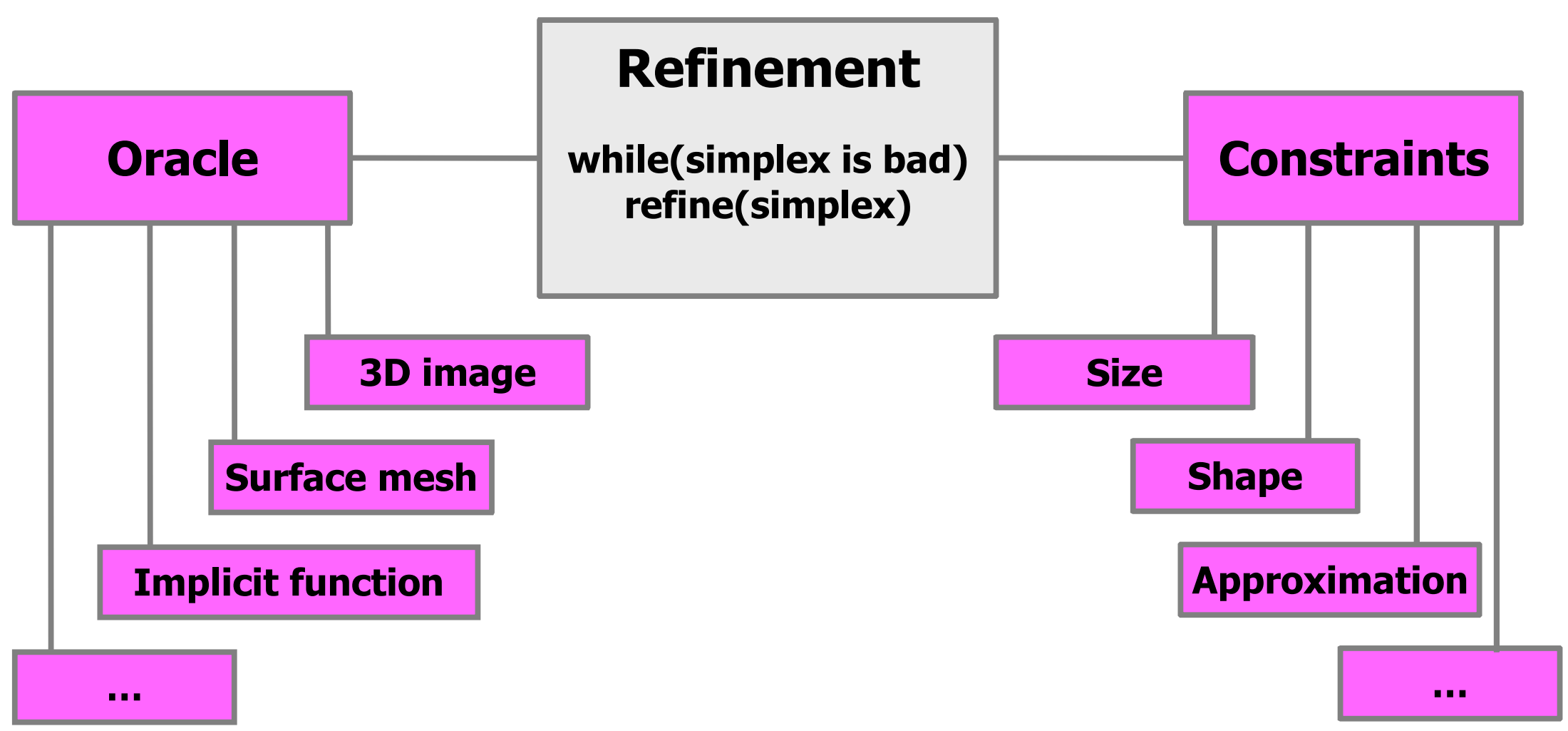




\section{A Versatile Framework}

- 3D grey level images

- 3D multi-domain images

- Implicit function: $f(x, y, z)=$ constant

- Surface mesh (remeshing)

- Point set (surface reconstruction)

- Anything which provides intersections 


\section{Next Release}


Volume Mesh Generation
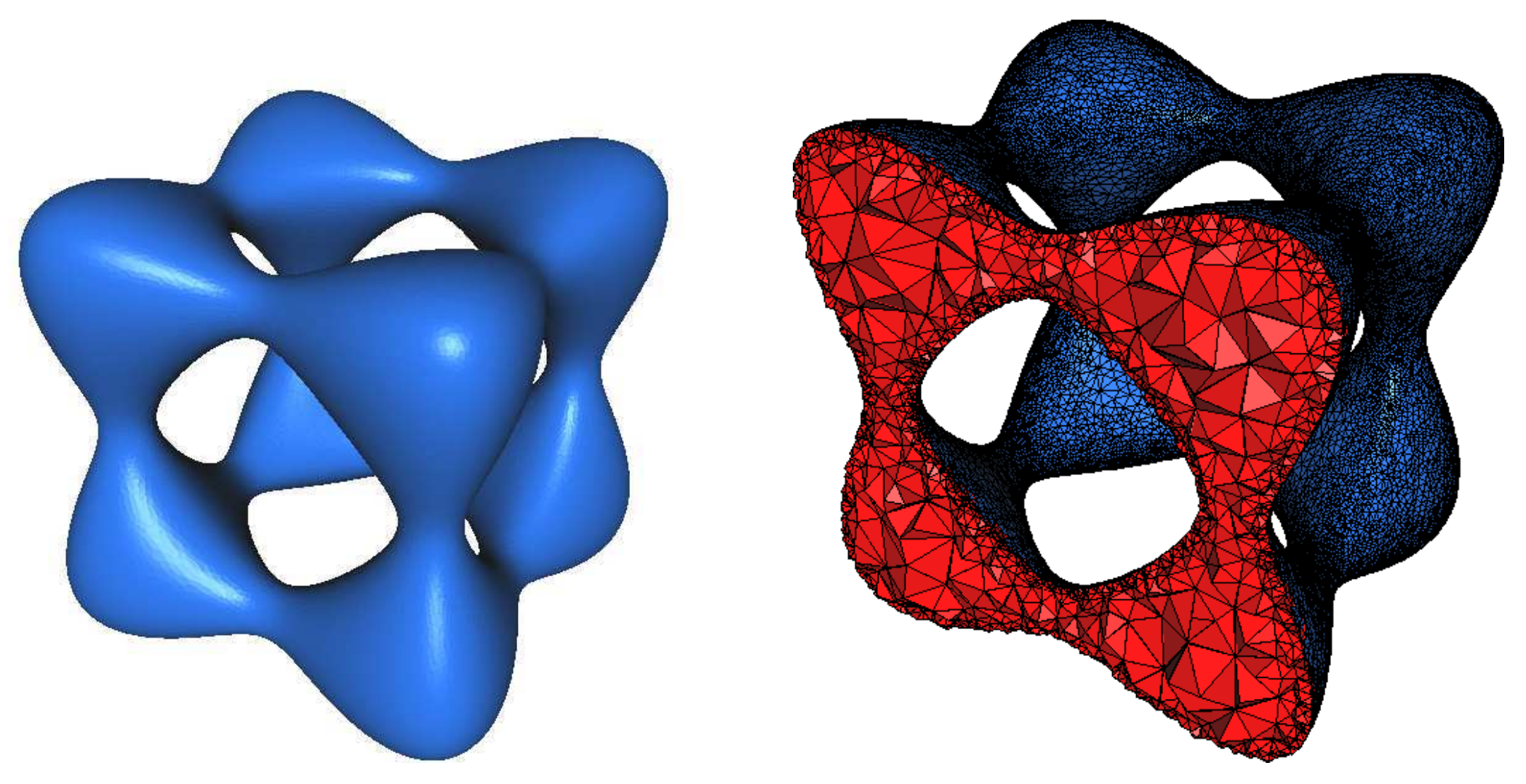


\section{More Delaunay Filtering}

Delaunay
triangulation
restricted to
domain $\Omega$

tetrahedron
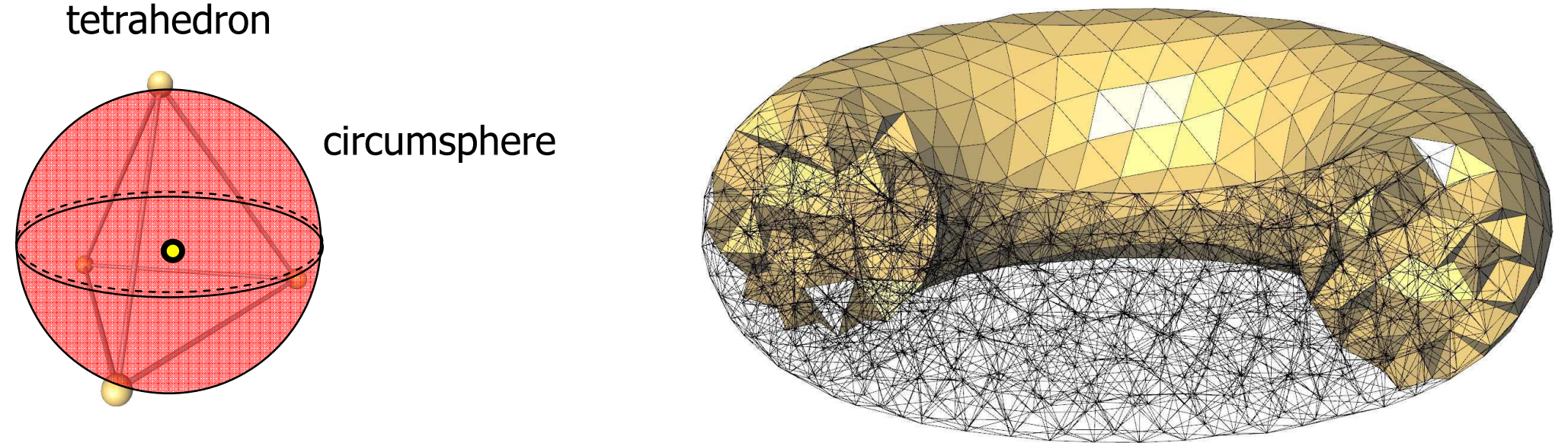

Dual Voronoi vertex inside domain $\Omega$ ("oracle")

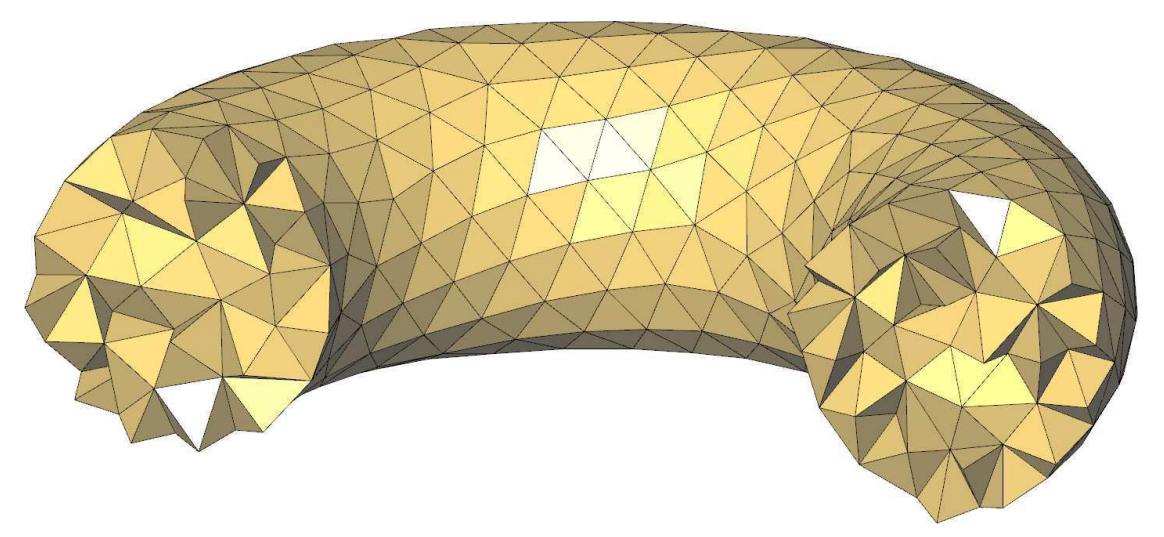




\section{Delaunay Refinement}

Steiner point $\quad 0$

Bad tetrahedron $=$ big or badly shaped

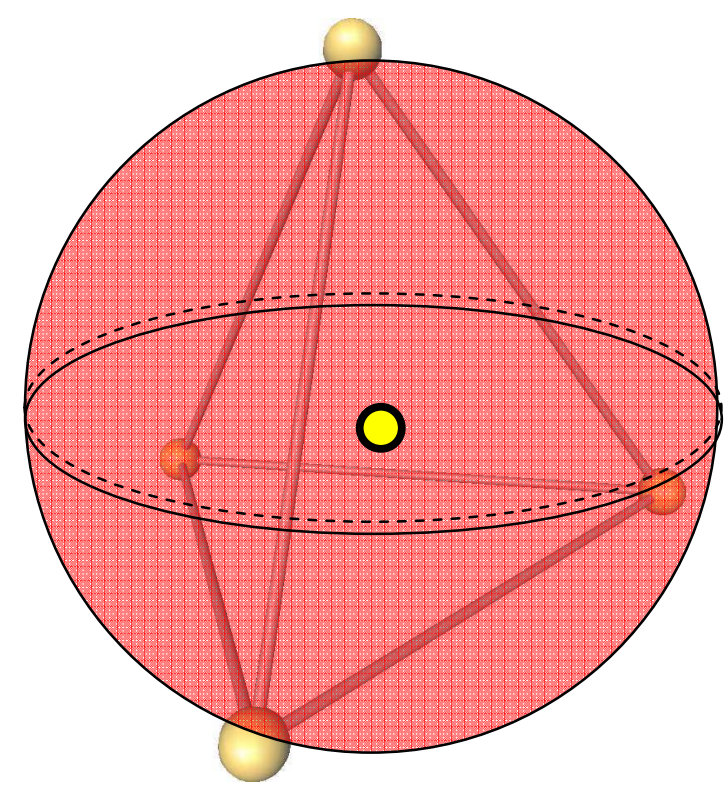




\section{Volume Mesh Generation Algorithm}

repeat

\{

pick bad simplex

if(Steiner point encroaches a facet)

refine facet

else

refine simplex

update Delaunay triangulation restricted to domain

\}

until all simplices are good

Exude slivers 


\section{Tetrahedron Zoo}
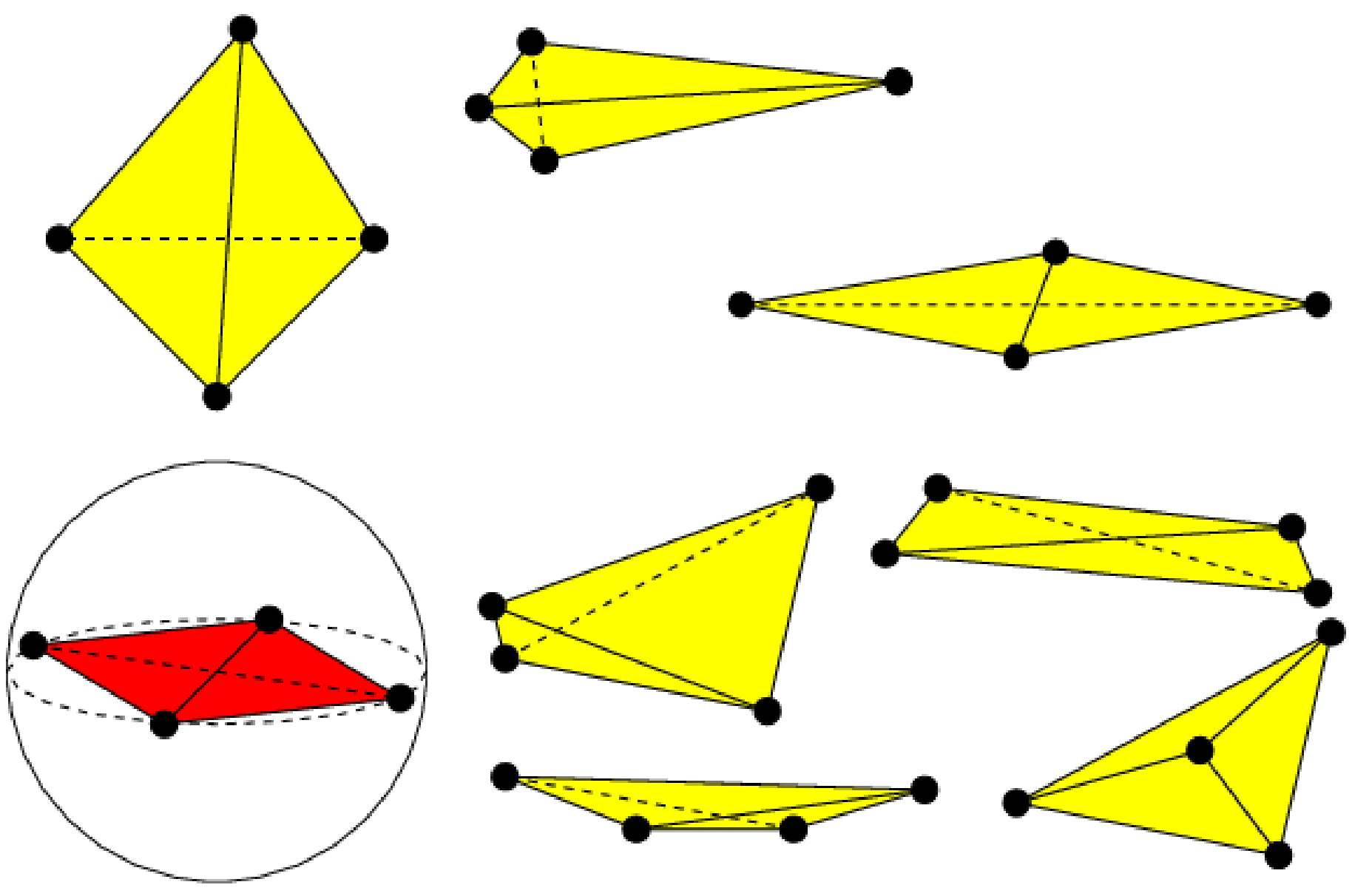

sliver 


\section{Sliver Exudation [Edelsbrunner-Guoy]}

- Delaunay triangulation turned into a regular triangulation with null weights.

- Small increase of weights triggers edgefacets flips to remove slivers.

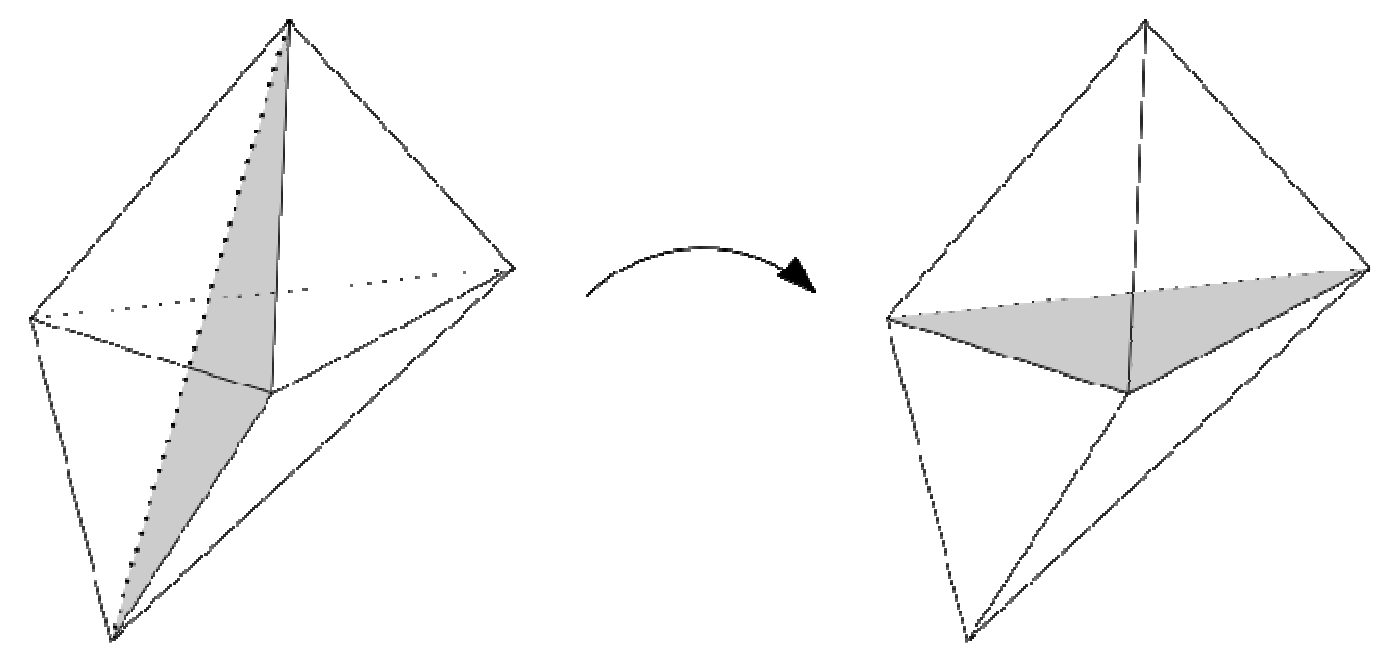




\section{Sliver Exudation Process}

- Try improving all tetrahedra with an aspect ratio lower than a given bound

- Never flips a boundary facet
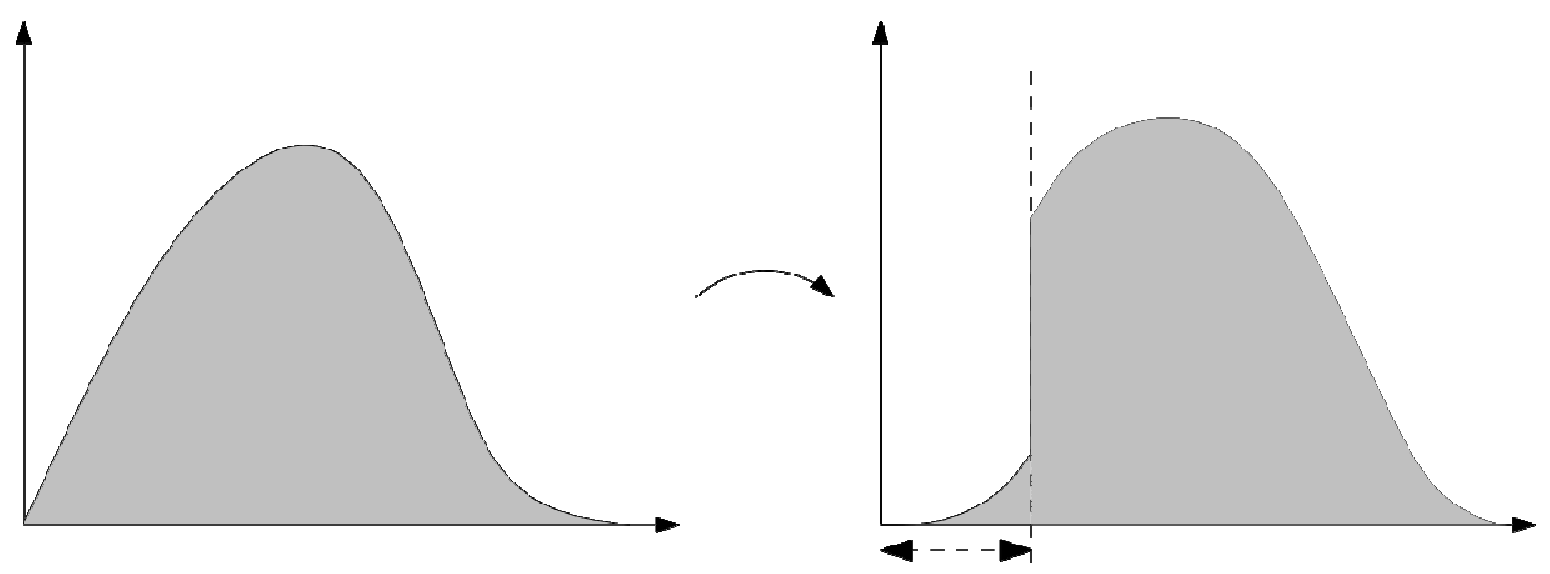

distribution of aspect ratios 


\section{D Mesh from Multi-Domain Images}

Tr tr; // 3D Delaunay triangulation

C2t3 c2t3(tr); // 2D complex in 3D-Delaunay triangulation

Image_3 image ("segmented_visible_human");

Mesh_traits mesh_traits (image);

Facets_criteria facets_criteria(5, 1); // facet sizing

// approximation error

Tets_criteria tets_criteria(5);

// tet sizing

// 0.5 = radius-radius ratio upper bound for sliver exudation

CGAL : :make_mesh_3_for_multivolumes (c2t3, mesh_traits, facets_criteria,

tets_criteria, 0.5); 


\section{Output Volume Mesh}

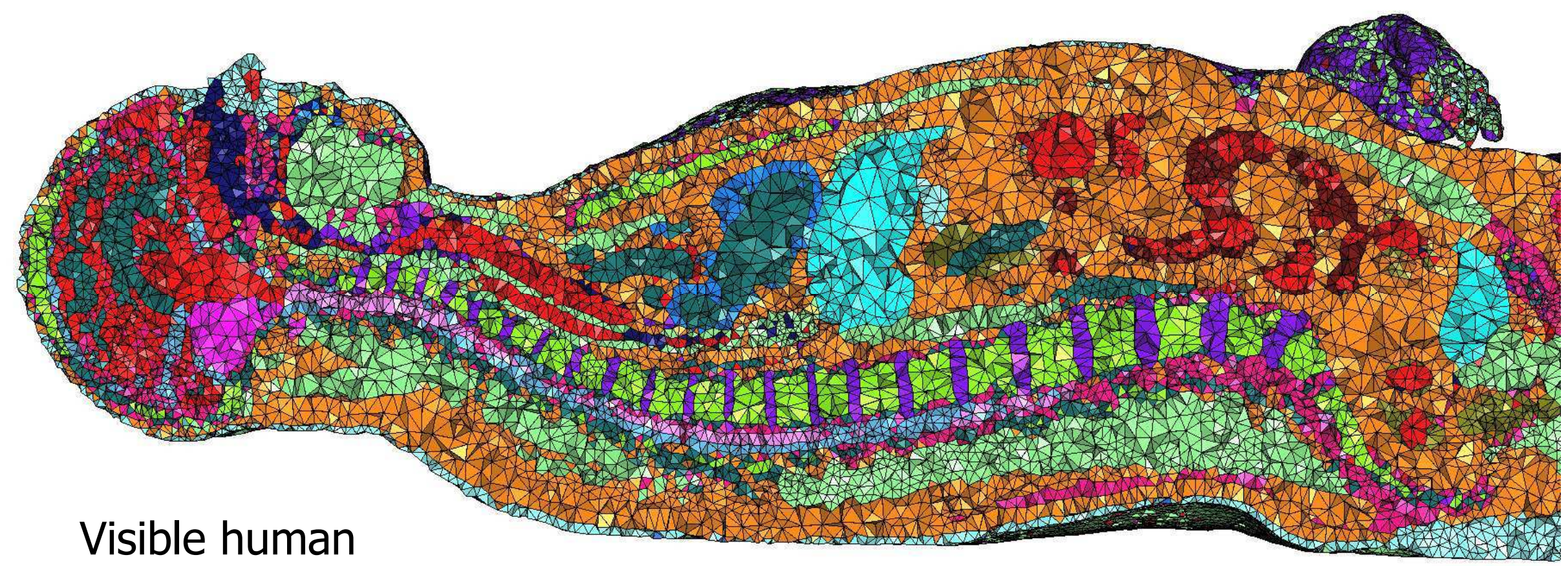




\section{Work in Progress}




\section{Piecewise Smooth Surfaces}
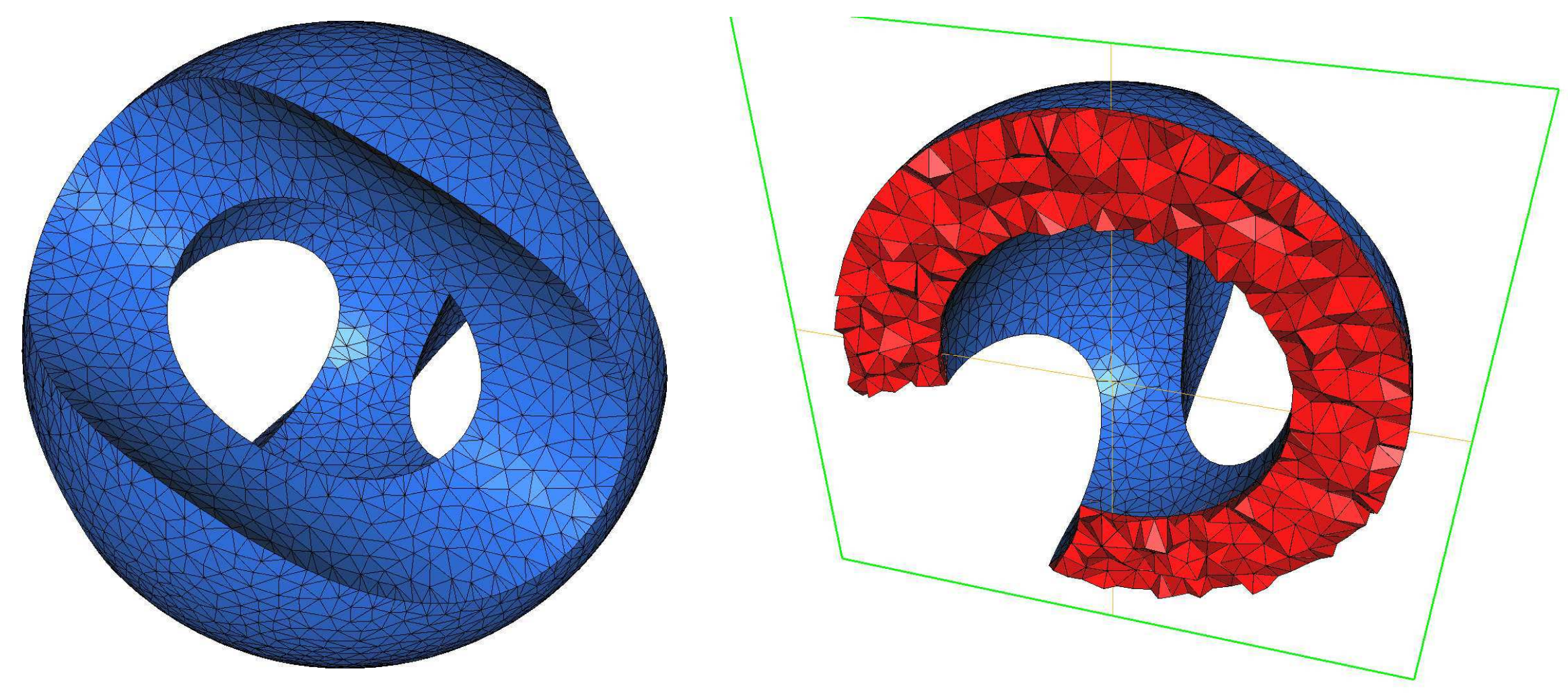


\section{Input: Piecewise smooth complex}

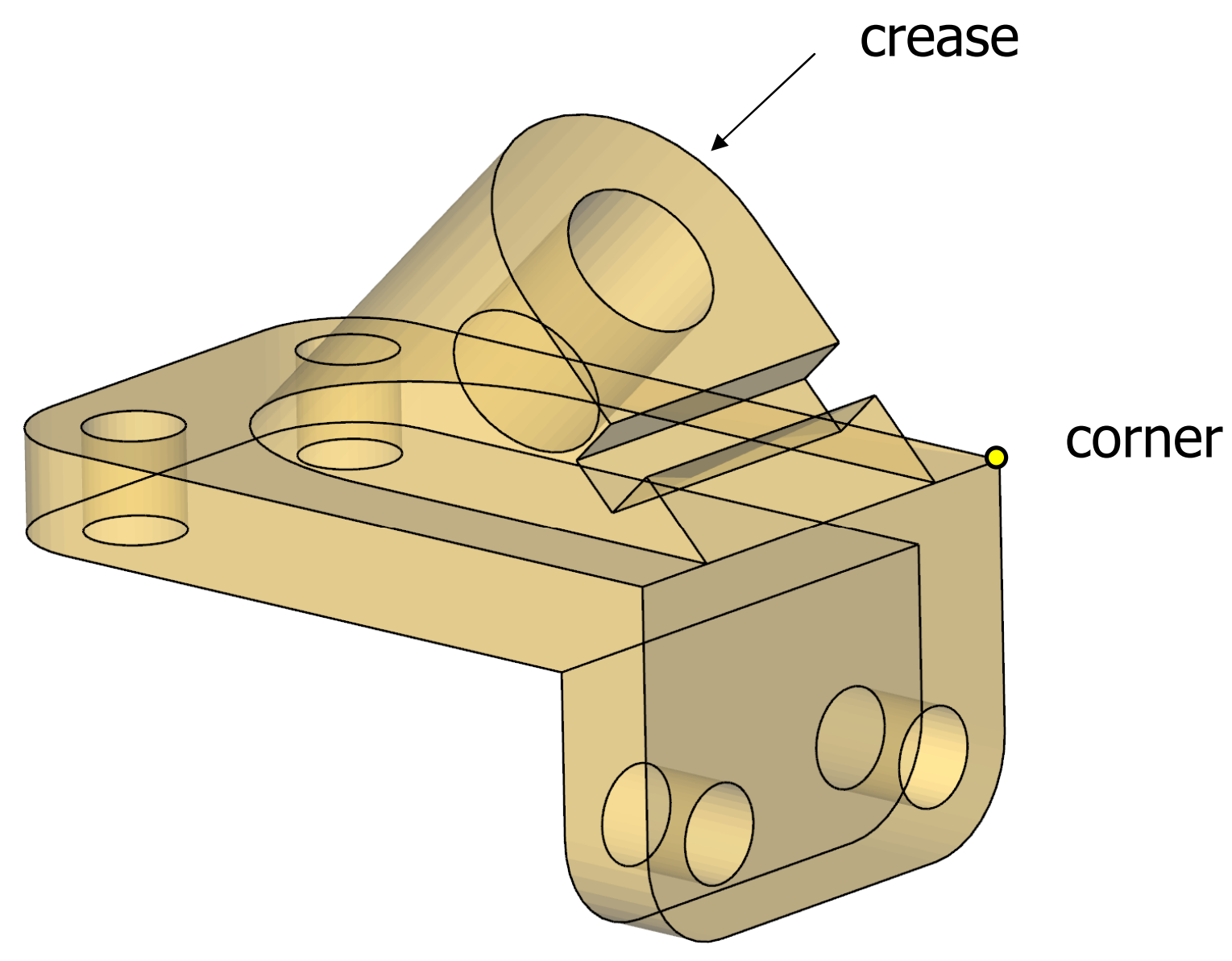




\section{More Delaunay Filtering}

primitive

Voronoi vertex tetrahedron

Voronoi edge facet

Voronoi face edge

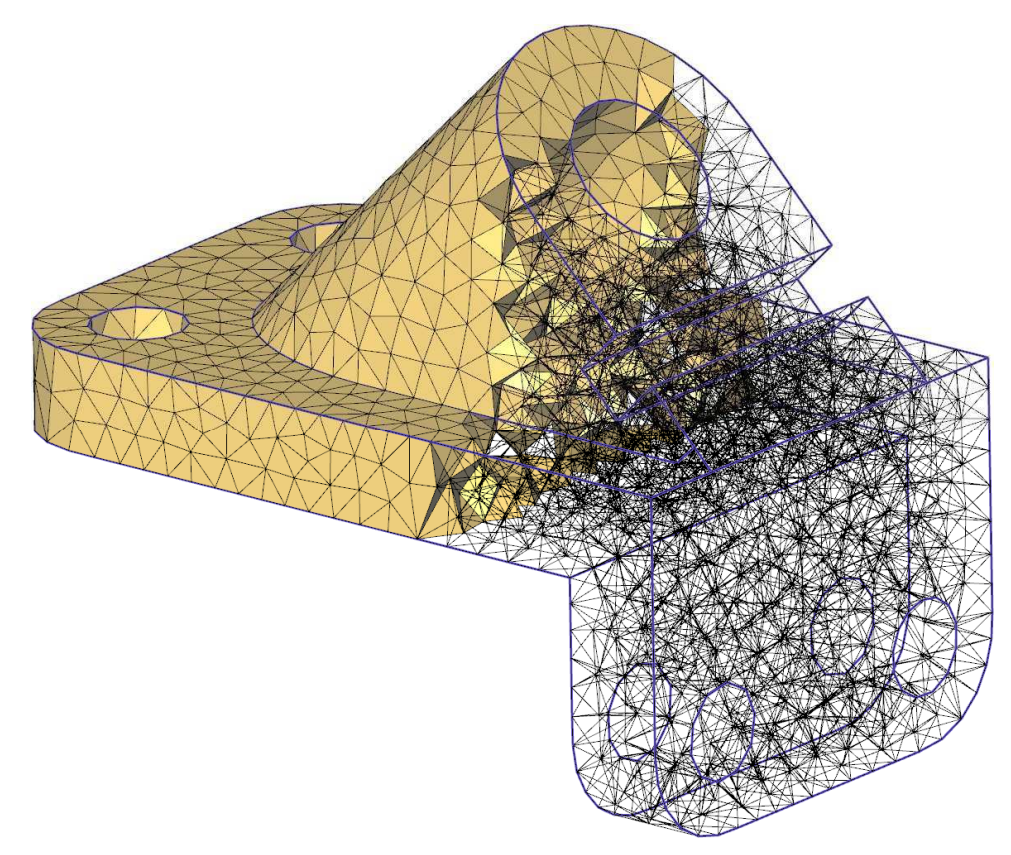

test

inside

intersect domain boundary

intersect crease against

domain

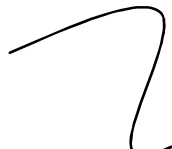




\section{Delaunay Refinement}

- Steiner points $\circ$
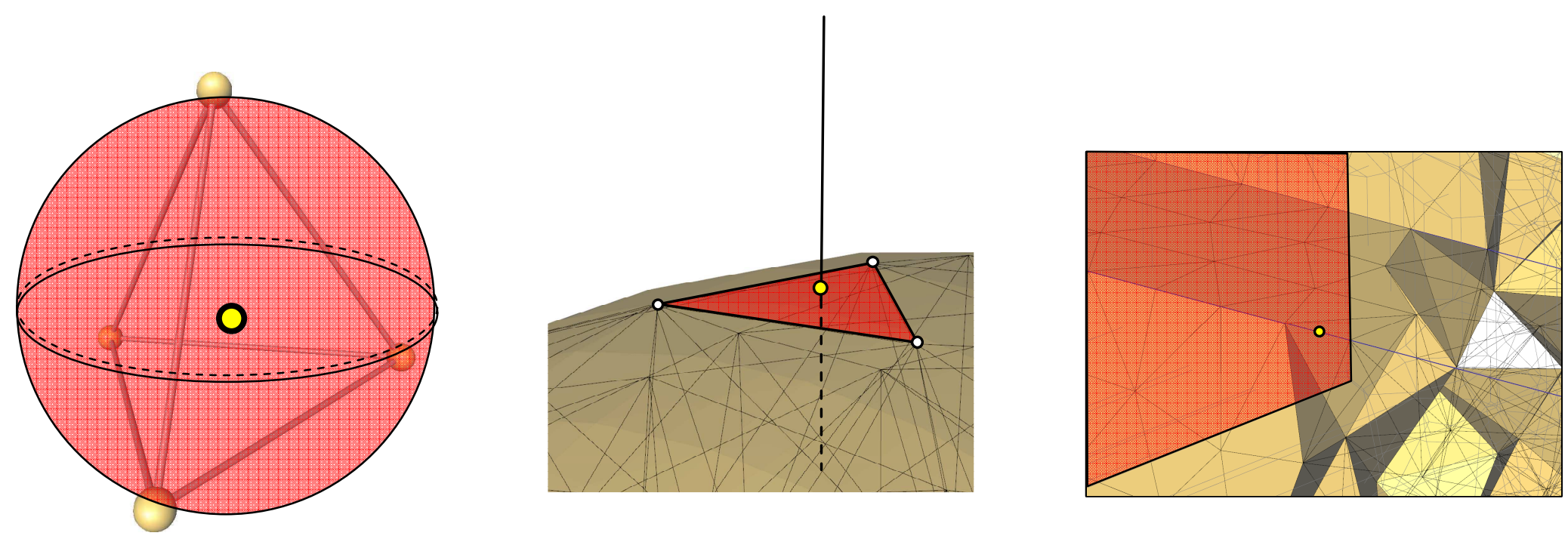


\section{Summary}




\section{Summary}

- From triangulation to quality meshes

- Mesh generation:

-2D: Preserves constraints exactly.
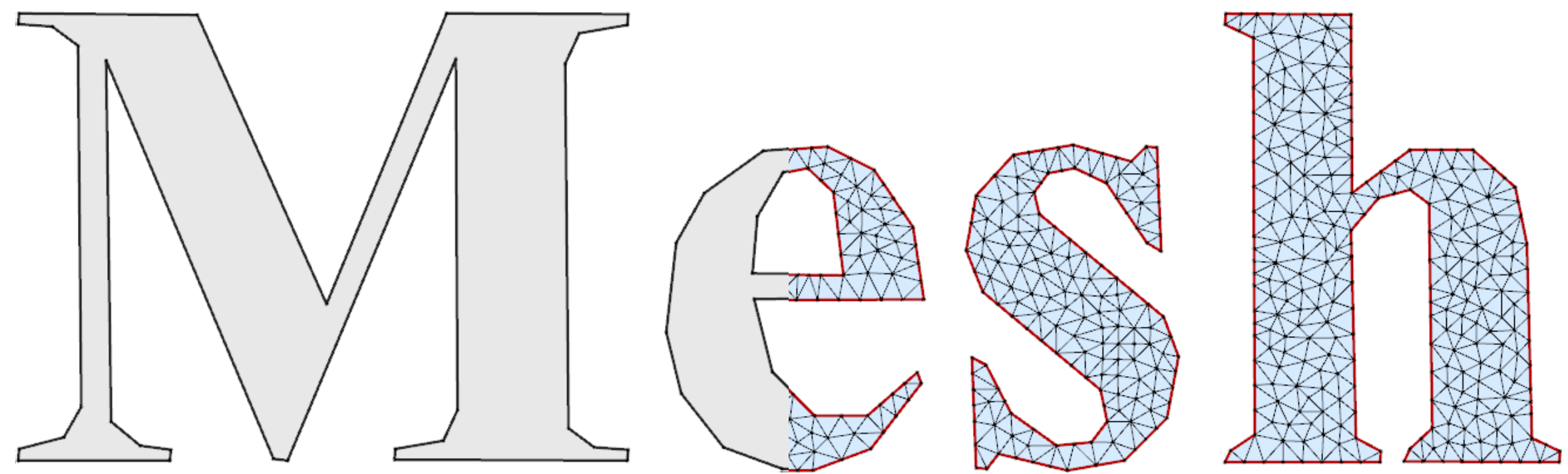


\section{Summary}

- From triangulation to quality meshes

- Mesh generation:

-2D: Preserves constraints exactly.

-3D: Interpolates boundary and sharp creases.

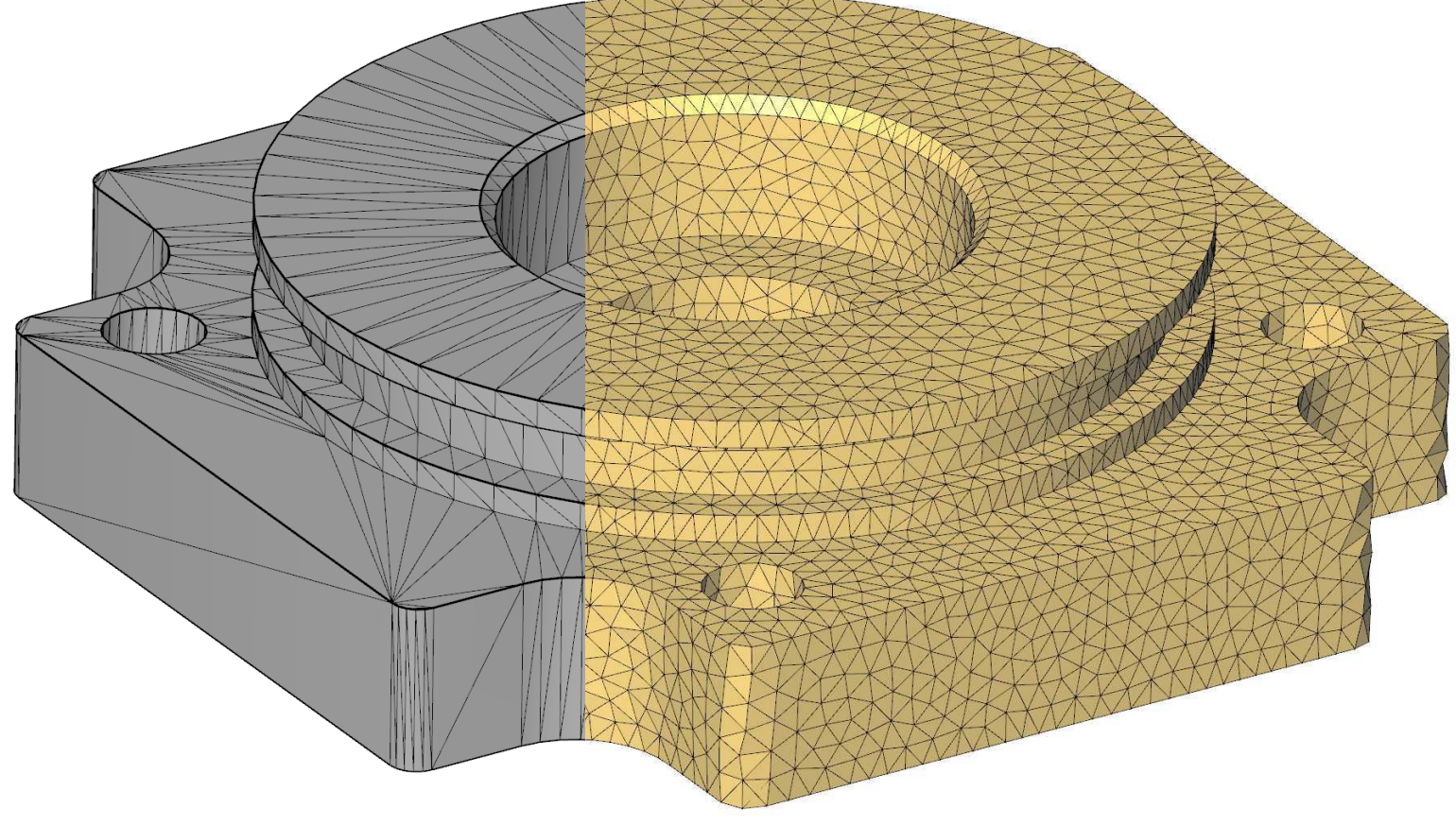




\section{Summary}

- From triangulation to quality meshes

- Mesh generation:

-2D: Preserves constraints exactly. $-3 \mathrm{D}$ :

- Interpolates boundary and sharp creases.

- Versatile through oracle-based design 


\section{See Also}

\section{DelPSC software}

\section{(based on CGAL)}

[Dey-Levine]

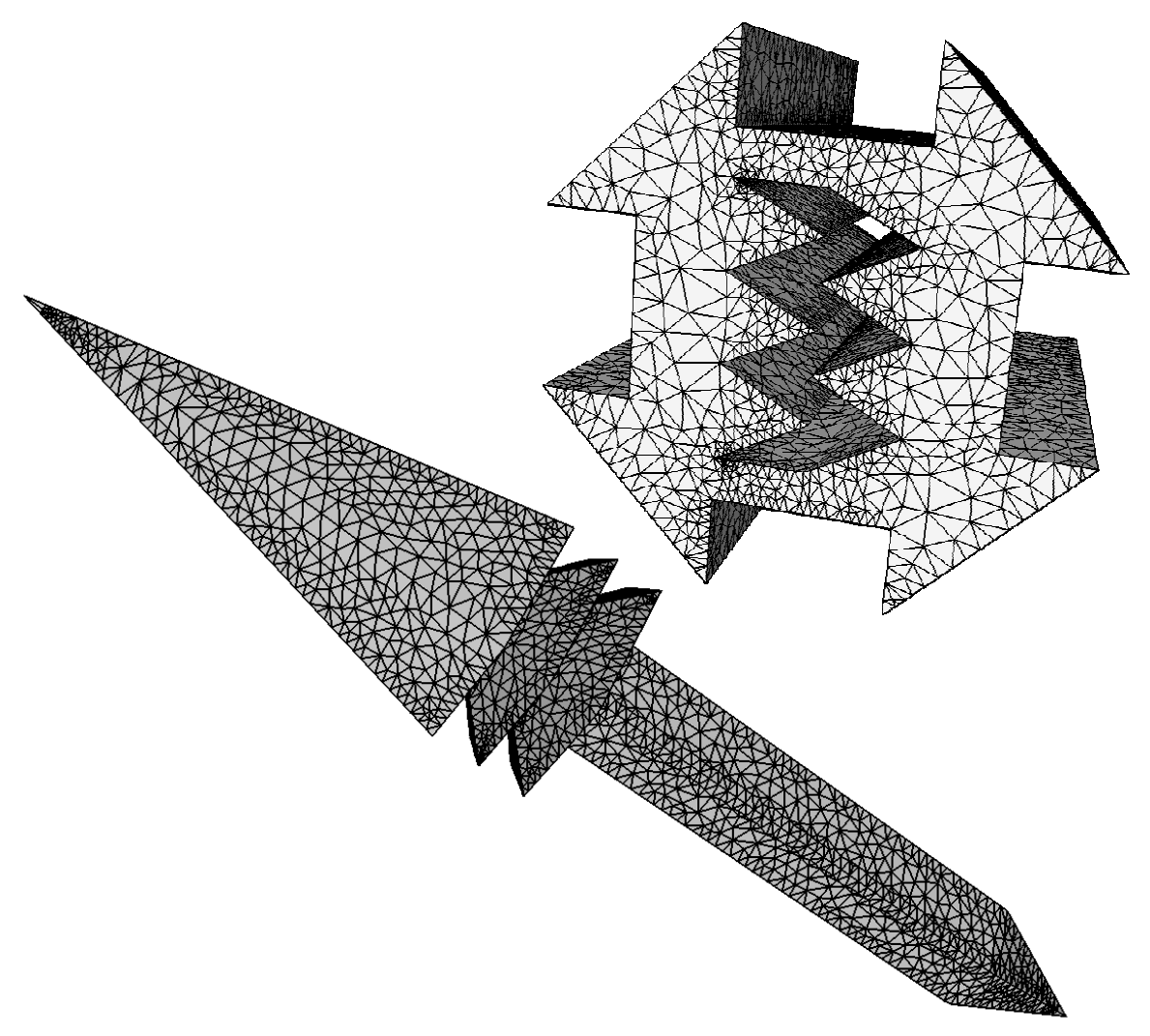

Skin surfaces
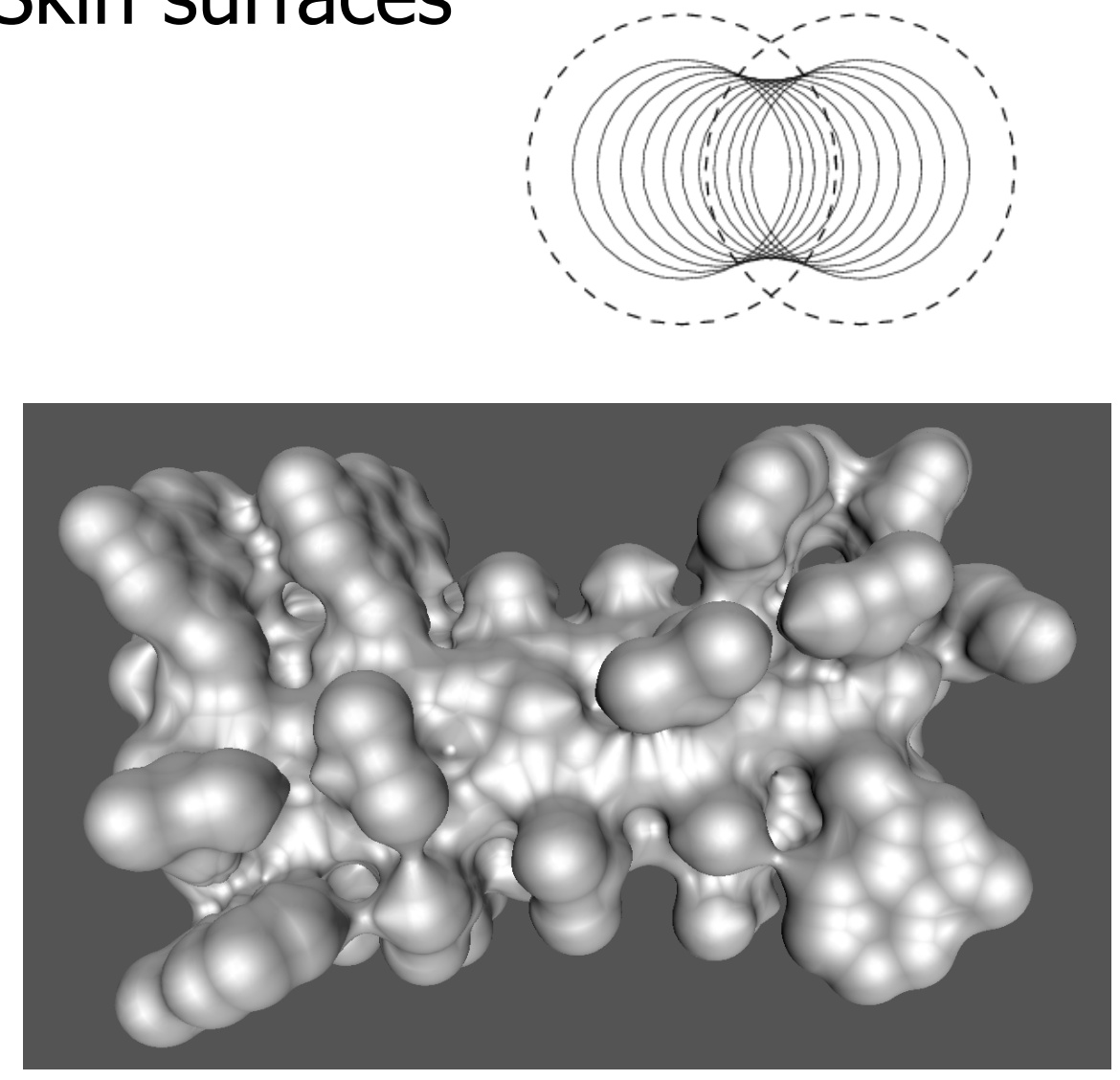

Online manual 